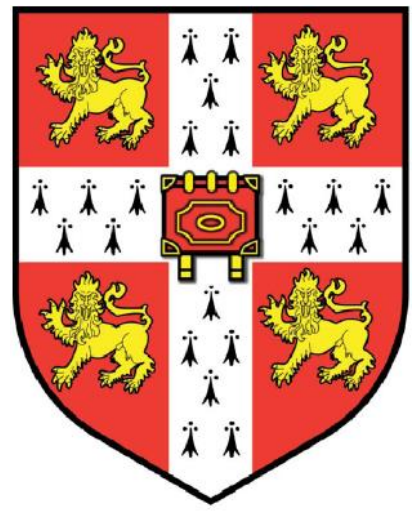

University of Cambridge

Department of Theoretical and Applied Linguistics

\title{
Implicit Learning of Semantic Preferences
}

\author{
Albertyna Paciorek \\ St John's College
}

A dissertation

submitted for the degree of

Doctor of Philosophy

September 2012 
To my beloved Mum - Ewa Zalewska-Paciorek,

Dad-Włodzimierz Paciorek,

Brother and best friend Adam Paciorek

and in loving memory of my Grandfather - Stanisław Zalewski 


\title{
Preface
}

I hereby declare that this dissertation is the result of my own work, and includes nothing which is the outcome of work done in collaboration except where specifically indicated in the text.

The material presented in Chapter 5, ERP Response to Semantic Implicit Learning, is the result of collaboration with Dr Nitin Williams. The signal analysis was performed by $\mathrm{Dr}$ Nitin Williams and the results are also included in his $\mathrm{PhD}$ thesis Single-trial analysis of Event-Related Potentials (ERPs), awarded by the University of Reading.

This dissertation does not exceed the regulation length of 80,000 words including footnotes, references and appendices but excluding the bibliography.

\author{
Albertyna Paciorek \\ University of Cambridge \\ 28 September 2012
}




\section{Acknowledgement}

I would like to express deep gratitude to my supervisor, Dr John Williams. I thank him for sharing his expert "super vision" as well as enthusiasm for implicit learning and psycholinguistics. If there were official gold medals for supervision he would certainly deserve one.

I would also like to thank other staff members of the Research Centre for English and Applied Linguistics (now part of the Department of Theoretical and Applied Linguistics), especially Dr Henriëtte Hendriks, Dr Anna Korhonen, Dr Brechtje Post and Dr Napoleon Katsos. Together with the administrative staff as well as my fellow PhD students and MPhil students they all contributed to creating a very supportive atmosphere in the department. I would like to extend particular thanks to Dr Napoleon Katsos for his friendship, encouragement and practical advice.

I significantly benefited from collaborating with a group of neuroscientists from the University of Reading: Dr Nitin Williams, Dr Slawomir Nasuto and Professor Douglas Saddy. I thank them for the fruitful discussions and their involvement in our crossdisciplinary project, the results of which are included in this dissertation.

Throughout the time I have enjoyed continuous support from my friends, for which I am deeply grateful, particularly to Magdalena Kubiak, TengTeng Xu, Fr Brian Creak, Sebastian Wydrowski, Guergui Nikiforov, Soraya Atmani, Chris Cummins, David Conlon, Martin Worthington, Misha Aizatulin, Noris Alsaadie, Bamned Sanitther, Jonathan Gilmour, Kornelia Korzec, Radosław Lach, Josephine Maes and Rafal Smietana. I owe special thanks to Hanno Rein and Marcin Dera for their help with JavaScript and preparing the online experiments, as well as Chris Cummins for proofreading the draft of this dissertation.

For the last four years I have been privileged to be a member of St John's College. I remain indebted to the Master and the fellows, especially Professor Patrick Boyde, for making St John's College such a marvellous community. I would also like to thank the College staff, including the team of porters and the buttery staff, especially Jorge Santana and Ania Olszanicka, for their friendliness, kindness and humour, which made a difference to every day.

None of this would have been possible without the loving support from my family. I am most thankful to my Mum, Ewa Zalewska-Paciorek, for her faith in me and unfailing encouragement at every step, my Dad, Włodzimierz Paciorek, for his great support, my brother, Adam Paciorek, who has been making my life better every day since 26 September 1988, and my beloved Grandfather, Stanisław Zalewski, who always believed in me and encouraged me to set the bar high. I also thank my Godmother, Bronisława Harpula, for her involvement and continuous care.

The four year financial support from the Arts and Humanities Research Council is gratefully acknowledged, as well as the contribution from St John's College and the University of Cambridge in my $10^{\text {th }}$ term. 


\section{Summary}

The research presented in this $\mathrm{PhD}$ dissertation examines the phenomenon of semantic implicit learning, using semantic preferences of novel verbs as a test case. Implicit learning refers to the phenomenon of learning without intending to learn or awareness that one is learning at all. Semantic preference (or selectional preference - as preferred in computational linguistics) is the tendency of a word to co-occur with words sharing similar semantic features. For example, 'drink' is typically followed by nouns denoting LIQUID, and the verb 'chase' is typically followed by ANIMATE nouns. The material presented here spans across disciplines. It examines a well-documented psychological phenomenon - implicit learning and applies it in the context of language acquisition, thereby providing insights into both fields.

The organisation of this dissertation groups its experiments by their methodology.

- Chapter 1 provides an overview of the current psychological and linguistic literature.

- Chapter 2 includes a pen-and-paper study carried out in a classroom environment on Polish learners of English, where awareness is assessed by subjective measures taken at each test question as well as a post-experiment questionnaire.

- Chapter 3 includes a collection of 5 computer-based experiments based on a falsememory paradigm. After exposure to sentential contexts containing novel verbs, participants are shown to endorse more previously unseen verb-noun pairings that follow the correct semantic preference patterns to the pairings that violate it. The result holds even when participants do not reveal any explicit knowledge of the patterns in the final debriefing. Awareness is additionally assessed using indirect measures examining correlations of confidence judgements with performance.

- Chapter 4 examines whether implicit learning of novel verb semantic preference patterns is automatic. To this end, a reaction time procedure is developed based on two consecutive decisions ("double decision priming"). The method reveals that semantic implicit learning, at least in the described cases, exerts its influence with a delay, in post-processing.

- Chapter 5 comprises research done in collaboration with Dr Nitin Williams, University of Reading. It documents an attempt at finding neural indices of implicit learning using a novel single-trial analysis of an electroencephalographic (EEG) signal, based on empirical mode decomposition (EMD) denoising.

- Chapter 6 presents a final discussion and indications for future research.

The main contribution of this dissertation to the general field of implicit learning research consists in its challenging the predominant view that implicit learning mainly relies on similarity of forms presented in training and test. The experiments presented here require participants to make generalisations at a higher, semantic level, which is largely independent of perceptual form. 
The contribution of this work to the field of Second Language Acquisition consists of empirical support for the currently popular but seldom tested assumptions held by advocates of communicative approaches to language teaching, namely that certain aspects of linguistic knowledge can develop without explicit instruction and explanation. At the same time, it challenges any view assuming that vocabulary learning necessarily relies on explicit mediation. The experiments collected here demonstrate that at least word usage in context can be learnt implicitly. A further contribution of this dissertation is its demonstration that the native language may play a key role in determining what is learnt in such situations.

A deeper understanding of the phenomenon of semantic implicit learning promises to shed light on the nature of word and grammar learning in general, which is crucial for an account of the processes involved in the development of a second language mental lexicon. 


\section{Contents}

Preface

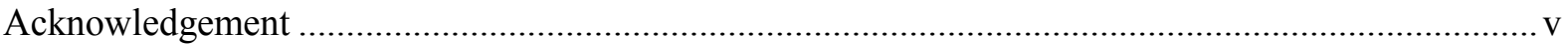

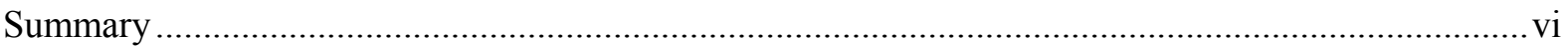

Chapter 1 The context of implicit learning research ............................................................... 1

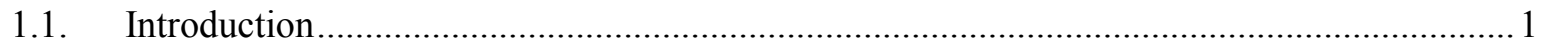

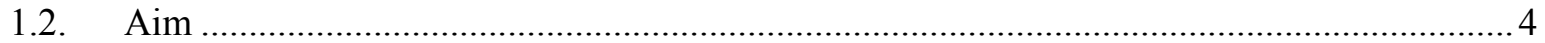

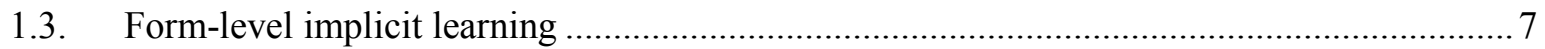

1.4. Form-level implicit learning in language acquisition ...................................................... 18

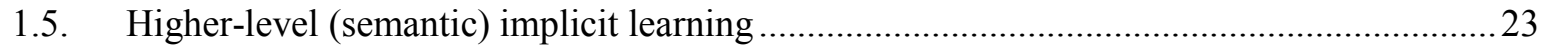

1.6. Higher-level (semantic) implicit learning in language acquisition ......................................2

1.7. Types, tokens, syntagmatic and paradigmatic learning - the case of semantic preferences. 31

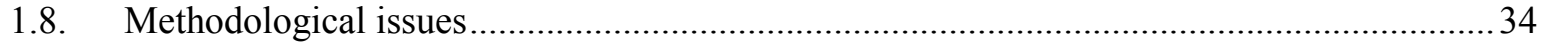

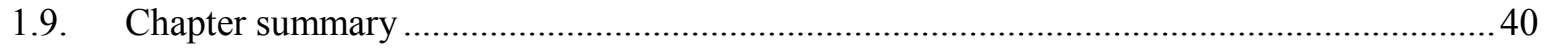

Chapter 2: Semantic implicit learning in acquisition of L2 English ..................................................42

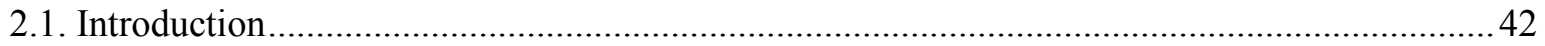

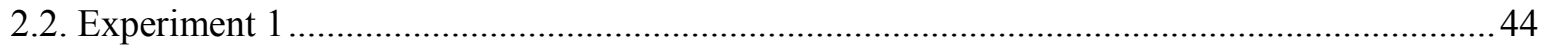

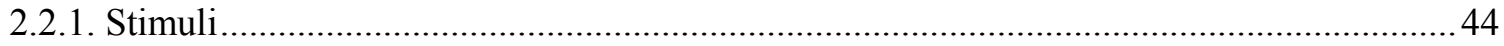

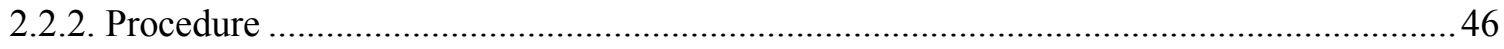

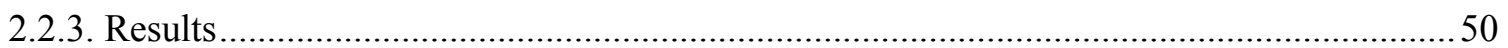

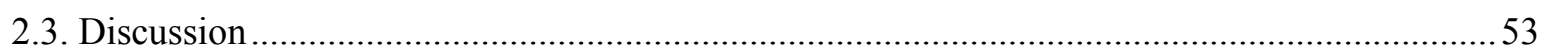

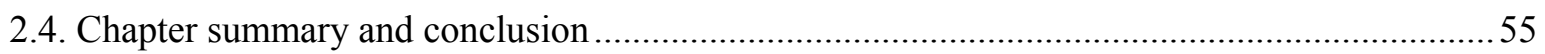

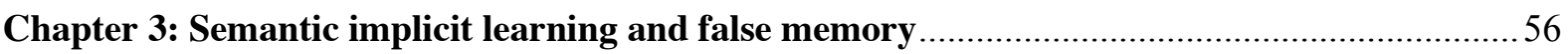

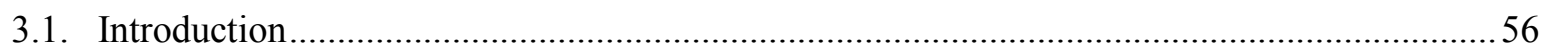

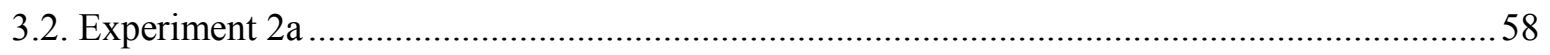

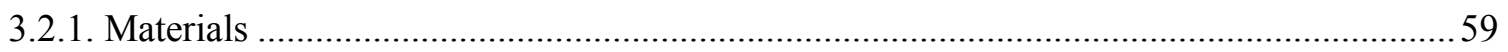

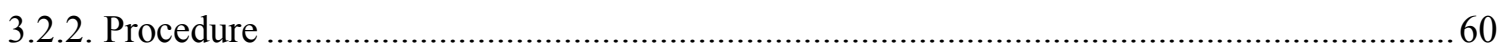

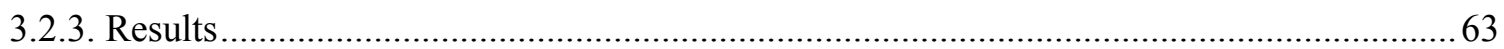

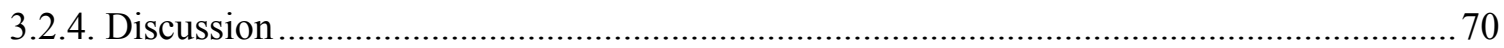

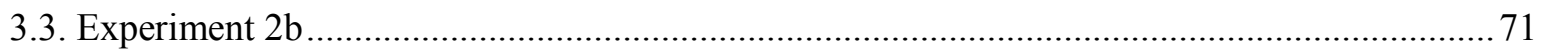




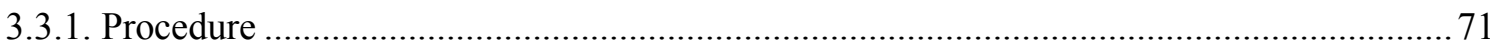

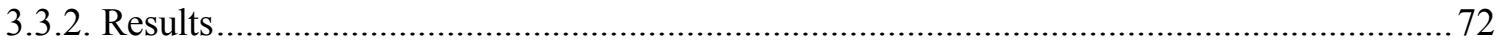

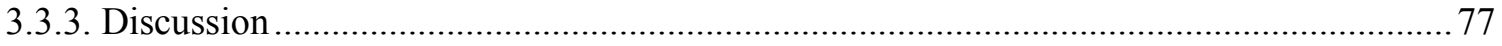

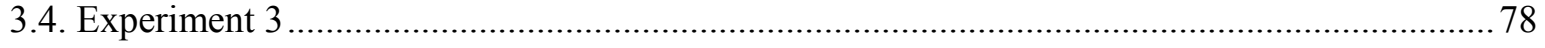

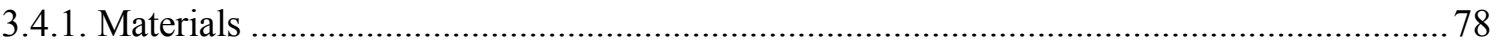

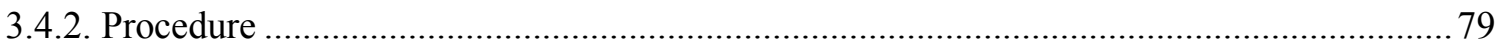

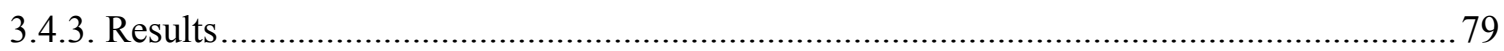

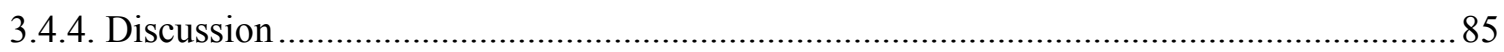

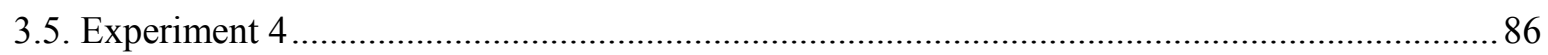

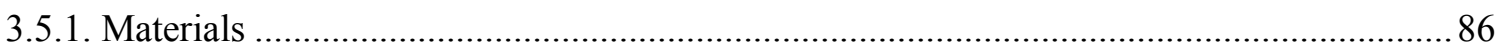

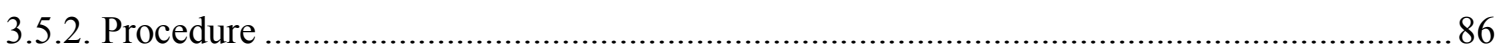

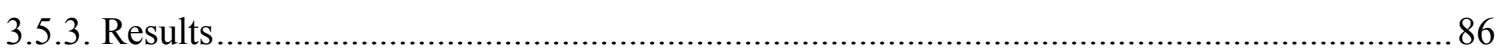

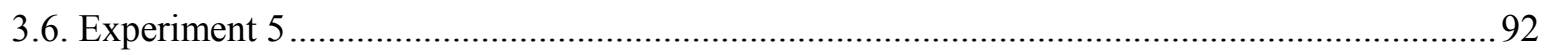

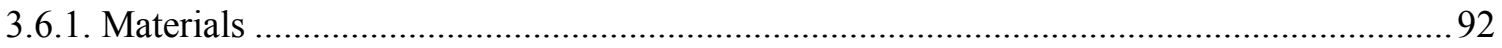

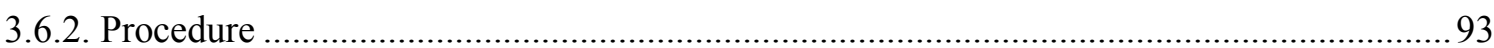

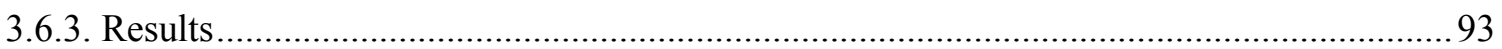

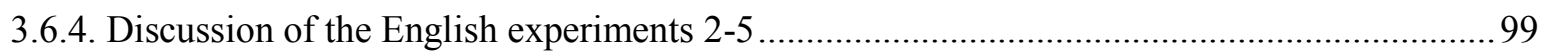

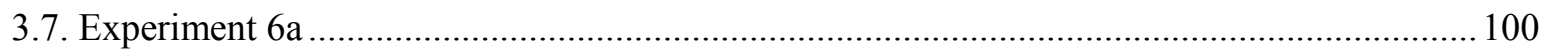

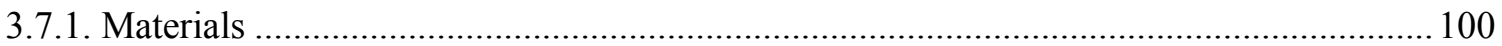

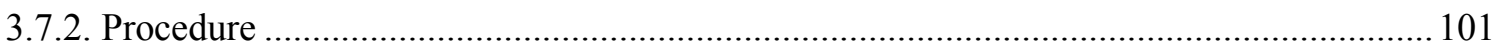

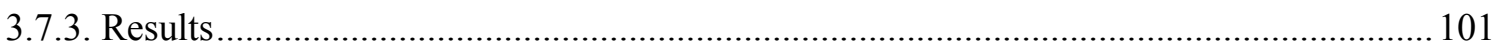

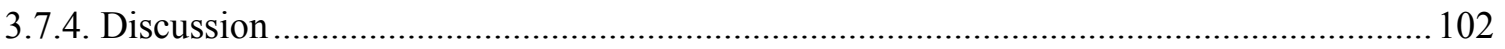

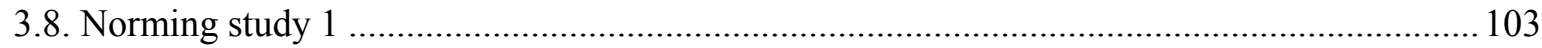

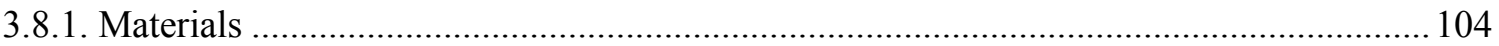

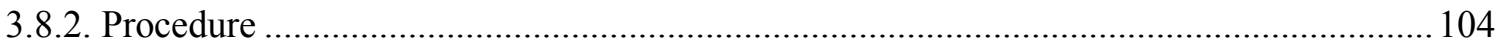

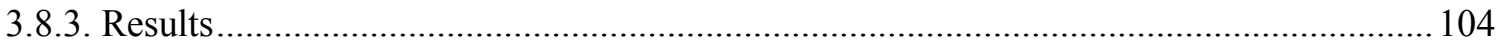

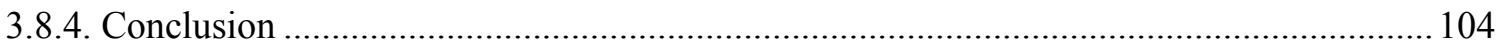

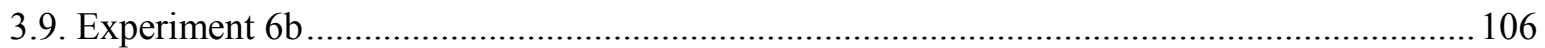

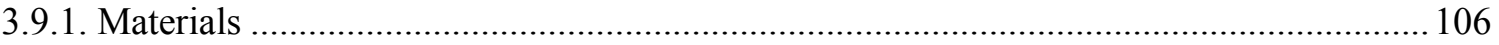

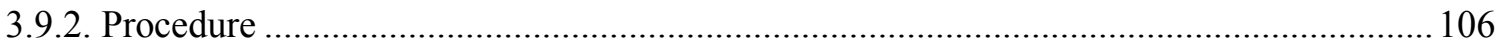

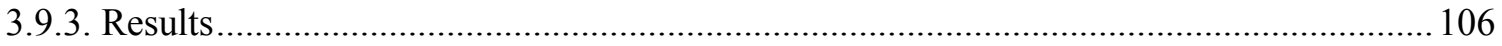

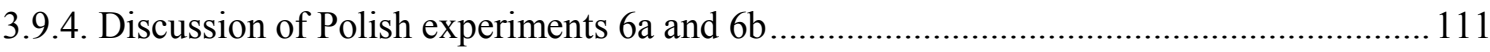

3.10. General discussion of false memory experiments.............................................................. 112

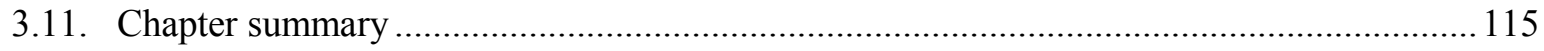




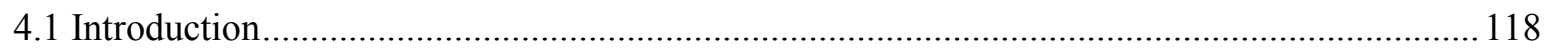

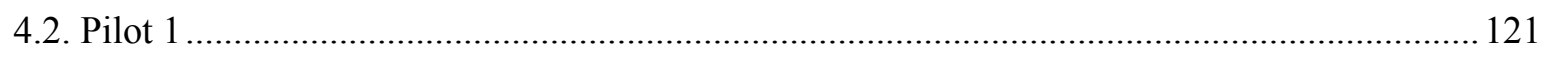

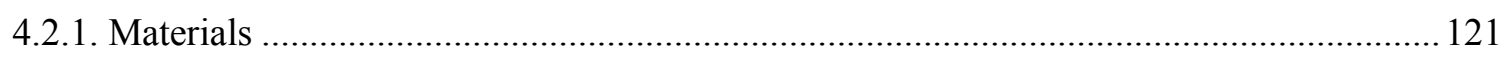

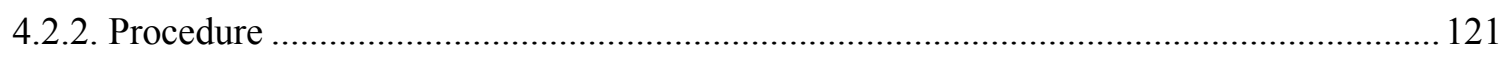

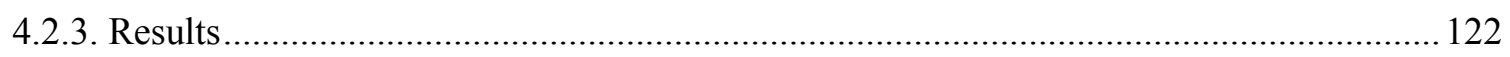

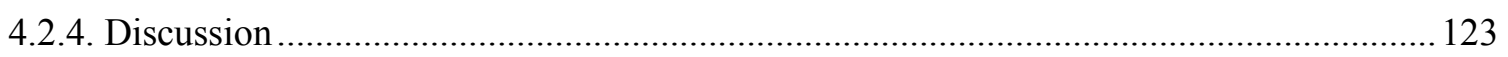

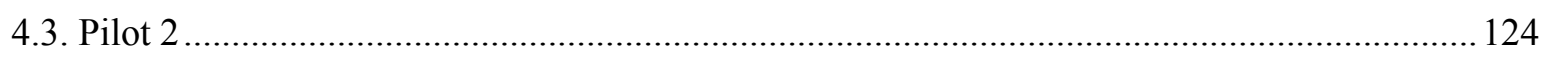

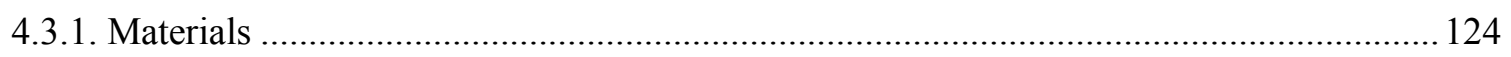

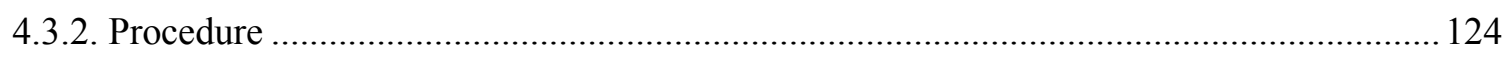

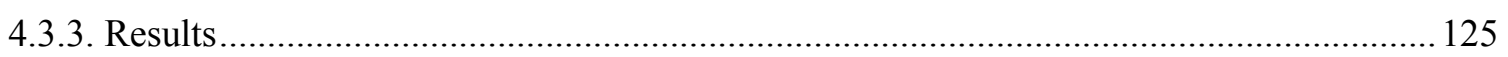

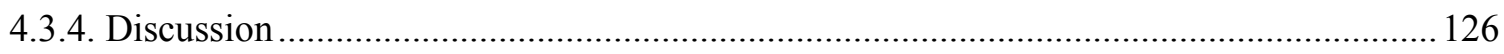

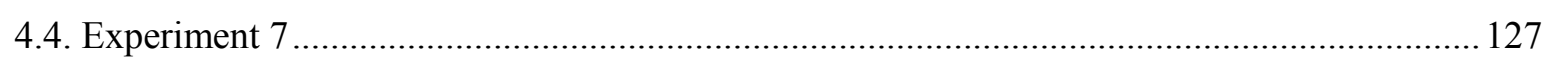

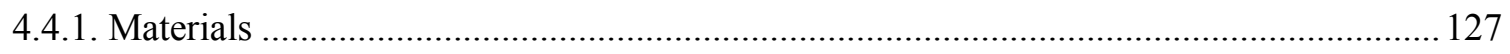

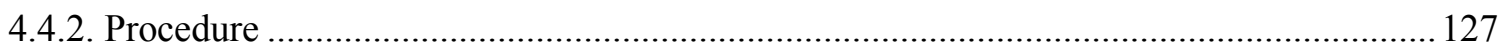

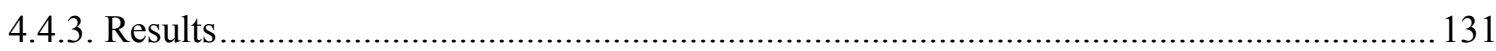

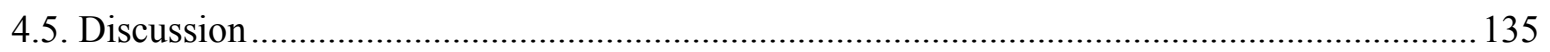

4.6. Pilot 3 - development of the "double decision priming" procedure ........................................... 135

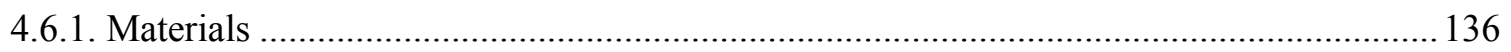

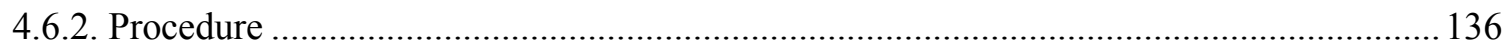

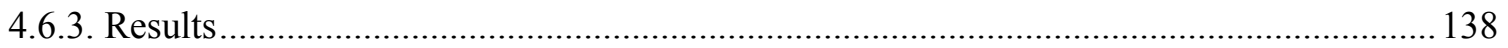

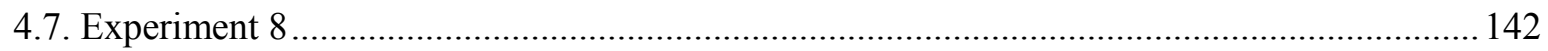

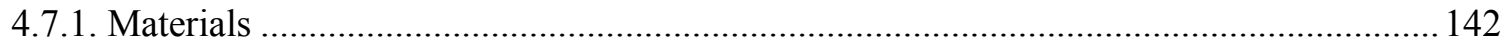

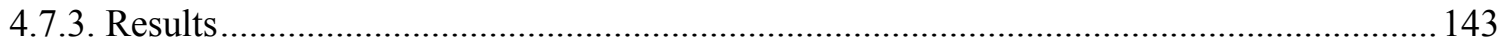

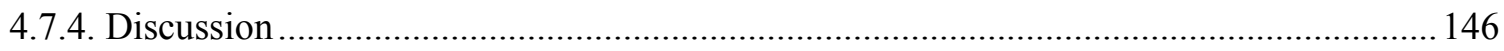

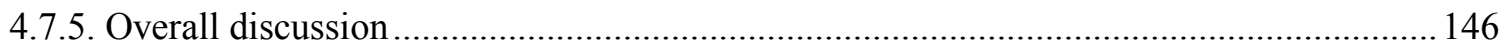

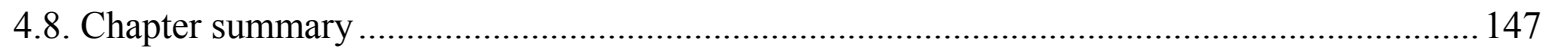

Chapter 5: Real-time ERP response to semantic implicit learning .......................................... 149

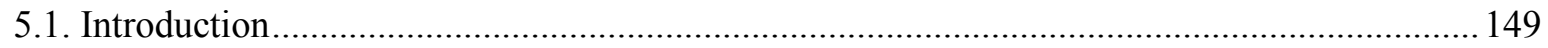

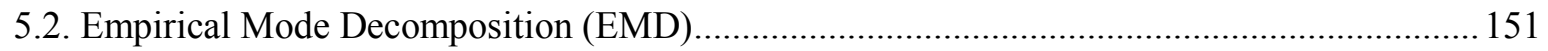

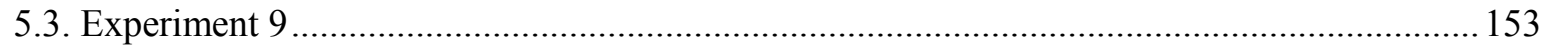

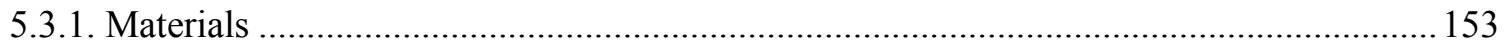

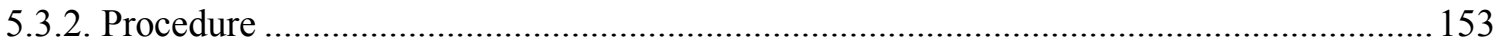

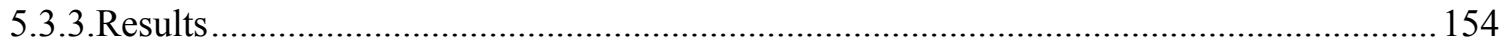

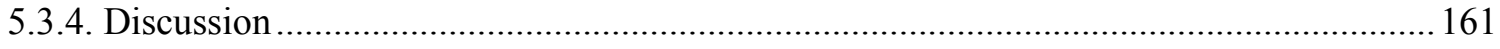




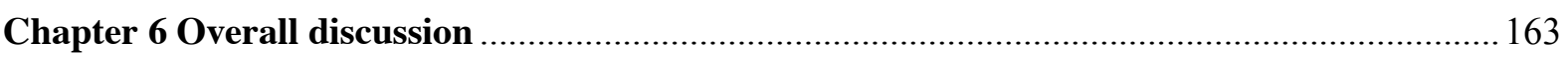

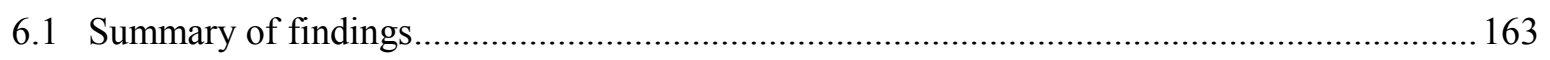

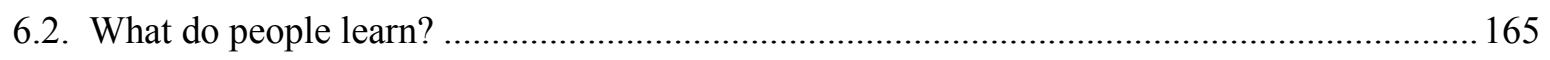

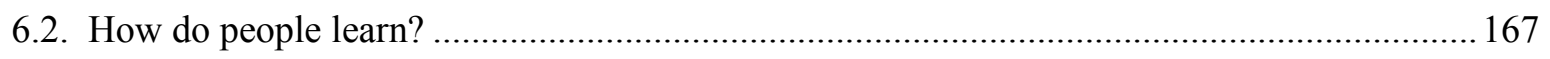

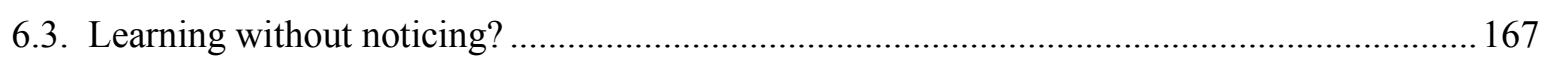

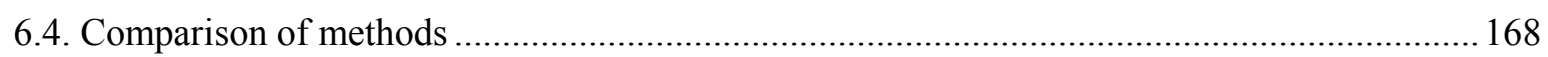

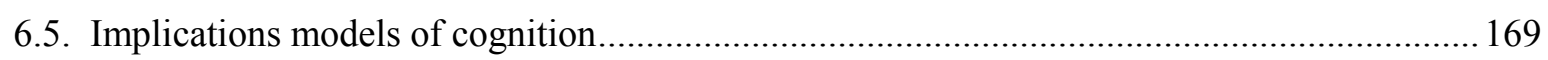

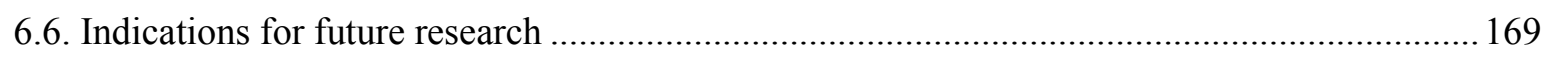

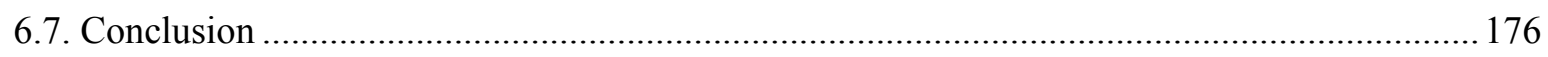

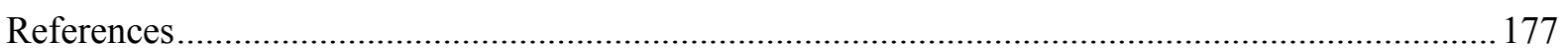

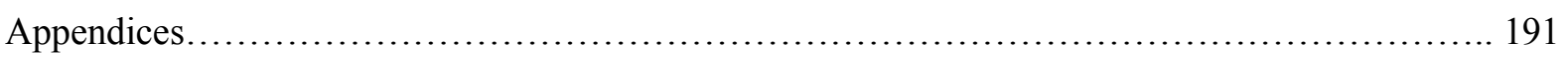




\section{Chapter 1 The context of implicit learning research}

\subsection{Introduction}

Knowledge of word meaning is to a large extent intuitive. Native speakers are confident they know what a given word means, yet may have difficulty explaining the finer aspects of its meaning. This becomes particularly apparent in the case of understanding near synonyms. While most English speakers agree that in (1) and (2) either big or large is acceptable, such a simple interchange is not always an option. For example in (3) large appears to be a much preferred alternative, whereas in (4) big generally sounds more natural.

(1) He entered into a room with shelves full of big/large leather-bound volumes.

(2) That shirt doesn't fit me. It's too big/large.

(3) DVDs are ideal for storing the large/*big amount of data associated with video.

(4) How big/*large are you around the waist?

The above examples illustrate implicit knowledge, the existence of which is rarely contested. It is the knowledge we are not aware that we have, yet which manifests itself in our behaviour in many areas of life, such as sports and games (e.g. knowing how to swim, ride a bicycle, play chess), music (e.g. dancing, playing an instrument), in creative processes (e.g. solving complex mathematical problems), in arts (e.g. having an intuitive sense of genuine art and fashion), even in social cognition (e.g. reading other people's intentions and mental states, 
understanding humour). A pinnacle example of implicit knowledge is the command of one's native language. People instantly notice sentences violating grammar, even without being able to state the underlying principles. Similarly, a wrongly played note will disturb even non-musicians, who cannot competently justify why. For some people, even cooking is a demonstration of highly impressive knowledge which unfortunately often remains incommunicable, despite efforts. Implicit knowledge is therefore the tacit, unverbalisable knowledge people may have, specific to a particular skill (Dienes \& Altmann, 1997; Cleeremans, Destrebecqz, \& Boyer, 1998; cf. Baars, 2002).

An issue which has sparked more controversy over the last few decades pertains to the question whether knowledge can also be acquired implicitly. If implicit knowledge exists, could implicit learning also exist? It is commonly defined as learning occurring without intention and without awareness of what is being learnt. As such, it contrasts with explicit learning, which refers to situations where people intend to learn something (possibly expecting a test) and engage their conscious knowledge in the process. While explicit learning typically involves forming and testing conscious hypotheses, implicit learning is believed to reflect absorption of statistical regularities in the environment, resulting in knowledge which is difficult to introspect.

Directly addressing these two types of learning bears particular importance for studies of the acquisition of both first and second languages. By popular assumption, the acquisition of the first language is an implicit process leading to implicit knowledge. To what extent a second language can also be learned implicitly, or in other words acquired, has been the subject of much investigation and rightfully deserves attention due to its important theoretical and practical implications, which will be discussed in this dissertation.

To date, numerous aspects of language have been shown to involve implicit or statistical learning ${ }^{1}$, including, among others, word segmentation (Onnis, Waterfall, \& Edelman, 2008; Saffran, Aslin, \& Newport, 1996), orthography (Pacton, Perruchet, Fayol, \& Cleeremans, 2011), phonotactics (Chambers, Onishi, \& Fisher, 2003), argument structure (Hudson Kam, 2009; Wonnacott, Newport, \& Tanenhaus, 2008; Wonnacott, Boyd, Thomson, \& Goldberg, 2012) and syntax (Chang, Dell, \& Bock, 2006; Rebuschat \& Williams, 2009). Even though not all material may be equally learnable by implicit means, interestingly, those

\footnotetext{
${ }^{1}$ It is assumed that implicit - or unconscious - learning operates as statistical learning. However, the converse is not true - not all studies demonstrating statistical learning address implicit learning (Perruchet \& Pacton, 2006).
} 
aspects of language which have proved challenging for learners, such as arbitrary gender categories (Braine et al., 1990; Brooks, Braine, Catalano, Brody, \& Sudhalter, 1993; Guillelmon \& Grosjean, 2001; Williams, 2003), or scrambling (Williams \& Kuribara, 2008; Iwasaki, 2003), also tend to be the ones with which second language learners notoriously experience difficulties. Furthermore, individual differences in implicit learning have been shown to correlate with language abilities independently of other factors such as IQ or working memory (Conway \& Pisoni, 2008; Conway et al., 2009; Misyak, Christiansen, \& Tomblin, 2010; Misyak, Goldstein, \& Christiansen, in press). Even language disabilities have been associated with impairments of particular types of implicit learning, for example dyslexia with impaired implicit sequence learning (Howard, Howard, Japikse, \& Eden, 2006; Jimenez-Fernandez, Gracia Vaquero, Jimenez, \& Defior, 2011), and specific language impairment and agrammatism with a deficiency in artificial grammar learning (Evans, Saffran, \& Robe-Torres, 2009; Plante, Gomez, \& Gerken, 2002; Christiansen, Louise Kelly, Shillcock, \& Greenfield, 2010). Together these results point to the interrelation between language and statistical implicit learning mechanisms.

In psychology, the research on implicit and statistical learning has so far focused on surface-form regularities. As a result, in language acquisition as well as psychology, much is now understood about the crucial importance of such factors as form frequency or salience in the input. The question which occupies much of the present day psycholinguistic investigations is: could all of language learning be modelled only by tracking surface form regularities? On the one hand, statistical models are capable of demonstrating some mechanisms of generalisation to new stimuli, which could be compared to generalised linguistic knowledge. On the other, inherent in language is the fact that words and structures carry meaning. Still relatively little is known about whether and to what extent meaning may contribute to the formation of linguistic knowledge. Research on whether and how people learn meaningful regularities automatically and without conscious control (implicitly) may be necessary in order to resolve this question.

This relative lack of empirical evidence contrasts with the assumption put forward in numerous pedagogical approaches as well as theoretical accounts, especially the functionalist and usage-based ones, which often take meaning as a starting point for the formation of linguistic constructions or even syntactic categories. Even though many aspects of word meaning, such as referential meaning (eg. 'cat' picks out CATS in the world), are assumed to be explicit, or declarative, nevertheless the learning which subsequently occurs, after the 
word forms have been mapped to the relevant semantic representations, is predominantly implicit - although little is known about its scope. For example, Ellis (2005) proposes that fine-tuning or tallying of representations does take place without conscious involvement and that "the results of this tallying provide an optimal solution to the problem space of formfunction mappings and their contextualized use" (Ellis, 2005, p.307).

The proposition that implicit learning may be involved in the formation of formfunction mappings and their contextualized use is not as straightforward as it may seem. To date, the development of these aspects of linguistic knowledge has seldom been empirically addressed within an implicit learning framework. Indeed, such attempts have only been made in the last decade, and then not without controversy (Williams, 2005; Hama \& Leow, 2010).

\subsection{Aim}

Is there or can there be, unconscious induction and abstraction? This is the heart of the acquisition versus learning distinction in the foreign language field and the heart of the controversy over implicit learning in psychology.

(Schmidt, 1995, p. 31)

The aim of this dissertation is to empirically examine whether implicit learning processes are involved in enabling adult learners to form semantic generalisations from linguistic input. It focuses therefore on semantic implicit learning, which can be defined as a specific type of implicit learning - one which operates on regularities involving meaning, as opposed to just perceptual forms. The experiments reported here address the development of implicit knowledge of word use in appropriate semantic contexts. Well-formedness of utterances, after all, does not only require satisfaction of syntactic constraints but of semantic ones as well. Do adult learners learn to satisfy these semantic constraints (or preferences) incidentally and without awareness?

By addressing this question, the aim is to contribute to one of the major debates in modern cognitive psychology, namely: does learning without awareness exist and if so, what is and what is not learnable that way? Opinions are divided, and there is a fair amount of scepticism whether it is possible to learn even the simplest associations implicitly. For 
example, Lovibond \& Shanks (2002) argued that even simple Pavlovian conditioning relies on conscious predictive relationships between events. In psychology, the putative demonstrations of implicit learning so far have predominantly been based on perceptual form-level regularities, e.g. letter sequences. This has led to the conclusion that the implicit learning mechanism relies on unconscious counting, and the reported learning effects reflect sensitivity to form frequencies (Ellis, 2002). However, such demonstrations could be regarded as involving chunking, which could rely, at least in part, on conscious recognition (Perruchet \& Vinter, 1998). Of crucial importance, therefore, is the adequate measurement of awareness. Current methods have often been susceptible to the criticism of not being sensitive enough (Shanks \& St. John, 1994). And if implicit learning is viewed as a type of statistical learning and form-counting, even among those who do advocate its existence there remains much doubt whether it could lead to unconscious generalisation or abstraction, in other words whether it could lead to knowledge which is not dependent on the experienced perceptual forms (see Section 1.3).

In the field of second language acquisition research scepticism about the possibility of implicit learning is perhaps most clearly expressed in the work of Schmidt (e.g., Schmidt, 1990; 1995), and resonates in his seminal "noticing" hypothesis. Schmidt was primarily concerned with the roles that attention and awareness play in the learning process. He proposed "noticing" to be the minimal combination of attention and a low level awareness, necessary and sufficient for converting input to intake (Schmidt, 1990). He proposed that stimuli outside focal attention and therefore outside awareness might activate preexisting memory representations, but only subliminally, and that subliminal learning is impossible.

Once 'noticed', a given aspect of language may be consciously analysed, compared to other things which were 'noticed', giving rise to a higher level of awareness 'understanding' (which involves what is generally understood as 'thinking'). Schmidt wrote sceptically about the possibility of forming generalisations and abstract rules without noticing at the level of understanding. But, could it be it possible? This is the question which the research contained in this dissertation addresses. Schmidt himself identified "unconscious induction and abstractness of the knowledge that results from learning" as "the most important issues involved in implicit learning studies" (Schmidt, 1995, p.35).

Working from a more pedagogical perspective, Krashen (1981) first proposed the distinction between unconscious acquisition and conscious learning. While certain aspects of language may be naturally acquired, others require conscious learning. Identifying exactly 
which ones can be acquired naturally is the Holy Grail for language pedagogues. In terms of lexical learning, Paradis, (2004) proposed a distinction between 'vocabulary' and 'lexicon'. With vocabulary encompassing referential meaning ('cat' picks out CAT in the world) requiring explicit processes (Ellis, 1994), other aspects of word-knowledge, such as collocational behaviour, belonging to the lexicon, may be implicit.

Here implicit learning is investigated with respect to the acquisition of words' semantic (selectional) preferences. The term dates back to Sinclair (1996). Semantic preferences are tendencies of words to co-occur with words of certain semantic classes in a given role, e.g., the verb chase is followed by ANIMATE nouns as direct object, or drink by nouns denoting LIQUID. Very often, as in cases like these, semantic preferences are logically determined by word meaning. They may, however, be arbitrary as well, such as the big/large examples (1)-(4) in the section above. The Longman Dictionary of Contemporary English (2001) gives a helpful elucidation of the difference between the two near-synonyms, mentioning that big is more often used to describe people than large, while large is used with quantity words such as amount, scale, number, quantity, extent, proportion, percentage, part, volume, and area. Indeed, the first sentence of this dissertation provides an example: "knowledge of word meaning is to a large extent intuitive".

An important role for semantic preferences in meaning disambiguation has been identified in the field of machine learning and computational linguistics (e.g. John \& McCarthy, 2000; Resnick, 1997). In natural language linguistics, so far, considerably less attention has been paid to them.

Yet semantic generalisations are ubiquitous in language. They not only concern predicates, but can also be found in certain noun collocations. For example, in English it is correct to say 'a pack of dogs/hounds/wolves/coyotes/mules/rats/weasels' and 'a swarm of bees/insects/mosquitoes/bats/ladybirds'. It sounds unnatural and even humorous to say *a pack of bats, *a pack of insects or *a swarm of dogs. It is therefore plausible that in the process of learning, the speaker's knowledge of the meanings of these words gains the shape of, roughly: SWARM + [ANIMAL, FLYING, rather SMALL, most likely an INSECT], PACK + [ANIMAL, FOUR-LEGGED, most probably DOG-LIKE]. Native speakers develop such generalised notions incidentally, and possibly are not always aware of what exactly categorises legitimate collocates. Second language learners may need to be explicitly taught about such regularities. However, the ubiquity of similar phenomena in language precludes the possibility of all of them being successfully taught that way. It therefore makes sense to assume that learners also 
develop much of their knowledge about semantic preferences and semantically restricted collocations incidentally. Could this process also be implicit and lead to knowledge of which learners are not aware, similarly to native speakers?

To date, conclusions from the mainstream implicit learning research cast doubt on the possibility that it could lead to the development of abstract generalisations going beyond the training material (e.g. Conway \& Christiansen, 2006). On the other hand, semantic preferences are abstract as they reflect knowledge of conceptual categories rather than specific exemplars or perceptual surface features of the experienced exemplars (words). Should semantic implicit learning be demonstrated, how could it be reconciled with the current accounts of the implicit learning mechanism? The following section contains a summary of implicit learning research in experimental psychology and an overview of different paradigms used. It will be suggested that the perceived limitations on generalisation/abstraction could be the result of the fact that most research so far has focused on regularities only pertaining to surface forms, which do not lend themselves so naturally to generalisation/abstraction as do words in natural language.

\subsection{Form-level implicit learning}

The majority of implicit learning demonstrations to date have addressed learning perceptual surface-form regularities. Experimental stimuli usually involve strings of letters, sounds or patterns on a visual display, which are often generated by complex rule systems. Arthur Reber is considered the pioneer in this area (Reber, 1967, 1989 and other). His first evidence for learning without awareness involved participants developing unconscious knowledge of regularities in letter strings, which, unbeknown to them, were generated by an underlying system, such as the one presented in Figure 1.1. Such systems were known as finite-state grammars as, just like natural language grammars, they can generate an infinite number of well-formed strings with a finite set of rules. Later the term artificial grammar was coined. Historically, the idea was inspired by the project Grammarama, started in 1957 by George Miller, which aimed to examine the learnability of formal linguistic systems. Although the extent to which artificial grammars reflect the actual structure underlying natural language may be debated, investigating the nature of the knowledge gained through learning such 
sequences of letters or symbols may offer a first step towards understanding the nature of the implicit learning mechanism, in other words, the mechanism of acquisition.

Typical artificial grammar learning (AGL) experiments consist of two parts. First, participants are exposed to letter strings, such as PVPXVPS, TSSXXVPS, TSXS and told to memorise them as part of an experiment on rote memory. They are not told that the strings are generated by an underlying system of rules. Afterwards, they are presented with new strings, informed that there was a system and asked to indicate which of the strings conform to it, or are, in other words, grammatical. Participants' overall performance is typically above chance, even though they claim not to have any idea of the underlying grammar. The results have been replicated even with improved methods for probing conscious knowledge, such as asking participants to provide subjective judgements of awareness (e.g. whether they are responding based on a guess, intuition, rule or memory for a fragment) and a confidence rating for each response. Participants still discriminate the correct and incorrect strings above chance when they claim to be purely guessing and when higher confidence does not correlate with better accuracy (Dienes \& Scott, 2005). Particularly impressively, similar learning effects have been reported among amnesic patients (e.g., Knowlton, Ramus, \& Squire, 1992), which adds weight to the theory that the knowledge in artificial grammar experiments develops without conscious mediation.

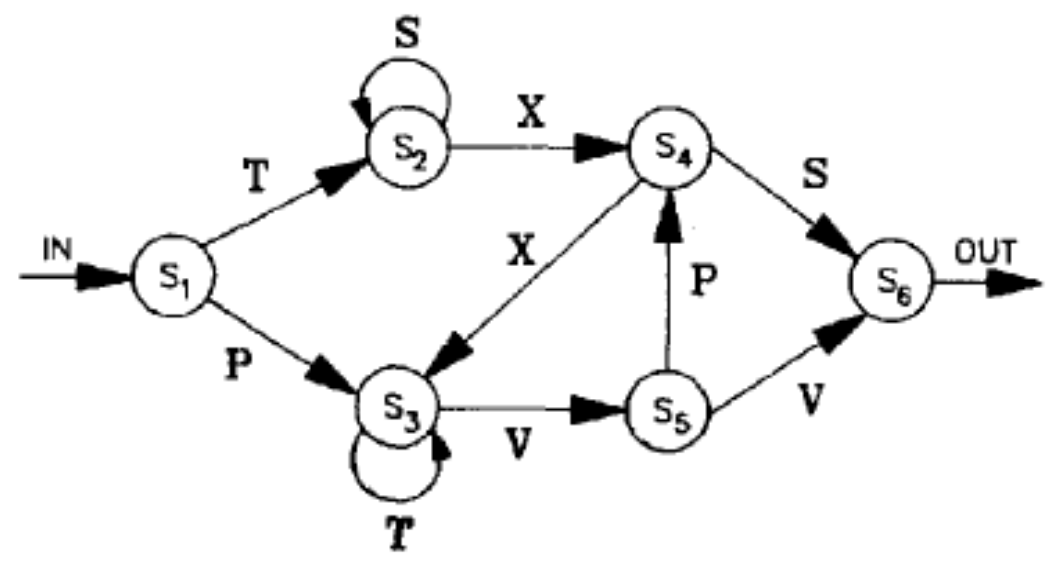

Figure 1.1. Schematic diagram of an artificial grammar, from Reber (1989). Stimuli are generated by following any path of arrows leading from the initial State 1 to the terminal State 6.

The knowledge gained in such tasks has been shown to some extent to transfer to new stimuli. The issue has been examined by changing the letter set between training and test, e.g. PVPXVPS in training would correspond to the string AFAHFAC in test. The resulting strings 
are thus compliant with the same grammar, but involve different surface forms. Typically, people's grammaticality judgements for the changed letter sets remain above chance (Reber, 1967; Reber, 1969; Knowlton \& Squire, 1996; Mathews et al., 1989), although the overall accuracy is lower than for the same letter sets (the so called 'transfer decrement effect'). Interestingly, transfer has even been shown across modalities, as for example when colours presented in training are changed to colour names at test, or even more impressively, when the grammar used to generate letter sequences in training is used to generate tone sequences at test or vice versa (Altmann, Dienes, \& Goode, 1995; Manza \& Reber, 1997; Gomez, Gerken, \& Schvaneveldt, 2000; Knowlton \& Squire, 1996; Tunney \& Altmann, 2001; Tunney \& Shanks, 2003), indicating that at least to some extent the knowledge gained in artificial grammar tasks is abstract - understood as generalisable to new stimuli.

In a different paradigm relying on surface-form regularities, Lewicki and colleagues (Lewicki, Czyzewska, \& Hoffman, 1987; Lewicki, Hill, \& Bizot, 1988) demonstrated implicit learning of visual sequences. In a four-choice serial reaction time task (SRT), participants were asked to track the targets appearing in one of four quadrants on a computer screen by pressing the corresponding button as quickly as possible. The sequences were generated with highly complex rules. For example, in Lewicki et al. (1987) the stimuli were presented in blocks of seven trials. Unbeknown to the participants there was a regular, highly complex and non-salient relationship between the position of the target on the $7^{\text {th }}$ trial and the quadrants in which it was presented on the $1^{\text {st }}, 3^{\text {rd }}$, and $6^{\text {th }}$ trials of that block, while the positions on the $2^{\text {nd }}$ and $5^{\text {th }}$ trials were random. In Lewicki et al. (1988) blocks contained five trials each; the target locations in the first two were random, while the last three were determined by the rules. Participants' performance on the final trials of each block, the locations of which were predictable from the rules, was faster and more accurate than on the random trials, despite the fact that they expressed no awareness of any rules. Interestingly though, unlike in artificial grammar learning, transfer to stimuli with dissimilar surface features typically fails in SRT tasks (Stadler, 1989; Willingham, Nissen, \& Bullemer, 1989; Perruchet, 1994).

Another line of research has addressed unconscious learning of less complex regularities. In 'invariant learning' tasks, initiated by McGeorge \& Burton (1990), participants' behaviour is biased according to whether or not a particular feature is present in a given stimulus. In the original study participants initially performed an arithmetic task on a set of four-digit numbers. They were not told that each of the numbers in the training phase 
contained the digit ' 3 ' (the 'invariant'). In the subsequent, unexpected forced choice task participants saw pairs of four-digit numbers and were asked to indicate which one in each pair appeared in the previous phase. In fact, all the numbers were new, but half of them contained a ' 3 ', while the other half did not. Participants were more likely to choose the numbers containing ' 3 ', even if they had no awareness of the invariant and expressed surprise when informed about it.

In all the above demonstrations, in AGL, SRT and invariant learning tasks, implicit learning was shown to influence people's decisions. There is evidence that knowledge acquired through implicit learning, at least in the context of visual covariation, can exert even further top-down effects in the form of attentional guidance: in other words, it can constrain what people expect and where they direct their attention. For example, in Chun and Jiang's (1998; 1999) "contextual cuing" paradigm participants perform visual search for a target object, such as a rotated ' $T$ ', in an array of rotated ' $L$ ' distractor shapes (see Figure 1.2a). Unbeknown to them, displays are repeated such that certain configurations of distractors (invariant contexts) are always paired with specific target positions so that visual context predicts target locations. If participants are sensitive to contextual information while performing the search task, then their performance for old displays should get faster over time, relative to trials where the distractor positions are determined randomly (baseline). This is indeed what is found (Figure 1.2b). Therefore visual context does serve to guide (or cue) attention to the target, facilitating search. Crucially, in a subsequent recognition task participants are unable to distinguish the repeated arrays from random ones, suggesting the effect is implicit. In daily life, such sensitivity to statistical regularities in the environment facilitates effective interaction with the visual world in general (e.g. Brockmole, Castelhano, \& Henderson, 2006).

Similar visual orienting effects were reported in Lambert, Naikar, McLachlan, \& Aitken (1999) and Lambert (2003). Participants saw pre-trial cues of two letters - 'W' on the left and ' $\mathrm{S}$ ' on the right, and in randomly chosen trials with ' $\mathrm{S}$ ' on the left and ' $\mathrm{W}$ ' on the right. What they did not know was that the display was programmed so that the target usually appeared on the same side as one of the letters (' $\mathrm{W}$ ' for half of the participants and ' $\mathrm{S}$ ' for the other half). Again, the authors found evidence for participants' attention being oriented by the predictive cues (and called this phenomenon "derived attention"). 
(a)

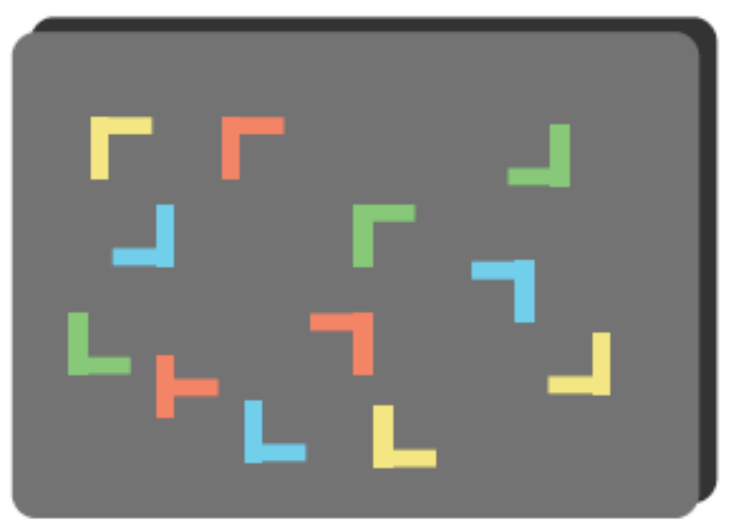

(b)

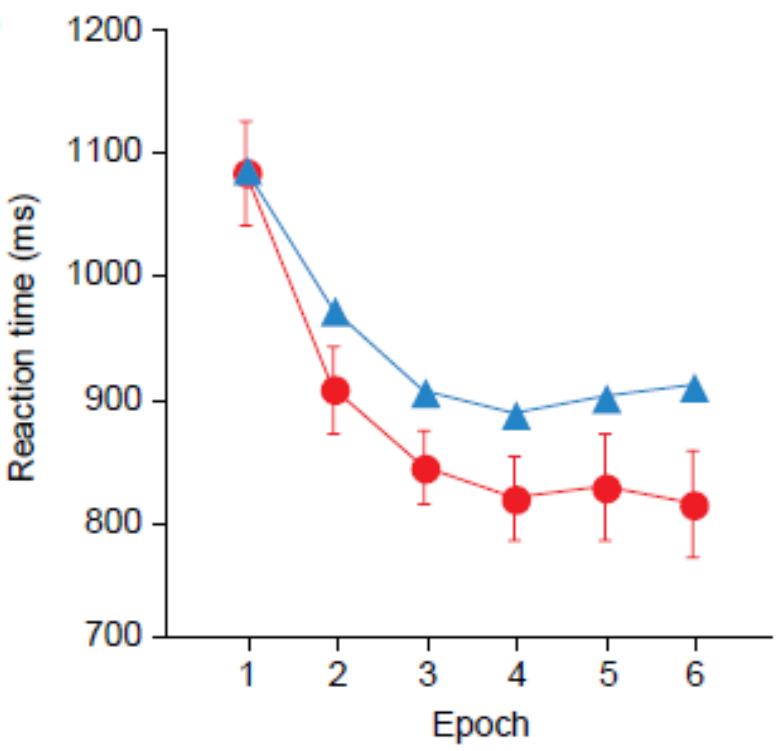

Figure 1.2. Spatial contextual cueing, (a) a sample search array, (b) search performances as a function of epoch (groups of five blocks, each with 12 trials for each condition) faster for old configurations than new configurations. Data from Chun \& Jiang (1998), image from Chun, 2000).

Overall, the above presented experiments illustrate a sample of thoroughly different procedures which have been developed to address the phenomenon of implicit learning. They represent the mainstream strand of implicit learning research, which focuses on regularities occurring at the surface level, usually perceived visually. What participants learn, however, is clearly not tied wholly to the perceptual, surface features, because that would preclude the effect of transfer. For example, transfer between such equivalent concepts as digits and words for digits, colours and colour names, etc. indicates that the learning mechanism must, at least to some extent, operate on a conceptual rather than a perceptual level. But what about transfer across modalities, e.g. between letters and tones? Initially, the solution put forward in all of the above presented cases (apart from contextual cueing) was that participants indeed absorb complex rules. However, the fact that performance in transfer conditions is always lower than in non-transfer indicates that even if participants did absorb some rules, they would not correspond exactly to the ones used to generate the stimuli. Crucially, though, any potentially learned rules are clearly not consciously applied, because then the performance in transfer and non-transfer conditions would be equally accurate. If not abstract rules, what do people learn? And to what extent is such knowledge relevant to understanding linguistic knowledge? 
Despite the initial assumptions that people could indeed learn the abstract rules generating the stimuli they are exposed to (such as the underlying artificial grammar systems), gradually the prevailing views shifted to accounts stressing the role of the actually experienced exemplars. It turns out that the learning effects could be explained by matching similarity between test and training items stored in memory (Brooks, 1978; Vokey \& Brooks, 1992). Moreover, people do not necessarily need to remember whole training items to discriminate the grammatical and ungrammatical test ones above chance. It is enough that they remember fragments. For example in AGL people may notice certain bigram and trigram "chunks", such as PV, PX, VPS, TSS (as in Figure 1.1) and possibly also track which occur at the beginnings and ends of the strings. It has been shown that the prevalence of salient bigrams and trigrams in a given item predicts how likely it is to be classified as grammatical, regardless of its actual grammaticality (Servan-Schreiber \& Anderson, 1990; Johnstone \& Shanks, 1999). Furthermore, training participants on just bigrams alone has been shown to lead to the same pattern of responses as training on longer strings, indicating that chunk frequencies are a strong predictor of participants' performance (Perruchet \& Pacteau, 1990). These findings suggest therefore a close relationship between the experienced stimuli and the resulting knowledge.

In numerous currently popular statistical accounts people's performance on implicit learning tasks is successfully simulated in connectionist models operating on chunks (Perruchet \& Vinter, 1998). One prediction could be therefore that humans are endowed with powerful statistical learning mechanisms (e.g. Saffran, 2003; Saffran \& Wilson, 2003) which track when features co-occur and how frequently. On the other hand, instead of being inferred from statistical computation, chunk formation may in fact be the primary mechanism resulting from the laws of memory. For example, because frequently repeated information is less prone to being forgotten than less frequent information, natural forgetting results in sensitivity to event frequency, without any statistical 'computation' (Perruchet, 2008 and other). On such a view, sensitivity to statistical regularities becomes a by-product of the selection of chunks due to the memory mechanism. Both interpretations explain learning as a result of reinforcement of information through accumulative experience with invariants and covariants in the input.

Can the learning revealed by experimental paradigms other than AGL also be explained as essentially statistical in nature and possibly operating on stimulus fragments such as chunks? Lewicki's et al. (1988) study has sparked a discussion in the literature 
(Perruchet, Gallego, \& Savy, 1990; Ferrer-Gil, 1994; Cleeremans \& Jimenéz, 1998), with ample evidence being presented in support of statistical over rule-based accounts. For example, Perruchet et al. (1990) demonstrated that participants become sensitive to the relative frequency of small sequences of two or three successive locations. Close examination of the rules revealed that back-and-forth movements were overall less frequent than other types. They also never occurred in the last three trials within each block of the main training trials, but did occur in the random trials and therefore could have been responsible for the longer response times on those. However, these findings are at odds with Ferrer-Gil (1994) who discussed the disappearance of the effect under transfer to different sequences based on the same rules. The distribution of target movement types during the transfer was almost identical with the training phase, including the occurrence rates of back-and-forth movements. This indicates that target movement frequency cannot account for all learning effects emerging in the SRT procedure. Cleeremans and Jimenez (1998) attempted to reconcile these different findings in their connectionist Simple Recurrent Network (SRN) model, but conceded that if the simulation and human participants' data were to match more closely one would need to posit some explicit strategies that people could develop but that the model could not. Overall, regardless of whether the knowledge gained in SRT tasks consists of sequence fragments or unconscious sensitivity to transition probabilities between single events, the crucial factor affecting the performance on each trial seems to be the degree of overlap with the prior material.

Invariant learning has also been re-analysed in a similar vein. In McGeorge and Burton (1990) the stimuli which contained the invariant digit ' 3 ' also had proportionally fewer cases of repeated digits than those without it. Consequently, instead of only accepting the items with ' 3 ' participants could also be rejecting ${ }^{2}$ those containing repetitions. Wright \& Burton (1995) showed that this indeed explained at least part of the original results, which provides further evidence that in implicit learning tasks participants' knowledge is chiefly driven by similarity to at least fragments of the experienced stimuli, rather than by abstract rules.

Sensitivity to repetitions in fact has been demonstrated to be another key factor, next to sequence fragments or chunks, underlying implicit learning and transfer. Knowlton and

\footnotetext{
${ }^{2}$ Rejections may rely on explicit knowledge (Ellis, R, 2005); if performance was chiefly driven by rejections it would predominantly be explicit, which might be the case here because subjective awareness judgements revealed a high proportion of explicit responses.
} 
Squire (1996) suggest that this is what permitted amnesics to perform similarly to a healthy control group in their artificial grammar learning tasks, including transfer to a different letter set. Because amnesics could not rely on declarative knowledge of permissible chunks, as evidenced by their poor chunk recognition memory, the authors concluded that apart from chunk knowledge, people must also pick up "some rules about permissible locations of letter repetitions, alternations, or dependencies between different parts of the letter strings" (Knowlton \& Squire, 1996, p.179). Sensitivity to repetition or alternation could be just a case of statistical learning leading to formation of highly salient chunks, or, as some behavioural and neuroimaging research may suggest, an altogether separate mechanism (Tunney \& Altmann, 2001; Lotz \& Kinder, 2006; Dominey \& Ramus, 2000), perhaps less tied to the particular surface form features.

Because amnesic patients cannot rely on their declarative memory for chunks, repetitions are for them a more salient aspect of the stimuli. Healthy participants, on the other hand, can rely on both chunks and repetitions, whenever possible. In general, in different experimental conditions one aspect can be more salient than another and therefore exert a stronger influence on participants' behaviour. For example, in non-transfer conditions, both chunks and repetitions are relatively easily recognisable. In transfer, however, chunk knowledge requires mapping between elements based on their frequency of occurrence in different locations. Repetition patterns, on the other hand, are easier to identify regardless of the stimulus type and therefore a more likely basis of participants' performance under transfer conditions than chunks. Indeed, studies where the chunk-related and repetitionrelated factors are controlled, confirm that it is the repetition structure which is the primary basis for transfer (Scott \& Dienes, 2010 ).

The overall account of the transfer mechanism has implications for the research discussed in this dissertation as it directly addresses the question of the extent to which implicit knowledge is determined by the experienced material. Repetition patterns are clearly less dependent on particular surface features than chunks, but are they modality-independent in the same way as linguistic knowledge is modality-independent? After all, the hallmark of linguistic knowledge is its abstractness, understood as the property which allows speakers to both effortlessly comprehend and produce word combinations, in spoken or written form, bearing no similarity to previously encountered utterances. People can use language creatively. Therefore implicit learning is only an appropriate account of language acquisition inasmuch as it explains knowledge transferability to new vocabularies. Could transferability 
be an indication that implicit learning develops some knowledge which is not dependant on surface features of the stimuli?

Conway \& Christiansen (2006) tested this question directly. They employed a dualgrammar crossover design in which participants were simultaneously trained on two different artificial grammars in different modalities, one using colour sequences (visual) and the other using tones (auditory). Later they were informed that the items had been generated by a system and given a test with either a set of colour sequences or tones, half of which were grammatical with respect to one grammar and half with respect to the other. It was hypothesised that if implicit learning led to modality-independent knowledge then the test sequences generated by the two grammars would appear equally grammatical to the participants. On the other hand, if the statistical regularities were tied to the sense modality, people would only accept as grammatical those structures which had been presented in the same modality in training. One group of participants was only exposed to one grammar to provide a unimodal baseline measure. The results showed participants accepting fewer items from the grammar of the other modality than from the grammar of the same modality, which led the authors to conclude that the learning was modality-specific, and therefore the knowledge underlying implicit statistical learning must be closely tied to the sensory and perceptual features of the material. They suggest that the learning effects reported may rely on perceptual fluency, which means the items are processed more easily because similar ones had been processed before. Finally, the authors indicate that: "the ability to learn abstract principles and transfer them to new domains certainly appears to be a hallmark of explicit cognition; it is much less clear, especially in light of the current data, whether it is also a hallmark of implicit learning” (Conway and Christiansen, 2006, p. 911).

A strikingly different proposition was put forward by Scott \& Dienes (2010), namely that it is mostly implicit transfer that leads to accurate performance, and conscious, explicit judgements are often influenced by factors which may decrease transfer accuracy. Their study involved a careful analysis of the role of various structural features of the stimuli as well as subjective awareness measures on participants' decisions in an AGL task. The test phase was also combined with a clarification task to assess perceptual fluency. The authors found that although perceptual fluency influenced participants' decisions, it could not have been the key factor responsible for transfer. Perceptual fluency was related to the conscious feeling of familiarity, defined as "the subjective feeling of oldness elicited by a stimulus", which in turn correlated with the likelihood of the item being endorsed as grammatical. It did 
not, however, predict accuracy. Accuracy only reached significance $(60 \%)$ in the answers indicated as pure guesses, where familiarity contributed less than in other responses. The factor identified as being associated with these accurate guesses was 'novel chunk proportion', namely the proportion of bigrams and trigrams within a test string which did not appear in training.

These findings suggest therefore, contrary to Conway \& Christiansen (2006), that not only unconscious transfer is possible, but also that the unconscious might actually "outperform" the conscious. A natural question now is what could account for the disparity between these findings and those of Conway \& Christiansen (2006)?

A question which may arise with regards to the findings in Conway \& Christiansen (2006) pertains to the account of the transfer decrement effect. Cross-modal transfer is naturally expected to lead to lesser accuracy than a non-transfer condition. Therefore, if the stimuli in test were presented in one of the two modalities from training, it is expected that participants would accept more items from the tested modality than the other, which is precisely the reported pattern. However, perhaps the key issue pertains to the fact that there was no difference in performance between the dual modal and the unimodal baseline group. Participants who were exposed to both the visual and auditory grammars combined scored at the same level as those who were exposed to the visual grammar alone. For the uni-modal group, test items generated by the second grammar were ungrammatical in the conventional sense, whereas for the dual modal group all items were grammatical according to one or the other grammar. The fact that the two groups performed comparably is surprising, because if there had been at least a small amount of transfer then the dual modal group should have shown lower accuracy than the single modal group (because they would have tended to accept things from the 'other' grammar). This absolute lack of transfer in the dual modal group is surprising, and suggests involvement of a conscious strategy.

Comparing this study with other artificial grammar experiments demonstrating transfer, there seems to be a crucial procedural difference. In the typical AGL task people are trained in one modality and then told that there was a regularity, and that they would be presented with stimuli in another modality which might or might not follow the same regularity. They are, in other words, told to transfer their knowledge. It is not clear whether participants here were instructed in this way. If not, it was natural for them to judge visual stimuli according to whether they conformed to the pattern underlying the visual stimuli in training. The lack of transfer may indicate this was the case. Unfortunately, participants' 
strategies are not known because the procedure did not include any measures of awareness (despite which the authors concluded the learning was implicit).

What the study shows, therefore, is that when making grammaticality judgements people can zoom in on the training experiences which they think are relevant -i.e. strings are judged grammatical with respect to some knowledge base. This does not rule out effects of implicit knowledge of the structure of that knowledge base, but does indicate that there is no interference between different grammars in different modalities. Transfer across modalities may require an analogical process when making judgements, unhindered by intentional adherence to an explicit strategy (here participants may have assumed they were to judge stimuli with respect to the grammar in the same modality). Could it therefore mean that participants' potential explicit strategies hindered the transfer of implicit knowledge?

This would be in accord with the conclusions put forward by Scott and Dienes (2010). However, in their study 'chunk novelty' was identified as the key factor of unconscious transfer. It is not stated, however, whether new chunks in test were used mainly in ungrammatical items. If that were the case, participants might consciously have recognised them as new fragments and discriminated the strings as ungrammatical, and have done so while being less confident of such decisions than when consciously recognising something they had seen. This would augment the accuracy of the 'guess' judgements. It has been proposed in the literature that rejecting ungrammatical items primarily relies on explicit knowledge (Ellis, R. 2005).

The debate about the conscious/unconscious nature of transfer remains unresolved. But what becomes immediately apparent is the fact that the implicit learning mechanism is highly sensitive to many factors. Particular task demands or even instructions may focus participants' attention on those features which later determine what they learn and which aspects of the structure of the stimuli they will be sensitive to in a subsequent test (Wright \& Whittlesea, 1998). Simply the manner of stimulus presentation may turn out to be critical in making the relevant features salient. For example, groups of geometric shapes determined by an artificial grammar have been shown to induce implicit learning when presented together but not when presented as sequences of individual shapes (Pothos \& Bailey, 2000). Prior knowledge may also exert a top-down influence on what people learn: e.g. in Brockmole, Hambrick, Windisch, \& Henderson (2008) only advanced chess players showed contextual cueing when the context for learning was provided by the repeated chessboard arrangements, the meaningfulness of which depends on the observer's knowledge of the game. Even 
temporary explicit expectations may interfere with learning. Pothos (2005) performed a study in which the stimuli were sequences of cities on the route of a travelling salesman. When an explicit expectation that the salesman would choose shorter rather than longer journeys was violated, learning was inhibited.

To summarise, the field of implicit learning research has been rapidly growing since its beginning in the late 1960s, with numerous methods developed to measure whether and how implicit learning may occur. So far it has become apparent that what people learn is determined by the information they process or are able to process (such as in the case of healthy vs. amnesic participants), which in turn is determined by what is salient enough in the input to be taken in. Also, any learning situation is unlikely to be "process pure", but will actually involve a mixture of both explicit and implicit learning, each of which may rely on different aspects of the stimulus material, to a different extent. Chunks, for example, may be primarily explicit, while the analogical mapping or sensitivity to repetition patterns may, at least in part, be implicit.

To what extent could implicit learning of this kind, based on surface form regularities, be involved in language acquisition? Could it provide an accurate explanation of the emergence of linguistic knowledge?

\subsection{Form-level implicit learning in language acquisition}

Research on implicit learning in first and second language acquisition to date has revealed similar phenomena to the ones described above. For example, both adults and children (8 months old) have been shown to use chunking in segmentation of continuous, unfamiliar speech into words (Saffran, Newport, \& Aslin, 1996a; Saffran, Newport, \& Aslin, 1996b).

While listening to a stream of seemingly random syllables (e.g. $b a-b u-p u-b u-p a-d a-d u-t a-b a-$ $p a-t u$ - $b i-p i-d a-b u-t u-t i-b u \ldots)$ they rapidly acquired a sense of recurring fragments which could be regarded as words (e.g. babupu, bupada, dutaba, patubi, pidabu, and tutibu). Because in these experiments the only cue to word boundaries was a relatively low transition probability between syllables (there were no prosodic cues), the implication is that people unconsciously tally transition probabilities between elements in the input. In a similar vein, 
the acquisition of phrase structure could be based on the fact that transitional probabilities between words within a phrase are higher than between phrases (Morgan, Meier, \& Newport, 1989; Thompson \& Newport, 2007). Although neither participants' strategies nor awareness were measured in the above tasks, it may be assumed that at least in such young children the learning was implicit.

Statistical learning effects based on the distribution of sounds have also been demonstrated in discriminating phoneme categories in both children (Maye, Werker, \& Gerken, 2002) and adults (Maye \& Gerken, 2000). In these tasks participants were required to indicate whether two words were the same one repeated or two different words in the artificial language they had been exposed to. Although participants' strategies were not monitored, it is not unlikely that in a task like this - which addresses very fine perceptual contrasts - many of the participants' decisions were actually guesses (in fact, they were encouraged to guess in cases of uncertainty), which means that to some extent the learning effects shown could have been implicit. There is less such uncertainty in the studies of Dell, Reed, Adams, \& Meyer (2000) and Warker \& Dell (2006) who demonstrated implicit learning effects in adults learning phonotactic constraints and orthography. Since the learning was assessed on speech errors, which are produced unintentionally and automatically, the authors concluded that it was implicit.

Determining the extent to which a given learning effect is implicit or explicit is not of negligible importance. In light of the discussion in section 1.3, explicit and implicit learning mechanisms may be sensitive to different aspects of the stimulus material, and importantly, as in Scott and Dienes (2010), explicit strategies may sometimes dominate and yield different performance than if implicit learning was proceeding in isolation. For example, it may be the case that the difference between the two learning modes could explain such discrepancies as reported in Baese-Berk (2010). Two groups were trained on a novel phonetic category contrast using the same task as Maye \& Gerken (2000). One group followed a perceptiononly procedure, and the other a perception-and-production procedure, where they were asked to explicitly repeat tokens. In the following phoneme discrimination task, the perception-only group outperformed the perception-and-production one, suggesting that production training can disrupt perceptual learning (although marked individual differences were observed as well). It is rather likely that participants in the perception-and-production group relied on explicit learning more than the perception-only group. A replication of these findings with additional measures of awareness could illustrate the relative contribution of explicit and 
implicit learning in production tasks. For the time being, it can only be concluded, as in section 1.3, that task demands highly influence the learning which may occur, be it implicit or explicit or, most likely, both.

Apart from chunking and tallying probabilities, sensitivity to repetitions has also been demonstrated to play a part in language acquisition, both first and second. For example, studies by Marcus, Vijayan, Bandi Rao, \& Vishton (1999) revealed sensitivity to repetitions among 7-month old infants. In the initial habituation phase streams of syllables were played, which followed either an ABA pattern (e.g. ga ti ga) or ABB (ga ti ti). Although the test items were composed of novel syllables (wo fe $f e$ ), infants attended longer to those which followed the same pattern they had heard. In a statistical learning paradigm (with no measures of awareness), Onnis et al. (2008) showed that relatively close repetitions of artificial 'words' in a speech stream also help adult learners to detect them. Furthermore, even form-meaning mappings are easier to establish when input is structured as partial selfrepetitions, as reported in a cross-situational learning study by Onnis, Edelman, \& Waterfall (2011).

These experiments suggest that form-level implicit learning may play a prominent role in the initial stages of language acquisition and that it may underlie such processes as early speech segmentation and phoneme extraction, which subsequently link to formmeaning mappings (Hay \& Saffran, 2011).

The above presented studies relied on artificial language streams. However, the picture is not complete until these findings are compared with similar investigations using natural language. These reveal that the language learning mechanism is more complex, with numerous top-down, mostly unconscious, influences from lexical information, the stage of language development or proficiency, and, at least in the case of L2 learning, from the preexisting grammar.

One example of such top-down effects is the influence of lexical information on phoneme perception in adult second language learners (Feldman, Myers, White, Griffiths, \& Morgan, 2011). Participants who heard the tah and taw syllables in distinct lexical contexts were more likely to treat the vowel sounds as belonging to different categories as compared to participants who heard tah and taw interchangeably in the same set of lexical contexts. These results show that adults alter their interpretation of acoustic variability on the basis of word-level information (unfortunately there were no measures of how conscious the performance was). 
Not only the mere linguistic context may have top-down effects on learning, but also specific languages may exert different top-down effects. For instance, Eckman \& Iverson, (in press) presented data showing that the differences in sound classification in the learner's native language (Korean versus Japanese classification of [s] and [J]) have different consequences for both the acquisition patterns and the error types produced in the course of learning the given contrast in L2 (English).

Also, stages of first language development in infants correlate with their predominant reliance on different statistical cues for learning semantic information. A study by Lany \& Saffran (2011) revealed that 22-month-old infants with smaller vocabularies generalised using phonological cues, whereas infants with larger vocabularies relied more on distributional cues, possibly due to having had more experience of weighing the importance of various types of statistical regularities in language. This suggests that language acquisition cannot be modelled purely in terms of statistics without considering prior knowledge or the amount of prior linguistic experience. Furthermore, Peperkamp, Le Calvez, Nadal, \& Dupoux (2006) illustrate that distribution of allophones of a given phoneme is hardly ever as clear-cut in natural language as it is made out to be in artificial language experiments. An analysis of a natural corpus revealed a very large number of near-complementary distributions not corresponding to existing allophonic rules. The authors propose an algorithm to account for this situation, which involves a linguistically motivated filtering mechanism, suggesting therefore an important role of prior linguistic knowledge in the statistical learning of phonology.

Together these findings suggest that purely statistical modelling does not always offer the most accurate account of language acquisition. Statistical modelling enriched with linguistically motivated factors (such as stages of development and prior knowledge) offers a more tenable approach. However, the extent of the influence of linguistic (and other) factors is not easy to detect and analyse. Implicit learning methodology provides one solution to this problem, by measuring knowledge unaffected by conscious, explicit strategies, working memory or general intelligence, which may potentially skew other experimental investigations addressing deeply automatic, unconscious knowledge - such as the knowledge of language.

Recent research has provided evidence that implicit learning is indeed highly related to language abilities. For example, Kaufman et al. (2010) reported a study investigating the association of individual differences in implicit learning with numerous cognitive variables, 
such as psychometric intelligence, working memory or academic performance. They found that implicit learning was not related to overall intelligence or working memory. It turned out to be related only to academic performance on foreign language exams, and two aspects of psychometric intelligence: verbal analogical reasoning and processing speed - arguably, crucially linguistic traits. ${ }^{3}$

Also, language disabilities have been linked to impaired implicit learning. For example, there is a longstanding debate in the literature over whether dyslexia is linked to a breakdown of implicit sequence learning, with conflicting evidence being reported. Dyslexia is a cognitive disorder affecting reading and writing skills, with the absence of an intelligence deficit or other cognitive disabilities. For this reason it was hypothesised that its underlying cause does not relate to conscious learning processes, but rather the implicit learning mechanism underlying abilities relying on sequence processing, such as the ability to read or write. In a convincing recent study and analysis by Jimenez-Fernandez, Gracia Vaquero et al. (2011) the authors argue that those studies which claim to demonstrate successful implicit sequence learning among dyslexics are based on materials which allow participants to use complementary strategies. For instance, in a number of studies the events in sequences were not balanced for frequency, allowing participants to report based on event familiarity. Certain sequences also involved regularities which were simple enough to decipher consciously. When event frequency and cue saliency are controlled, only healthy control participants and not dyslexics show learning. These findings support the general conclusion that implicit learning is related to language and it is driven by those aspects in the stimuli which are salient enough to be processed. It is therefore related to people's processing abilities.

All in all, studies of language acquisition provide a natural reflection of what has been discovered in the field of psychology. Language acquisition, just like the domain general mechanism of implicit learning, is determined by such factors as form frequency and saliency, lending itself to statistical modelling. In this sense language can be considered "a mirror of the mind"4. It is not, however, a model of it ${ }^{5}$. Language exerts top-down effects and has discrete stages of development of which learners are not conscious. Implicit learning research clearly offers a promising, empirical approach to better understanding the underlying mechanism of language acquisition. With the variety of methods developed to investigate

\footnotetext{
${ }^{3}$ Interestingly, the authors also report a relation between implicit learning and aspects of personality including intuition, openness to experience and impulsivity.

${ }^{4}$ Chomsky (1993), p. 34.

${ }^{5}$ Ibidem.
} 
implicit learning - AGL, SRT, invariant learning, contextual cueing - it has already deepened our understanding of the processes involved in the acquisition of knowledge in general. However, most of the current research has focused on regularities in perceptual, mostly visual forms. Language, on the other hand, operates on intrinsically meaningful units. Can the mechanism of implicit learning be better understood when applied to meaningful material?

\subsection{Higher-level (semantic) implicit learning}

So far, considerably less attention has been devoted to implicit learning of regularities at an abstract level of representation, not pertaining to surface forms of stimuli. Initial attempts to demonstrate it date back to the times when many researchers believed implicit learning could lead to knowledge of abstract rules. Showing the learning of abstract, meaningful generalisations was seen as a way of confirming that intuition.

Bright \& Burton (1994) documented a study in which they showed learning of a nonsalient invariant feature. Participants saw a series of 30 clock faces and simply wrote down the times. They were not informed that all of the times fell between 6 and 12 o'clock. In an unexpected test they were presented with pairs of clock faces and asked to choose the one which appeared in the previous phase. Even though in fact all of the clock faces were new, participants showed a preference for the ones displaying the times between 6 and 12, even when no awareness of the regularity was reported in the final debriefing. Crucially, the authors showed that the effect persisted under a transfer condition when analogue clock faces in training were changed to digital ones in test. Initially this was taken as an abstraction of the rule and demonstration of knowledge that was independent from the visual similarity to the experienced exemplars. Subsequently, however, this conclusion has been revised.

Interestingly, in Bright and Burton (1994) participants showed no preference for the actual seen clock faces when they were included in the test (Experiment 4). Initially, this was taken as strong evidence for abstraction and form-independence of the resulting knowledge. However, Newell \& Bright (2002) provided a revised explanation, namely that participants were more likely to encode the times in a rough manner, rather than extract the "6 to 12 " rule, and judged test items with regards to how similar they were to the training ones stored in memory. In their replication study, they constructed test pairs with varying degrees of intra- 
pair similarity. Test pairs with a low similarity between times were separated by 6 hours, e.g., 8:30 vs 2:30, those with a medium similarity were separated by 3 hours, e.g., 4:30 vs 7:30, and those with a high similarity were separated by 1 hour, e.g., 5:10 vs 6:10. If the originally proposed rule abstraction was the case, the degree of intra-pair similarity should not be of importance, and provided that one time in a pair fell between 6 and 12 it should naturally be preferred. Alternatively, if the learning effect was driven by similarity matching, then selection of the invariant in the 1-hour pairs would be lower (the times would seem equally close to the original item) than in the 6-hour pairs. The latter turned out to be the case, suggesting that the performance on such non-salient invariant learning tasks, just like in other implicit learning tasks - including the form-based ones - was driven to a greater extent by overall similarity than by an abstract rule. For further examples and discussion of invariant learning see Berry \& Cock (1998).

Such findings combined with the mainstream results about the implicit learning mechanism - its reliance on chunking, repetition structure and statistical regularities in the input - led to a decline in the popularity of research addressing abstraction/generalisation. Recent years, however, have seen a gradual revival of interest in these issues, focused not necessarily on rule abstraction but rather on the formation of conceptual knowledge. It is known that existing conceptual representations can be activated without conscious involvement, as in, for example, semantic priming experiments (Neely, 1977), arithmetical processing (Van Opstal, de Lange, \& Dehaene, 2011), or intuitive gestalt judgements (Bolte $\&$ Goschke, 2008). The controversial question is whether unconscious conceptual knowledge can not only be accessed without awareness, but whether it can also participate in novel associations.

The current demonstrations of implicit learning involving abstract representations, although scarce, may be divided into two types. The first addresses the question of whether new associations between existing concepts can be formed implicitly, and the second whether connections between novel forms and (pre-existing) concepts can be established without awareness. Both of these types of learning are relevant for language acquisition. 


\section{Abstract sequences}

An example study of the first kind, involving learning a thoroughly conceptual sequence is Goschke \& Bolte (2007). Participants named pictures of objects, e.g. table, shirt, nose, horse, trousers, ear. What they did not know is that the pictures followed a repeating sequence based on their underlying category (furniture-clothing-body part-animal-clothingbody part). After some exposure people were faster to name new objects when the object sequence followed the category sequence than when it did not, thereby showing generalisation. The pattern remained significant when taking into account only those participants who had no awareness of the underlying regularity based on the follow up sequence reproduction and recognition tasks and in comparison with a control group. Learning of category sequences is most likely based on forming gradual associations between category representations which are repeatedly activated in succession. As a result, processing an exemplar from one category leads to preactivation of the next category, which speeds up naming of objects coherent with the preactivated category, and delays naming of those incoherent with it (see Figure 1.3).

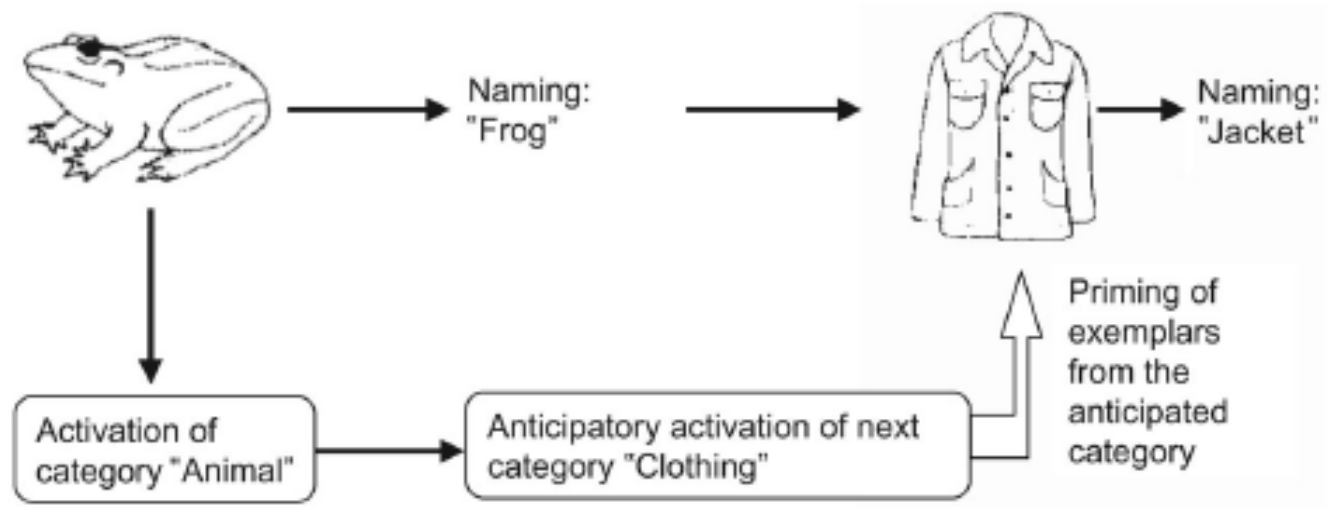

Figure 1.3. Schematic model of the cognitive processes underlying performance in the serial naming task (from Goschke and Bolte, 2007).

Brady \& Oliva, (2008) show a similar effect for pictures of scenes which followed a predictable sequence of categories (e.g. mountain-building-kitchen-forest), including transfer to word equivalents of the category sequences. Participants initially performed a simple task 
(repetition detection) on a stream of pictures. They were then tested on pairs of triplets of pictures, one of which followed the category sequence from the training, while the other did not. The participants' task was to choose which triplet they felt was more familiar. They were able to discriminate the two types above chance, even when the exact pictures had not appeared in the combinations presented but the categories to which they belonged had, and while reporting no awareness of the category sequences. These studies show that people can track sequential regularities at conceptual levels, rather than just the superficial level of letter identity (in artificial grammars) or spatial location (Lewicki et al, 1987; 1988). This may be quite beneficial in the daily interactions with the world since, as Brady and Oliva, suggest, it reduces information redundancy, which is ubiquitous in the daily organisation of objects and their locations.

\section{Form-meaning connections}

One of the early studies showing that people are sensitive to covariation at different levels of representation (form-level and a higher conceptual level) is Lewicki (1986). He presented participants with a series of photographs of short and long-haired people (visual forms) together with short auditory descriptions describing the people's characters (concepts). Unbeknown to the participants, the long-haired people were all 'kind', while short-haired people could be more accurately described as 'capable' although neither of these precise words appeared in the descriptions. The participants' subsequent forced-choice judgements (e.g., 'capable' - yes/no) of new pictured people, as well as their response latencies, indicated that they indeed learned this covariation. They did so without being able to report any dependencies in the stimuli. Because neither of the words 'kind' nor 'capable' appeared in the descriptions, participants must have extracted the relevant general notions about characters of the presented people and associated them with hair-length. This reinforces the notion that certain regularities based on semantic, conceptual generalisations are learnable without awareness.

Further interesting demonstrations of this phenomenon come from the area of visual perception and the derived attention paradigm. Lambert \& Sumich (1996) showed that participants can become sensitive to the predictive relationship between the semantic category of words appearing on screen (living or non-living) and the position of a target dot 
located in a subsequent display, without awareness of the regularity throughout the experiment. More recently, similar findings were obtained using the contextual cuing paradigm (Goujon, Didierjean, \& Marmèche, 2007; Goujon, 2011). In Goujon et al. (2007) participants had to search for a target (a number 13 or 28) in a display containing other numbers as distractors. The time the participants took to indicate whether the target was on the left or right of the screen was recorded. What they didn't know was that whether the target was on the left or right of the screen was predicted by whether the distractors were odd or even numbers. After some time performing the task, response times on such predictive trials were faster than on trials where the distracters were a mixture of odd and even numbers. This was the case even when the distractors were drawn from a different set of odd and even numbers from those encountered in training. Participants apparently had no awareness of the regularity, so they appeared to have unconsciously abstracted the relationship between odd/even numbers and the target position. This, again, appears to be a case of learning an abstract regularity based on a semantic generalisation.

Goujon et al (2011) report a similar effect using real-world scenes, in which the category of the scene (e.g., a bedroom or a living room) predicted the location of a target. Interestingly, though, the effect only arose when participants were asked to categorise a preview scene (even when it was different from the subsequent search scene) or could at least preview the search scene before the visual search trial, indicating that some processing enhancement may be necessary for such semantic implicit learning effects to occur.

What is immediately striking in the presented studies is how readily people generalise when the experimental stimuli are intrinsically meaningful. Since the learning material pertains to abstract, conceptual categories, they naturally (possibly automatically) generalise. An obvious question at this stage is - do similar phenomena take place in language? Language, after all, does operate on intrinsically meaningful elements - words ${ }^{6}$.

\subsection{Higher-level (semantic) implicit learning in language acquisition}

To a large extent language research has mirrored the above findings. Here too there are demonstrations of implicit learning of abstract regularities, such as those governing word

\footnotetext{
${ }^{6}$ The smallest meaningful units in language are morphemes, but this dissertation mainly concerns regularities based on content words.
} 
order in a sentence, as well as form-meaning connections, the latter being most relevant to the research reported in this dissertation.

\section{Abstract sequences}

Rebuschat \& Williams (2011) report a study showing implicit learning of abstract sequences in language. Participants were exposed to sentences using English words and, unbeknown to them, German word order, e.g. George repeated today that the movers his furniture scratched. In a surprise grammatical judgement task on new sentences containing different words, the sentences which followed the grammatical patterns from the training were accepted at higher levels (above chance and above a control group with no training) than the ungrammatical sentences. Therefore, clearly there was rapid incidental learning of abstract word order patterns. Participants most likely incidentally extracted template representations of sequences based on word categories, which were abstract enough to allow transfer to new lexis. However, analysis of the ungrammatical test items suggested that there was no learning of abstract word order rules as such (e.g. verb final in subordinate clauses). Williams \& Kuribara (2008), and Williams (2010) report similar findings, again showing incidental learning of category sequences, rather than generalised word order rules. These results are consistent with evidence for learning chunks rather than abstract rules in artificial grammar learning (see section 1.4, above), but here learning is at the level of abstract grammatical categories, and hence is reminiscent of learning sequences of semantic categories (Section $1.5)$.

\section{Form-meaning connections}

Initial reports cast doubt on the possibility of learning form-meaning connections implicitly. DeKeyser (1995) utilised an artificial language with rich inflectional morphology for marking gender, number and object role. In an extensive study involving 20 learning sessions, participants were initially asked to indicate whether a given sentence correctly described a picture or not, e.g., Bep-on warufk-at rip-us (Worker-PL build-PL house-OBJ). The subsequent test involved a production task, where they had to describe pictures using the novel language. It turned out that they were only able to do so when it was possible to use 
stem-inflection combinations which had occurred in training. When items required novel stem-inflection combinations they performed at chance, indicating they did not learn the general, semantic properties of the inflectional morphemes. However, the sentence-picture training task that was used in this study did not appear to require participants to pay attention to the inflectional morphemes. Given the importance of noticing form for learning (see Section 1.2) it is therefore not surprising that no learning of form-meaning connections was obtained.

Additionally, the findings by Leow $(1997 ; 2000)$ challenge the idea that formmeaning connections can be established without awareness. His studies involved the learning of irregular (stem-changing) verbs in Spanish. Participants were solving a crossword puzzle during which they were required to report any thoughts coming to their mind. Interestingly, only those who commented on the changes of stem in the critical items later showed significant gains between pre-test and post-test. The study therefore suggests that there is no learning without 'noticing' (see next section for discussion). Before Leow's experiments, Schmidt had also argued for the same conclusion on the basis of Schmidt \& Frota (1986) where he performed a case study of his own learning of Portuguese over a 6-month period in Brazil. He documented all aspects of the language he became aware of in a detailed diary, which also included recordings and transcriptions of his conversations with the natives. Schmidt noted a strong tendency for the things he noticed in the input to later appear in his productions, which led him to advocate that conscious 'noticing' should be crucial for learning (as mentioned in Section 1.2).

More promising results come from the research by Williams (2005) and Leung \& Williams (2011, 2012). In Williams (2005) participants were first taught a system of four determiners: gi, ro, $u l$ and ne. They were told that they had a similar role to the English definite article but they were also sensitive to the distance between the speaker and the object. In the system, $u l$ and ne were used for distant objects, whereas gi and ro were used for the near ones. They were not told, however, that the choice of the determiner also depended on animacy of the following noun. Animate objects required either gi (near-animate) or $u l$ (faranimate), while inanimates requried ro (near-inanimate) or ne (far-inanimate). The participants were shown correct sentences (e.g. I heard the sound of ul monkey in the tree; I knocked over ro cup and coffee spilled on my book) and were trained on the distinction between far and near objects. While reading the sentences they were also asked to form mental images of the described situations. After training, they were given a test, where they 
had to choose between two possible determiners. In the test, familiar words appeared in new contexts, e.g. The art collector went to Greece to collect ul / ne vases (correct answer "ne vases"). Neither $u l$ or $n e$ had occurred with the noun vase during training. Those who claimed not being aware of the relevance of animacy to article usage during training or testing phases answered accurately $61 \%$ of the time, which is significantly above chance, showing compelling evidence for implicit learning of form-meaning connections.

Leung and Williams $(2011,2012)$ corroborated these findings and showed evidence for the learning of other meaningful distinctions between determiners, such as whether the noun describes an agent or a receiver of an action (thematic roles: agent versus patient). At the same time, they failed to show learning of a non-linguistically motivated distinction concerning the relative size of objects. They hypothesised that implicit learning of formmeaning connections may be constrained by prior assumptions about potentially encodable distinctions. Bickerton, (1999) proposed a list of semantic features which could potentially be grammaticised in language, including "number, gender, person, sense, modality, aspect, specificity, possession, object-type (e.g., long/thin versus spherical), transitivity, agency, grammatical function, and so forth" (p.204). Bickerton suggests that without such a list language would be unlearnable due to the infinite number of potential semantic distinctions.

The material in this dissertation continues the research from this point. The potentially grammaticisable semantic distinctions could facilitate learning of functional morphemes, such as determiners. But what about non-grammaticised semantic relations, such as those between content words, e.g., verbs and their following nouns? Semantic distinctions relevant to a set of nouns which may appropriately follow a given verb are also potentially infinite. Would implicit learning occur in this context as well?

Initial support for the idea that people may indeed form semantic generalisations on content words comes from the study by Guo et al. (2011) examining implicit learning of semantic prosody. Semantic prosody is a pragmatic phenomenon wherein a word has a positive or negative connotation. A grammaticality judgement task combined with subjective measures of awareness revealed participant's unconscious knowledge of the semantic prosody of 6 novel words, which were based on existing English words. However, this result remains controversial as it is not clear whether participants identified the direct translation equivalents of the words and simply applied the prosody of those. This could have been facilitated by the fact that the target words belonged to different syntactic categories and were easily distinghishable, e.g. tinberly - totally, lampit - promote, pooth - career. Furthermore, 
semantic prosody may also be universal in the Bickertonian sense, whereas semantic preferences could potentially be infinite. Nonetheless, these encouraging results clearly demonstrate that more research is needed in the area of unconscious semantic generalisation.

\subsection{Types, tokens, syntagmatic and paradigmatic learning - the case of semantic preferences}

A semantic preference can be understood as a particular type of collocation, where collocation refers to higher than chance co-occurrence of two or more words. Collocates sound natural together and substituting one of them with a near-synonym results in a loss of naturalness for native speakers, for example in English it is better to say fast car and fast food, rather than * quick car or *quick food. Conversely, it is more natural to say quick glance and quick meal instead of *fast glance or *fast meal.

It has been traditionally proposed that collocations reflect syntagmatic relations between words, therefore related to their surface structure, rather than paradigmatic relations - regarding their meaning. "Meaning by collocation is an abstraction at the syntagmatic level and is not directly concerned with the conceptual or idea approach to the meaning of words. One of the meanings of 'night' is its collocability with 'dark'..." (Firth, 1957)

This claim has been reinforced within the popular framework of statistical modelling of language learning. Words co-occuring with each other more often than chance can be perceived as chunks, similar to chunks in AGL (Ellis, 2001, 2002, 2003, 2006; Hoey, 2005; Sinclair, 1991, 2004) Chunks are believed to be formed due to the principle of associative learning which Ellis (2001) calls the "Law of Contiguity" according to which "objects once experienced together tend to become associated in the imagination, so that when any one of them is thought of, the others are likely to be thought of also" (James, 1890, quoted in Ellis, 2001). In this way, long-term memory becomes 'tuned' to frequent collocations. When the same items are encountered again in the subsequent input they are recognised as a chunk, which further reinforces their long-term representations.

However, syntagmatic regularities may not be the optimal, or the sole way of accounting for the existence and acquisition of semantic preferences. After all, new collocates can be freely generated, as long as they follow implicit assumptions regarding applicable semantic sets. In the example from section 1.2 of this chapter, knowing that pack collocates 
with dog, hounds, wolves etc. while swarm with bees, mosquitoes, bats, naturally suggests other animals which would be appropriate in either set. It makes sense therefore, to predict that the existence of such semantically preferred sets of collocates involves abstraction at a level higher than form. Intuitions about collocability form an important aspect of attaining a native-like command of a language. In fact, it has been suggested that the same process that guides formation of collocations (as one example of formulaic language) may be central to language acquisition in general (Ellis, 2001). In spite of the significance of this area, so far little empirical research has been reported.

Two types of chunks may be distinguished: types and tokens. While tokens refer to particular words or phrases, types indicate the number of distinct lexical items which can be substituted in a given slot in a construction. Tokens would include, for example, the fixed, non-decomposable idiomatic expressions, such as kick the bucket, which cannot be broken into parts without a loss of meaning. *A bucket was kicked by the poor man loses idiomaticity. In such cases the meaning of the whole is not composed of the individual words' meanings and the sequential order must be kept intact. Types, on the other hand, offer a range of options within a construction, and can be illustrated by various sorts of collocations, from non-compositional but decomposable ones, such as sharp contrast, bitterly disappointing, heated argument, where collocates can be separated, and where also the meaning of a construction goes beyond the sum of its parts, to compositional and decomposable ones like: fast car, juvenile delinquent which can be separated and the meaning of a whole is indeed the sum of its components. Within the statistical language learning framework, however, decomposability is not an issue, since chunking is claimed to be a purely syntagmatic process. Crucially, chunking is believed to happen implicitly, without learners' intention or conscious monitoring. "To the extent that language processing is based on frequency and probabilistic knowledge, language learning is implicit learning" (Ellis, 2002).

So far, researchers have focused on token chunks. For example, the fact that fixed expressions, specifically binomials such as "fish and chips", are stored as a single unit has been convincingly demonstrated by Siyanova-Chanturia, Conklin, \& Schmitt (2011) However, as Ellis (2002) points out there is a growing number of studies according to which it is the type chunk frequency which drives human categorisation processes, and with them language acquisition. He quotes a study by Barsalou, Huttenlocher, \& Lamberts (1998) which reports a series of experiments on face recognition. It has been demonstrated that rather than 
track individuals across events (token frequency) people form representations of them, which they then apply in categorisation. In other words, they form abstract types. Human categorisation may be therefore more sensitive to type frequency rather than simple token frequency. Ellis put this forward as a key issue for language acquisition.

Idiomatic expressions may be numerous in language, yet even more numerous are arrays of less fixed constructions. The examples are plentiful and may involve various degrees of abstraction around the set of collocates. In phrases such as beyond belief/description/doubt/question/recognition the noun that follows the verb, although different in each case, always indicates some "verbal process", and the meaning of the whole may be roughly paraphrased as "too great to believe/describe/doubt...". Schmitt (2010) describes such phenomena as "formulae with open slots", illustrating them with a longer construction: thinks nothing of . The slots are not restricted to specific words, yet impose more generalised, paradigmatic restrictions, namely the first slot requires an animate subject, and the second one an activity which is unexpected or unusual. So, while (5) is well-formed, clearly (6) and (7) violate the semantic constraints:

(5) John thinks nothing of running a marathon each year.

(6) *The cup thinks nothing of being plastic.

(7) * She thinks nothing of tying her shoelaces.

The existence of these variable expressions, using Sinclair's (2004) terminology, poses a challenge for learners of both first and second language. Yet what the above examples show, is that having a generalised notion of what meaning is required in a given slot in a construction may potentially be very helpful in determining which words can and cannot be used. Native speakers develop such generalised notions incidentally, and possibly are not always aware of precisely what identifies legitimate collocates. Is it possible for second language learners to develop such notions implicitly as well? 


\subsection{Methodological issues}

The difficulty concerning accurate operationalisation of implicit learning is partially a result of the evolution taking place in the definition of the concept itself. Frensch (1998) groups the numerous definitions which had been proposed by that time according to whether a lack of conscious awareness should pertain to the learning process or to the learning and retrieval processes. Postulating that it should pertain to both learning and retrieval helps to clearly distinguish between incidental and implicit learning. Incidental learning takes place when people 'discover' something although they had no prior intention to do so. A natural example of incidental learning could be realising the meanings of unfamiliar words while reading. In experimental conditions incidental learning refers to situations where participants are not informed about the coming test. Implicit learning usually refers to those incidental learning situations where participants also remain unaware of what they have learned. In the early studies, this was ascertained when people were unable to, post-experimentally, verbalise their knowledge (unlike the vocabulary example), hence the unconsciousness of retrieval. In sum, lack of intention to learn a particular thing combined with inability to verbalise what one has learned at the end, has often been assumed to indicate that the process occurred unconsciously.

The conscious or unconscious availability of the end-state knowledge, however, may not always necessarily be an indicator of the process which led to it. For example, implicit knowledge may be developed in an explicit process which starts intentionally (e.g. learning to play an instrument), and through repetition and practice, gradually becomes more automatic or proceduralised. Competent composers of music, for example, are not always consciously aware of the details of musical structure while composing a piece. One could say that over time it becomes "second nature" to them. So, at least some aspects of initially explicit knowledge, through practice and proceduralisation, may lead to a different kind of knowledge, which is no longer available to conscious introspection. The reverse situation, where initially implicit knowledge spontaneously becomes explicit, is also possible, in what can be called "insight". These processes are summarised in Figure 1-4. 
IMPLICIT LEARNING (incidental inductive learning)

\section{EXPLICIT LEARNING} (instruction, intentional induction)

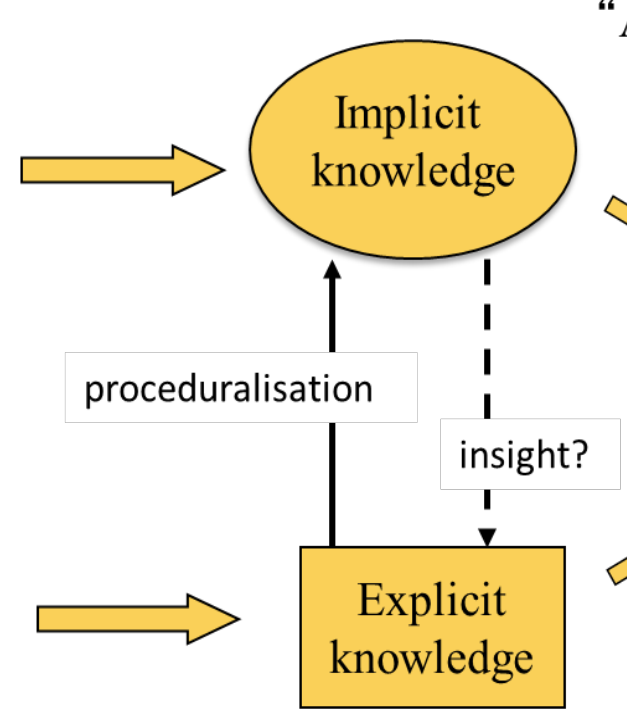

"ACQUISITION"

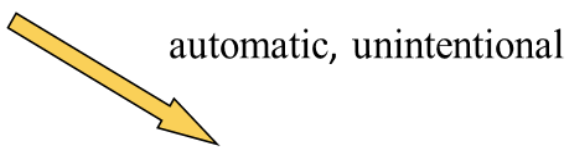

BEHAVIOUR

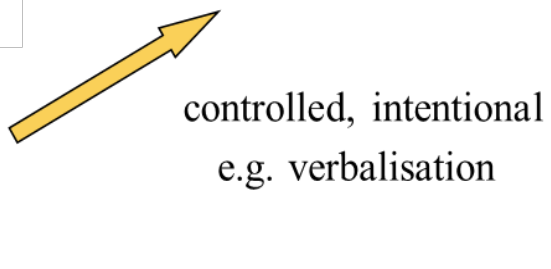

“LEARNING”

Figure 1.4. Relationships between implicit and explicit learning and knowledge (reproduced with permission from (Williams, 2012).

Since the process of implicit learning could lead to both implicit and explicit knowledge, the most promising approach to measuring it is to analyse people's awareness of a given regularity and analyse their behaviour during the learning process. This could be done either directly or indirectly. Directly, this can be performed by asking questions requiring introspection during the test and analysing only those responses which are not linked with explicit knowledge. Indirectly, implicitness can be assumed if people's behaviour displays established characteristics of an unintentional and uncontrolled process, such as automaticity, and can be further validated by a post-experiment verbal report. ${ }^{7}$

The endeavour of demonstrating learning without awareness becomes yet more challenging in light of the fact that the definition of awareness itself and its relation to attention as well as its role in learning in general remain contentious. While the debate remains unresolved, in implicit learning research it is best to establish functional implicitness, rather than absolute implicitness. Functional implicitness can be understood as occurring when we do not use whatever knowledge we have strategically. For example, in the case of social cognition, we may have a lot of explicit stereotypical knowledge about e.g. blond people or Polish (sic!) people, but not use it strategically. In the perception of art, experts

\footnotetext{
${ }^{7}$ Implicit behaviour will be automatic, but not all automatic behaviour is implicit.
} 
may possess much explicit knowledge about what makes a genuine kouros (ancient Greek sculpture) but still be influenced by intuitive, unverbalisable intuitions when making decisions $^{8}$. This further illustrates the dissociation between knowledge and performance. That is why it is important to establish what was driving participants' performance at the moment when learning effects were measured.

As has been signalled in part 1.3 and 1.4, determining whether the learning has been implicit or explicit in a given task is of considerable importance. Experiments may yield different interpretations when participants' awareness is taken into account compared to when it is not. Furthermore, establishing the level of awareness is also important in light of the debate concerning the very existence of the implicit learning phenomenon (section 1.7 of this chapter). Shanks \& St John (1994) proposed therefore two criteria which need to be satisfied for implicit learning to be legitimately demonstrated: the information criterion and the sensitivity criterion.

\section{Information criterion:}

The information participants are asked to provide on the awareness test must be the information responsible for the improved level of performance.

\section{Sensitivity criterion:}

The test of awareness must be sensitive to all of the relevant knowledge.

People may be consciously aware of more task-relevant aspects than an insensitive awareness test detects.

Below is a summary and brief evaluation of different methods which have been used to date to measure participants' awareness in implicit learning tasks.

\section{Verbal reports}

Method: In many classic experiments on implicit learning, the lack of awareness was established on the basis that participants were unable to report what they had learned immediately after the test (Berry \& Broadbent, 1984; Reber \& Allen, 1978).

\footnotetext{
${ }^{8}$ Gladwell, M. (2008).
} 
Pros: Even though the method has often been applied to experiments with complex systems underlying the stimuli, it is in fact more suitable when the learning target is simple enough that once acquired, learners would certainly be capable of reporting their knowledge of it. Post-experimental verbal reports also have the benefit that they do not introduce any extra awareness measures in the task, and thereby avoid prompting participants to intentionally guess the system or construct ad hoc hypotheses while performing the task. It is most appropriate as a method to double-check that the learning was implicit in procedures where the learning itself is measured by other means, e.g. reaction times, and only in such tasks where any conscious knowledge that might have arisen during the learning task would be unlikely to be forgotten by the time the test is applied.

Cons: The main problem with this method is that technically it can only reliably establish the state of resulting knowledge, rather than the level of awareness during the learning process. The overall crudeness of this method is what motivated Shanks \& St John (1994) to postulate their criteria. One potential issue here is the "retrospective problem". People indeed may have been aware of some relevant aspects of the task while performing it, yet forget them in the final debriefing (information criterion), or forget by the time the debriefing takes place. This could even be the case in amnesic patients - they may be rapidly forgetting what they had been fleetingly aware of during the task, leading researchers to mistakenly assume that they are capable of learning without awareness. Furthermore, the majority of experiments which relied on this method involved highly complex systems, for example artificial grammars, making any relevant knowledge difficult to verbalise and therefore easy to go undetected during the post-experiment checking of awareness (sensitivity criterion). This could be the reason why it is often assumed that implicit learning succeeds when the material is putatively too complex for explicit learning (Lewicki et al. 1987; Reber, 1989).

\section{Concurrent verbalisation}

Method: The method has been proposed by Leow (1997) and Rosa \& Leow (2004). It requires participants to report any thought that comes to their mind while performing a task.

Pros: If participants reported absolutely any thought that came to their mind during the task, the method would provide a straightforward answer to the question whether the given participant developed any task-relevant knowledge. It would also enable detecting the moment when the relevant knowledge emerged and tracking its development. 
Cons: Participants may have different reporting thresholds. Some of them might entertain ideas without mentioning them, or not describe low-confidence notions. Furthermore, this method has recently been shown to actually hinder learning, including explicit learning (Rebuschat, Hamrick, Sachs, Ziegler, \& Riestenberg, in preparation.), cf. verbal overshadowing.

\section{Subjective measures}

Method: This method was proposed by Dienes \& Scott, (2005) and Dienes (2008) and relies on the definition of implicit learning based on two criteria: the guessing criterion and the zero correlation criterion. According to the guessing criterion - implicit learning occurs when participants respond accurately when they believe they are literally guessing. The zero correlation criterion states that implicit learning occurs when participants' confidence in incorrect decisions is as high as confidence in correct decisions. The guessing and zero correlation criteria only reflect a person's subjectively experienced confidence in their judgement, as measured by ratings of confidence for each decision made. Dienes refers to this as judgement knowledge. This needs to be distinguished from the actual content of the knowledge that underlies a judgement - what Dienes has referred to as structural knowledge. For example, when a person makes a grammaticality judgment on a sentence in their native language they will usually have very high confidence in their answer; that is, they have conscious judgment knowledge. But this does not mean that they are aware of the underlying rules that determine whether a sentence is grammatical or not. In Dienes' terms they have no conscious structural knowledge. To measure this aspect of a person's subjective state they can be asked to indicate, in addition to confidence, whether their judgement was based on a guess, intuition, memory, or rule. Intuition means that they are confident in their answer without knowing why, which is the state assumed for native judgements about grammaticality in natural language, and assumed to be a valid measure of implicit structural knowledge (Dienes \& Scott, 2005).

Pros: Asking participants to introspect their mental states and the state of their knowledge taps on the issue of awareness directly. Also, awareness is measured concurrently with the test task, so the method does not face the retrospective problem faced by postexperiment reporting. Furthermore, participants are not required to formulate their knowledge exactly, therefore even vague, imprecise notions can be reflected in answers with slightly higher confidence. The method is therefore more sensitive than post-experiment verbal 
reports. It also provides a solution to the problem that any process is unlikely to ever be purely implicit or explicit, by filtering out the responses which were marked using explicit judgement and source knowledge.

Cons: Again, the reporting threshold. Participants may have low confidence knowledge which they do not report. The method assumes deep and honest introspection. Furthermore, asking participants to provide the source of their judgement on every decision they provide is likely to prompt them to intentionally search for rules during the test task. This may be a particular problem when using source judgements that include the "rule" category (Rebuschat et al., in preparation).

\section{Automaticity}

Method: Automaticity is an indirect measure of implicitness. It is the basis of numerous different tasks, in which participants do not fully control their performance, e.g., reaction time tasks, such as those reported in Leung \& Williams $(2011,2012)$ studies or in the process dissociation procedure (Jacoby, 1991).

Pros: Similarly to subjective measures, provides a solution to the problem that any process is unlikely to ever be purely implicit or explicit. Simply put, the presence of an implicit process can be detected if performance is different from what it would have been if it had been purely explicitly controlled. Also, the awareness of the learning target usually does not form part of the test task requirement, nor is this awareness even particularly beneficial for the task. For example, reaction time tasks - unlike grammaticality judgement tasks - can be performed without participants being aware of the existence of any regularity. It is not of any benefit in such a task to possess explicit awareness, which avoids the problem of participants intentionally constructing hypotheses along the way.

Cons: Often the task instructions are too complicated to apply in disadvantaged populations, such as amnesic patients. Also, the external validity, especially in a linguistic context, may be compromised. After all, in a natural linguistic situation people are not usually pressed for time to give responses. Therefore it is not clear whether conclusions from this method may always be extended to situations outside the lab. 


\section{Neuroimaging}

Method: Neuroimaging methods are rapidly developing. Currently available ones include event-related brain potentials (ERP), magnetoencephalography (MEG), functional magnetic resonance imaging (fMRI), and positron emission tomography (PET).

Pros: Although the methods so far have been rather crude, neuroimaging may prove more sensitive than behavioural data, or supply otherwise unobtainable data. For example, ERP studies carried out by Morgan-Short, Steinhauer, Sanz, \& Ullman (2012) revealed that among participants performing comparably on behavioural tests, some may exhibit a more native-like P600 effect, while others, a more non-native like N400. This suggests that people can accomplish the same means via different mental strategies, which would be unlikely to be possible to introspect. Also, PET studies have been reported identifying some areas of the brain being active when participants report awareness (Rauch et al., 1995). There has even been a report that some brain areas might be responsive to novelty in the absence of awareness (G. S. Berns, Cohen, \& Mintun, 1997).

Cons: The studies linking performance to awareness have been relatively scarce so far and the results may sometimes be difficult to interpret. Also, neuroimaging is an expensive technique. Furthermore, especially in the case of fMRI - it requires accepting at least one other method.

It becomes clear that each method has its useful and not useful aspects. Perhaps it is difficult to make definite conclusions about new results given the outcome of just one method. An advantage of the investigations presented in this dissertation consists of addressing semantic implicit learning via an array of different methods based on subjective measures, automaticity, and in Chapter 5 ERP neuroimaging. The way in which the experiments address the methodological issues raised here will be discussed in the relevant chapters.

\subsection{Chapter summary}

This chapter introduces the research question: does implicit learning of semantic generalisations based on content words (such as verbs or nouns) exist? In particular, is there 
semantic implicit learning of verbs' semantic preferences? The literature overview places the question in the broader context of implicit learning research, most of which has so far focused on form-level regularities. It is argued that looking at implicit learning of abstract regularities, both outside as well as within language, can bring new insights into both fields: psychology and linguistics. On the one hand, investigating the phenomenon of implicit learning in this new domain can deepen our understanding of the mechanisms that underpin it. On the other hand, prior work on implicit learning provides a rigorous methodological framework to address the acquisition of word use in second language. Finally, the pros and cons of different methodologies were discussed. 


\section{Chapter 2}

\section{Semantic implicit learning in acquisition of L2 English}

\subsection{Introduction}

Does semantic implicit learning naturally occur in a foreign language learning setting? Below is a report of the first empirical test of whether Polish learners of English indeed possess and develop semantic implicit knowledge at all. Determining the existence of such knowledge is an important step, not only in light of the controversies surrounding the existence and mechanism of implicit learning (as discussed in Chapter 1) but primarily in light of the pedagogical debate between advocates of communicative and functional teaching methods.

The communicative approach to teaching arose in the 1970s as both linguists and educators grew dissatisfied with the then predominant audio-lingual and grammar-translation methods. It became clear that even despite elaborate explicit knowledge of language, learners often felt robotic and at a loss in natural, social situations. A solution to that was the development of new classroom methods and activities with the chief focus on communication and meaning, such as role-plays, interviews, information gap-fills, games, surveys and pairwork. Margie Berns, an expert in language pedagogy and sociolinguistics wrote: "language study has to look at the use (function) of language in context, both its linguistic context (what is uttered before and after a given piece of discourse) and its social, or 
situational, context(...)" (Berns, 1984, p. 5). Of course underlying this framework is the assumption that learners will acquire the crucial aspects of language automatically.

Currently, the extreme communicative approach is not favoured any more, after succumbing to the criticism that plain "chatting" does not stimulate actual learning of the material necessary to improve competence (and confidence). Currently, anyone who has followed a CELTA (Certificate of English Language Teaching to Adults) teacher course at least within the last few years will have been taught a mixture of "focus on meaning" and "focus on form" teaching methods and encouraged to balance them and apply them for different purposes. In sum, currently the question of high importance is which aspects of language can be acquired naturally, as communicative approaches suggest, and which aspects require explicit teaching, as favoured by functional teaching methods. Implicit learning provides an ideal methodological framework within which to find this out.

One may still wonder, however, why examine implicit learning, as opposed to only incidental? After all, surely learning that leads to explicit knowledge should in principle be at least as useful as the development of unconscious knowledge? There is evidence to believe that learning and acquisition processes not only are different (Krashen, 1981), but also involve mental linguistic representations of a different type. Morgan-Short, Steinhauer, Sanz, \& Ullman (2012) showed that learned and acquired structures are associated with different neural signatures, and only learners who had undergone "implicit" (i.e. inductive and noninstructed) learning displayed native like P600 responses to syntactic violations, whereas explicitly instructed learners, whilst achieving similar levels of proficiency on behavioural tests, did not display native-like ERPs. These differences, and their implications for the trajectory of learning versus acquisition, are only now beginning to be understood, despite their profound importance for strategies of language pedagogy.

Is semantic implicit learning a real phenomenon in language acquisition? Do people acquire knowledge about the meanings of words in a foreign language? If so, what drives such learning? In the light of the discussion in Chapter 1, specifically the role of similarity between training and test stimuli, this initial experiment examines whether the knowledge of meaning - once acquired - can automatically be applied to various contexts in which a given word is used. Does it matter how similar the test case is to the contexts to which the learner has been exposed?

In short, Experiment 1 examines whether participants exposed to novel L2 words in written contexts are able to: 
- incidentally develop sensitivity to the legitimate and illegitimate usage of the words in new but similar contexts (where context is operationalised as the type of nouns with which a given verb collocates);

- extend this sensitivity to new and semantically different contexts;

- $\quad$ assess to what extent the performance is based on implicit knowledge.

\subsection{Experiment 1}

Participants: 68 university students at the University of Economics, Krakow, Poland. They were from 5 classes at the upper-intermediate/advanced level. The students in each class were divided into the experimental and control groups.

\subsubsection{Stimuli}

Four target words of English: DIMINISH, DEPLETE, TRUNCATE, MITIGATE. Chosen to specify the following conditions:

- Not have an easily identifiable counterpart in Polish. Otherwise the experiment would amount to a simple test for how many people can find the right equivalent. This was ensured by a pilot questionnaire where 7 Polish native speakers of an advanced command of English, judged the fit of the strongest Polish equivalent for each experimental stimulus and suggested other Polish words where the fit was low. The results indicated that none of the words have a single best equivalent, with mitigate having one fit for the most contexts, namely the word: lagodzić, which also means 'to ease', 'alleviate', 'smooth over' or 'commute' as in 'commute punishment'. The word mitigate does also have cognates in Polish: mitygować and mitygować się meaning 'stop someone else or oneself, respectively, from making a rash decision'. These are, however, very old fashioned expressions, possibly not even known by a number of participants, and crucially, used that way the word takes an animate patient as argument, which would not match the contexts provided in the experiment. Further 
elaboration on this issue will be mentioned in the discussion at the end. The Polish translation equivalents which could be applied in the experimental contexts are presented in Table 2.1 below.

Table 2.1. Polish equivalents of the learning targets appropriate in the sentences used in Experiment 1.

\begin{tabular}{llll}
\hline diminish & deplete & truncate & mitigate \\
\hline zmniejszać & wyniszczać & ściać & tagodzić \\
umniejszać & niszczyć & okroić & umilić \\
pomniejszać & pochtaniać & obciaćc & \\
ograniczać & wypierać & & \\
\hline
\end{tabular}

- Be highly related in meaning, so that the context serves as the crucial cue for their differentiation. Here, all the words indicate 'reducing something' or 'making something smaller/weaker'. Mitigate indicates making an unpleasant situation or its effects less serious and less difficult to bear. Out of the four words it is the only one with a positive connotation. Diminish means 'to make something smaller', and is mostly used with abstract nouns, such as 'value' or 'importance', rather than concrete nouns, such as 'food' or 'house'. Out of the four words, only this one may also be used to deliberately make someone or something appear less important than they really are. Deplete means to reduce supplies of something to a level that is too low. Unlike 'diminish', it is usually used with tangible, material components. 'Truncate' suggests reducing the length of something by cutting off a part of it.

- Be different enough from each other to find non-overlapping contexts where none of the other three words fits. This was ensured by a pilot study in which a group of 4 English native speakers were individually presented with experimental stimuli and asked to indicate whether 1) they would naturally use a proposed word in the contexts, and 2) if any of the other three words were possible there as well. 
- Be unlikely to be known by an intermediate-level L2 English learner.

Contexts for training and test items were selected from the British National Corpus and the Brigham Young Corpus of American English which met the above contextual criterion. Figure 1 presents sample training sentences for each word and Figure 2.1 presents sample test stimuli. The stimuli material is presented in Appendix A.

\subsubsection{Procedure}

\section{Training}

The experiment took place in a classroom environment. Half of a class was given full experimental training with 50 sentence contexts: 8 with each target word and 18 filler sentences. The other half of the class served as a control group and were given sets with the same sentences but with the target words paraphrased by synonyms. In the training task participants rated each sentence for how important the information was to them on a scale from 1 to 5 .

\section{Examples of the training items:}

Research shows that having strong ties to co-workers can help mitigate job related stress - a good reason to make some office buddies.

0

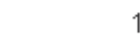

1

○
2

The economic crisis has visibly hit the university graduates who are just entering the workforce. Boston Consulting among many companies had to severely truncate the lists of accepted interns, leaving many hopeful youngsters jobless.

$0 \quad 1$

P
1

-
3

O
5 8

The public obviously has had enough of the prime minister trying to diminish the status of the president and vice versa.

0 0
1 ○
2 0
3 -
$4-5$ 0

In Nepal, 120,000 trekkers a year deplete forests by always using wood in lodges or houses for baths and cooking. Many forest dwelling animals are facing extinction as a result. 
Each target word in the training constantly collocated with nouns from one semantic domain, and so:

- mitigate - with negative psychological states

- diminish - with nouns of importance

- deplete - with natural resources

- truncate - with words denoting measurable strings of text

A full list of target word arguments used in the training as well as the test is presented in Table 2.2.

\section{Test}

The test was unexpected. Participants were not informed beforehand in order to create an incidental learning condition. Both the experimental and control groups received the same test. Stimuli were semi-randomised, with the same word never appearing twice on the same page. There were 48 sentence contexts: 12 for each word, 6 times used correctly, 6 times incorrectly. The task was to determine whether the target word, presented in capitals, was used correctly or not. Participants were also asked to indicate the source of their judgement. Figure 2.1 presents an example test sentence with the tasks. Subjective measures of awareness were modelled after Dienes \& Scott (2005). "I formulated a definition" is akin to their 'rule' category, "I remembered a similar sentence" is akin to "memory', and "I knew before what the word means" is included here to catch cases where the participants already knew the word.

The bad news did not DIMINISH her enthusiasm for the plan, she remained just as excited as she was before.

\begin{tabular}{|l|l|}
\hline \multicolumn{2}{|c|}{$\begin{array}{c}\text { Is the word } \\
\text { correct? }\end{array}$} \\
\hline YES & NO \\
\hline
\end{tabular}

$$
\begin{aligned}
& \hline \text { How did you mark your answer? } \\
& \hline \text { - by guessing } \\
& \text { - intuition } \\
& \text { - I formulated a definition and used it } \\
& \text { - I remembered a similar sentence } \\
& \text { - I knew before what this word means }
\end{aligned}
$$

Figure 2.1. Example test item. 
The correct uses were divided into two subtypes: the items where the target collocated with semantically similar arguments, and those involving extension to a new semantic domain. To illustrate, the word truncate in the training only appeared collocating with members of the category of textual strings (e.g. message, lists). Therefore 3 of the 6 correct test items presented truncate with new textual strings (not encountered in training). The remaining 3 correct items involved truncate collocating with nouns from a new semantic domain, in this case nouns of temporal duration. In these sentences, like in all sentences where it appears, truncate is the only word out of the four targets to possibly fit semantically. However, in order to correctly judge the acceptability of truncate in the new, extended contexts the participants would have to have learned something about the meaning of truncate that differentiates it from the other words. Table 2.2 illustrates the distribution of nouns in the training and test, arranged into semantic types.

The 6 incorrect stimuli were also not random, as they consisted of the target words presented with the noun type where a different target word appeared in training ( 3 times) and where a different word was used as an extension context (3 times).

\section{Post-experiment questionnaire}

The test was followed by a short questionnaire in which the students were asked 1) whether they saw the target words in the first part of the experiment (with the aim of indicating to the experimenter which group they belonged to), 2) whether they had any ideas about what these words mean, 3) to estimate how much of the training text they understood, 4) whether they were trying to figure out the meanings of the target words as they were doing the test, 5) to provide any further comments. 
Table 2.2. Words and their arguments in the well-formed sentences in training and test.

\begin{tabular}{|c|c|c|c|}
\hline & TRAINING & SAME TYPE & EXTENSION TO... \\
\hline MITIGATE & $\begin{array}{c}\text { Negative psychological } \\
\text { states: } \\
\text { Grief } \\
\text { Pain } \\
\text { Stress } \\
\text { Depression } \\
\text { Loss } \\
\text { Suffering } \\
\text { Despair } \\
\text { Agony }\end{array}$ & $\begin{array}{l}\text { Humiliation } \\
\text { Frustration } \\
\text { Fear }\end{array}$ & $\begin{array}{l}\text { Natural disasters } \\
\text { Climate change } \\
\text { Global Warming } \\
\text { Flood damage }\end{array}$ \\
\hline DIMINISH & $\begin{array}{l}\text { Importance: } \\
\text { Force } \\
\text { Input } \\
\text { Influence } \\
\text { Significance } \\
\text { Prominence } \\
\text { Greatness } \\
\text { Status } \\
\text { Role }\end{array}$ & $\begin{array}{l}\text { Accomplishment } \\
\text { Reputation } \\
\text { Importance }\end{array}$ & $\begin{array}{l}\text { Feelings: } \\
\text { Enthusiasm } \\
\text { Gratitude } \\
\text { Desire }\end{array}$ \\
\hline DEPLETE & $\begin{array}{c}\text { Natural/biological } \\
\text { resources: } \\
\\
\text { Ozone layer } \\
\text { Forests } \\
\text { Nitrogen } \\
\text { Oxygen } \\
\text { Carbohydrates } \\
\text { Natural resources } \\
\text { Water reserves } \\
\text { Essential nutrients }\end{array}$ & $\begin{array}{l}\text { White blood cells } \\
\text { Serotonin } \\
\text { Oil supply }\end{array}$ & $\begin{array}{c}\text { Financial resources: } \\
\\
\text { Money } \\
\text { Treasury } \\
\text { Savings }\end{array}$ \\
\hline TRUNCATE & $\begin{array}{c}\text { String of text (length) } \\
\text { Line } \\
\text { Message } \\
\text { Text } \\
\text { Lists } \\
\text { Sentences } \\
\text { File } \\
\text { Section } \\
\text { Website URL }\end{array}$ & $\begin{array}{l}\text { Password } \\
\text { Document } \\
\text { Manuscript }\end{array}$ & $\begin{array}{c}\text { Nouns for temporal } \\
\text { duration: } \\
\\
\text { Transmission } \\
\text { Show } \\
\text { Presentation }\end{array}$ \\
\hline
\end{tabular}




\subsubsection{Results}

Overall accuracy in the experimental group $(\mathrm{n}=35)$ was $56.1 \%, \mathrm{SD}=0.11$, whereas among the control group $(\mathrm{n}=27)$ it was $51.8 \%, \mathrm{SD}=0.06$. The difference between the groups achieved significance at $\mathrm{t}(26)=1.97 \mathrm{p}<0.05$ in a one-tailed t-test. Figure 2.2 below presents the distribution of responses across the source judgements.

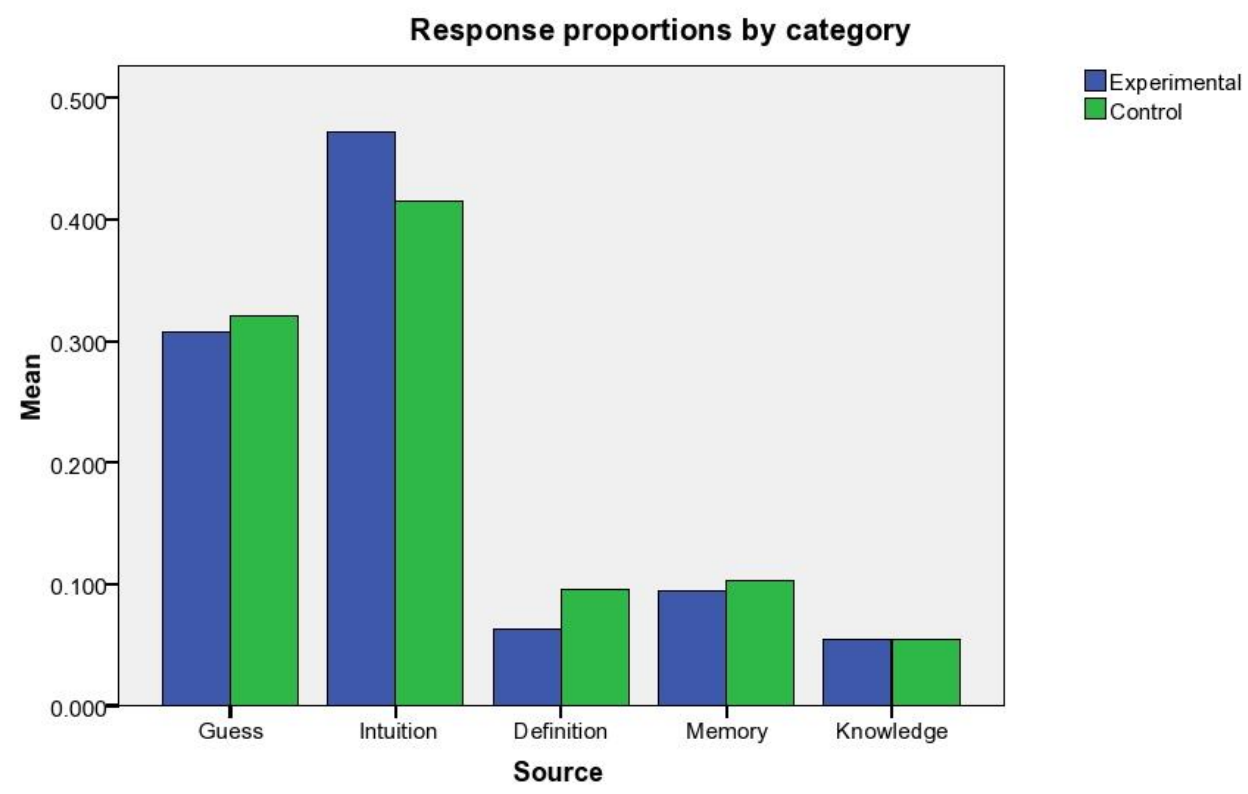

Figure 2.2. Response proportions by category.

Most participants cited 'guess' and 'intuition' as sources of their judgements. Following Dienes \& Scott (2005), such responses are presumed to reflect implicit knowledge. The following analysis therefore includes only these answers.

Truncate turned out to be the only word for which the trained group did not outperform the controls numerically. 


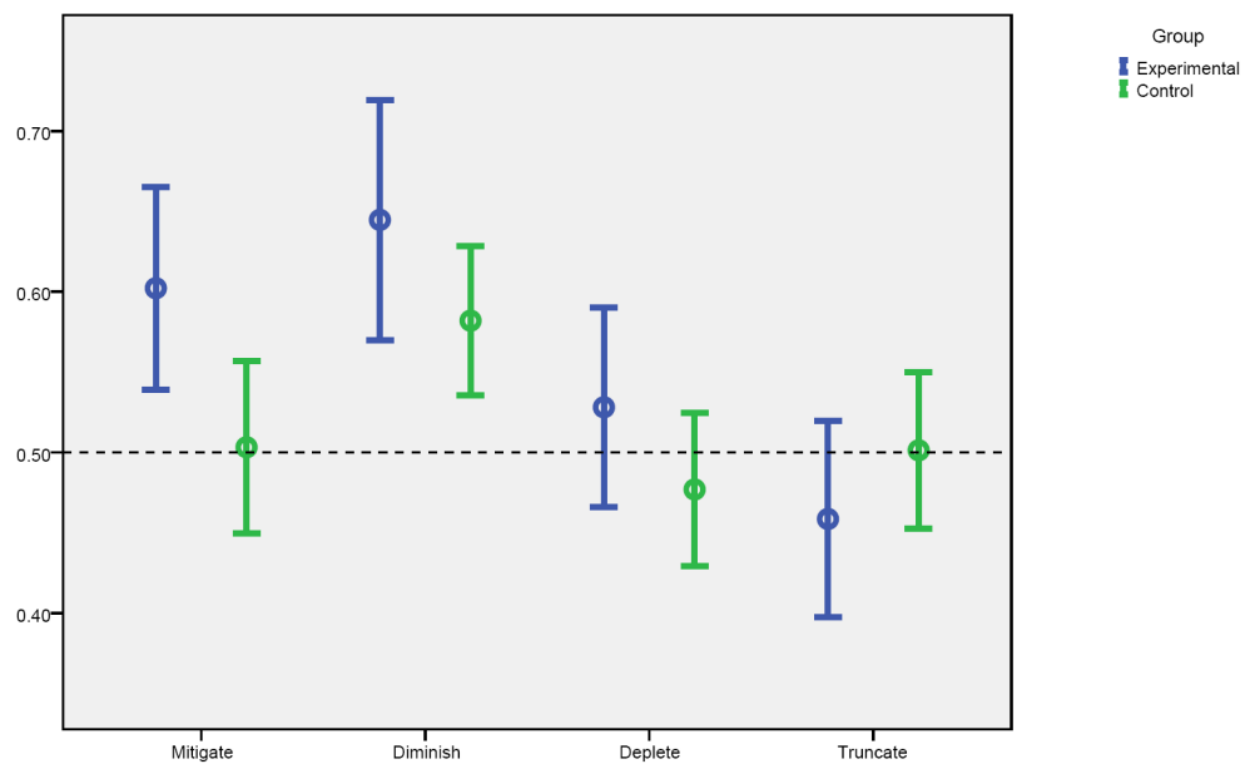

Figure. 2.3. Accuracy of the implicit source responses by word, $0.5 \mathrm{CI}$.

The word truncate turned out also to be the only word that failed to show any learning effect even on the grammatical items in the similar contexts to training. Since there are independent reasons for expecting this word to be the hardest to learn (see Discussion) it has been excluded from the following analyses.

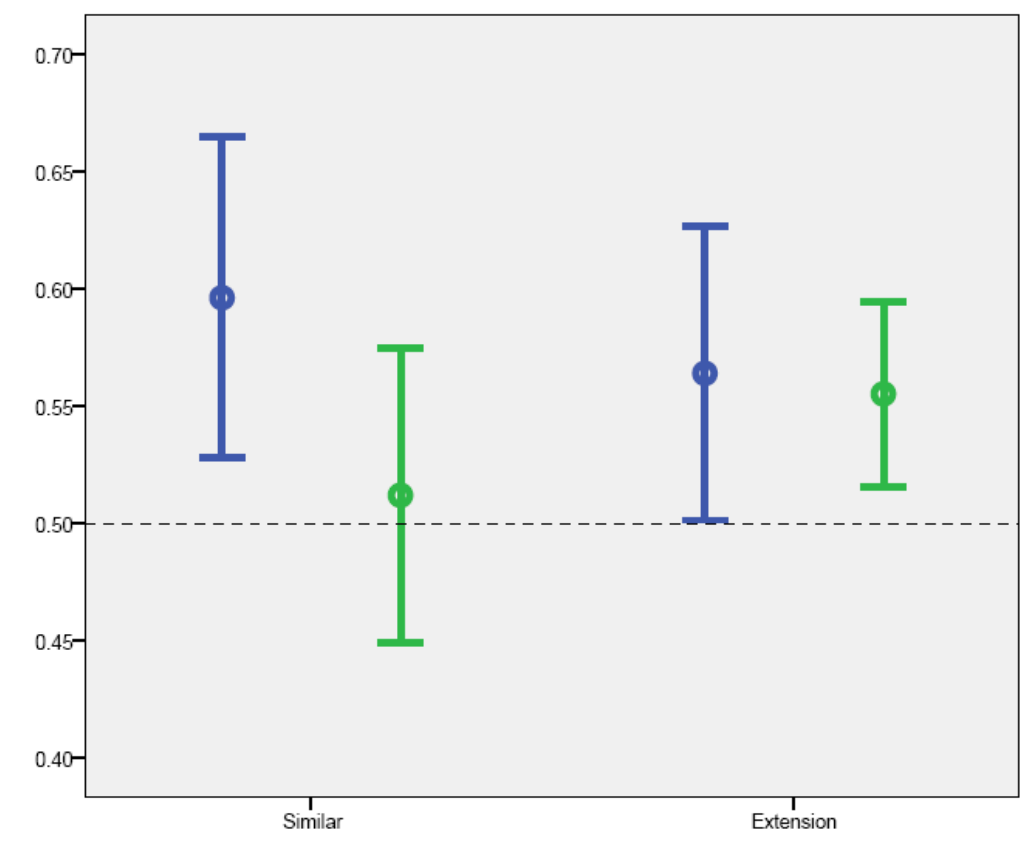

Group

Experimental

Control

Figure 2.4. Accuracy in similar and new contexts (extensions) for implicit source, $0.5 \mathrm{CI}$. 
Excluding truncate, in the similar contexts the experimental group outperformed the control group, $\mathrm{p}<0.05$ (one-tailed t-test), whereas there was no significant difference in the extension context. This suggests implicit knowledge of word usage in similar contexts only.

As mentioned above, in the test each word appeared 12 times, 6 times correctly and 6 incorrectly. Out of these 6, 3 times the word was used in similar contexts as in training and 3 times in contexts which were correct (grammatical), but semantically different to the training items (extended contexts). Figure 2.5 presents accuracy for the grammatical and ungrammatical items in similar and extended contexts for the answers marked as guesses and intuition. On these implicit judgements, planned comparisons showed that the mean accuracy of the experimental group on grammatical items appearing in similar contexts to the ones in training (70\%) was significantly different from the control groups' performance $(59.5 \%), \mathrm{p}<$ 0.05 . No differences were found in the other conditions.
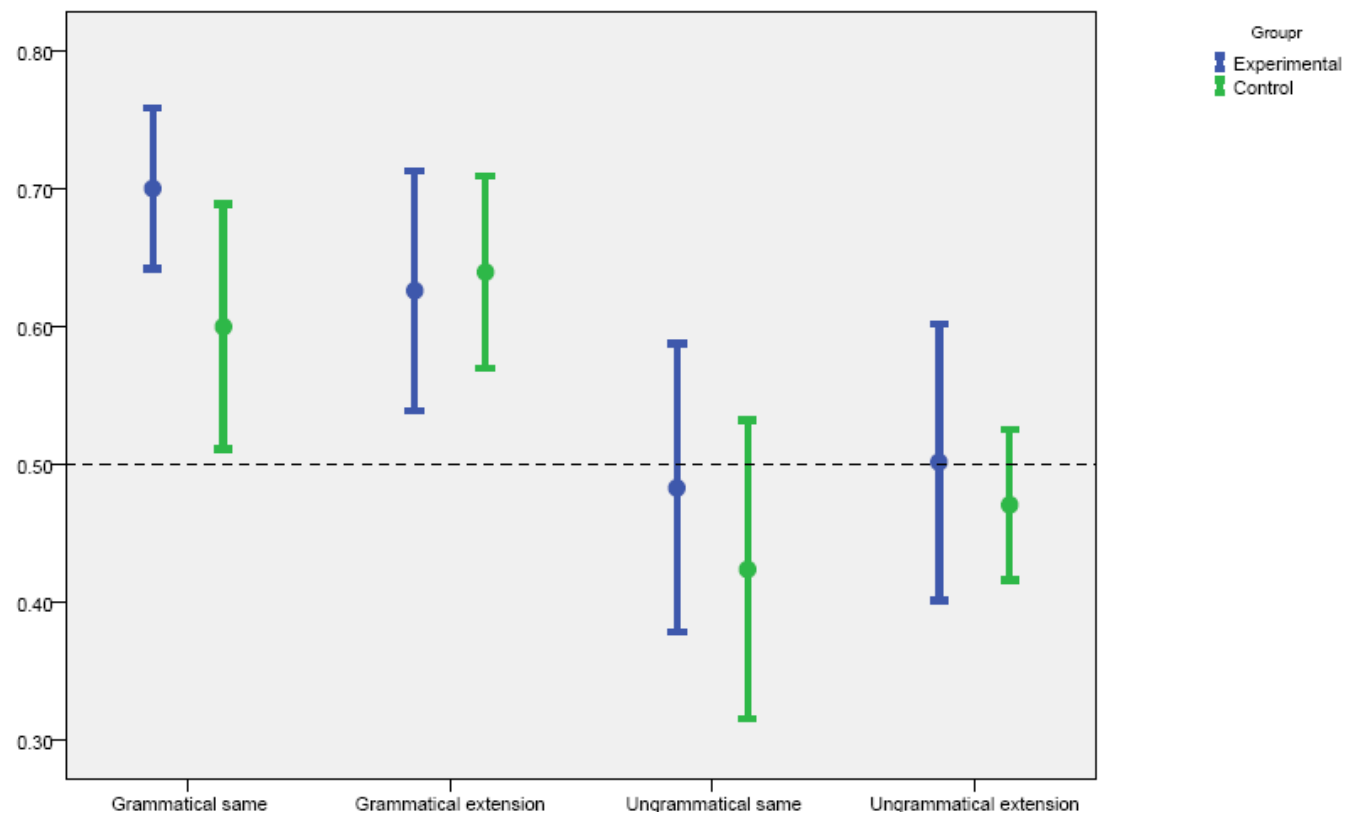

Figure 2.5. Accuracy for grammatical or ungrammatical items in similar or extended contexts for implicit source, $0.5 \mathrm{CI}$. 


\subsection{Discussion}

\section{Implicit learning}

Learners seem able incidentally to develop sensitivity for the legitimate uses of new words in novel contexts that are semantically similar to the ones to which they have been exposed. They do not appear to extend this knowledge to semantically different novel contexts, or to reject incorrect uses. Nevertheless, because their performance is so different on the two types of contexts (similar and new) they must implicitly have formed certain generalisations about the semantic environment accompanying the words. It is remarkable that an effect was obtained despite the fact that none of the words appeared with the same noun collocate more than once. This is the first demonstration of semantic implicit learning occurring on content words in language and suggests that abstract level generalisations, which have predominantly been denied to implicit learning processes, may indeed take place in language.

The fact that that the experimental participants performed better on grammatical test items similar to the training items than on completely novel ones is reminiscent of the research in implicit learning in other domains (e.g., Rebuschat, 2009, Williams \& Kuribara 2008), where similar stimuli usually attract strongest learning effects, and learning is weaker - if at all - on novel applications. The results therefore support episodic models of semantic learning, but go beyond them through demonstrating that learning takes place not only at the level of form but at the level of semantic generalisation. Future work should shed light on the extent to which this generalisation may occur and what it is driven by.

\section{Second language pedagogy}

The results presented here provide empirical support for the assumptions underlying communicative approaches to language teaching, namely that learners can develop linguistic knowledge pertaining to word use without explicit mediation. Educators should however bear in mind that such knowledge will extend only to semantically similar (albeit new) instances. When it becomes known what drives or limits the process of semantic generalisation it might be possible to make specific recommendations about the optimal learner exposure of new words in different contexts for efficient acquisition. 


\section{Role of $L 1$}

The word truncate was excluded from the analysis as it did not show any learning effect even in similar grammatical contexts. This might be attributed to the fact that it is most dissimilar in form from any other words in the L1 Polish. Although unidentified in the initial study of Polish translation equivalents for the experimental sentences, there do exist Polish words which are similar in form to the English target words: mitigate - mitygować, diminish - minimalizować, deplete - wypleniać. Truncate was therefore the only target word whose form had to be learned from scratch. The notion that pre-existing forms may have facilitated learning could also be supported by the fact that the control groups performed quite accurately on the word diminish, even on answers ascribed to an implicit source. This suggests possible availability of a concept connected to a cross-linguistically similar form, which may in turn, guide the intuitions about the use of a novel L2 word. ${ }^{9}$

\section{Indications for subsequent research}

Although the results support the hypothesis that learners indeed have implicit semantic knowledge about the meanings of words they do not explicitly know, still it is hard to say whether this knowledge was developed through the experimental training given. In particular, the fact that the control group was also above chance in their performance on grammatical items suggest that they overall indeed had some implicit knowledge. After all, the target words exist in English and it is impossible to quantify the possible exposure participants may have had to them prior to the experiment. It seems therefore advisable that experiments targeting the development of semantic knowledge should use novel word forms as targets.

Furthermore, as far as methodology is concerned, a potential problem concerns the fact that participants were given a grammaticality judgement task. They may have therefore been prompted to construct ad hoc knowledge (as discussed in section 1.8.). It is therefore better if experiments of this kind rely on methods where target knowledge is not required for the task.

\footnotetext{
${ }^{9}$ The issue of form in semantic implicit learning will be re-addressed in Chapter 3, experiments $6 \mathrm{a}$ and $6 \mathrm{~b}$.
} 


\subsection{Chapter summary and conclusion}

This first empirical chapter contains one experiment, carried out on Polish learners of English in a classroom environment. The study provides initial support to the claim that learners do implicitly learn about semantic regularities pertaining to the use of words in context. The failure to show any learning effects in new contexts which considerably differed from the training ones points to the fact that semantic similarity plays an analogous role in semantic implicit learning to that of form-similarity in implicit learning targeting form regularities. 


\section{Chapter 3}

\section{Semantic implicit learning and false memory}

\subsection{Introduction}

This chapter presents a collection of seven experiments using an improved method for addressing the development of semantic implicit knowledge. Chapter 2 concluded by suggesting that other tasks than the grammaticality judgement should ideally be applied in order to prevent participants from actively constructing relevant ideas, and also that the stimuli should include novel word forms rather than existing words, to avoid the problem of accounting for possible prior knowledge.

To this end, the method used in this series of experiments is based on an adaptation of a false-memory task. False memory is an unintentional phenomenon in which a person erroneously recalls something which did not occur. Due to its involuntary nature it can be assumed to be implicit, for which reason it lends itself particularly well as a measure of implicit learning. The awareness in the current series of experiments is determined by subjective measures combining confidence rating and source judgements (Dienes \& Scott, 2005) and additionally in a post-experiment questionnaire.

Applications of false memory tasks have already led to a number of interesting insights in psycholinguistics. A classic procedure is known as the Deese-RoedigerMcDermott (DRM) paradigm (Deese, 1959; Roediger, H. L. III, McDermott, 1995). For 
example, people erroneously report that they previously encountered a word like mug if the study phase contains words that are highly related to mug (e.g., cup, beer, bowl, coffee). An explanation for these effects is tightly linked to one of the major debates in psycholinguistics, namely whether semantic representations consist of features (e.g., (Plaut \& Shallice, 1993) or mere associations (e.g. Nelson, Bennet, \& Leibert (1997), Lund \& Burgess, (1996)). In the false-memory literature, opinions are divided into two main camps: fuzzy-trace theorists suggest that people form generalisations across items (Brainerd, Forrest, Karibian, \& Reyna, 2006), while others suggest that the key factor is the backward association strength between the critical lure (the tested target) and the words in exposure phase (Roediger, Watson, McDermott, K. B., \& Gallo, 2001). The experiments reported here address the phenomenon of semantic generalisation, which, if confirmed, might be seem to lend support to fuzzy trace and prototype models. Models of semantic knowledge are not directly addressed here at this stage, but the issue will be discussed at the end of this chapter in light of the results obtained.

More recently Cleary \& Langley (2007) used a false memory procedure to investigate the question of whether syntactic priming effects are a reflection of sentence structure abstraction or processing facilitation. They showed their participants sets of eight syntactically identical but semantically nonsensical strings of words (e.g., MECHANICAL CONSUMERS SUBMIT COLDER SONGS, EFFICIENT DREAMS WRITE BETTER UMBRELLAS). Subsequently, they presented the participants with a recognition test containing previously seen strings, and unseen ones that either corresponded (critical lures) or did not correspond (new lures) to the structure in the previously seen strings, and they were asked to rate how confident they were that they had seen the string before. An example of a critical lure was TERRITORIAL DANCERS OPERATE FUZZIER CURTAINS; an example of a new lure was THE TRANSPARENT LECTURE BECAME A PRIVATE REVERENCE. Participants indicated overall higher confidence ratings for critical than for new lures. Interestingly, the effect only arose on strings which were naturally grammatical, while no confidence rating effect was reported for ungrammatical strings (e.g., SOUR A KICK CLEAN BALLOON HARD). This suggests an influence of prior syntactic knowledge, and is evocative of research reporting top-down effects on implicit learning, e.g. Brockmole et. al.'s (2008) demonstration of contextual cueing effects for chessboard arrangements, which functioned only for advanced chess players for whom the arrangements were meaningful.

As mentioned in Chapter 1, a false memory task has also been used in the implicit learning literature to detect semantic implicit learning effects in "Past Midnight" studies 
(Bright \& Burton, 1994). Participants were first instructed to memorise the times shown on a series of 30 clock faces. Unbeknown to them, all of the times displayed fell between 6 and 12 o'clock. Then, in an unexpected test, they were asked to choose from two presented pairs of clock faces, selecting the one which they thought appeared in the previous part of the experiment. Even though in fact all of the clock faces were new, participants showed preferences for the ones displaying times between 6 and 12, even when they reported no awareness of the regularity at the end. Although this finding was initially taken as a demonstration of an abstraction of a ' 6 to 12 ' rule, a subsequent replication study (Newell \& Bright, 2002) revealed that this was not the case. Rather, participants encoded the times in a rough manner and most likely based their answers on how closely the test items resembled the ones they saw in the first part. Participants were therefore suggested to engage in general similarity matching. The fact that the effect did transfer from analogue to digital clock faces indicates that the effect could not have been driven by perceptual similarity, but was based upon the clock's 'meaning'. The authors conclude, however, that this fact does not allow them to distinguish between rule and similarity-based processing, because evidence for abstract representations is equally consistent with both forms of processing. Additionally, subjective measures of awareness applied in the test phase revealed potential explicit knowledge, which further disfavoured the hypothesis of implicit rule abstraction.

The fact that in the Newell \& Bright (2002) study participants most likely engaged in similarity matching should not be sufficient reason to abandon the idea of semantic implicit learning. The fact that they encoded the times roughly could in fact be important for allowing subsequent semantic generalisations to occur. Furthermore, testing the phenomenon in a linguistic environment may more readily lead to the development of semantic generalisations, as will be demonstrated below, since the input provides material which is naturally generalisable, perhaps more automatically so than clock faces.

\subsection{Experiment 2a}

Participants: 36 students of the University of Cambridge. 22 of them were native speakers of English, 14 were non-native speakers whose L1s included: Bulgarian $(n=1)$, Chinese $(n=3)$, 
Dutch ( $\mathrm{n}=1)$, German $(\mathrm{n}=1)$, Hindi $(\mathrm{n}=3)$, Portuguese $(\mathrm{n}=1)$, Punjabi $(\mathrm{n}=1)$, Russian $(\mathrm{n}=2)$ and Slovenian $(n=1)$. The mean age was 27 years. Twenty of them were male.

\subsubsection{Materials}

Materials were prepared based on the selectional preferences of four English words: diminish, deplete, increase and add. Both diminish and deplete can be used to convey the meaning of decrease. However as the following sentences illustrate, there are contexts in which only diminish can be applied, as they involve an abstract noun, and conversely, there are other contexts in which only deplete can be used without causing any semantic violation.

\section{Decrease:}

The fact that TV is still the most popular medium does not diminish/*deplete the significance of the radio.

The body must first deplete/*diminish carbohydrates before it can begin to burn fat.

Analogously, similar sentence contexts can be found to illustrate the contrast between the words increase and add.

\section{Increase:}

Nightingale worked tirelessly to improve public health and increase/*add the status of nurses.

Your compost pile does not require you to add $/ *^{*}$ increase minerals. It only needs moisture and heat.

A norming study was carried out in which a collection of sentences was presented to five native speakers of British English who rated the acceptability of the two alternative verbs. Only those in which the speakers unanimously indicated that only one was acceptable were included in the experimental materials. Subsequently, the English target words were substituted with four made-up words: gouble, powter, fonell and mouten. The material is presented in Appendix B. 


\subsubsection{Procedure}

Training task. Participants were told they were going to read sentences on the computer screen, for example "Nightingale worked tirelessly to improve public health and POWTER the status of nurses", and asked to perform two tasks for each sentence. First, to indicate whether the word in CAPITALS indicates roughly 'becoming less of' (-) or 'becoming more of' $(+)$. Here, POWTER means increase). They were not told which or how many of the novel words would be associated with which meaning, nor were they told that the novel words were going to be used consistently. This was in order to replicate as closely as possible a natural incidental vocabulary learning situation. However, in order to prevent the participants from forming incorrect associations, the programme would not proceed until the correct answer was given. In the training materials, there was a fixed relation between the novel verb and the semantic type of the following noun, specifically whether the noun denoted an abstract concept or a natural substance. Crucially, participants were not told of the existence of such a relation: indeed, their attention was not directed to the following noun at all. The participants' second task for each sentence was to rate the overall message as either important or unimportant in terms of their world-view.

The training comprised a total of 64 sentences. Half of these contained one of four novel nonsense words: POWTER, GOUBLE, MOUTEN, FONELL, two consistently meaning to increase something, and two meaning to decrease (the assignment of meaning depending on the version). The other half were filler sentences. Fillers had no novel words but contained an undistinguished abstract or concrete noun which appeared later in the testing phase. For these sentences participants were only asked to perform the second task - rating the importance of the message.

Each novel verb appeared eight times, collocating twice with the same noun. Therefore, throughout the training each verb occurred with four different noun collocates. There were two different sentences with POWTER the force, two with GOUBLE the authority, two with MOUTEN minerals, etc. This was intended to facilitate learning of the relevant semantic relations while avoiding repeating the same combinations too many times, which could lead to the formation of lexical chunks.

In the cases where the collocate was abstract there was always an article between the article and the noun (e.g. POWTER the strength, GOUBLE the influence). For the concrete collocates there was an article in only 2 out of a total of 32 items. Thus, the presence of the article may have provided a syntactic cue to the differentiation of the collocates, potentially 
"bootstrapping" the learning process. Note, however, that in the test phase the article was not present as a cue in the test items (see below).

The order of trials was independently randomised for each participant.

Testing phase. The test was unexpected for the participants. They were told that now they were going to see pairs of words on the screen (e.g. GOUBLE significance) and asked to perform two tasks described in the following terms:

1) Did you see these words appear together? All of the words will have appeared in the sentences, the question is, did these words appear together (i.e. more or less next to each other)?

'yes, the pairing is familiar' or 'no, the pairing is unfamiliar'.

2) How did you answer:

Was it $(g)$ guess, $(i)$ intuition, $(m)$ memory.

The instruction was followed by two practice pairs and it was explained that 'more or less next to each other' referred to the fact that all articles (in particular the) had been removed. The words were presented simultaneously, for example gouble greatness, mouten vitamins.

There were 40 test items, divided into three categories. Only 8 items involved the exact pairings of words that indeed appeared next to each other in the training part. They were classified as "Old Grammatical" pairings. The remaining 32 items were equally divided between the "New Grammatical" (well-formed) and "New Ungrammatical" pairings. The "New Grammatical" ones, although combining a verb and a noun that appeared in different sentences in the training part, still followed the semantic collocational regularity introduced initially, namely that POWTER and GOUBLE were being followed by an abstract noun, while MOUTEN and FONELL by a noun indicating a concrete substance (or the other way round for half of the participants). The "New Ungrammatical" pairings were also composed of nouns and verbs which were individually old, but this type involved a two-fold violation. Not only the combinations of words that did not appear next to each other, also the pattern of which verb appeared with what kind of noun was violated. Examples of phrases in each category, together with their corresponding training sentences, are presented in Table 3.1.

There were two versions of the test, equally divided between the participants. The nouns used to form the "New Grammatical" pairs in version 1, were included in the "New Ungrammatical" pairs in version 2, and vice versa. That is, across participants, each collocate noun appeared equally often in ungrammatical and grammatical conditions, as did each 
nonsense word. Unlike in the training, in the test phase novel verbs were presented in lower case to minimise participants' reliance on visual memory.

Table 3.1. Example test items with their corresponding training sentences. $N G=$ New Grammatical, $\mathrm{NU}=$ New Ungrammatical, $\mathrm{OG}=$ Old Grammatical.

\begin{tabular}{|c|c|c|c|}
\hline & NG & $\mathrm{NU}$ & OG \\
\hline Test & fonell proteins & powter potassium & mouten nutrients \\
\hline Training & $\begin{array}{l}\text { Avoid stimulants because } \\
\text { they FONELL serotonin } \\
\text { over time - this works the } \\
\text { same as stress does. } \\
\text { In clinical trials, peptides } \\
\text { derived from food } \\
\text { proteins have shown an } \\
\text { effect on blood pressure. }\end{array}$ & $\begin{array}{l}\text { Nightingale worked } \\
\text { tirelessly to improve } \\
\text { public health and } \\
\text { POWTER the status of } \\
\text { nurses. } \\
\text { Emergency treatment is } \\
\text { indicated if potassium is } \\
\text { very high, or if severe } \\
\text { symptoms are present. }\end{array}$ & $\begin{array}{l}\text { Start the day with a } \\
\text { stewed apple - it will } \\
\text { boost your health in a } \\
\text { number of ways. It is } \\
\text { light on your digestion } \\
\text { and will MOUTEN } \\
\text { nutrients rather than } \\
\text { emptying calories. }\end{array}$ \\
\hline
\end{tabular}

Post-test. After the testing phase participants performed a multiple choice test and were encouraged to think aloud in the presence of the experimenter. The test was used as means for eliciting knowledge from participants, rather than an additional measure of acquired knowledge. The first four questions probed whether they learned which word meant "to increase" and which "to decrease" something. The latter four probed their knowledge of which verb was meant to be followed by an abstract and which by a concrete noun. No feedback was given during the first four sentences, though the experimenter made sure that the participant was clear whether an "increase" or "decrease" verb was required in a sentence. In the latter four sentences the experimenter asked for justifications for the correct decisions 
and in the case of errors, feedback was given with further probing questions. Only those participants who remained oblivious of the regularity until the end of post-test were classified as "unaware". The entire procedure took about 45 minutes.

Prediction. If implicit learning takes place then the erroneous endorsement rate for the New Grammatical items will be higher than the New Ungrammatical ones for participants who show no evidence of awareness of the system in the post-test.

\subsubsection{Results}

In the post-experiment debriefing, only 2 out of 36 participants expressed awareness of the regularity from the training part. A further 7 participants made potentially crucial observations suspecting that the distinction between words related to either conceptual or physical adding/removing, or reported that having a chance to compare example sentences directly allowed them to figure out the system. Even though they claimed that these observations occurred to them only during the post-test, and could in fact themselves be an effect of implicit learning, they were assigned to the 'aware' group in the interest of maintaining a conservative measure of awareness. The remaining 27 participants were classified as 'unaware'.

The following data analysis is performed on means corrected for the small number of responses (unless otherwise stated). The correction corresponds to a Bayesian prior of chance performance worth one observation (Kiyokawa, Dienes, Tanaka, Yamada, \& Crowe, 2012), and was calculated as (number of correct responses +0.5$) /($ total number of responses +1$)$.

The analyses concentrated on the (erroneous) endorsement rates in the NG and NU conditions since the same items were used in each condition (rotated around participant groups). The (correct) endorsement rates in the OG condition are also shown for comparison and analysed separately. An analysis of variance (ANOVA) was performed on the endorsement rates from the NG and NU conditions in which Condition (NG or NU) was a within-subjects factor and participant group a between subjects factor. Weighted means are shown to correct for imbalances in the numbers of participants on each list after separation into aware and unaware groups. The mean endorsement rates for the aware and unaware participants are shown in Table 3.2. An ANOVA on these data showed that the aware group erroneously endorsed as familiar significantly more word pairs which followed the 
selectional preference pattern (New Grammatical, NG) than those that did not (New Ungrammatical, NU), $F(1,8)=6.08, p<0.05, \eta^{2}=0.43$. Accuracy for the Old Grammatical (OG) items was very good, and a single sample t-test showed that it was significantly above chance, $\mathrm{t}(9)=3.52, \mathrm{p}<0.01$.

Crucially, the 26 unaware participants showed a similar pattern in their responses. An ANOVA showed that there was a significant difference between the NG and NU conditions, $F(1,24)=9.75, p<0.01, \eta^{2}=0.29$. Memory for the OG items was equivalent to the aware group, and significantly above chance, $\mathrm{t}(25)=6.61, \mathrm{p}<0.001$.

Table 3.2. Mean endorsement rates (standard error in parentheses)

\begin{tabular}{|l|l|l|l|}
\hline & NG & NU & OG \\
\hline Aware $(\mathrm{n}=10)$ & $.51(.051)$ & $.39(.053)$ & $.70(.057)$ \\
\hline Unaware $(\mathrm{n}=26)$ & $.54(.034)$ & $.43(.040)$ & $.70(.030)$ \\
\hline
\end{tabular}

NG $=$ New Grammatical, NU = New Ungrammatical, $\mathrm{OG}=$ Old Grammatical

\section{Analysis by noun-types}

Since it is the unaware group's performance that provides evidence for semantic implicit learning, the following analysis predominantly concerns that group. First, the unaware group's data were analysed to check for any differences between the test stimuli with abstract and concrete nouns. For example, the New Grammatical item gouble value was compared with the New Ungrammatical item mouten value, or fonell hydrogen (NG) was compared with gouble hydrogen (NU). That is, the effect of grammaticality on decisions to test items containing abstract versus concrete collocates was analysed. The results are shown in Figure 3.1 . 


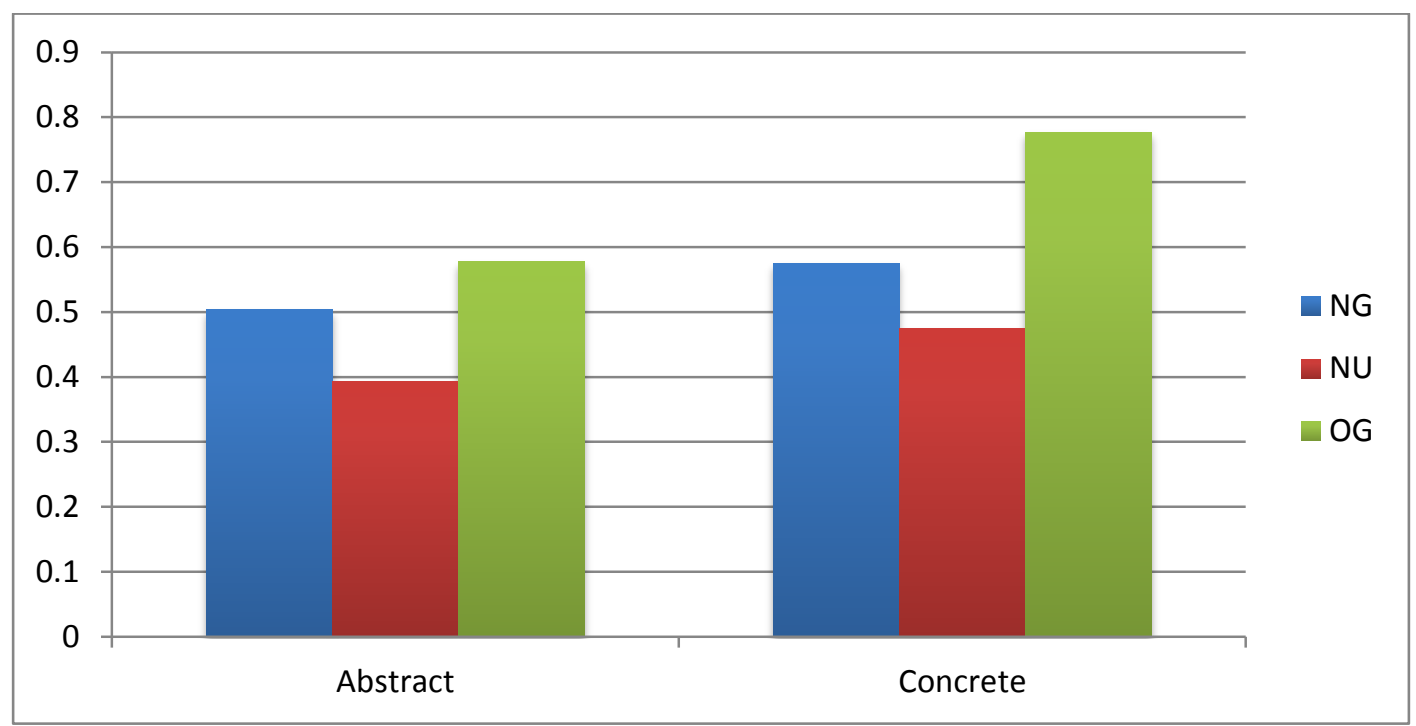

Figure 3.1. Mean endorsement rates for pairs with abstract and concrete nouns among the unaware participants.

An analysis of variance was performed on the mean endorsement rates in the NG and NU conditions and noun type as within-subjects factors, and list as a between-subjects factor. There was a main effect of grammaticality $\mathrm{F}(1,24)=9.75, \mathrm{p}<0.01, \eta^{2}=0.29$ and also of noun type $\mathrm{F}(1,24)=4.35, \mathrm{p}<0.05, \eta^{2}=0.15$, but no interaction between noun type and grammaticality, $\mathrm{F}<1.0$. An analysis of the $\mathrm{OG}$ condition also revealed a main effect of noun type $\mathrm{F}(1,24)=11.79, \mathrm{p}<0.01, \eta^{2}=0.33$.

\section{Analysis of source judgements}

For the unaware participants, the source proportions in each category of the stimuli are shown in Table 3.3. The different source attributions are evenly distributed over the three conditions. 
Table 3.3. Source proportions

\begin{tabular}{|l|l|l|l|}
\hline \multicolumn{2}{|l|}{ Source proportions } & & \\
\hline & NG & NU & OG \\
\hline guess & 0.389 & 0.389 & 0.317 \\
\hline intuition & 0.325 & 0.349 & 0.308 \\
\hline memory & 0.286 & 0.262 & 0.375 \\
\hline
\end{tabular}

Figure 3.2.shows the endorsement rates by source. The critical difference between the NG and NU conditions remains reflected within the categories of 'intuition' $(\mathrm{F}(1,21)=9.88, \mathrm{p}<$ $\left.0.01, \eta^{2}=0.32\right)$ and 'memory' $\left(\mathrm{F}(1,19)=6.61, \mathrm{p}<0.05, \eta^{2}=0.26\right)$. The effect of grammaticality on intuition responses shows that when participants only have a vague feeling of having seen the word pair before, their responses are still affected by whether the pair respects the selectional preference pattern. The well-formedness effect is stronger in the memory category, where participants actually are relatively confident that they have experienced the word pair before, and not present in the guess category. Such a pattern, in which stronger effects arise for the more confident responses, is typical for false-memory experiments.

According to the original logic of the source judgement method (Dienes \& Scott 2005) it may seem that the failure to meet the "Guessing Criterion" (above-chance responding when guessing) counts against the claim that the experiment taps unconscious knowledge of the semantic collocational pattern. However, it must be emphasised that the guessing criterion applies to tasks in which participants are required to give their opinions of acceptability. Then their performance on the task is crucially dependant on their knowledge of the system, which provides the relevant structural knowledge. This is not the case in the present experiment, as participants are only asked whether they remember seeing an item, not whether it conforms to a rule they might have observed. In fact, they do not need to presuppose the existence of any systematic regularity to perform the test. The relevant structural knowledge in this case is, simply, memory for word combinations. 


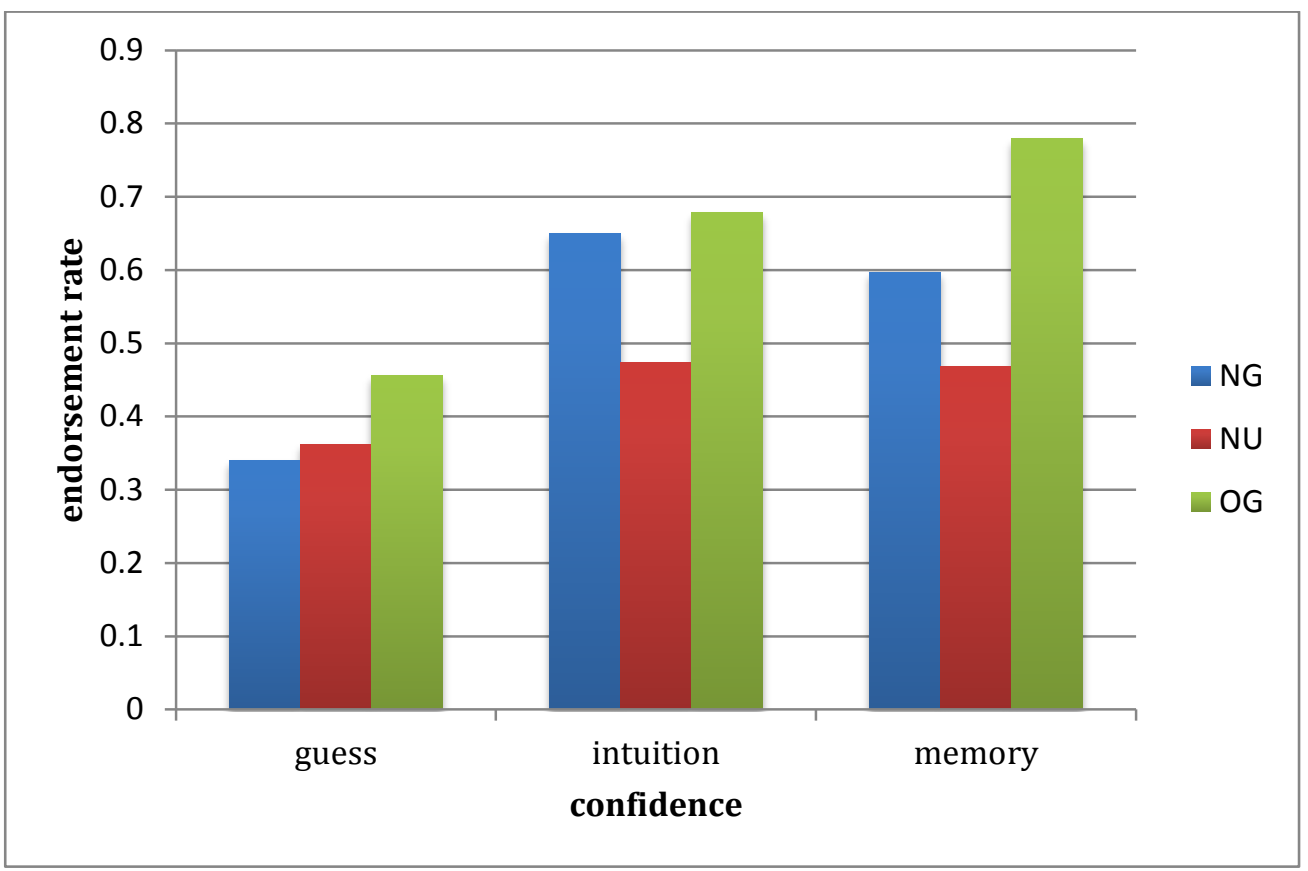

Figure 3.2. Mean endorsement rates by source among the unaware participants

Although knowledge of the semantic regularity is strictly irrelevant to the familiarity judgment task, this does not preclude participants from using such knowledge strategically. This would be particularly evident in the case of New Ungrammatical items because the failure to conform to the regularity would immediately indicate that the pair had not been seen before. A participant who was using this logic would be expected to be relatively confident when rejecting New Ungrammatical items. Judging a New Grammatical item would be harder because it conforms to the regularity and so rejections would be made with less confidence.

The participants in this experiment were not asked to indicate the confidence in their decisions, instead they indicated whether they had guessed, used intuition, or relied on memory ${ }^{10}$. In the case of endorsements it is plausible to regard these source categories as reflecting increasing subjective familiarity of the test item. This can be quantified by assigning 'guess', 'intuition', and 'memory' the numerical values 1, 2, and 3 respectively. In the context of grammaticality judgements in an artificial grammar learning experiment Scott \& Dienes (2008) show that familiarity judgements and confidence judgments are correlated (although it is possible to find variations in familiarity that are not reflected in confidence).

\footnotetext{
${ }^{10}$ This method of analysis was only arrived at after Experiment 3, at which point it was realised that for a familiarity judgment task it is more useful to elicit confidence than source judgments
} 
The scale of increasing familiarity can therefore be interpreted roughly as a confidence scale. In the case of rejections, however, the situation is less straightforward. In particular, if a person rejects a New Ungrammatical item on the basis of explicit rule knowledge, which of the source options would they be likely to select? It seems reasonable to assume that they would opt for 'memory' because they would essentially remember not having received the item, but this would be on the basis of an inference based on rule knowledge, and not on low familiarity as such. If they were less sure of the rule they might opt for 'intuition', but they would be unlikely to opt for 'guess'. In contrast, when rejecting an NG item they would be expected to experience more confusion, and would be more likely to opt for 'guess' or 'intuition'. In the case of rejections, therefore, the source categories cannot be straightforwardly aligned with familiarity (and thereby with confidence), but it is still predicted that the numerical conversion of the scale will lead to higher mean values for rejecting NU than NG. For convenience we shall refer to these values as "confidence" (note that in Experiments 4 to 6 source judgments were abandoned in favour of confidence judgments and very similar results were obtained). Figure 3.3 shows the mean "confidence" for rejections and endorsements in the three conditions.
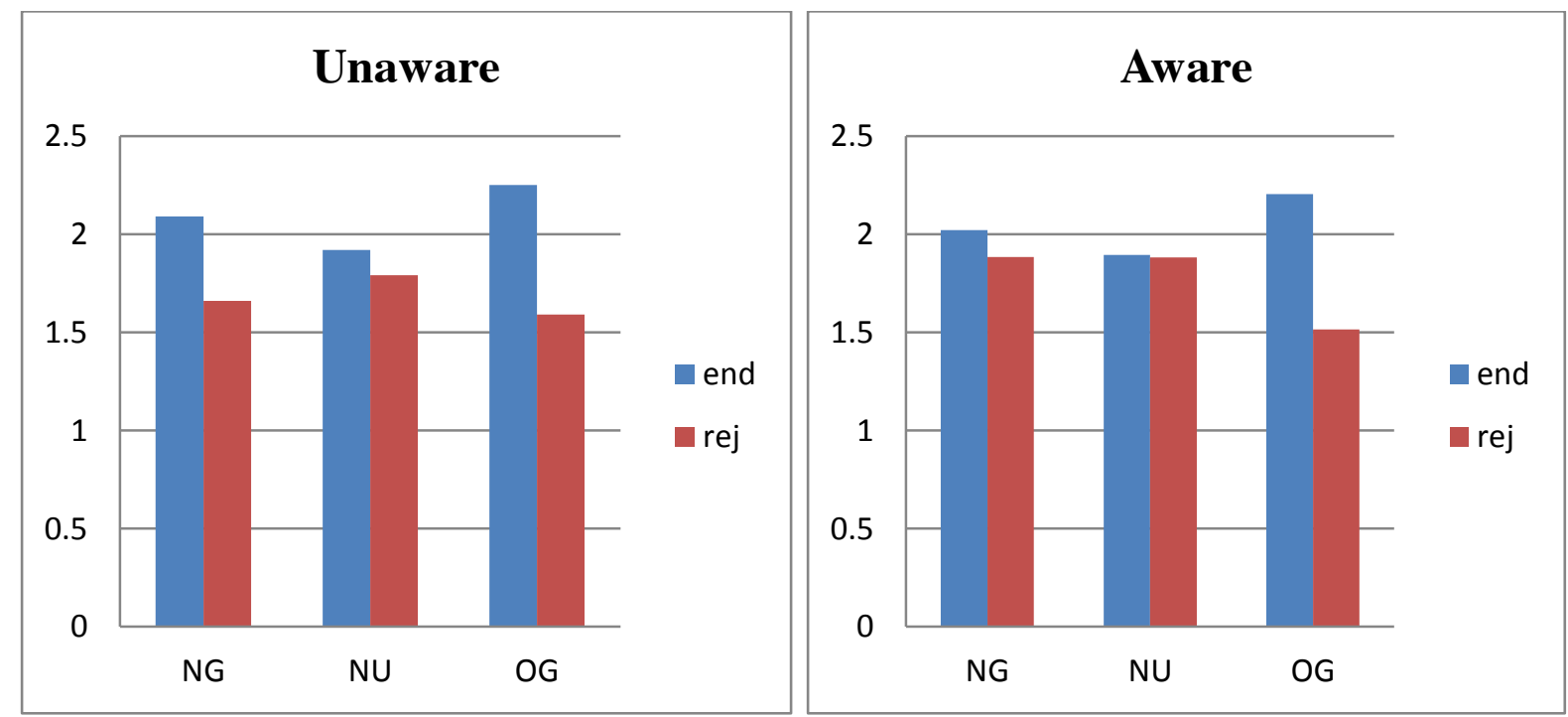

Figure 3.3. Mean confidence for endorsements and rejections. Experiment 1, a) unaware, b) aware participants. 
An analysis of variance was performed on the unaware participants' data on the mean confidence in the NG and NU conditions with grammaticality and endorsement/rejection as within-subjects factors, and list as a between-subjects factor. There was a main effect of endorsement/rejection, $F(1,24)=5.62, p<0.05, \eta^{2}=0.19$, and an interaction between endorsement/rejection and grammaticality, $F(1,24)=8.83, p<0.01, \eta^{2}=0.27$. Further analysis revealed that the interaction arises because of significantly greater confidence for endorsing $\mathrm{NG}$ than $\mathrm{NU}, \mathrm{F}(1,24)=5.24, \mathrm{p}<0.05, \eta^{2}=0.18$, and numerically greater confidence in rejecting NU than NG, an effect that approached significance, $F(1,24)=3.69$, $p$ $=0.07, \eta^{2}=0.13$. A similar analysis on the data from the aware participants revealed no significant main effects or interactions. In both groups, confidence in the OG condition was significantly higher for endorsements than rejections (unaware: $F(1,20)=18.27, p<0.001$, $\eta^{2}=0.48$, aware: $\left.\mathrm{F}(1,7)=30.98, \mathrm{p}<0.01, \eta^{2}=0.82\right)$.

In terms of the logic of the "Chan difference" as a measure of awareness (Dienes, 2008) the higher confidence for endorsing NG than NU means that participants have a greater feeling of conscious remembering for grammatical pairs than ungrammatical ones, even though both types of new pairs have not been seen before. Note, however, that endorsements are errors for NG and correct responses for OG. The effect for new pairs therefore arguably reflects the influence of grammaticality on confidence judgements. Cleary \& Langley (2007) showed a similar effect of grammaticality on confidence judgements in a false memory paradigm which they attribute to implicit memory of syntactic structure. We cannot tell, though, at this stage whether this reflects conscious or unconscious knowledge of the selectional preference rule.

Crucially though, as argued above, if conscious knowledge of the rule were affecting responses it would have its greatest effect on increasing confidence of correct rejections of NU items as compared to NG ones. Although here the difference in confidence only approached significance, there is some suggestion of contamination from conscious knowledge.

Strategic use of explicit knowledge would result in a positive correlation between the learning effect (measured as the difference in endorsement between NG and NU items) and the confidence pattern of conscious knowledge (higher confidence rejecting NU than NG). Scatter plots of the data in the unaware and the aware groups are presented in Figure 3.4. 

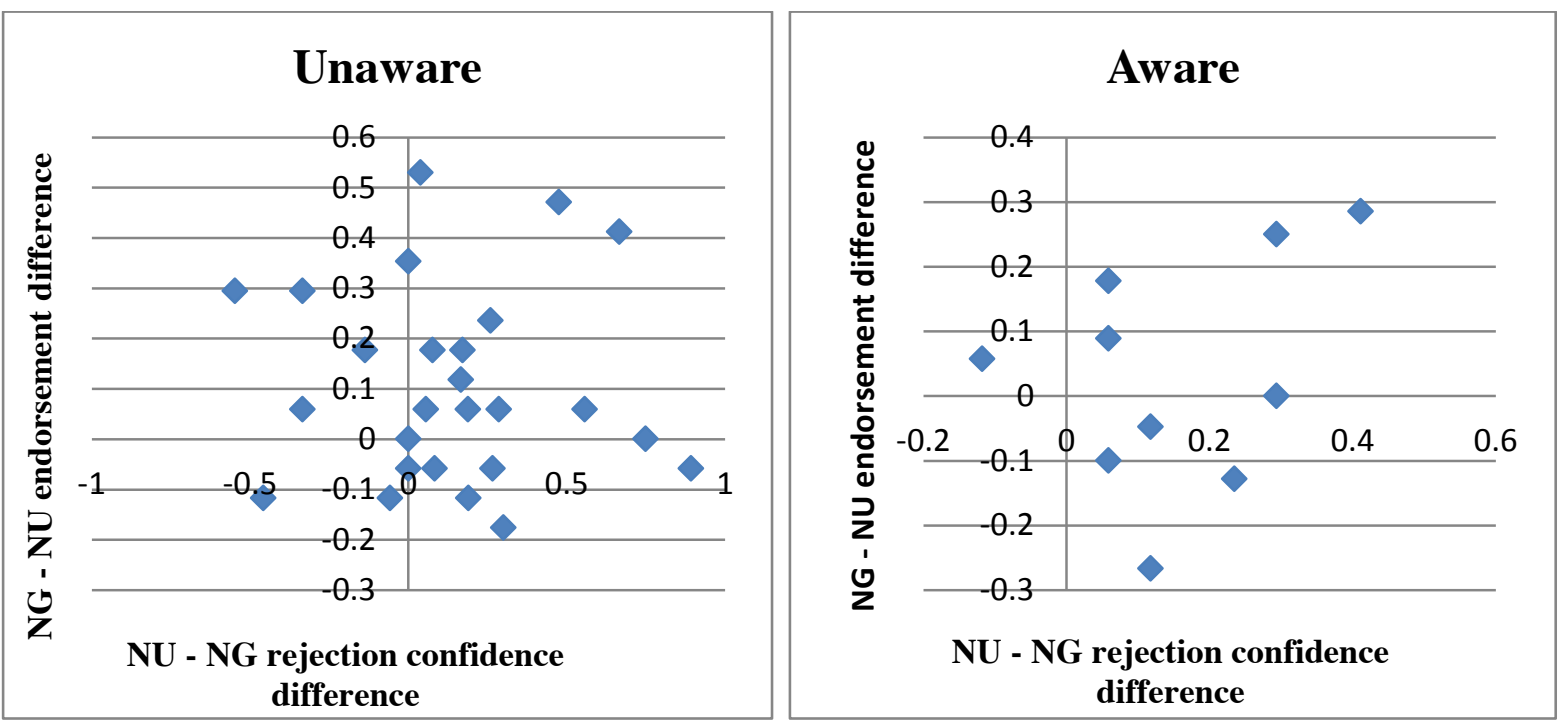

Figure 3.4. Confidence difference for NU-NG rejections and learning effect (NG-NU endorsement difference), for a) unaware, b) aware groups.

There was no correlation in the unaware group $(\mathrm{r}(25)=-0.04)$. However, as Figure 3.4a shows, there were two participants for whom a large confidence difference was associated with a large learning effect. It is possible that these participants were strategically using conscious knowledge. Nevertheless, with these participants removed from the learning effect was still significant, $F(1,22)=6.72, p<0.05, \eta^{2}=0.23$. A mild positive, but not significant, correlation was detected among the aware participants $(\mathrm{r}(7)=0.32, \mathrm{p}=0.37)$. It is worth noting, that the two aware participants for whom a large confidence difference was associated with a large learning effect (the two points with an NG-NU endorsement difference above 0.2 ) were the two participants who reported full awareness of the selectional regularity. It is arguably through them that the trend emerges.

\subsubsection{Discussion}

The experiment provides evidence in support of the hypothesis that people implicitly form semantic generalisations about the meaning of potential noun-collocates of a novel verb: in other words they implicitly learn the verb's selectional preference. The learning was assessed through an indirect measure of awareness involving participants' subjective confidence and source knowledge judgements. 
The analysis of noun-types revealed that participants in general endorsed fewer items with abstract nouns than with concrete ones. This situation could have been caused by the fact that in training between novel verbs and their following abstract nouns the definite article "the" was almost always present. Its absence in the test items could result in some feeling of ungrammaticality, as compared to concrete nouns which were not preceded by an article either in training or test. These items could be argued to have exhibited a better syntagmatic match between the training and test phases. Experiment $2 \mathrm{~b}$ addresses this concern by adding the definite article to the test items involving abstract nouns.

\subsection{Experiment 2b}

Participants: 34 students of the University of Cambridge and the University of London. 19 of them were native speakers of English, 15 were non-native speakers whose L1s comprised: Polish ( $n=6)$, German ( $n=2)$, Chinese $(n=1)$, Czech $(n=1)$, Dutch $(n=1)$, Hindi $(n=1)$, Japanese $(n=1)$, Macedonian $(n=1)$ and Portuguese $(n=1)$. The mean age was 25 years. Nineteen of them were male.

\subsubsection{Procedure}

Materials and procedure for the training part and post-test were identical to Experiment 2a. However, during the test phase, an article was included in the presentation of those test items which involved an abstract word. Well-formed pairings, for example: GOUBLE the greatness, MOUTEN vitamins, preserved the article the after GOUBLE and POWTER (for half the participants; and after MOUTEN and FONELL for those who did a rotated version), while the other two words, correctly, were not followed by the article. Conversely, the ungrammatical items, for example POWTER enzymes, not only contained a semantic violation (collocating with a concrete rather than an abstract word), but also a syntagmatic violation, since previously POWTER was always directly followed by the definite article. Similarly, phrases such as MOUTEN the value contained both a semantic violation and a syntagmatic one, as in the training MOUTEN was never followed by an abstract word or by the definite article. 
This time there were four versions of the test, equally divided between the participants. As in Experiment 2a, the nouns used to form the "New Grammatical" pairs in version 1 were included in the "New Ungrammatical" pairs in version 2, and vice versa. Furthermore, versions 3 and 4 were analogous to 1 and 2, but the novel verbs were ascribed opposite meanings and selectional preferences, e.g. gouble in version 1 selected for abstract nouns and meant "decrease", while in version 3 it selected for concrete nouns and meant "decrease". The material is presented in Appendix C.

\subsubsection{Results}

In the post-experiment debriefing 4 participants reported awareness of the semantic regularity, one of whom immediately denied using it strategically during the test. A further 6 deciphered it during the post-test. Again, to assure a conservative measure of awareness, both groups were classed as 'aware', despite the fact that awareness among the latter 6 people could itself be a result of the phenomenon of implicit learning which we are addressing.

Similarly to Experiment 2a, the analysis was done with a correction for small numbers of responses, calculated as (number of correct responses +0.5$) /($ total number of responses +1 ). Table 3.4. shows the corrected endorsement rates for the two groups of participants. The aware group failed to show a significant difference between the NG and NU conditions, $\mathrm{F}<$ 1.0, whilst their responses to OG items were significantly above chance, $t(9)=7.58, p<$ 0.001 . On the other hand, the difference between the NG and NU conditions was significant for the unaware participants, $F(1,20)=4.82, \mathrm{p}<0.05, \eta^{2}=0.19$, and their responses to the OG items were significantly above chance, $\mathrm{t}(23)=9.5, \mathrm{p}<0.001$. It is worth pointing out that the failure to find an effect for the aware participants may not be conclusive, since the raw effect size is the same for the two groups. 
Table 3.4. Mean endorsement rates in Experiment $2 b$ (standard error in parentheses).

\begin{tabular}{|l|l|l|l|}
\hline & NG & NU & OG \\
\hline Aware $(\mathrm{n}=10)$ & $.46(.070)$ & $.39(.058)$ & $.76(.034)$ \\
\hline Unaware $(\mathrm{n}=24)$ & $.53(.034)$ & $.47(.033)$ & $.79(.030)$ \\
\hline
\end{tabular}

$\mathrm{NG}=$ New Grammatical, NU = New Ungrammatical, $\mathrm{OG}=$ Old Grammatical

\section{Analysis of noun types}

An analysis by noun type (abstract or concrete) was carried out as for Experiment 2a. The results are shown in Figure 3.5.

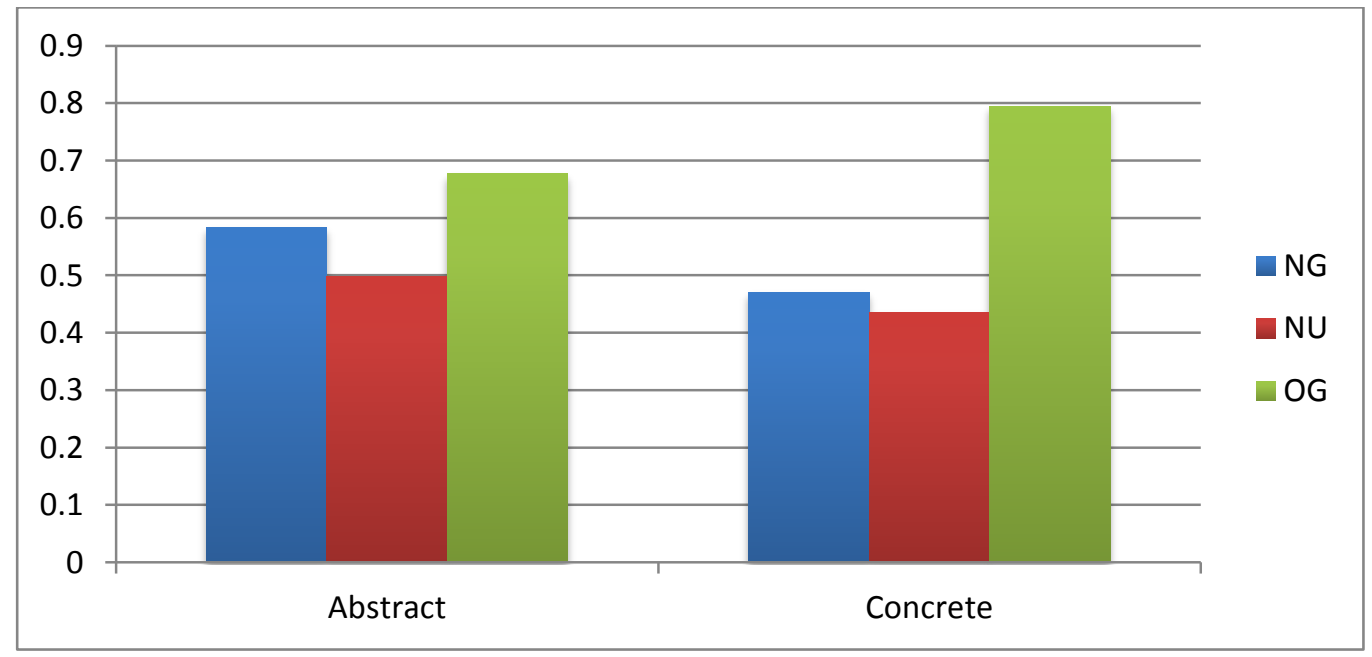

Figure 3.5. Endorsement rates for abstract and concrete collocates among the unaware participants.

An analysis of variance was performed on the mean endorsement rates in the NG and NU conditions with grammaticality and noun type as within-subjects factors, and list as a between-subjects factor. There was a main effect of collocate type, $F(1,20)=4.94, p<0.05$, $\eta^{2}=0.12$, abstract collocates being endorsed more highly than concrete ones. There was a main effect of grammaticality $\mathrm{F}(1,20)=4.82 \mathrm{p}<0.05, \eta^{2}=0.19$, but no interaction between collocate type and grammaticality, $F=1.19$. Numerically, though, the effect of grammaticality was larger for the items with an abstract collocate. Finally, for the OG 
condition, endorsement rates were higher for the concrete items than the abstract, $\mathrm{F}(1,23=$ 5.24, $\mathrm{p}<0.05, \eta^{2}=0.19$. Thus there is no general trend for the abstract collocate items to be endorsed more highly. Rather it appears that it is specifically the abstract collocate items in the NG condition which are endorsed relatively highly amongst the New conditions.

\section{Comparison between Experiments $2 a$ and $2 b$}

In order to assess the impact of the addition of the articles to the test items containing an abstract noun in Experiment $2 \mathrm{~b}$ an ANOVA was conducted to compare the results with those from Experiment 2a. In this analysis Experiment and presentation list were between-subjects factors, and condition (NG or NU) and noun type (abstract or concrete) were the withinsubjects factors. There was a highly significant main effect of Condition, $F(1,46)=14.72$, $p<$ $0.001, \eta^{2}=0.24$. The only other significant effect was an interaction between experiment and noun type, $\mathrm{F}(1,46)=8.59, \mathrm{p}<0.01, \eta^{2}=0.16$. Whilst in Experiment $2 \mathrm{a}$ test items with abstract nouns were endorsed at a lower rate than those with concrete nouns $(0.45$ and 0.52 respectively), in Experiment $2 \mathrm{~b}$ they were endorsed at a higher rate $(0.54$ and 0.45 respectively). This effect will be discussed below in Section 3.3.3. Importantly, there was no interaction between experiment and condition, $\mathrm{F}<1.0$, indicating that the size of the grammaticality effect was not affected by the inclusion of articles in test items with abstract nouns. The grammaticality effect was robust over this manipulation.

\section{Analysis of source judgements}

As in Experiment 1a, source attributions were fairly evenly distributed over the different categories, as shown in Table 3.5. 
Table 3.5. Source proportions for unaware participants.

\begin{tabular}{|l|l|l|l|}
\hline \multicolumn{2}{|l|}{ Source proportions } & & \\
\hline & NG & NU & OG \\
\hline guess & 0.326 & 0.356 & 0.266 \\
\hline intuition & 0.329 & 0.334 & 0.315 \\
\hline memory & 0.345 & 0.310 & 0.418 \\
\hline
\end{tabular}

Figure 3.6 shows the endorsement rates broken down by source. The critical difference between the NG and NU endorsement remained reflected in judgements attributed to memory $F(1,20)=6.18, p<0.05, \eta^{2}=0.24$. The effect for guess responses approached significance, $\mathrm{F}(1,20)=4.08, \mathrm{p}=0.06, \eta^{2}=0.17$, while that for intuition did not, $\mathrm{F}<1$.

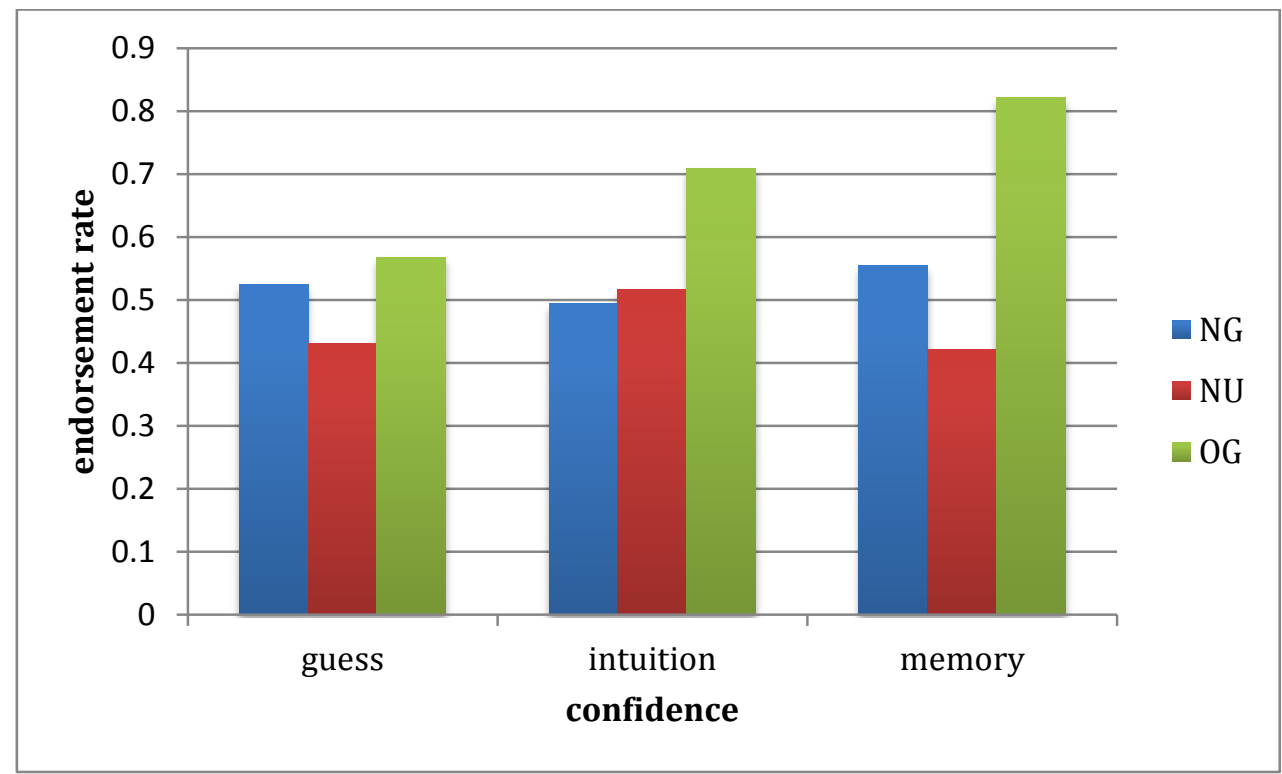

Figure 3.6. Mean endorsement rates by source among the unaware participants.

Again, it was hypothesised that conscious knowledge of the crucial semantic regularity would potentially be reflected by higher confidence in rejecting a new ungrammatical item than a new grammatical one. As in Experiment 2a, the source judgements were treated as a scale of confidence, and mean values for endorsements and rejections were calculated for the 3 conditions. The results are shown in Figure 3.7. In this case for the unaware group there were 
no significant differences in the level of confidence in endorsements and rejections in the New Grammatical and Ungrammatical conditions, $\mathrm{F}<1.0$, and likewise for the aware group, $\mathrm{F}(1,6)=1.32$. This suggests that conscious knowledge of the semantic regularity was not affecting the decision process in either group. Finally, in both groups, confidence in the OG condition was significantly higher for endorsements than rejections, (unaware: $F(1,19)=$ 6.12, $\mathrm{p}<0.05, \eta^{2}=0.24$, aware: $\left.F(1,9)=45.6, p<0.001, \eta^{2}=0.83\right)$ suggesting, as in Experiment 2a, that recognition of OG items was accompanied by conscious recognition of the word pairs.
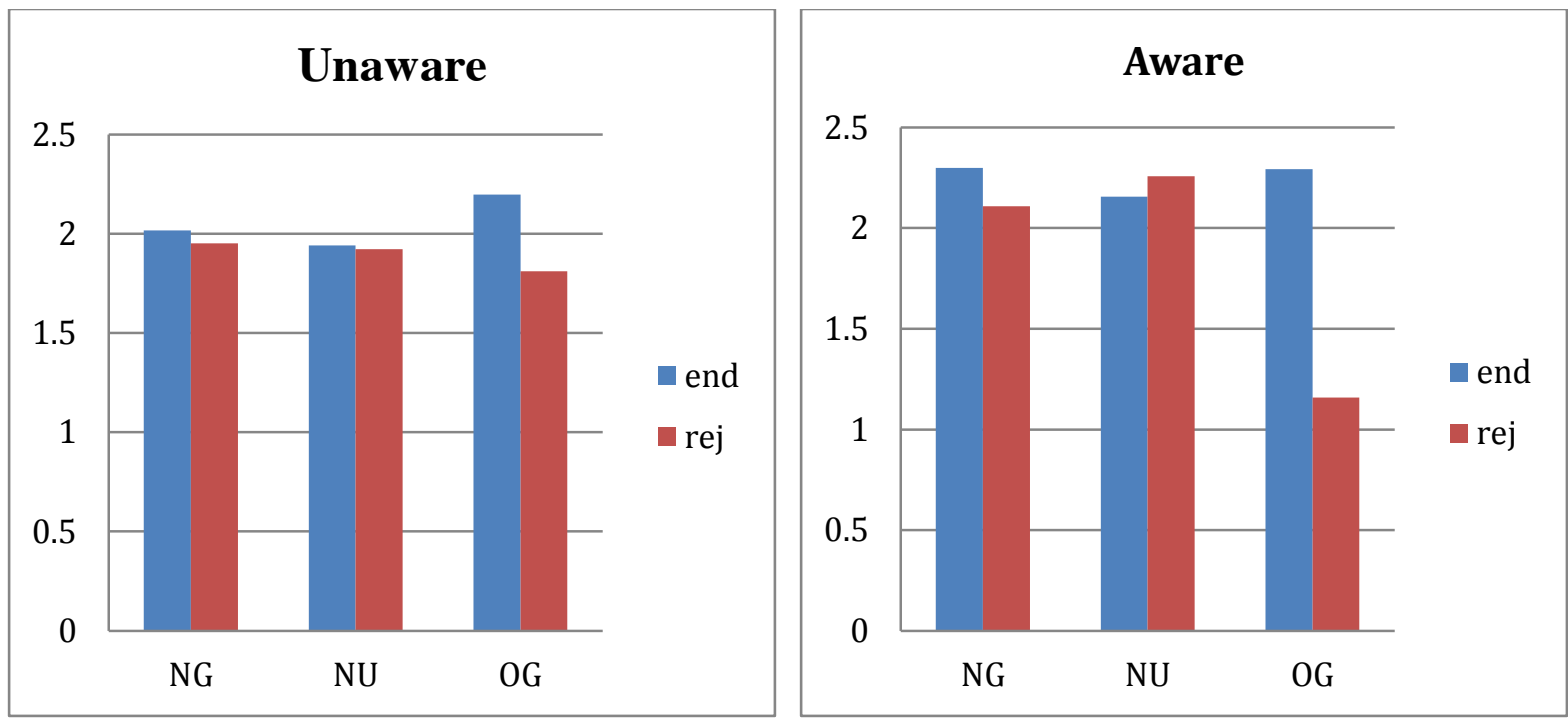

Figure 3.7. Mean confidence for endorsements and rejections for a) unaware, b) aware participants.

A finer-grained analysis looked at the correlation between the false-memory effect (the difference in endorsement between NG and NU items) and the difference between confidence rejecting NU and NG items. Scatter plots for both the unaware and the aware groups are presented in Figure 3.8. Similarly to Experiment 1a, there was no correlation in the unaware group, $r(22)=-0.05$. However, as the scatter plot in Figure 3.8. shows, there is an outlier in the top left quadrant. This data point comes from a participant with only one rejection in the NG condition. With this point removed the correlation is mildly positive, but still not significant, $\mathrm{r}(21)=0.29, \mathrm{p}>0.1$. Neither is there a correlation for the aware group, $\mathrm{r}(8)=$ 0.12. However, in this case there is one participant for whom a large learning effect was associated with a large NU-NG rejection confidence difference, showing evidence for 
strategic use of conscious knowledge (point $0.63,0.41$ ). Interestingly, this was the person who reported the highest level of awareness in the post-test. Without this participant, the remaining group's data show a mild, non-significant negative trend, $r(7)=-0.38$, typical for the unaware group. It suggests that those people who have been classified as aware on the basis that they became aware of the regularity during post-test were not using this knowledge strategically during the test itself (which is consistent with their reports).
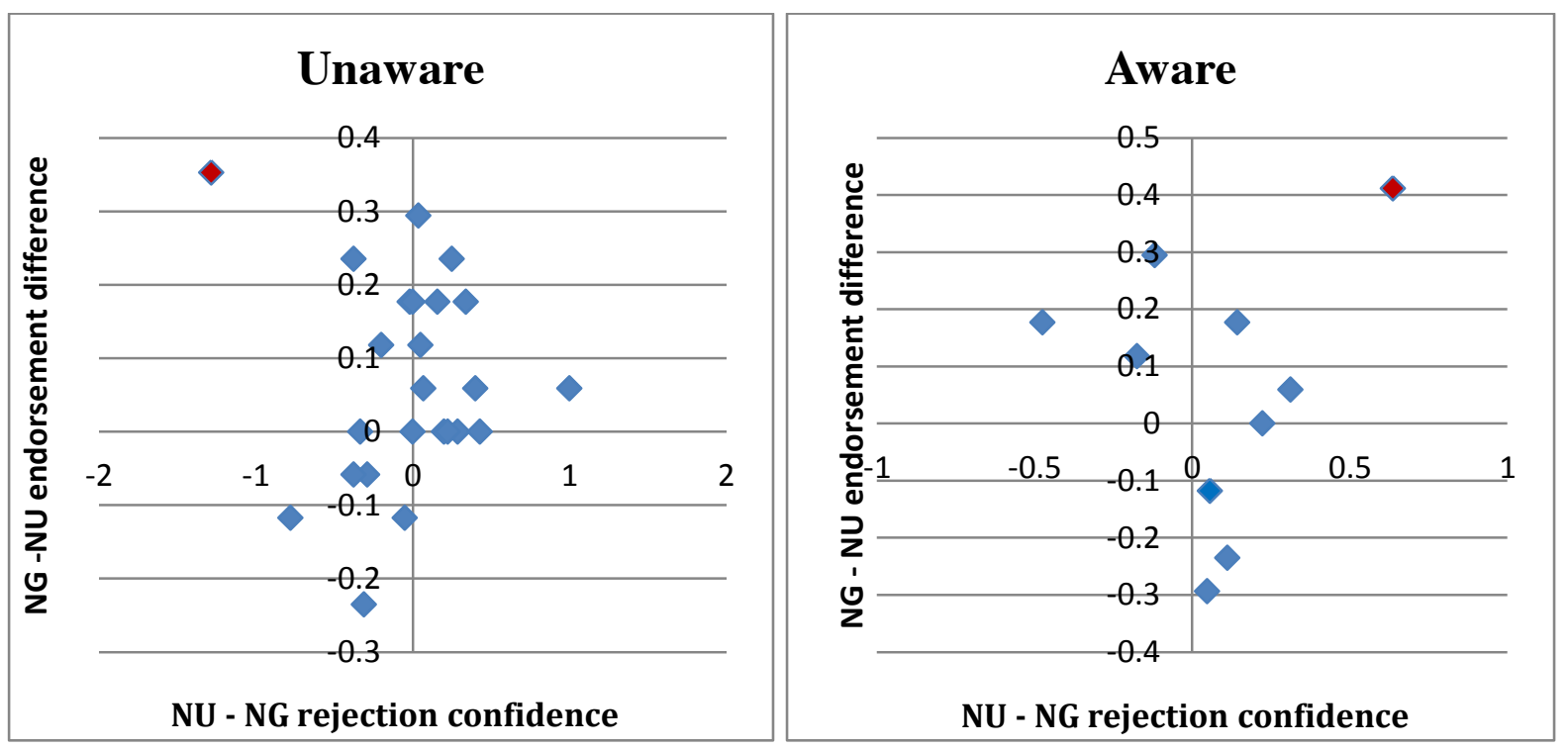

Figure 3.8. Confidence difference for NU-NG rejections and learning effect (NG-NU endorsement difference), for the a) unaware, b) aware group.

\subsubsection{Discussion}

Experiment $2 \mathrm{~b}$ replicated the central finding of Experiment $2 \mathrm{a}$, namely that people implicitly form semantic generalisations about the type of nouns which can follow novel verbs. The learning was revealed by a false-memory effect, wherein participants endorsed more new test items as familiar when they followed selectional preference patterns than when they did not. Awareness was assessed through an indirect measure involving participants' subjective source knowledge judgements. The analysis of source judgements (interpreted as a confidence scale) provided more convincing evidence than in Experiment $2 \mathrm{a}$ for nonconscious knowledge of the semantic regularity in the familiarity decision task.

The analysis of collocate types shows that introducing the article into the test phase items did have an impact on the results. Specifically, test items containing an abstract 
collocate were now endorsed at a higher rate than in Experiment $2 b$, and conversely test items with a concrete collocate were endorsed at a lower rate. This presumably reflects the effect of a match in the syntactic frame on familiarity decisions. That is, on seeing, for example, the NG item gouble the prestige, the fact that "the prestige" is a familiar fragment may have elevated the endorsement rate, and likewise for an ungrammatical item like mouten the prestige. Crucially, though, the effect of matching at this level did not alter the size of the grammaticality effect, although it was numerically smaller in this experiment (0.06 versus 0.11 in Experiment 2a).

Overall, regardless of whether the article did appear in the test (Experiment 2b) or did not appear (Experiment 2a), the fact that in the training phase the definite article almost invariably followed GOUBLE and POWTER could have suggested to the participants that these two words are more similar to each other than the other two words. Learning could have been aided by "syntactic bootstrapping" which contributed to the subsequent conceptual differentiation between novel words collocating with abstract and concrete nouns. Experiment 3 addresses this concern and the question of whether learning would still occur without syntactic cues. In this experiment, the training was modified in such a way that the definite article appeared in all contexts. Furthermore, in order to eliminate the possibility of syntactic bootstrapping, all concrete nouns used were mass nouns which did not contain the plural ' $s$ '.

\section{$\underline{\text { 3.4. Experiment } 3}$}

Participants: 33 people participated in the experiment, 32 were students at various UK universities, 1 was a university staff member.

\subsubsection{Materials}

Materials were similar to those in Experiment $2 \mathrm{a}$ and $2 \mathrm{~b}$. The word fonell was changed to conell to avoid the similarity with funnel, which could have suggested an action involving some tangible substance. Relatively long sentences were shortened. Crucially, any syntactic 
cues were removed. This involved removing the concrete plural nouns with an '-s' marker. All concrete nouns were mass nouns, such as sugar or magnesium. Furthermore, it was ensured that all target nouns, both abstract and concrete, were preceded by the definite article "the".

\subsubsection{Procedure}

The task was the same as in Experiment 2, but administered online. The post-test consequently required greater elaboration, due to the absence of the experimenter. Apart from the 8 multiple-choice sentences, the following questions were added, which appeared at the end of the post-test, one by one:

- Please describe what you have learnt about gouble, powter, conell and mouten.

- Did you notice any regular pattern concerning the novel words?

- If so, when did you realise this? In the first, second or final part of the experiment?

- Did you realise that in the first part the words gouble and powter always appeared with abstract nouns, such as authority, while conell and mouten with natural, tangible substances?

- If yes, when did you notice it?

- If you did notice that regularity, did you use this knowledge in the second part?

- Any other comments?

There were two versions of the test, with half the participants randomly allocated to each version. The nouns used to form the "New Grammatical" pairs in version 1 were included in the "New Ungrammatical" pairs in version 2, and vice versa.

\subsubsection{Results}

In the post-experiment questionnaire 11 out of 33 participants expressed awareness of the regularity and were classed as 'aware'. Two participants reported a wrong pattern and were excluded from the analysis due to potential confounds. The remaining 20 participants were classed as 'unaware'. 
Similarly to Experiment $2 \mathrm{a}$ and $2 \mathrm{~b}$, the analysis was performed with a correction for small numbers of responses, calculated as (number of correct responses +0.5$) /($ total number of responses +1 ). Table 3.6. shows the weighted mean endorsement rates for the two groups of participants.

Participants in both groups erroneously endorsed as familiar significantly more word pairs which followed the selectional preference pattern (New Grammatical, NG) than those that did not (New Ungrammatical, NU). For the aware group: $F(1,9)=10.70, p<0.05, \eta^{2}=$ 0.54; for the unaware group: $\mathrm{F}(1,18)=7.62, \mathrm{p}<0.05, \eta^{2}=0.30$. Also, in both groups, accuracy for the Old Grammatical (OG) items was significantly above chance; for the aware group, $\mathrm{t}(10)=2.93, \mathrm{p}<0.05$; for the unaware group, $\mathrm{t}(19)=3.36, \mathrm{p}<0.01$.

Table 3.6. Mean endorsement rates (standard error in parentheses).

\begin{tabular}{|l|l|l|l|}
\hline & NG & NU & OG \\
\hline Aware $(\mathrm{n}=11)$ & $.55(.038)$ & $.37 \quad(.045)$ & $.69 \quad(.065)$ \\
\hline Unaware $(\mathrm{n}=20)$ & $.50(.045)$ & $.44(.034)$ & $.63(.038)$ \\
\hline
\end{tabular}

$\mathrm{NG}=$ New Grammatical, NU = New Ungrammatical, $\mathrm{OG}=$ Old Grammatical

\section{Analysis of noun types}

Figure 3.9 depicts the difference between the endorsements of pairs with abstract and concrete nouns among the unaware group, calculated similarly as for Experiment $2 \mathrm{a}$ and $2 \mathrm{~b}$. 


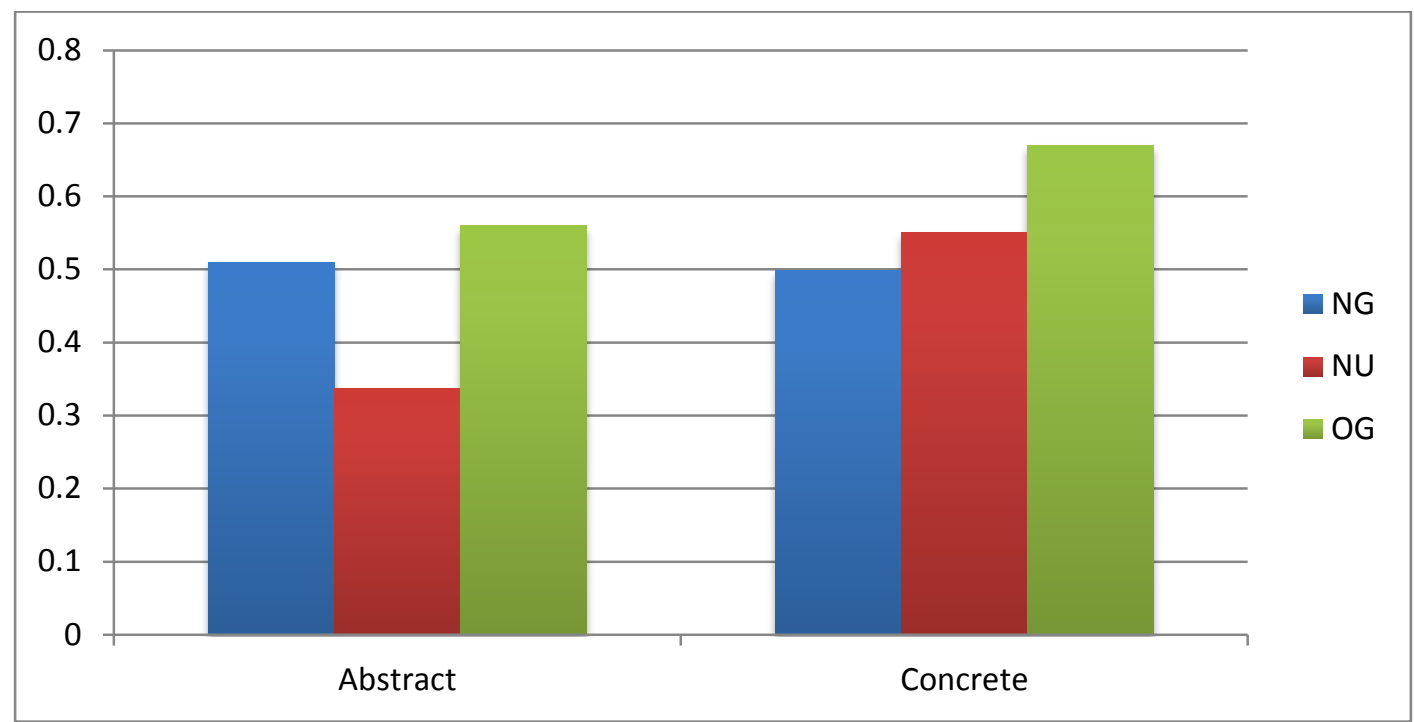

Figure 3.9. Mean endorsement rates for abstract and concrete collocates among the unaware participants.

An ANOVA was performed on the mean endorsement rates in the NG and NU conditions with grammaticality and collocate type as within-subject factors, and list as a betweensubjects factor. There was a main effect of grammaticality, $F(1,18)=7.62, p<0.05, \eta^{2}=030$, and a significant interaction between noun type and grammaticality, $\mathrm{F}(1,18)=10.95$, $\mathrm{p}<$ 0.01, $\eta^{2}=038$. It appears that in Experiment 3 participants differentiated NG and NU conditions only with pairs containing abstract nouns. Analysis of the recognition of the OG items also points to this conclusion $\mathrm{F}(1,18)=35.60, \mathrm{p}<0.001, \eta^{2}=0.67$.

\section{Analysis of source judgements}

The subsequent analysis will continue to focus on the unaware participants. Table 3.7 shows an even distribution of source knowledge attributions, similar to that obtained in the previous experiments. 
Table 3.7. Source proportions.

\begin{tabular}{|l|l|l|l|}
\hline \multicolumn{2}{|l|}{ Source proportions } & & \\
\hline & \multicolumn{1}{|c|}{$\mathrm{NG}$} & $\mathrm{NU}$ & $\mathrm{OG}$ \\
\hline guess & 0.302 & 0.316 & 0.208 \\
\hline intuition & 0.365 & 0.354 & 0.375 \\
\hline memory & 0.333 & 0.330 & 0.417 \\
\hline
\end{tabular}

Figure 3.10 shows the corrected endorsement rates by source. Similarly to Experiments 2a and $2 b$, the critical difference between NG and NU categories is mainly expressed in the source judgements attributed to memory, $\mathrm{F}(1,14)=8.93, \mathrm{p}<0.05, \eta^{2}=0.39$ (intuition, guess $\mathrm{F}<1.0)$

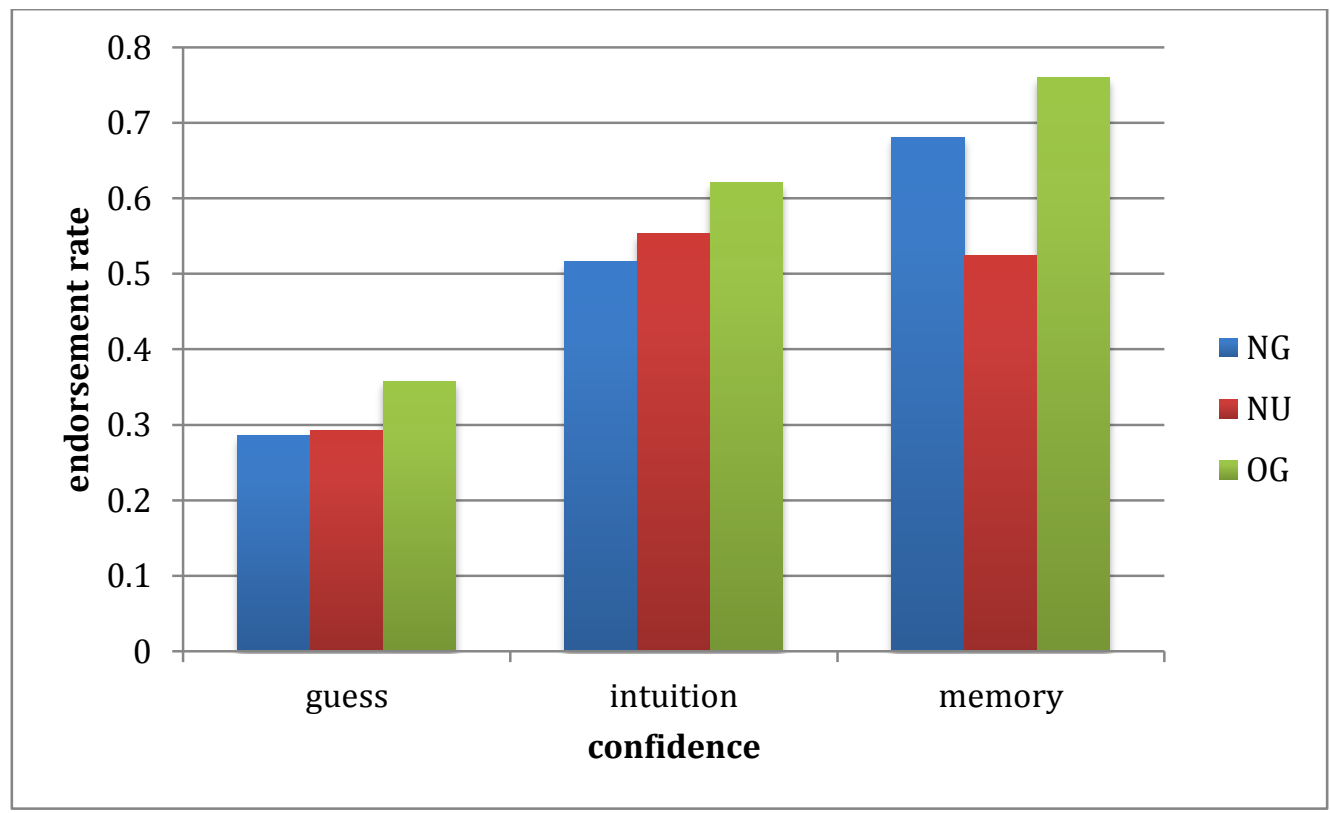

Figure 3.10. Endorsement rates by source, unaware participants.

Analogously to Experiments $2 \mathrm{a}$ and $2 \mathrm{~b}$, the issue of whether participants used any potential knowledge strategically was addressed by examining whether source judgements for correct rejections of the New Ungrammatical items denoted higher confidence than the judgements 
for rejections of the New Grammatical ones. For this reason, again, the guess-intuitionmemory scale was interpreted as a confidence scale with values of 1,2 and 3 . The results of this analysis are shown in Figure 3.11.
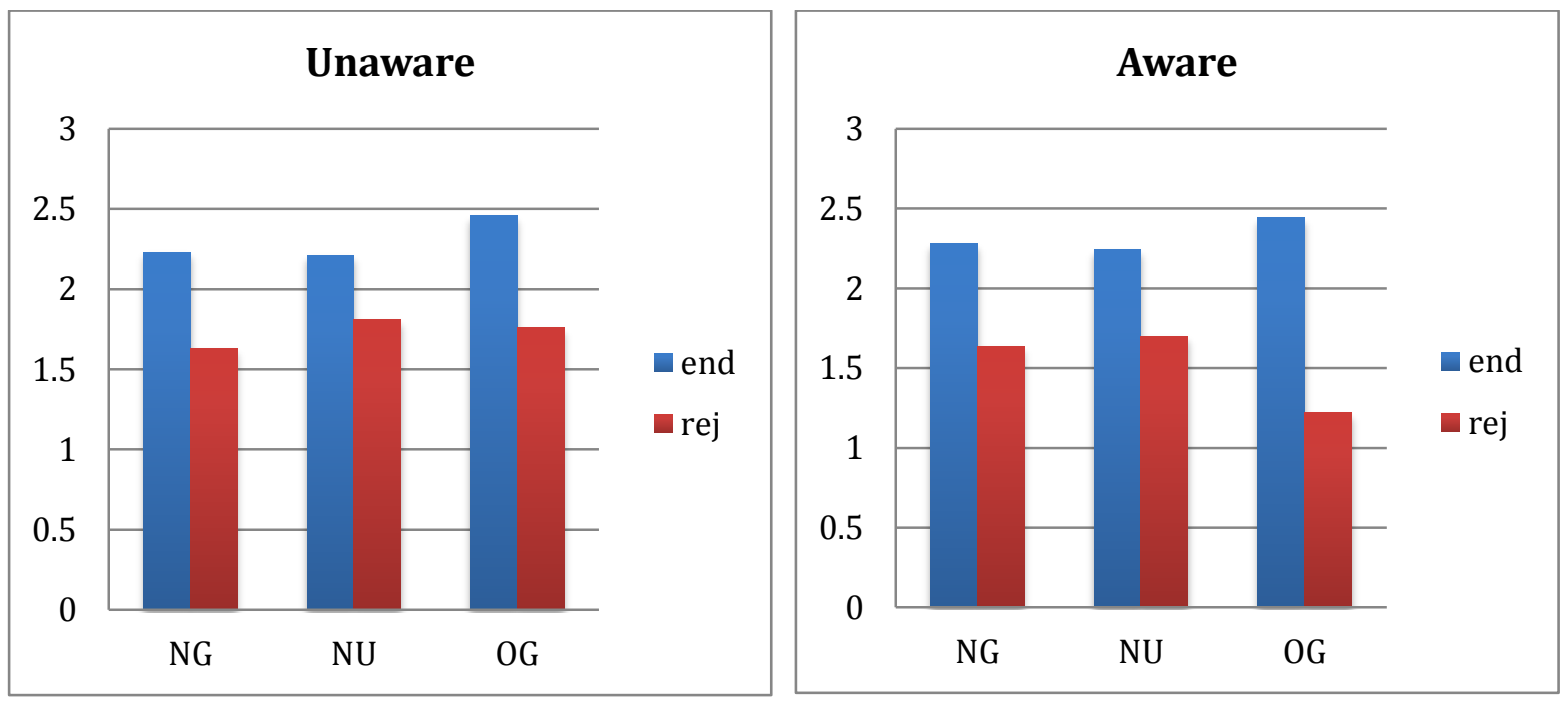

Figure 3.11. Mean confidence for endorsements and rejections.

An ANOVA was performed on the unaware participants' data on the mean confidence rates in the NG and NU conditions with grammaticality and endorsement/rejection as withinsubject factors, and list as a between-subjects factor. There was a main effect of endorsement/rejection, $\mathrm{F}(1,18)=9.56, \mathrm{p}<0.01, \eta^{2}=0.35$, and an interaction between endorsement/rejection and grammaticality, $F(1,18)=6.44, p<0.05, \eta^{2}=0.26$. The difference between confidence rejecting NU and NG, hypothesised to be an indicator of conscious knowledge, was significant: $\mathrm{F}(1,18)=11.94, \mathrm{p}<0.01, \eta^{2}=0.40$, indicating that this group of participants may have developed some conscious knowledge, which the final post-test did not pick up or which was still difficult to verbalise. Interestingly, this difference is not significant among the aware group. For each group, Old Grammatical (OG) pairs were endorsed with more confidence than pairs in the other conditions.

The higher confidence exhibited by participants in rejecting NU by comparison with NG suggests that participants developed some conscious knowledge, but may not mean that they used it strategically. That is, even though participants' confidence pattern suggests 
conscious knowledge, it does not mean that it correlated with the learning effect. Scatter plots of the NU-NG confidence difference and the learning effect are shown in Figure 3.12.
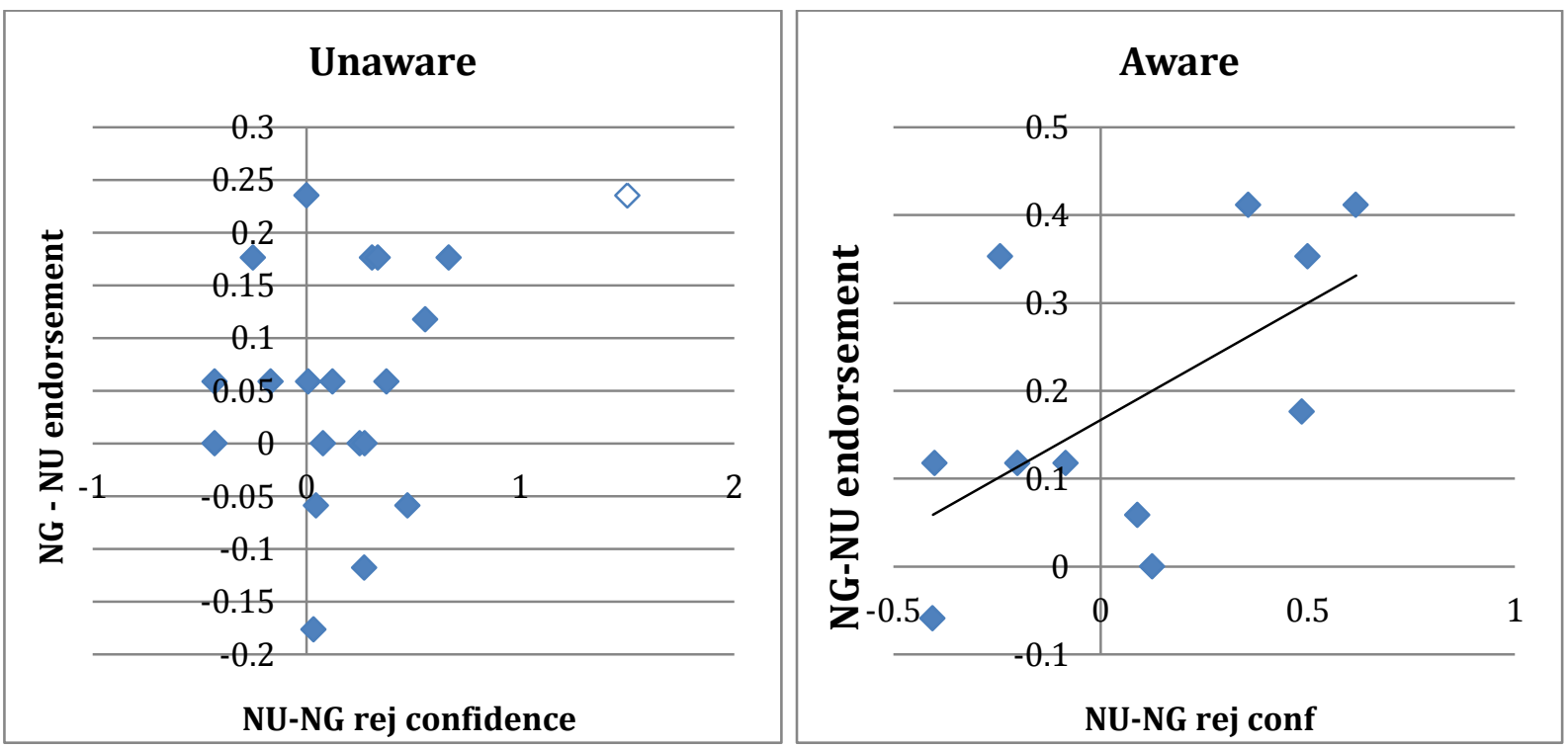

Figure 3.12. Confidence difference for NU-NG rejections and learning effect (NG-NU endorsement difference with Bayesian correction), for a) unaware, b) aware group.

Figure 3.12a for the unaware participants clearly shows that one participant was an outlier showing a particularly high difference between confidence for rejecting NU and NG. With this data point removed, the unaware participants showed no correlation between such confidence pattern and learning, $\mathrm{r}(17)=0.089$, n.s ${ }^{11}$. On the other hand, the correlation among the aware group approached significance, $r(9)=0.59, p=0.056$. This indicates a more consistent strategy in the performance of the aware participants than the unaware group. However, this must be interpreted with caution, because a test of the difference between two correlations (using the Fisher r-to-z transformation) showed no significant difference between two correlations, $\mathrm{z}=1.21, \mathrm{p}=0.23$ (but note the small sample size in the aware group). It is worth noting that aware participants could have become aware at different points of the experiment and might have used their knowledge consistently to a varying degree. Figure $3.12 \mathrm{~b}$ could be interpreted as showing two subgroups among the aware participants: those

\footnotetext{
${ }^{11}$ Prior to removal of this participant the correlation for the unaware group was $\mathrm{r}(18)=0.318, \mathrm{p}=0.17$
} 
whose confidence difference and learning effect go hand-in-hand (the four points in the upper right quadrant), and those who behave more like the unaware participants. This issue will be addressed again in the analysis of the explicit variant of this task (Experiment 5).

\subsubsection{Discussion}

This experiment provides some support for the hypothesis that people may be able to implicitly form semantic generalisations pertaining to novel verbs' selectional preference in the absence of syntactic cues. Although the learning effect was significant (NG-NU endorsement difference) among those participants who did not report awareness of any relevant knowledge in the post-test questionnaire, the confidence analysis revealed higher confidence for correctly rejecting NU than NG items. This may indicate some potentially conscious knowledge relevant to the tested regularity, which the participants did not verbalise in the post-test. However, this confidence difference did not correlate with the learning effect, therefore it could not be shown to augment participants' performance. The overall result remains inconclusive.

Methodologically, rather than reflecting implicit generalisations, participants' responses may have been influenced by the similarity between training and test items. In all of the experiments so far, the nouns used in test were highly similar in meaning to the ones in training, e.g. importance and significance, or authority and esteem. Although concrete nouns were also similar in training and test, for example oxygen and nitrogen, or calcium and potassium, they are not synonymous to a similar extent as the abstract nouns. The effect of synonymy could also account for the fact that participants mainly discriminated between correctly and incorrectly associated abstract nouns, whereas this effect was weaker among the concrete nouns. In other words, participants might simply have confused the words they saw with their synonyms, rather than learning the semantic regularity. Experiment 4 addresses the issue of whether what is observed here pertains to implicit learning or simply a similarity bias, by including a much more heterogeneous set of abstract and concrete nouns. 


\subsection{Experiment 4}

Participants: 70 participants, all native English speakers from the USA, were recruited online via the Amazon Mechanical Turk.

\subsubsection{Materials}

Materials were similar to those in Experiment 2a, 2b, and 3. The target nouns in training and test were changed so as to formulate more heterogenous sets of abstract and concrete nouns. The broadened category of abstract nouns included ones as different as: happiness, wisdom, impact, understanding. The category of concrete nouns was similarly broadened to include, for example: chocolate, luggage, metal and paper. The motivation for this change was that the test item should not be similar to an item that had collocated with the nonword in training (whereas in previous experiments it was). Just like in Experiment 3, all concrete nouns were mass nouns, and all target nouns were preceded by the definite article "the" in the training. The material is presened in Appendix D.

\subsubsection{Procedure}

The task followed the same procedure online as Experiment 3, except instead of the source judgements (guess, intuition, memory) participants indicated their confidence on a three-point scale (guess, somewhat confident, very confident). This removed the problem of translating the source judgements into a numerical scale. There were four lists (formed as in Experiment $2 \mathrm{a}$ and $2 \mathrm{~b}$ ) equally distributed among the participants.

\subsubsection{Results}

The data from two of the participants were excluded because they misunderstood the instructions. Of the remainder only 7 participants became aware of the regularity and the remaining 61 were classed as unaware. 
The mean endorsement rates, corrected with the Bayesian prior (as in the previous experiments) for the aware and unaware participants are shown in Table 5. Among the aware group the difference between the endorsement of the New Grammatical and New Ungrammatical items only approached significance, $F(1,6)=5.63, p=0.055, \eta^{2}=0.48$. Accuracy for the Old Grammatical (OG) items was significantly above chance, $\mathrm{t}(6)=4.04, \mathrm{p}$ $<0.01$.

Table 3.8. Overall endorsement rates (standard error in parentheses)

\begin{tabular}{|l|l|l|l|}
\hline & NG & NU & OG \\
\hline Aware $(\mathrm{n}=7)$ & $.63(.04)$ & $.42(.06)$ & $.71(.02)$ \\
\hline Unaware $(\mathrm{n}=61)$ & $.53(.02)$ & $.48(.02)$ & $.62(.02)$ \\
\hline
\end{tabular}

$\mathrm{NG}=$ New Grammatical, NU = New Ungrammatical, $\mathrm{OG}=$ Old Grammatical

Among the 61 unaware participants there was a significant difference between the NG and NU conditions, $F(1,57)=5.9, p<0.05, \eta^{2}=0.09$. Memory for the OG was highly significantly above chance, $\mathrm{t}(60)=5.3, \mathrm{p}<0.001$.

\section{Analysis of noun types}

Figure 3.13 depicts the difference between the endorsements (corrected with the prior) of pairs with abstract and concrete nouns among the unaware group. 


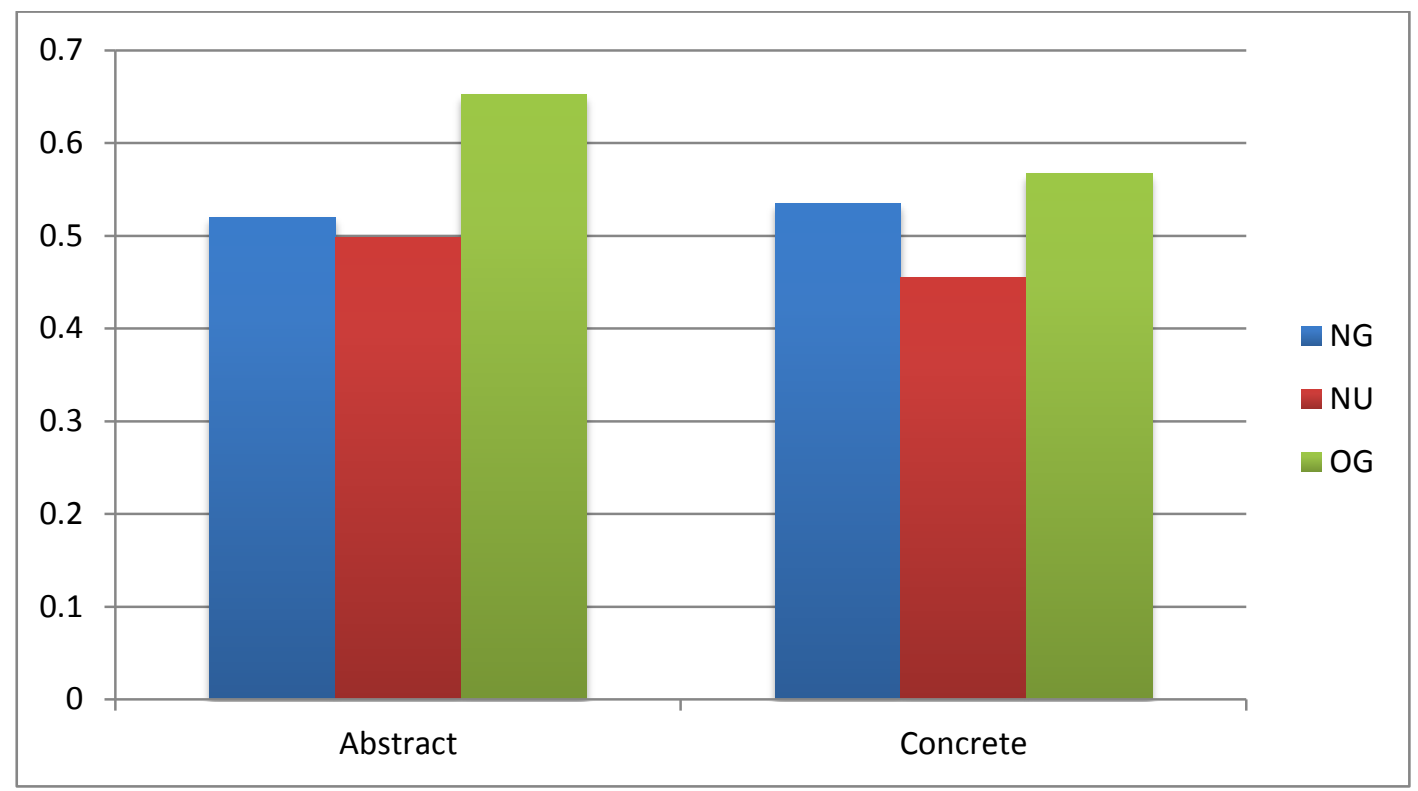

Figure 3.13. Endorsement rates for abstract and concrete collocates among the unaware participants.

An ANOVA was performed on the mean endorsement rates in the NG and NU conditions with grammaticality and collocate type as within-subjects factors and list as a betweensubjects factor. There was no main effect of collocate type, $\mathrm{F}<1.0$. There was no interaction between grammaticality and word type, $F(1,57)=1.61$. For the OG condition the items with abstract collocates were recognised significantly more often than those with concrete collocates, $\mathrm{F}(1,59)=5.98, \mathrm{p}<0.05, \eta^{2}=0.09$.

\section{Analysis of source judgements and confidence}

The subsequent analysis will mainly concern the unaware participants. Table 3.9 summarises the distribution of confidence judgements. Unlike in the previous experiments, the "moderately confident" category was used more often than the others. This can be explained by the fact that the stimuli are considerably more dissimilar from each other than in the previous experiments, resulting in participants' lower overall confidence. 
Table 3.9. Source proportions.

\begin{tabular}{|l|l|l|l|}
\hline \multicolumn{2}{|c|}{ Source proportions } & & \\
\hline & NG & NU & OG \\
\hline 1 & 0.237 & 0.234 & 0.189 \\
\hline 2 & 0.517 & 0.543 & 0.464 \\
\hline 3 & 0.246 & 0.223 & 0.347 \\
\hline
\end{tabular}

Endorsement rates broken down by confidence are presented in Figure 3.14. The rates were corrected with the Bayesian prior analogously to the previous experiments. Again, cases where there was no response in one category were excluded pairwise.

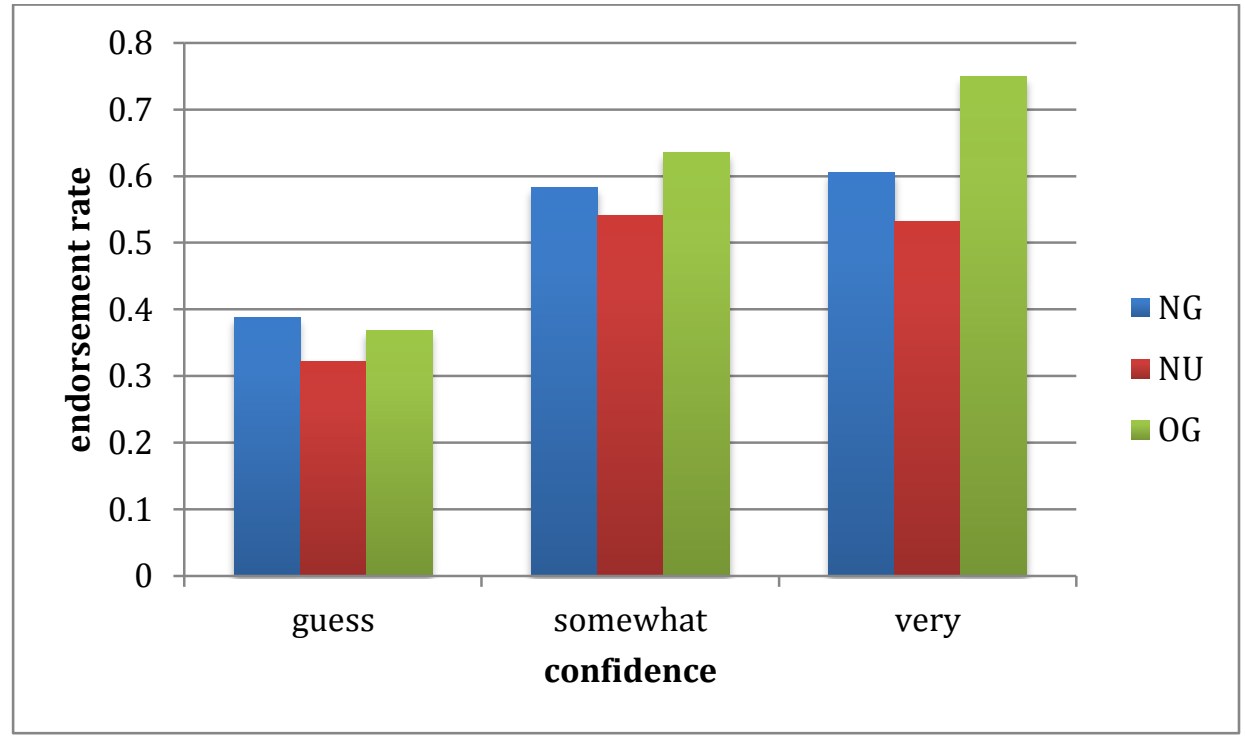

Figure 3.14. Endorsement rates by source, corrected, unaware participants.

An analysis of variance on the data from the NG and NU conditions showed a main effect of confidence, $\mathrm{F}(2,58)=11.2, \mathrm{p}<0.001, \eta^{2}=0.279$, but no interaction between confidence and grammaticality, $\mathrm{F}<1.0$. Tests of the grammaticality at each level of confidence were not significant: guess, $\mathrm{F}(1,38)=1.16$; somewhat confident, $\mathrm{F}(1,57)=1.67$; very confident, $\mathrm{F}<$ 
1.0, although note the reduced number of participants in these analyses because of missing data.

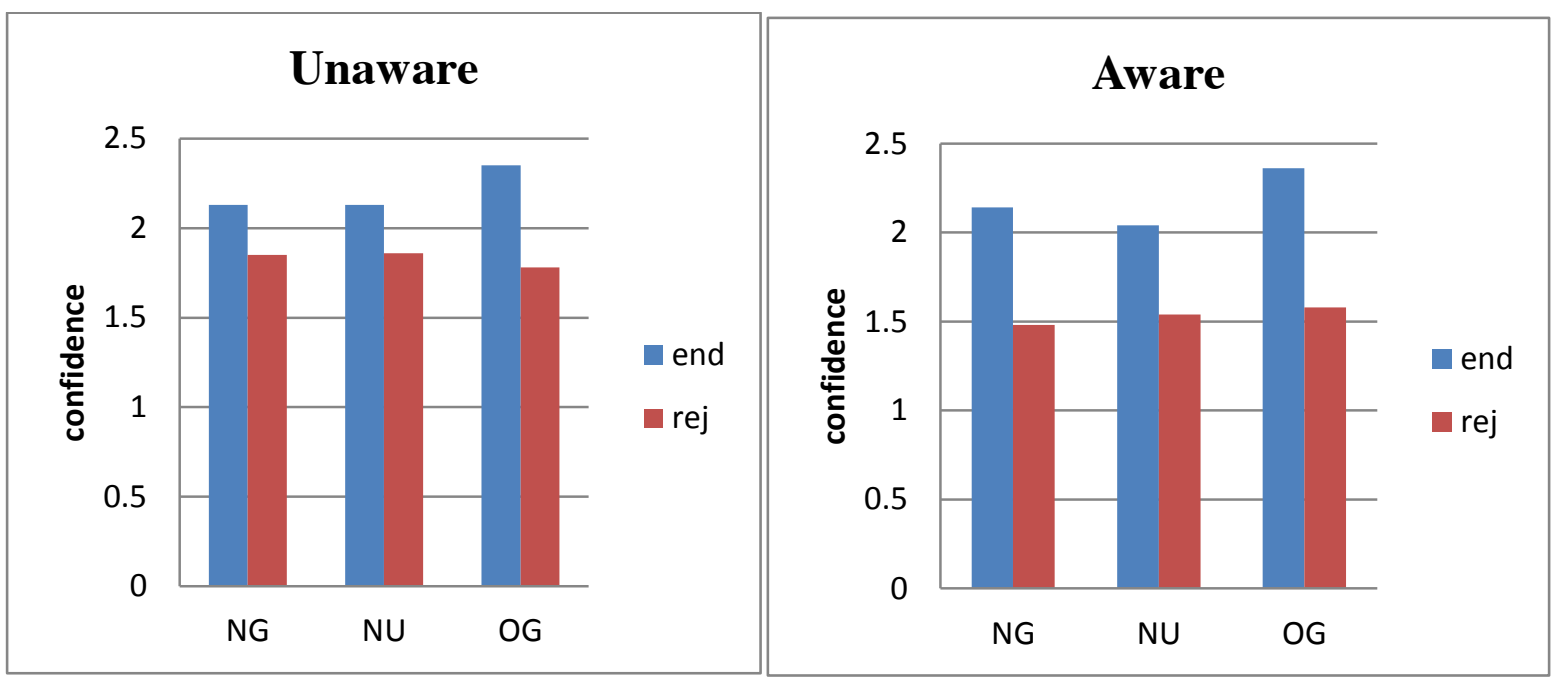

Figure 3.15. Mean confidence for endorsements and rejections.

An analysis of variance on the NG and NU data for the unaware participants showed only a main effect of endorsement/rejection, $\mathrm{F}(1,56)=25.4, \mathrm{p}<0.001, \eta^{2}=0.31$, and likewise for the aware group, $F(1,6)=2.34, \eta^{2}=0.82$. For both groups the confidence in rejections was almost identical in the NU and NG conditions, thus providing no evidence for the strategic use of explicit knowledge.

A correlation analysis of the learning effect (NG-NU endorsement difference) and confidence rejecting $\mathrm{NG}$ and $\mathrm{NU}$ further reinforced this conclusion for the unaware participants. A positive correlation was taken to indicate strategic use of some explicit knowledge. The scatter plots of these correlations are presented in Figure 3.16. The unaware group turned out to show a negative trend, $\mathrm{r}(59)=-0.33, \mathrm{p}<0.01$. In accordance with our prediction, the aware group showed the predicted positive trend, $r(5)=0.79, p<0.05$, indicating strategic application of knowledge. However, note that the correlation appears to be due to just two participants with a large NG-NU confidence difference and a large learning effect. Overall, the confidence analysis in this experiment produced similar results to the analyses in Experiments 2 and 3 where "confidence" was derived indirectly from source judgments. 

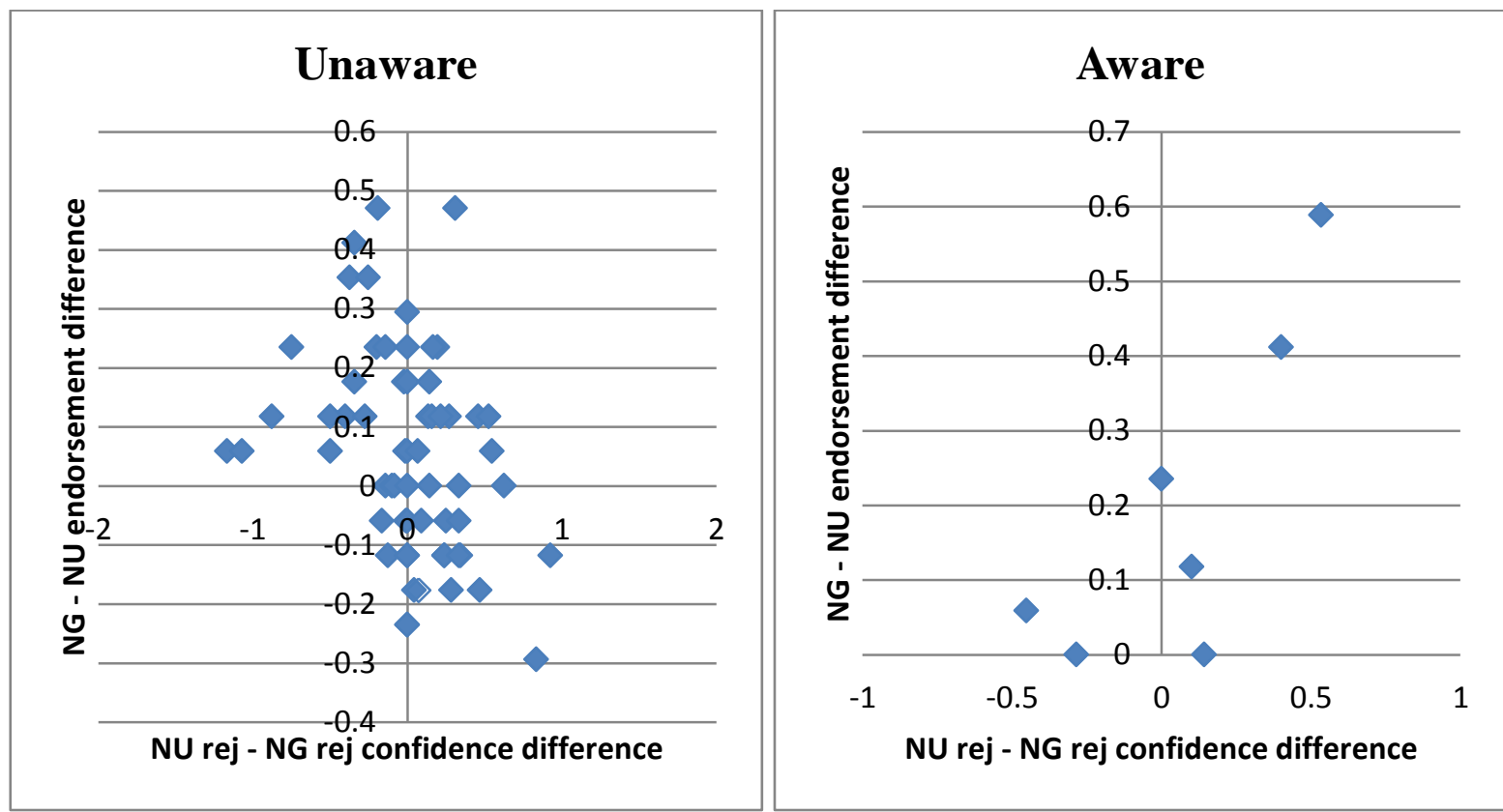

Figure 3.16 Confidence difference for NU-NG rejections and learning effect (NG-NU endorsement difference), for the unaware and aware groups.

\subsubsection{Discussion}

Overall, the pattern of responses in Experiment 4 has provided further support for the notion that people are able implicitly to form semantic generalisations pertaining to the selectional preferences of novel verbs. They are shown to do this even when the material includes semantic categories with highly heterogeneous words, such as for abstract words: happiness, wisdom, impact, understanding, and for concrete words: chocolate, luggage, metal, paper. However, although the numerical size of the effect in the unaware group was comparable to that in experiment 3 , the effect size was much smaller $\left(\eta^{2}=0.30\right.$ in experiment 3 versus $\eta^{2}=$ 0.09 in experiment 4), and achieved the same level of significance despite being based on a much larger sample size (20 unaware in experiment 3 and 61 unaware in experiment 4). There is some indication, therefore, that when the similarity between the test items and the training items was reduced, the size of the learning effect was reduced also. Thus, although the semantic generalisation that is learned is broad enough to survive the reduction in similarity, this does not mean that semantic similarity is completely irrelevant to the effect, and it cannot be claimed that participants are necessarily learning an abstract 'concrete' 
versus 'abstract' distinction as such. Interestingly, the decrease in effect size across experiments 3 and 4 is only marked for the unaware groups. The aware groups showed comparable effect sizes $\left(\eta^{2}=0.54\right.$ in Experiment 3 and $\eta^{2}=0.48$ in Experiment 4). This might suggest that in both experiments the aware participants were just as likely to conceive of the underlying regularity in terms of an abstract 'concrete vs. abstract' distinction that was immune to the reduction in semantic similarity between items. This issue will be discussed further in the General Discussion to this chapter.

The conclusion that the learning which has occurred is implicit crucially hinges on the methodology used. Here, the task is based on the false memory phenomenon. It is assumed that the creation of false memories is an automatic process happening involuntarily. To assure the accuracy of this prediction, and therefore the methodology applied here, Experiment 5 was devised. Another group of participants was tested on an explicit variant of the task used in Experiment 4. In the new variant, the target regularity was revealed upfront in the initial instructions. The test was still unexpected, just like in the previous experiments. We tested whether participants naturally used their explicit knowledge strategically in the test. If so, endorsements of New Ungrammatical items would be close to zero. If not, the pattern of results would resemble that in the previous experiments. This last group was also predicted to outperform the implicit groups on the final, short post-experiment test.

\subsection{Experiment 5}

Participants 24 native speakers of English were recruited online from the USA via the Amazon Mechanical Turk. The mean age was 34.6 years. 9 of them were male.

\subsubsection{Materials}

The materials were exactly the same as in Experiment 4 (see: Appendix D), only the instructions revealed the target regularity upfront. Participants saw the following text: 
'You will see sentences on the screen. Please read them carefully.

They are taken from various newspaper articles, each sentence from a different article.

Some of them contain novel words in CAPITALS. These words are MOUTEN, CONELL, POWTER and GOUBLE. They are acting as verbs.

MOUTEN means to increase an abstract thing (e.g. MOUTEN the approval), POWTER means to increase a concrete thing (e.g. POWTER the aluminium)

CONELL means to decrease an abstract thing (e.g. CONELL the speed), GOUBLE means to decrease a concrete thing (e.g. GOUBLE the rubber)

As you read each sentence notice how the novel words are used and this will reinforce their meanings in your mind. You will be tested on their meanings at the end.

In addition, for each sentence:

1) Please indicate whether the word in CAPITALS indicates roughly 'becoming less of' (- ) or 'becoming more of' (+).

2) Imagine what each article is about and indicate whether it talks about issues which are personally Important or Unimportant to you.

Bear in mind that your main task is to learn the meanings of the novel verbs (whether they mean increase or decrease and whether they go with abstract or concrete things).'

\subsubsection{Procedure}

The experiment followed exactly the same procedure as Experiment 3 and Experiment 4. There were four versions equally distributed among participants.

\subsubsection{Results}

The mean endorsement rates and significant deviations from chance are presented in Table 3.10 . 
Table 3.10. Overall endorsement rates (standard errors in parentheses)

\begin{tabular}{|l|l|l|l|}
\hline & NG & NU & OG \\
\hline All $(\mathrm{n}=24)$ & $.57(.048)$ & $.38(.052)$ & $.64(.042)$ \\
\hline Perfect post test $(\mathrm{n}=15)$ & $.62(.059)$ & $.32(.077)$ & $.68(.051)$ \\
\hline Not perfect post test $(\mathrm{n}=9)$ & $.52(.082)$ & $.43(.076)$ & $.59(.076)$ \\
\hline
\end{tabular}

$\mathrm{NG}=$ New Grammatical, NU = New Ungrammatical, $\mathrm{OG}=$ Old Grammatical

Participants endorsed significantly more New Grammatical pairings than the New Ungrammatical ones, $F(1,20)=9.31, p<0.01, \eta^{2}=0.32$. Accuracy for the Old Grammatical $(\mathrm{OG})$ items was significantly above chance, $\mathrm{t}(23)=3.73, \mathrm{p}<0.01$. This was the case both for the participants who were perfectly accurate in the final post-experiment test of explicit knowledge, $\mathrm{F}(1,13)=8.59, \mathrm{p}<0.01, \eta^{2}=0.4$, and for those who were not perfectly accurate at the end, $\mathrm{F}(1,7)=5.76, \mathrm{p}<0.05, \eta^{2}=0.45$. The learning effect was numerically larger in the perfect group, but the interaction between group and grammaticality was not significant, $\mathrm{F}(1,20)=2.95, \mathrm{p}>0.1$.

\section{Analysis of word types}

Figure 3.18 depicts the difference between the endorsements of pairs with abstract and concrete nouns. 


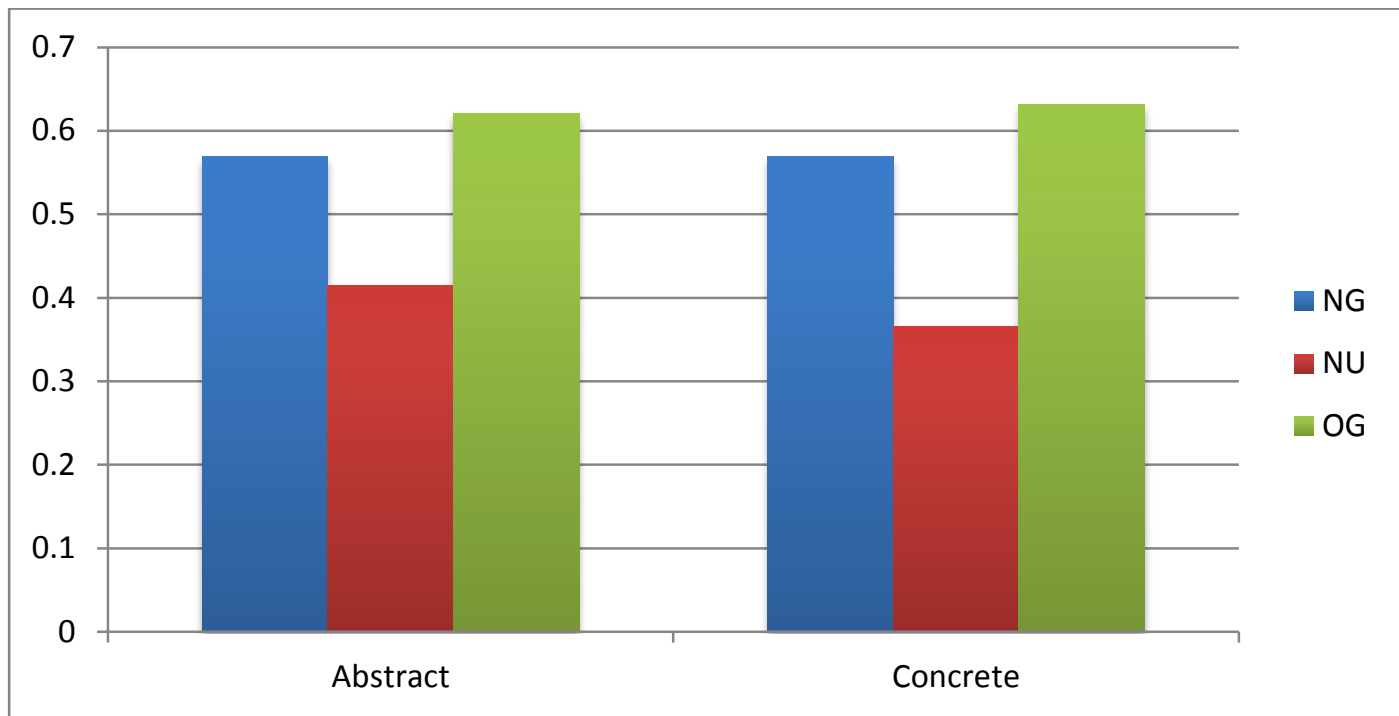

Figure 3.18. Endorsement rates for abstract and concrete collocates.

An ANOVA was performed on the mean endorsement rates in the NG and NU conditions with grammaticality and collocate type as within-subjects factors. The main effect of collocate type was not significant, $\mathrm{F}<1.0$, nor was the interaction between collocate type and grammaticality, $\mathrm{F}<1.0$. The $\mathrm{OG}$ condition also showed no effect of collocate type, $\mathrm{F}<1.0$.

\section{Analysis of source judgements ${ }^{12}$}

Table 8 shows proportions of participants' confidence judgements. As expected, source proportions reveal very few responses attributed to the lowest confidence.

Table 3.11. Proportions of confidence judgements.

\begin{tabular}{|l|l|l|l|}
\hline \multicolumn{2}{|l|}{ Confidence proportions } & & \\
\hline & NG & NU & OG \\
\hline guess & 0.211 & 0.164 & 0.161 \\
\hline somewhat & 0.435 & 0.391 & 0.323 \\
\hline very & 0.354 & 0.445 & 0.516 \\
\hline
\end{tabular}

\footnotetext{
${ }^{12}$ Equivalent to source analysis in Experiments 2a, $2 \mathrm{~b}$ and 3.
} 
Figure 3.19 shows the endorsement rates by confidence. Since some participants may have small numbers of responses attributed to different confidence judgements, just like in Experiments 2a, 2b, 3 and 4, the same Bayesian correction was applied. The critical difference between the NG and NU conditions is revealed only in the 'very confident' category, $\mathrm{F}(1,15)=8.18, \mathrm{p}<0.05, \eta^{2}=0.353$. This pattern follows the trend from the previous experiments, although differs from its twin in Experiment 3. This difference is not surprising taking into account the fact that explicit knowledge of the semantic rule could naturally raise the overall confidence of responses.

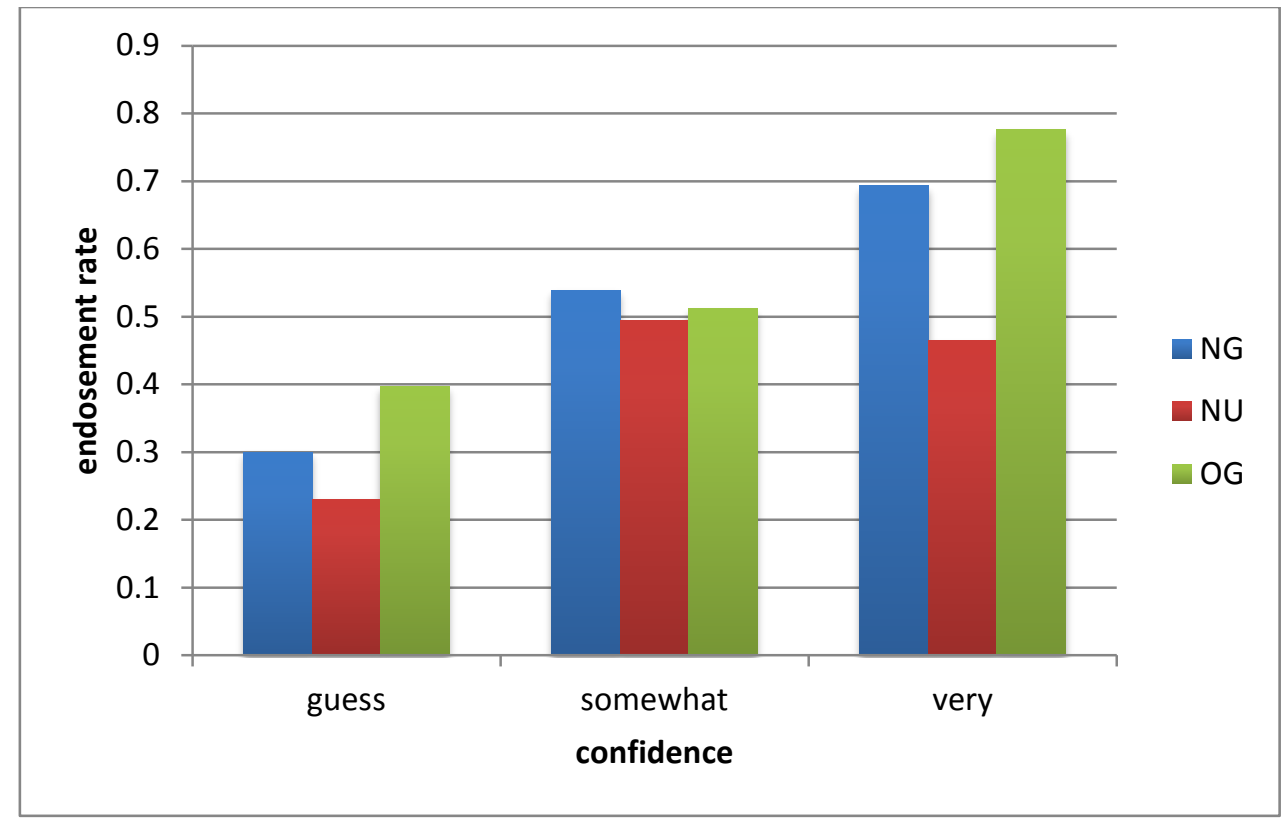

Figure.3.19. Endorsement rates by confidence source.

Similarly to the previous experiments, an analysis of confidence was performed to see whether participants used their knowledge strategically. Again, this is hypothesised to result in higher confidence for correctly rejecting New Ungrammatical items than rejecting New Grammatical items. Figure 3.20 shows mean confidence for endorsements and rejections. 


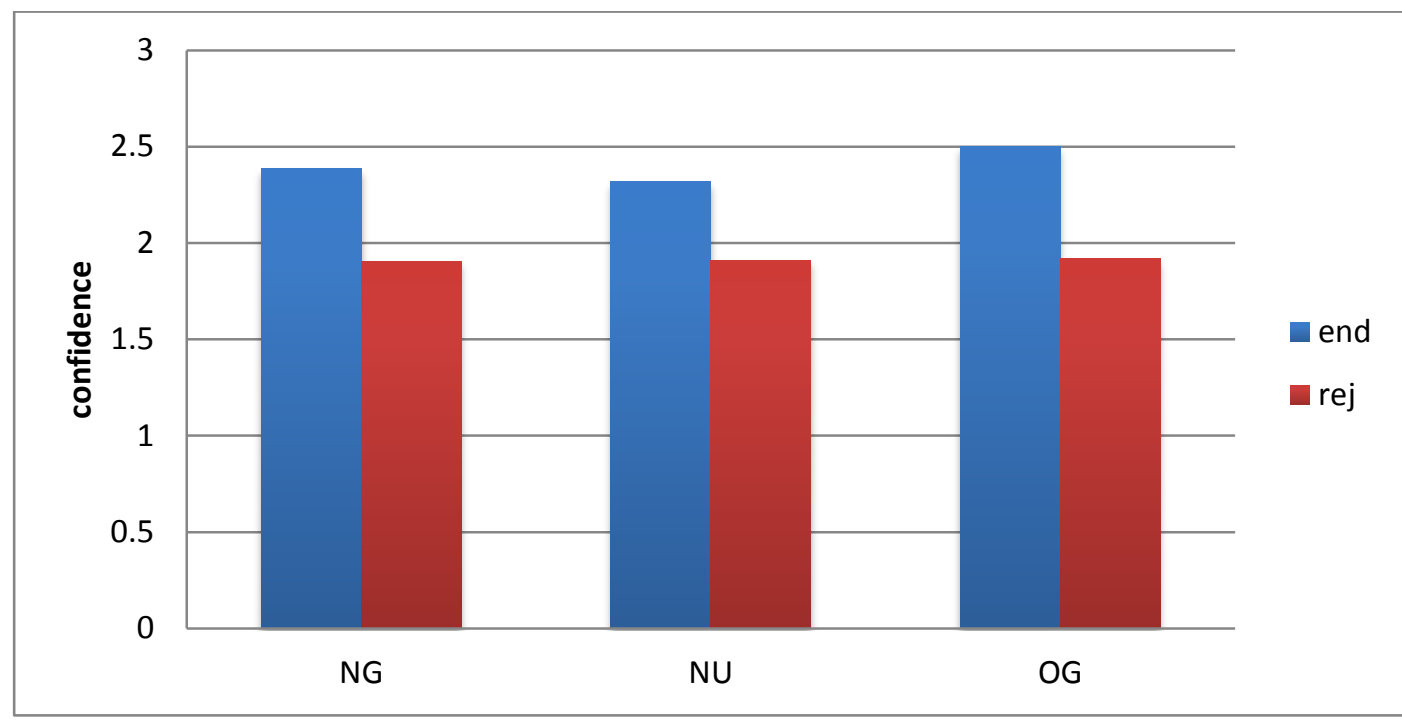

Figure 3.20. Mean confidence for endorsements and rejections.

An ANOVA was performed on the confidence data with grammaticality and endorsement/rejection as within-subjects factors, and list as a between-subjects factor. There was a main effect of endorsement/rejection: $F(1,15)=3.65, p<0.01, \eta^{2}=0.488$, but no interaction between endorsement/rejection and grammaticality, $\mathrm{F}<1$. This pattern was similar among both groups, those who were perfect and those who were not perfect on the post-test. Crucially, the difference in confidence for rejecting New Ungrammatical items and rejecting New Grammatical items was not significant in either group (perfect: $F(1,12)=1.09$, not perfect $F<1.0) .{ }^{13}$ This is a crucial finding, indicating that even the explicit group did not apply their knowledge strategically on the current false-memory task. Arguably this validates the task as a measure of semantic implicit learning effects.

As expected, the correlation analysis between the learning effect (NG-NU endorsement difference) and confidence pattern predicted to reflect conscious responses (NU rejection - NG rejection confidence) among all participants in Experiment 5 revealed a significant pattern, $\mathrm{r}(22)=0.497, \mathrm{p}<0.05$.

\footnotetext{
${ }^{13}$ Due to reduced numbers of participants only the rotation of the collocates was used as a list factor here.
} 


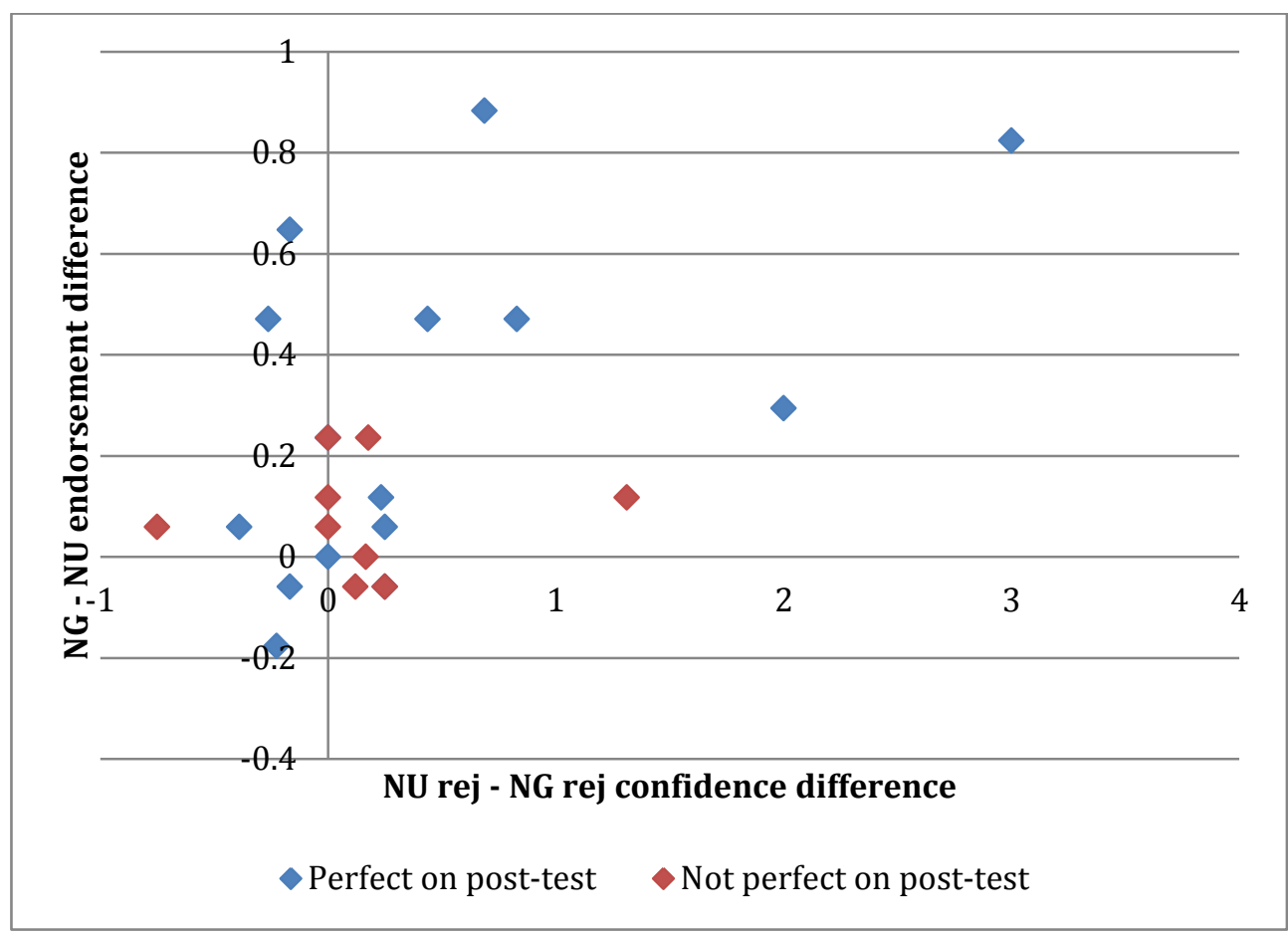

Figure 3.21. Confidence difference for NU-NG rejections and learning effect (NG-NU endorsement difference).

However, an interesting difference is revealed when contrasting the group who were perfect on the final test with those who were not (see Figure 3.21). The correlation between the learning effect and confidence rejecting NG-NU was significant for those participants who were perfect on the post-test, $\mathrm{r}(13)=0.523, \mathrm{p}<0.05$, and negligible for those who were not, $\mathrm{r}(7)=0.064$. Those who were not perfect on the post-test reveal behaviour similar to the implicit groups in previous experiments. This suggests that the correlation analysis used here can be usefully applied as an indirect measure of strategic use of conscious knowledge during the test. However, this must be interpreted with caution, because a test of the difference between two correlations (using the Fisher r-to-z transformation) showed no significant difference between two correlations, $\mathrm{z}=1.21, \mathrm{p}=0.23$, possibly due to the small sample sizes. This is reminiscent of the finding in Experiment 3 (p. 83).

Interestingly, even amongst participants who were perfect on the post-test, the correlation is driven by people in the upper right quadrant. Still, there are people who do not show a learning effect (even though they know the system, they fail to apply it in the familiarity task), and there are two who show large learning effects but no confidence difference - which can be taken to indicate that they are using implicit knowledge (i.e. they 
have the explicit knowledge but they are not using it intentionally in the test - there are a few cases like these in the previous 'aware' group scatter plots as well). To conclude, even when people have explicit knowledge of the rule they do not necessarily use that knowledge strategically in the recognition memory task.

\subsubsection{Discussion of the English experiments 2-5}

Overall, the above experiments suggest that people form semantic generalisations about nouns which can follow novel verbs. A false-memory task was adapted to measure learning as difference between erroneous endorsement of new items which followed the correct regularity and the new items which did not. The method combined a false-memory procedure with subjective measures of awareness and confidence. The overall analysis of mean confidence in different categories revealed no differences between those who were able to report the system at the end (aware), and those who were not. This, combined with the results from the explicit group (Experiment 5), indicates that in a false-memory procedure people in general do not use their knowledge strategically, even if such knowledge is provided explicitly. The procedure therefore offers a useful and valid method to address implicit learning, as it minimises the often quoted methodological counter-arguments to implicit learning research, including the fact that people may construct knowledge which they don't report, or have some partial relevant knowledge which is responsible for the effect. The measure which turned out to be very sensitive to whether a group used knowledge strategically or not was a correlation analysis between the learning effect and the confidence pattern hypothesised to reflect conscious knowledge (higher for rejecting NU than rejecting $\mathrm{NG})$. In experiments $2 \mathrm{a}, 2 \mathrm{~b}$ and 4 , and 5 there was a significant correlation or trend among the aware groups, but not the unaware groups.

Although collectively the experiments seem to point to the conclusion that implicit semantic generalisations are formed through normal sentence reading, and the proposed methods for detecting conscious knowledge suggest that the process is indeed implicit, a counter-argument of a different kind can be put forward. We might in fact not be dealing with learning as such, but with identification of translation equivalents for the target words - even at a subconscious level. To test whether the effect was facilitated by translation/recognition of 
equivalents, the following experiments attempt to replicate the above findings using Polish translations of the materials. Although Polish does have equivalent words for 'diminish', 'deplete', 'increase' and 'add', these words would not be followed naturally by the same nouns as in English. Indeed, in the following experiments people often reported that the sentences sounded somewhat unnatural.

\section{$\underline{\text { 3.7. Experiment 6a }}$}

Participants: 24 Polish students of the Jagiellonian University in Krakow and Krakow University of Economics. 15 of them were male.

\subsubsection{Materials}

To allow for comparisons with the previous experiments, the materials comprised the translations of the training and test stimuli from Experiment 2a. Unlike in the English experiments, Polish speakers could not substitute each novel word for a single existing Polish word. In fact, the sentences were checked by eight Polish native speakers in order to ensure that no one substitute for each novel word could be identified. The four novel verbs were: uzopyć, staleć, wpełać, and zomić. Care was taken to ensure that they did not resemble any existing Polish words, which could suggest a relation to abstract or concrete objects. As in Experiments 3, 4 and 5 the absence of syntactic cues was ensured and all target nouns appeared in the same Dative case. Keeping the case the same resulted in some sentences sounding 'slightly unnatural', though not ungrammatical. This fact should have also made it more difficult for participants to look for single translation equivalents of the novel words.

An example training item:

Fakt, że telewizja jest nadal najpolularniejszym z mediów nie oznacza, że Fact that the TV is still the most popular of the media does not mean that

moża GOSZYĆ znaczenie radia w kreowaniu opinii publicznej one can GOSZYĆ the meaning[+Dat] of the radio in shaping the public opinion. 


\subsubsection{Procedure}

The task followed the same procedure as the Experiments 2a and 2b. Participants were tested in person and performed the task on a computer, with the stimuli displayed in Superlab 4.0.

\subsubsection{Results}

8 out of 24 participants expressed at least some awareness of the regularity, or made potentially helpful observations. They were classified as the 'aware' group. The remaining 16 participants were classified as the 'unaware'. As in the previous experiments, the analysis was performed on means corrected for small number of responses (unless otherwise stated). The mean endorsement rates for the aware and unaware participants are shown in Table 3.12 below.

Table 3.12. Mean endorsement rates (standard error in parentheses)

\begin{tabular}{|l|l|l|l|}
\hline & NG & NU & OG \\
\hline Aware $(\mathrm{n}=8)$ & $.44(.039)$ & $.41 \quad(.077)$ & $.53 \quad(.058)$ \\
\hline Unaware $(\mathrm{n}=16)$ & $.55(.035)$ & $.49(.047)$ & $.71 \quad(.030)$ \\
\hline
\end{tabular}

NG = New Grammatical, NU = New Ungrammatical, $\mathrm{OG}=$ Old Grammatical

Unlike in the previous experiments, here neither group exhibited a significant difference between their endorsement of the NG and NU type items: aware $F(1,4)<1.0$, unaware $F(1$, $12)=1.63, p=0.23, \eta^{2}=0.12$. However, note that the numerical effect size is the same as in Experiment 3 (p.79), suggesting the possibility there may be an effect of grammaticality on a larger sample size. Accuracy for the correct endorsement of the Old Grammatical (OG) items was significantly above chance only among the unaware group, single sample t-test: $\mathrm{t}(15)=$ $6.90, \mathrm{p}<0.001$. 


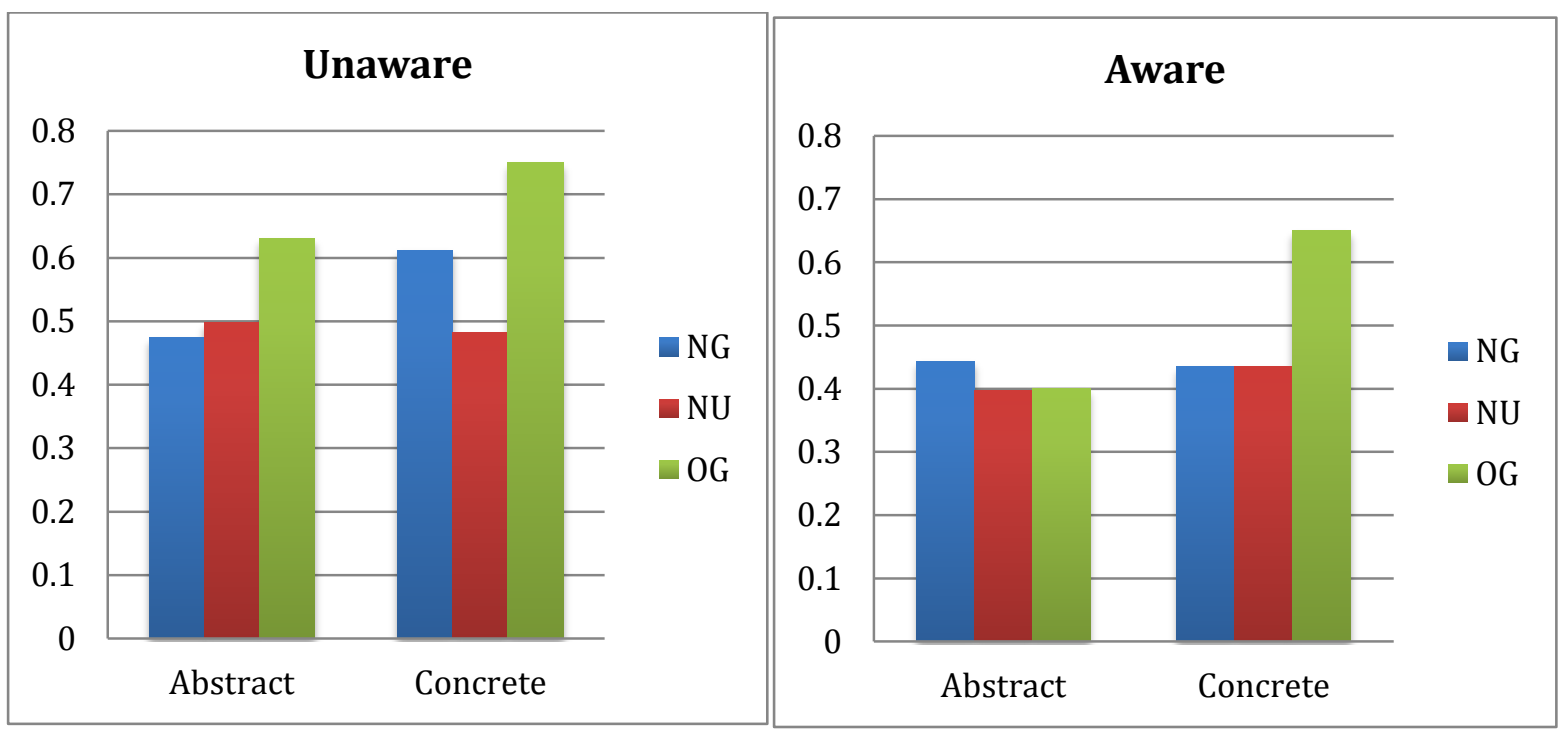

Figure 3.22. Endorsement rates for abstract and concrete collocates.

An ANOVA was performed on the mean endorsement rates in the NG and NU conditions with grammaticality and collocate type as within-subjects factors. The main effect of collocate type was not significant, nor was the interaction between collocate type and grammaticality.

\subsubsection{Discussion}

Unlike the previous experiments, here Polish native speakers did not reveal a statistically significant evidence for implicit learning of the selectional preferences of four novel verbs (though notice the raw effect size). It was particularly surprising that the overall memory for OG should be relatively low among the aware people, who expressed some awareness of the selectional preference rule. The 'by noun type' analysis revealed that this was the case mainly due to low endorsement rates for the combinations with abstract nouns. These particular combinations turned out to be especially difficult to remember, despite participants having some relevant rule knowledge. 
A potential reason why this situation occurred may concern the forms of the novel words. They were indeed devised to be as dissimilar as possible to any feasibly fitting, existing Polish words. In the light of Monaghan, Christiansen, \& Fitneva, (2011), this level of artificiality may have hindered participants' differentiation between the noun types following the novel verbs. It has been shown that categorising is impeded by arbitrariness of the word form. Categorisation requires some non-arbitrary cues, which may be in the form of, e.g. onomatopoeia (Langdon, 1984, mentioned in Monaghan et al. 2011), phonaesthemes (e.g. words referring to large sizes tend to contain low vowels: large/vast/humongous and those referring to small sizes contain high vowels: little, tiny; Monaghan et al. 2011), or synaesthetic relations between certain sounds and meanings. Nygaard, Cook, \& Namy, (2009) showed facilitation of sound-meaning relationships on the learning of Japanese words by English monolinguals. Participants showed learning after being exposed to matching equivalents (e.g. akurai and bright), and, to a lesser extent, opposites (e.g. akurai and dark), but not random pairings. These findings suggest that learners do make use of the nonarbitrary links between sound structure and word meaning.

For current purposes it would not be desirable if the novel words intrinsically suggested abstract/concrete context (e.g. word forms resembling pour, chop may prime concrete nouns or escalate, reduce abstract ones). But the problem of arbitrary novel word forms may still be alleviated by creating them in such a way as to plausibly imply an increasing/decreasing meaning, which is a separate dimension of meaning to the one being tested (abstract/concrete object). The task instructions required participants to focus on the increase/decrease distinction, hence the novel words should plausibly indicate it. A norming study was first conducted to establish a set of words with these characteristics.

\subsection{Norming study 1}

Participants: 12 Polish native speakers, average age 28. 


\subsubsection{Materials}

The materials comprised of a list of 36 nonsense but phonotactically possible Polish words, including the 4 words used in Experiment 6a.

\subsubsection{Procedure}

Participants were asked to rate each word on a scale of -5 to 5 in terms of whether it "sounds" like decreasing/removing or rather increasing/adding. -5 indicated 'definitely decreasing/removing', 0 indicated the word sounded neutral, and +5 indicated definitely increasing/adding. The task was performed online using SurveyMonkey ${ }^{\circ}$.

\subsubsection{Results}

Participants' ratings are shown in Table 3.13. The average word ratings spanned between -3.33 and +3.75 . Out of the four words used in Experiment $6 \mathrm{a}$, only one turned out to be moderately suggestive of increasing (wpełać: +1.83 ), whereas the other words were clearly neutral in that respect (uzopyć -0.92, staleć -0.5, zomić -0.08).

\subsubsection{Conclusion}

As became clear, the words used in Experiment 6a were very neutral in terms of potential increase/decrease meaning. It should be of interest to replicate Experiment $6 \mathrm{a}$ using the two most 'increase-like' and two most 'decrease-like' words from the list. This will test whether the failure to induce learning could have been caused by arbitrariness of the novel word forms. 
Table 3.13. Polish non-words norming study results.

\begin{tabular}{|c|c|c|c|c|c|c|c|c|c|c|c|c|c|}
\hline & -5 & -4 & -3 & -2 & -1 & 0 & 1 & 2 & 3 & 7 & 5 & & \\
\hline pokrawać & $25.0 \%(3)$ & $25.0 \%$ (3) & $25.0 \%(3)$ & $8.3 \%(1)$ & $16.7 \%(2)$ & $0.0 \%(0)$ & $0.0 \%(0)$ & $0.0 \%(0)$ & $0.0 \%(0)$ & $0.0 \%(0)$ & $0.0 \%(0)$ & -3.33 & 12 \\
\hline zemlać & $16.7 \%(2)$ & $8.3 \%(1)$ & $25.0 \%(3)$ & $5.0 \%(3)$ & $25.0 \%$ (3) & $0.0 \%(0)$ & $0.0 \%(0)$ & $0.0 \%(0)$ & $0.0 \%(0)$ & $0.0 \%(0)$ & $0.0 \%(0)$ & -2.67 & 12 \\
\hline złuszać & $16.7 \%(2)$ & $16.7 \%(2)$ & $8.3 \%(1)$ & $25.0 \%$ (3) & $16.7 \%(2)$ & $8.3 \%(1)$ & $0.0 \%(0)$ & $0.0 \%(0)$ & $8.3 \%(1)$ & $0.0 \%(0)$ & $0.0 \%(0)$ & -2.17 & 12 \\
\hline krąbać & $16.7 \%(2)$ & $16.7 \%(2)$ & $25.0 \%(3)$ & $8.3 \%(1)$ & $16.7 \%(2)$ & $0.0 \%(0)$ & $0.0 \%(0)$ & $0.0 \%(0)$ & $8.3 \%(1)$ & $8.3 \%(1)$ & $0.0 \%(0)$ & -2 & 12 \\
\hline wyteniać & $0.0 \%(0)$ & $16.7 \%(2)$ & $8.3 \%$ & $33.3 \%$ & $16.7 \%(2)$ & $8.3 \%$ & $8.3 \%$ (1) & $8.3 \%(1)$ & $0.0 \%(0)$ & & $0.0 \%(0)$ & -1.5 & 12 \\
\hline przenić & $0.0 \%(0)$ & $8.3 \%(1)$ & $25.0 \%(3)$ & $6.7 \%(2)$ & $8.3 \%(1)$ & $33.3 \%(4)$ & & & & & & -1.42 & 12 \\
\hline lipnić & $8.3 \%(1)$ & $8.3 \%(1)$ & $0.0 \%(0)$ & $5.0 \%(3)$ & $16.7 \%(2)$ & $33.3 \%(4)$ & $3 \%(1)$ & $0.0 \%(0)$ & $0.0 \%(0)$ & $0.0 \%(0)$ & $0.0 \%(0)$ & -1.33 & 12 \\
\hline goszyć & $0.0 \%(0)$ & $8.3 \%(1)$ & $25.0 \%(3)$ & $6.7 \%(2)$ & $8.3 \%(1)$ & $25.0 \%(3)$ & $0.0 \%(0)$ & $\%$ (1) & $3 \%(1)$ & $0.0 \%(0)$ & $0.0 \%(0)$ & -1.08 & 12 \\
\hline uzopyć & $0.0 \%(0)$ & $8.3 \%$ & $25.0^{\circ}$ & (1) & & $25.0 \%$ (3) & & & & & & -0.92 & 12 \\
\hline wnopić & $3 \%(1)$ & $16.7 \%(2)$ & $8.3 \%$ & $8.3 \%$ & $8.3 \%$ & $25.0 \%(3)$ & 8 & (0) & & & & -0.92 & 12 \\
\hline remić & $0.0 \%(0)$ & $8.3 \%(1)$ & $8.3 \%(1)$ & $8.3 \%(1)$ & $8.3 \%(1)$ & $66.7 \%(8)$ & $0.0 \%(0)$ & $0.0 \%(0)$ & $0.0 \%(0)$ & $0.0 \%(0)$ & $0.0 \%(0)$ & -0.83 & 12 \\
\hline mutnić & $8.3 \%(1)$ & $0.0 \%$ & $0.0 \%$ & $8.3 \%(1)$ & $25.0 \%$ & $50.0 \%$ & (0) & (1) & $6(0)$ & 0.0 & $0.0 \%(0)$ & -0.67 & 12 \\
\hline złuścić & $0.0 \%(0)$ & $25.0 \%$ & $0.0 \%$ & 33.3 & & & & & & & & -0.58 & 12 \\
\hline staleć & $16.7 \%(2)$ & $0.0 \%(0)$ & $0.0 \%(0)$ & $8.3 \%(1)$ & $8.3 \%(1)$ & $41.7 \%$ (5) & $8.3 \%$ (1) & $8.3 \%(1)$ & $0.0 \%(0)$ & $8.3 \%(1)$ & & -0.5 & 12 \\
\hline pejąć & $.0 \%(0)$ & $0.0 \%(0)$ & $8.3 \%(1)$ & $16.7 \%(2)$ & $8.3 \%(1)$ & $50.0 \%(6)$ & $8.3 \%(1)$ & $8.3 \%(1)$ & $0.0 \%(0)$ & 0.09 & $0.0 \%(0)$ & -0.42 & 12 \\
\hline spiszać & $0.0 \%(0)$ & $0.0 \%(0)$ & $16.7 \%(2)$ & $8.3 \%(1)$ & $16.7 \%(2)$ & $33.3 \%(4)$ & $16.7 \%(2)$ & $0.0 \%(0)$ & $0.0 \%(0)$ & $8.3 \%(1)$ & (0) & -0.33 & 12 \\
\hline kszenić & $0.0 \%(0)$ & & & & & & & & & & & -0.33 & 12 \\
\hline zomić & $0.0 \%(0)$ & $0.0 \%(0)$ & $0.0 \%(0)$ & $33.3 \%(4)$ & $8.3 \%(1)$ & $41.7 \%(5)$ & $0.0 \%(0)$ & $0.0 \%(0)$ & $0.0 \%(0)$ & $16.7 \%(2)$ & $0.0 \%$ & -0.08 & 12 \\
\hline pieczyć & 0 & & $0.0 \%(0)$ & & $16.7 \%(2)$ & & & & & & & 0.17 & 12 \\
\hline wdyrać & $16.7 \%(2)$ & $0.0 \%$ & $0.0 \%(0)$ & (2) & $0.0 \%$ & (2) & & & & & & 0.33 & 12 \\
\hline toleć & $0.0 \%(0)$ & $0.0 \%(0)$ & $0.0 \%(0)$ & $25.0 \%(3)$ & $0.0 \%(0)$ & $25.0 \%(3)$ & $33.3 \%(4)$ & $0.0 \%(0)$ & $\%(1)$ & $8.3 \%(1)$ & $0.0 \%(0)$ & 0.42 & 12 \\
\hline wiczyć & $0.0 \%(0)$ & $0.0 \%(0)$ & & & & & & & & & & 0.5 & 12 \\
\hline trużyć & $8.3 \%(1)$ & $0.0 \%(0)$ & $0.0 \%(0)$ & & & & & & & & & 0.5 & 12 \\
\hline szwyrać & $0.0 \%(0)$ & $0.0 \%(0)$ & $0.0 \%(0)$ & $0.0 \%(0)$ & $0.0 \%(0)$ & $58.3 \%(7)$ & $33.3 \%(4)$ & $0.0 \%(0)$ & $0.0 \%(0)$ & $8.3 \%(1)$ & & 0.67 & 12 \\
\hline kładnić & $8.3 \%(1)$ & $0.0 \%(0)$ & $0.0 \%(0)$ & $0.0 \%(0)$ & $8.3 \%(1)$ & $33.3 \%(4)$ & $16.7 \%(2)$ & $16.7 \%(2)$ & $8.3 \%$ (1) & $\%(0)$ & (1) & 0.67 & 12 \\
\hline pniczyć & $0.0 \%(0)$ & $3.3 \%(1)$ & $0.0 \%(0)$ & $3 \%(1)$ & $8.3 \%(1)$ & $16.7 \%(2)$ & $16.7 \%(2)$ & $0.0 \%(0)$ & $25.0 \%(3)$ & $6(0)$ & & 1.17 & 12 \\
\hline skopiać & $0.0 \%(0)$ & $0.0 \%$ & & & & & & & & & & 1.17 & 12 \\
\hline wpełać & $0.0 \%(0)$ & $0.0 \%(0)$ & $0.0 \%(0)$ & $0.0 \%(0)$ & $8.3 \%(1)$ & $16.7 \%(2)$ & $16.7 \%(2)$ & $25.0 \%$ (3) & $16.7 \%(2)$ & $8.3 \%$ (1) & $8.3 \%(1)$ & 1.83 & 12 \\
\hline wtężyć & $3 \%(1)$ & $0.0 \%(0)$ & $0.0 \%(0)$ & $0.0 \%(0)$ & $0.0 \%(0)$ & (0) & $33.3 \%$ (4) & $8.3 \%(1)$ & $25.0 \%$ (3) & $8.3 \%(1)$ & $16.7 \%(2)$ & 2 & 12 \\
\hline pększać & $0.0 \%(0)$ & & & & & & & & & & & 2.08 & 12 \\
\hline & $0.0 \%(0)$ & $0.0 \%(0)$ & $0.0 \%(0)$ & $0.0 \%(0)$ & $8.3 \%(1)$ & $16.7 \%(2)$ & $8.3 \%(1)$ & $25.0 \%(3)$ & $8.3 \%(1)$ & $25.0 \%(3)$ & $8.3 \%(1)$ & 2.17 & 12 \\
\hline składzić & $0.0 \%(0)$ & $0.0 \%(0)$ & $0.0 \%(0)$ & $8.3 \%(1)$ & $0.0 \%(0)$ & $16.7 \%(2)$ & $8.3 \%(1)$ & $8.3 \%(1)$ & $0.0 \%(0)$ & $41.7 \%(5)$ & $16.7 \%(2)$ & 2.58 & 12 \\
\hline namiecić & $0.0 \%(0)$ & $0.0 \%(0)$ & $0.0 \%(0)$ & $0.0 \%(0)$ & $0.0 \%(0)$ & $0.0 \%(0)$ & $25.0 \%$ (3) & $8.3 \%$ (1) & $50.0 \%(6)$ & $0.0 \%(0)$ & $16.7 \%(2)$ & 2.75 & 12 \\
\hline & $0.0 \%(0)$ & $8.3 \%(1)$ & $0.0 \%(0)$ & $0.0 \%(0)$ & $0.0 \%(0)$ & $0.0 \%(0)$ & $8.3 \%(1)$ & $16.7 \%(2)$ & $16.7 \%(2)$ & $16.7 \%(2)$ & $33.3 \%(4)$ & 2.92 & 12 \\
\hline & $0.0 \%(0)$ & $0.0 \%(0)$ & $0.0 \%(0)$ & $0.0 \%(0)$ & $0.0 \%(0)$ & $0.0 \%(0)$ & $0.0 \%(0)$ & $16.7 \%(2)$ & $25.0 \%(3)$ & $33.3 \%(4)$ & $25.0 \%$ (3) & 3.67 & 12 \\
\hline zwięczać & $0.0 \%(0)$ & $0.0 \%(0)$ & $0.0 \%(0)$ & $0.0 \%(0)$ & $0.0 \%(0)$ & $8.3 \%(1)$ & $8.3 \%$ (1) & $8.3 \%(1)$ & $8.3 \%(1)$ & $8.3 \%(1)$ & $58.3 \%$ (7) & 3.75 & 12 \\
\hline
\end{tabular}




\subsection{Experiment 6b}

Participants: 55 students of various Polish universities participated in the experiment online.

\subsubsection{Materials}

The training sentences and test materials were the same as for experiment $6 \mathrm{a}$ except that the novel verbs were changed. The four novel verbs were: pętrzyć, nabrzeć, zemlać, krąać, chosen after the norming study as suggesting "increase" or "decrease" (the ratings of the four words were 2.92, 3.62, -2.67, -2.00 respectively, see Table 3.13). As in Experiments 3, 4 and 5 , the absence of syntactic cues was ensured and all target nouns appeared in the same Dative case (which resulted in some sentences sounding a little unusual, albeit still grammatical).

\subsubsection{Procedure}

The task was the same as in the previous experiments. It was administered online, participants were recruited from student internet fora and volunteered to participate. There were four versions of the experiment equally distributed among the participants.

\subsubsection{Results}

One person's data was removed due to accepting everything as a 'guess'. Out of the remaining 54 participants, 10 expressed awareness of the regularity or reported potentially helpful observation, hence were classified as the "aware" group. The remaining participants were classified as the "unaware" group. As in the previous experiments, the analysis was performed on means corrected for small number of responses (unless otherwise stated).

The mean endorsement rates for the aware and unaware participants are shown in Table 3.14 below. 
Table 3.14. Mean endorsement rates (standard error in parentheses)

\begin{tabular}{|l|l|l|l|}
\hline & NG & NU & OG \\
\hline Aware $(\mathrm{n}=10)$ & $.49(.060)$ & $.40 \quad(.055)$ & $.70(.057)$ \\
\hline Unaware $(\mathrm{n}=44)$ & $.48(.028)$ & $.41(.031)$ & $.65(.025)$ \\
\hline
\end{tabular}

$\mathrm{NG}=$ New Grammatical, NU = New Ungrammatical, $\mathrm{OG}=$ Old Grammatical

An ANOVA on these data revealed that among the aware group the higher erroneous endorsement of word pairs which followed the selectional preference pattern (NG) than those that did not (NU) approached significance, $F(1,7)=5.05, p<0.5, \eta^{2}=0.42$. Accuracy for correct endorsement of the Old Grammatical (OG) items was significantly above chance on a single sample t-test: $\mathrm{t}(9)=3.52, \mathrm{p}<0.01$.

Crucially, the 44 unaware participants showed a significant difference between their endorsement of the NG and NU items, $F(1,40)=8.13, p<0.01, \eta^{2}=0.17$. Memory for the OG items was also significantly above chance, $\mathrm{t}(43)=5.75, \mathrm{p}<0.001$.

\section{Analysis by noun type}

An analysis by noun type (abstract or concrete) was carried out as in the previous experiments. The results are shown in Figure 3.23. 


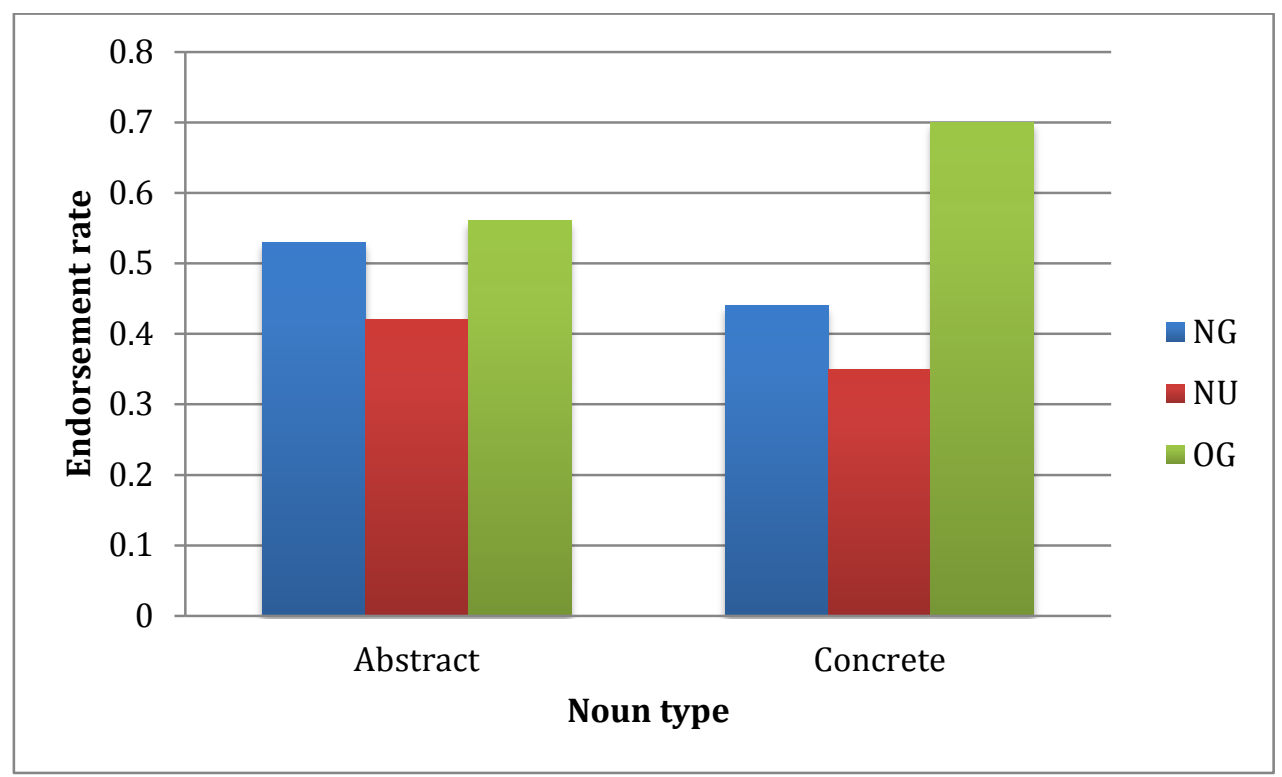

Figure 3.23. Endorsement rates for abstract and concrete collocates among the unaware participants.

An ANOVA was performed on the mean endorsement rates in the NG and NU conditions with grammaticality and noun type as within-subjects factors, and list as a between-subjects factor. There was a main effect of collocate type, $F(1,40)=10.88, p<0.01, \eta^{2}=0.21$, as the new stimuli with abstract nouns were endorsed more highly than with concrete nouns. There was also a main effect of grammaticality $F(1,40)=8.13 p<0.001, \eta^{2}=0.17$, but no interaction between collocate type and grammaticality, $\mathrm{F}<1.0$. Finally, for the OG condition, endorsement rates were higher for the concrete items than the abstract, $F(1,43)=9.61, p<$ $0.01, \eta^{2}=0.19$.

\section{Analysis of source judgements}

Among the 'unaware' participants, the source knowledge attributions were evenly distributed, as shown in Table 3.15 below. 
Table 3.15. Source proportions

\begin{tabular}{|l|l|l|l|}
\hline \multicolumn{2}{|l|}{ Source proportions } & & \\
\hline & NG & NU & OG \\
\hline guess & 0.295 & 0.334 & 0.241 \\
\hline intuition & 0.382 & 0.386 & 0.395 \\
\hline memory & 0.322 & 0.280 & 0.364 \\
\hline
\end{tabular}

Figure 3.24 shows the endorsement rates by source. Although numerically NG endorsement is higher than NU in all categories, the difference is only significant for 'guess': $F(1,31)=$ 9.03, $\mathrm{p}<0.01, \eta^{2}=0.23$. For 'intuition': $\mathrm{F}(1,34)=1.89, \mathrm{p}=0.18$; for memory: $\mathrm{F}(1,7)=$ $1.23, \mathrm{p}=0.28$.

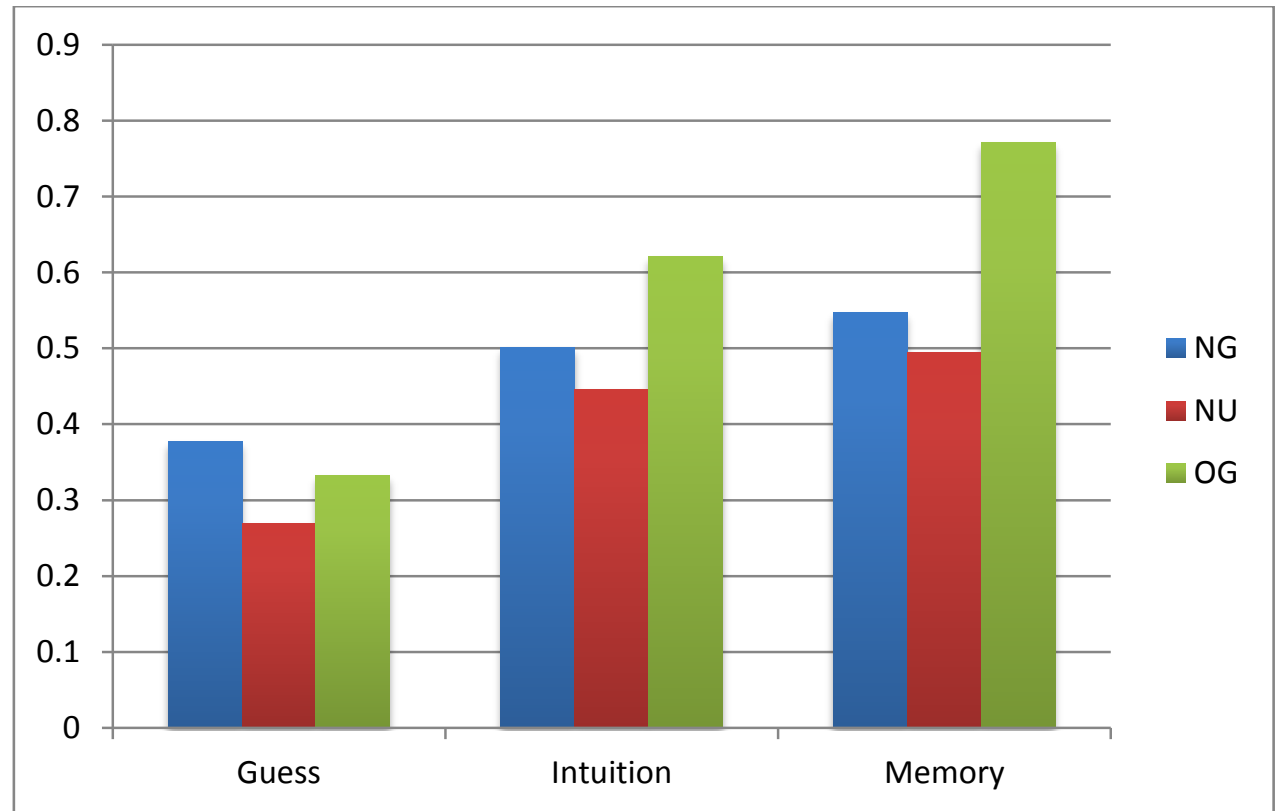

Figure 3.24. Endorsement rates by source, unaware participants. 
Analogously to the previous experiments the issue of whether participants used any potential knowledge strategically was first examined by comparing source judgements for endorsements and rejections of NG and NU items. For this reason, again, the guess-intuitionmemory categories were converted into a scale with values of 1,2 and 3 . The results of this analysis are shown in Figure 3.25.

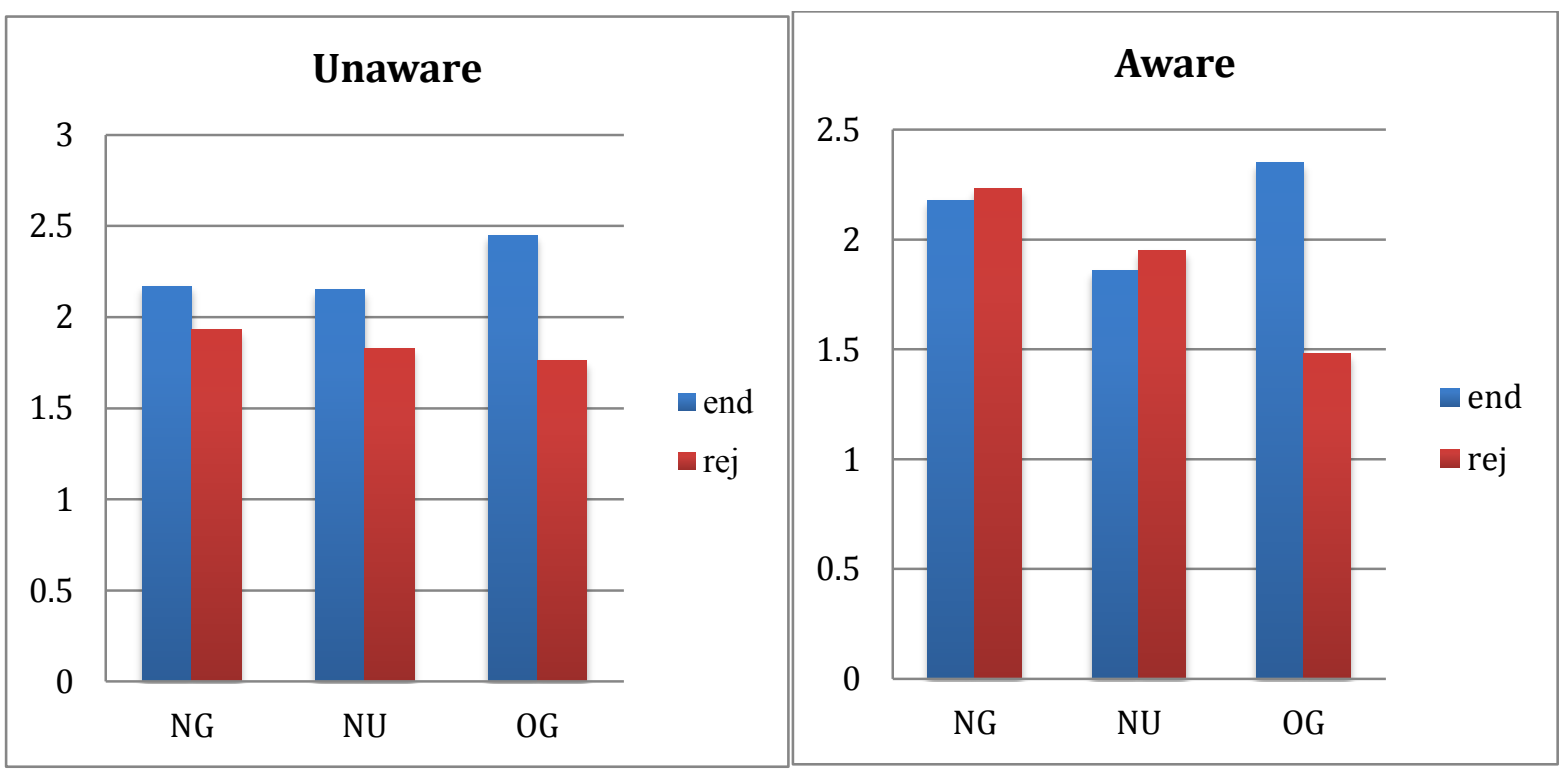

Figure 3.25. Mean converted scale for endorsements and rejections, for a) unaware, b) aware group.

An ANOVA was performed on the unaware participants' data on the mean confidence rates in the NG and NU conditions with grammaticality and endorsement/rejection as withinsubjects factors, and list as a between-subjects factor. There was a main effect of endorsement/rejection, $F(1,38)=9.62, \mathrm{p}<0.01, \eta^{2}=0.2$. There was no interaction between endorsement/rejection and grammaticality, $\mathrm{F}<1.0$. Conscious knowledge was hypothesised to be reflected by higher confidence for rejecting NU items than NG items. A numerically opposite pattern was found (though not statistically significant, $F(1,42)=1.54, p>0.1, \eta^{2}=$ 0.035), inviting a potential conclusion that this group of participants were rather unlikely to have strategically used conscious knowledge of grammaticality.

Again, strategic use of conscious knowledge was also hypothesised to lead to a correlation between the difference in rejection confidence between NU and NG items and the 
learning effect (NG-NU endorsement difference). Scatter plots for the unaware and aware groups are shown in Figure 3.26.
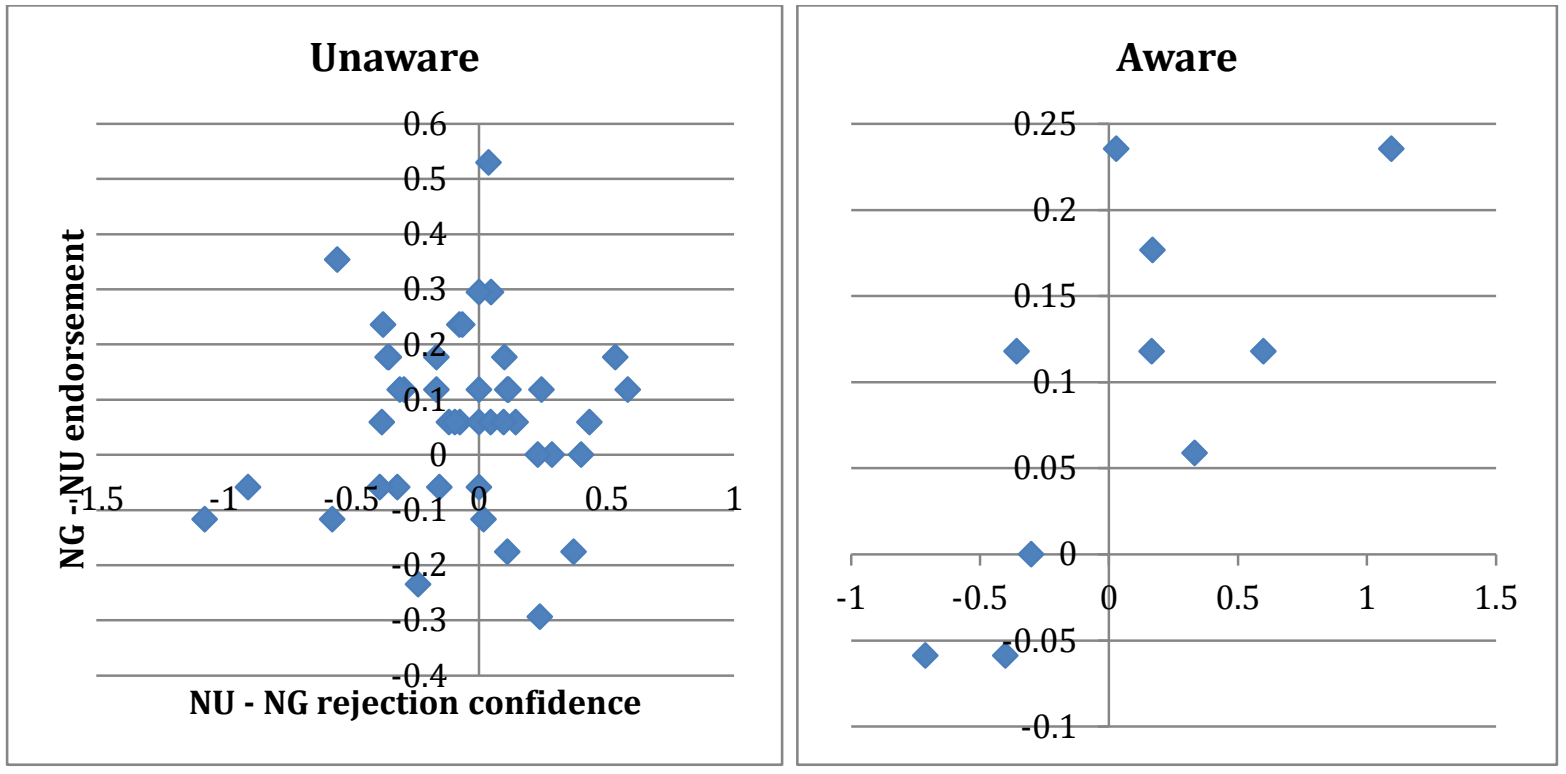

Figure 3.26. Confidence difference for NU-NG rejections and learning effect (NG-NU endorsement difference with Bayesian correction), for a) unaware, b) aware group.

The unaware group showed no correlation, $\mathrm{r}(42)=0.045$. On the other hand, the aware group showed a significant correlation $\mathrm{r}(8)=0.71, \mathrm{p}<0.05$.

\subsubsection{Discussion of Polish experiments 6a and $6 \mathrm{~b}$}

The two experiments $6 \mathrm{a}$ and $6 \mathrm{~b}$ on Polish native speakers confirmed that the learning which is revealed in this procedure does not arise simply due to participants' recognising at some level, either conscious or unconscious, the existing English equivalents of the target words, with their semantic selection patterns. If that were the case, Polish speakers would not have shown learning effects on the same material as English speakers, which they did in experiment $6 b$.

The apparent absence of any learning in experiment $6 \mathrm{a}$ is interesting, as the material was exactly the same as $6 \mathrm{~b}$, with only different target word forms. The precise conditions under which semantic implicit learning can be obtained is an interesting question worth 
future investigation, especially in the light of theories of semantic knowledge. For example, it is not clear how purely association-based models would account for this finding (see 3.8.5 Discussion below).

The factor hypothesised here to be the reason for the non-significant effect of grammaticality in experiment 6 a was the arbitrariness of word forms. Even though the change of effect following the norming study may suggest this was indeed the case, a definite conclusion cannot be reached at this stage. There could still be other factors involved, such as wordlikeness (e.g., Bailey \& Hahn, 2001), or word memorability, given that the aware group (though not the unaware group) reported low recognition of Old Grammatical (OG) phrases with abstract nouns. Future experiments controlling these factors may further our understanding of what drives semantic implicit learning processes, but what can be stated at this stage is that this is another example of prior lexical knowledge exerting a top-down effect on learning (cf. Brockmole et al, 2008).

\subsection{General discussion of false memory experiments}

Overall, the series of seven experiments presented in this chapter confirm the hypothesis that people implicitly generalise the meaning of nouns collocating with novel verbs. The method proposed here is based on the false memory phenomenon, and the effect is assumed to occur automatically and unconsciously in unaware participants. The detailed analysis examining the correlation between the learning effect and confidence ratings revealed that the vast majority of participants did not intentionally and strategically use any explicit knowledge of the semantic preference rule that they may have possessed. This was determined by the lack of significant correlations between the learning effect and the NU-NG rejection confidence difference among the unaware group. Furthermore, Experiment 5, the explicit version of Experiment 4, verified the methodology by showing that the task does not naturally incline people to use their knowledge strategically, even if they are able to report that knowledge. It turned out that even in this condition some people behaved like the unaware groups and some like the aware groups in other experiments. The ones who resembled members of the other aware groups, and clearly used their knowledge strategically, also performed perfectly on the subsequent post-test. On the other hand, those who, despite being provided with the semantic 
regularity explicitly, still performed akin to the unaware groups (as can be seen from the scatter plot in Figure 3.21), did not perform perfectly on the post-test. It can be concluded, therefore, that the false memory paradigm can be used as an indirect measure of implicit knowledge provided that confidence judgements are used to exclude cases where explicit knowledge has been deployed intentionally.

The findings from this series of experiments confirmed that people form semantic generalisations without awareness. The question which now arises is: how does this happen? One plausible approach is along the lines of the 'Multiple Trace' memory model proposed by Hintzman (1986). It is suggested that every experience forms a separate trace in memory. For example, here the learners see for example the novel word POWTER followed by the collocate the prominence, which results in the formation of a trace comprising the form of the novel word, and the form and meaning of the collocate. In the course of the training phase a participant accumulates more experiences and encounters, for example, POWTER the role and POWTER the status. After such experiences, on seeing the word POWTER, the response from memory would be the average of all memory traces in which that word was encoded essentially a prototype representation resulting from all the encountered specific instances. As the model relies on featural representations of meaning it can be assumed that averaging over traces results in a prototype representation. In the current case, an average collocate of POWTER is 'something abstract'. In the test phase, on seeing POWTER with a new word the phrase is projected to memory and resonates with traces according to its similarity to them, producing an "echo" with a strength that is related to the degree of match. When a new abstract word, e.g. esteem, is presented in connection with POWTER, because it has semantic features that are similar to the ones associated with POWTER in memory, the echo from memory will be relatively strong compared to, for example, when POWTER occurs with a word like sugar. Such an approach predicts that similarity between test items and particular training items will increase the echo strength, which would explain why the learning effect appeared to be stronger in experiment 3 than experiment 4 (where heterogeneous exemplars of each category were used). Note also that the conscious feeling of familiarity does not entail that the structural knowledge leading to this intuition (here that POWTER collocates with abstract words) is conscious.

The Multiple Trace model, however, is at odds with mainstream views on false memory. On the one hand, there are models based on feature semantics, (e.g., Eich, 1982), which, similarly to Hintzman, account for the false memory phenomenon in terms of feature 
overlap, and predict an important role for across-item semantic relations in the generation of false memories (e.g. Brainerd \& Reyna's 2005 fuzzy-trace theory). On the other hand, there are numerous accounts based on the view of semantic knowledge as a network of associations, or word co-occurrences. On this view, apple and pear are not related because they share features, but rather because they occur in similar contexts. Also, apple and pick are associates, not because of feature overlap, but because of their patterns of co-occurrence. Examples of this view of semantic space are presented by Nelson, Bennet, and Leibert (1997), Lund and Burgess (1996), McEvoy, Nelson \& Komatsu (1999), Roediger, Balota et al. (2001), and Roediger, Watson, McDermott, \& Gallo (2001). Both feature- and association-based models of semantics account for false memory phenomena in terms of spreading activation, the former along the overlapping features, the latter along the connections based on occurrences.

There are numerous arguments in support of the associationist views of false memory, and indeed it is the predominantly favoured view in the field. The central argument for it is the finding that the strongest predictor of false memories is backward associative strength from the presented training item back to the unpresented test lure (Roediger, Watson et al., 2001, Buchan, Brown, Cabeza \& Maison, 1999). Further support for the associationist view comes from developmental and dementia studies. For example, Bjorklund and Jacobs (1985) demonstrated facilitation effects in associated lists in both young and older participants ( 8 to 20 years old). However, categorical lists stimulated facilitation only among the older group, suggesting the primacy of associative semantic relations, with the ability to make use of category knowledge developing over time. Also, category priming was shown already to be impaired in the early stages of Alzheimer's disease, while associative priming remained comparable to healthy age-matched controls (Glosser et al., 1998, Ober, Shenaut, \& Reed, 1995), which further reinforces the idea that associative semantics can be more rudimentary.

At odds with the current experiments is the finding reported in Hutchison and Balota (2005), who concluded that thematic consistency is irrelevant for false memory. Using a DRM procedure, they compared lists of 6 words all suggesting one nonrepresented lure (e.g., snooze, wake, bedroom, slumber. . ., for SLEEP) and 6 words that converged onto a different meaning of a homograph (e.g., stumble, season, trip, autumn. . . , for FALL). Associative strength from the list items to the critical target was equated across the two types of lists. Differences in recall were only marked for veridical, but not false memory, suggesting that the latter does not rely on the formation of meaningful representations across items. 
It is difficult to distinguish between feature-based and association-based theories at this stage. The experiments rely on participants' already formed lexicons. If the lexicon is based on associations, then the effects reported here would have to be explained on that basis. It would indicate that, having seen POWTER the status people activate the words which have been associated with status by virtue of having encountered them in similar contexts. Future research with careful control for backward association strength between test words and training words, as well as an analysis of people's semantic similarity ratings, could shed more light on this major debate in linguistics - what the structure is of our semantic knowledge.

The findings reported in this chapter are relevant to the debate regarding the role of attention to the relevant meaning in an implicit learning task. Here, the participants were not directed to attend to the abstract/concrete distinction. This feature must have been implicitly activated, and it may be claimed that it became activated as an inherent feature of a given noun (cf. Williams, 2005; Leung \& Williams, 2011). This raises the question of what is the minimum that has to be "noticed" in order for implicit learning to occur. It appears that, whilst noticing the relevant form is presumably necessary, noticing the relevant semantic distinction is not. This issue will be revisited in the next chapter.

In all, the chapter presents a collection of experiments using an improved methodology for addressing semantic implicit learning over that reported in Chapter 2. However, can the existence of the phenomenon be proved at this stage? Sceptics might still argue that people are intentionally ${ }^{14}$ matching the test items to the exemplars they remember. In other words, the effect reported here occurs at the retrieval stage, and does not pertain to the learning process as such - which is an objection raised in the past to the classical implicit learning demonstrations (see Chapter 1). In order to establish that the phenomenon is not merely a retrieval effect, yet another diagnostic test of implicitness should be applied, namely automaticity. This will be addressed in the following chapter.

\subsection{Chapter summary}

This chapter contains 7 experiments based on a false memory task addressing semantic implicit learning in language.

\footnotetext{
${ }^{14}$ This is the critical distinction between these and the reaction-time based experiments in Chapter 4 .
} 
In Experiment 2, participants were first exposed to sentences, some of which contained one of four novel verbs. In a subsequent surprise test participants saw verb-noun pairs, and were asked whether these pairs had appeared in the training. Although all of the words presented in the test had appeared in the training individually, most of them did not occur together. Crucially, half of the new combinations followed the pattern of semantic preferences from training, the other half violated it. Should semantic implicit learning take place, participants were predicted to erroneously judge as familiar those that followed the pattern significantly more often than those that did not. This effect was found in two variants, experiments $2 \mathrm{a}$ and $2 \mathrm{~b}$, the latter maintaining a closer syntactic similarity between training and test items than the former.

Experiment 3 assured external validity of the laboratory-based findings by replicating the first laboratory experiment (with minor changes on a small number of stimuli) in an internet-based version using a large, and highly heterogeneous, participant sample.

Experiment 4 examined whether the false memory effect could still be obtained when the nouns in the test phase were semantically dissimilar to those that occurred with the nonwords in training, showing broad generalisation of the abstract/concrete regularity. Such an effect was obtained, although the effect size turned out to be much smaller than in the previous experiments. This may suggest that semantic similarity between training and test items plays a role in the false memory effect.

Experiment 5 showed that explicitly instructed participants did not differ from implicit groups in their performance on the false-memory task, suggesting that even when participants have conscious knowledge they do not use it strategically when judging familiarity, confirming that false memory effects provide a good indirect measure of implicit learning.

Experiment $6 a$ and $6 b$ addressed the issue whether participants could have critically relied on the recognition of real English equivalents for target words. The materials were Polish translations of the sentences used in Experiment 2. Interestingly, although Experiment $6 \mathrm{~b}$ showed evidence of implicit learning, this only happened after target words were normed for how suggestive they were of increasing/decreasing. Experiment $6 a$, which relied on the same materials as $6 \mathrm{~b}$ but with different target words, failed to induce implicit learning effects. This observation raised questions about the dependency of implicit learning on prior semantic knowledge. 
The results were then discussed in light of theoretical models of lexicon and false memory as well as methodological issues concerning implicit learning research. It is argued that despite the fact that explicit knowledge can influence judgements in this task, strategic use of such knowledge is visibla as a correlation between the learning effect and confidence, and, importantly, only concerns the most highly aware participants. Overall, therefore, the false memory task offers a good way to assess implicit knowledge. 


\section{Chapter 4}

\section{Semantic Implicit Learning and Automaticity}

\subsection{Introduction}

A trademark of implicit processes is automaticity. While explicit knowledge can guide intentional behaviour, implicit knowledge acts independently of conscious monitoring, in other words automatically (Cleeremans \& Jimenes, 2002), Dienes \& Perner, 1999). In the field of SLA, automaticity has often been assumed to be reflected in fluency, which, in turn, has been assumed to be an index of acquisition as opposed to learning (cf. Krashen, 1981, Monitor Hypothesis). Fluency and automaticity have usually been addressed by imposing a time pressure for response. For example, Rod Ellis (2005) performed a principal components factor analysis on five different language tests, showing that speeded grammaticality judgement, oral imitation and oral narrative loaded on a common factor, while the untimed grammaticality judgement and a metalinguistic knowledge test loaded on another. Ellis proposed that the factor that distinguished these two groups of tasks was time pressure and that speed of response can distinguish between implicit and explicit processing. His finding was later disputed on methodological grounds (Isemonger, 2007). Furthermore, as illustrated in Chapter 1, section 1.8, explicit knowledge may become automatic through proceduralisation, suggesting that not all fast and fluent behaviour necessarily 
results from implicit learning. Operationalisation of implicit learning through automaticity therefore requires further postulates and delineation of the specific role of awareness.

As Tzelgov (1997) points out, in an automatic process participants can still be aware of the stimuli that are being processed (c.f. Schmidt's "noticing" hypothesis), but the process runs without being consciously monitored. Therefore, automaticity coupled with measures of awareness could offer a useful diagnostic of implicitness. Tzelgov proposed that a process can be said to run independently of conscious monitoring if it can be shown to operate when it is not part of the task requirement. Otherwise the process is "intentional", in which case it is difficult, if not impossible, to assess whether or not it runs implicitly. The most well-known demonstration of automatic processing is that in the "Stroop task" (Stroop, 1935), where people are shown to be slower to name the colour in which a word is printed if the word's meaning denotes a different colour, (e.g., Blue, Purple, Red, Green, Purple, Green), than when the two are congruent (e.g., Green, Red, Blue, Purple, Blue, Purple). The task only requires participants to name the colour, yet the fact that the word meaning interferes with it anyway, without participant's intention for it to do so - suggests that meaning activation is automatic.

To demonstrate implicit learning, automaticity needs to be assured both during the acquisition of knowledge and its retrieval. During retrieval, participants may consciously use explicit knowledge to help them perform the task, in which case their behaviour becomes controlled. Overall, in a fully automatic task, such knowledge should be neither required nor even beneficial to the task intentionally performed. The present experiments adopted this approach, whilst supplementing it with verbal report as a means of assessing awareness of the target semantic preference system.

An example of a fully automatic task in implicit learning research is that used by Vinter \& Perruchet $(1999,2000)$. They demonstrated that adults and children nondeliberately modified their natural drawing behaviour following a specially designed practice. The procedure was based on the "start-rotation principle" in drawing (Van Sommers, 1984), which postulates that the direction of drawing a closed geometrical figure depends on a starting point. Using a circle as an example, if the starting point lies above a virtual axis between 11 o'clock and 5 o'clock on a clockface, people are naturally inclined to draw anticlockwise, whereas if the starting position is set on the lower circumference - they draw clockwise. Participants in Vinter \& Perruchet's study were divided into three groups: the incongruent, congruent and baseline. Their task was to draw over figure shapes. In the first two groups they were given both the starting point and the direction of drawing (which in the 
incongruent group violated the natural inclination in $80 \%$ of cases, while in the congruent group reinforced it in $80 \%$ of cases). The free baseline group could draw as they chose. It turned out that the participants inverted the direction of rotation associated with a starting location after being trained to respect the reverse rotation. They did that without being explicitly aware of the manipulation (as revealed by a post-experiment task). In this experiment participants' attention is drawn to speed and accuracy, while learning is measured by a different parameter, namely the drawing direction, which makes the emerging effect a clear reflection of automatic processing.

This type of modification of behaviour can be interpreted as a form of priming people are not aware that their behaviour has changed because of their prior experience. Importantly, though, they are conscious at the point of drawing in which direction they do it. What they are not conscious of is the fact that their behaviour was modified and how. After training, it feels easier for them to draw figures in a particular direction. Clearly, therefore unconscious processing (structural knowledge) can have conscious effects (judgement knowledge) (Dienes \& Scott, 2005).

The experiments presented in this chapter use a modification of the standard priming procedure in psycholinguistics to address the question of whether semantic implicit knowledge is expressed automatically in such a way as to affect real-time processing. Such a finding would demonstrate the relevance and presence of this phenomenon in natural language use. Is it possible to replicate the results obtained using a false memory paradigm (in Chapter 3) using a different method, measuring the timing of participants' responses and demonstrating both automaticity and implicitness (unawareness)?

The five experiments reported below involve progressively improved attempts at revealing implicit learning through on-line tasks. However, it must be acknowledged that, although in the initial two pilot studies, as well as Experiment 7 and 8, the knowledge of the target system is not part of the task, explicit knowledge of this system would nevertheless be beneficial for performance and would affect the measure of learning. This issue will be discussed further in the relevant sections. However, the final experiment, Experiment 9, presents a procedure which could be regarded as fulfilling the above described criteria for automaticity: the target regularity is neither part of the task, nor beneficial for it.

The first pilot study, presented in the following section, tested whether knowledge implicitly gained through the same training as in Chapter 3 could exert its influence automatically. 


\subsection{Pilot 1}

Participants: 21 students from the University of Cambridge participated in the study.

\subsubsection{Materials}

To allow close comparisons between automatic measures of implicit learning and the false memory paradigm discussed in Chapter 3, the training materials for this experiment were identical to those used in Experiment 2a. The test items included all the phrases from Experiment 2a (containing the nouns which did appear at some point during the training) plus 16 new 'filler phrases'. The 'filler phrases' contained novel verbs with new nouns, which had not appeared in training. Just like the test phrases, the fillers were composed either grammatically, or ungrammatically: that is, the novel verb was either followed by the same abstract/concrete noun type as it had appeared with in the training (grammatical filler), or by the other noun type (ungrammatical filler). There were four versions of the experiment, first the nouns were rotated between the grammatical and ungrammatical ones, and then the nonwords were rotated in relation to the increase/decrease distinction as well as the abstract/concrete.

\subsubsection{Procedure}

Training followed the same procedure as Experiment 2a. Test phrases were presented one word at a time (e.g. powter-importance, mouten-nitrogen). Primes (verbs) were presented for $250 \mathrm{~ms}$ with no interval before the target, following the suggestion that at this brief stimulus onset asynchrony (SOA) priming effects are automatic and not affected by conscious expectancies, i.e. controlled processing (Neely, 1977). Participants were told they would see two words, one appearing after the other. Their task was to indicate whether they had seen the second word in each pair in Part 1 of the experiment (the training). By making this decision, as opposed to whether they had seen the two words together, the measure of learning (response times to correct and incorrect new pairings) is not related to the potentially explicit knowledge of the system. Such knowledge is neither part of the task nor beneficial for it, in 
the spirit of Tzelgov's (1997) argumentation. Participants were asked to respond as quickly and as accurately as possible using a Cedrus response pad. The stimuli were displayed on a computer screen using SuperLab $4.0^{\circ}$. Finally, the same post-experiment test and questionnaire was administered as in Experiment 2a. It was predicted that, if semantic knowledge gained in implicit learning tasks did indeed exert its influence automatically, the following should be observed:

1) Shorter RTs for grammatical than ungrammatical stimuli, for both test and filler items;

2) Higher endorsement rates for grammatical than ungrammatical stimuli, for both test and filler items (replicating the off-line measures from the experiments reported in Chapter 3).

\subsubsection{Results}

In the post-experiment questionnaire, 9 out of 21 participants expressed potentially relevant explicit knowledge and were classified as the 'aware' participants. The remaining 12 were classified as 'unaware'. An ANOVA was performed on mean reaction times in the 'new grammatical' (NG) and 'new ungrammatical' (NU) conditions in which Condition (NG or NU) was a within-subjects factor and participant group a between-subjects factor. An analogous analysis was performed on reaction times for the two conditions for the new filler items. The same type of analysis was performed on the endorsement rates in the NG and NU, and the endorsement rates in the grammatical and ungrammatical fillers. None of the predictions about the automaticity of unconscious semantic knowledge gave rise to significant effects:

1) For the aware participants' there were no significant differences in reaction times between the NG and NU test items: $F(1,5)<1.0$, nor between the 'grammatical' and 'ungrammatical' new filler items: $F(1,5)=2.80, p=0.15, \eta^{2}=0.36$. A similar pattern of results, with no significant differences in reaction times, was shown for the 'unaware' participants. An ANOVA on NG and NU revealed $\mathrm{F}(1,8)<1.0$, and on filler items also $F(1,5)<1.0$. The 'unaware' group's mean reaction times are depicted in Fig. 4.1a. 
2) The endorsement rates, just like in the experiments in Chapter 3, were corrected for small number of responses, with the correction calculated as (number of correct responses +0.5$) /($ total number of responses +1$)$ (Dienes, 2012). Surprisingly, in this paradigm, the pattern of results which was rather stable in the Chapter 3 experiments did not emerge. The 'aware' group did not endorse more NG than NU test items: $F(1,5)=2.33, p=0.19, \eta^{2}=0.318$, nor did they endorse more grammatical than ungrammatical filler items: $F(1,5)=4.65, p=0.08, \eta^{2}=0.48$. The unaware group also showed no significance differences $(\mathrm{F}(1,8)<1.0)$ in these two tests. The unaware group's endorsement rates in all conditions are depicted in Fig. 4.1b.

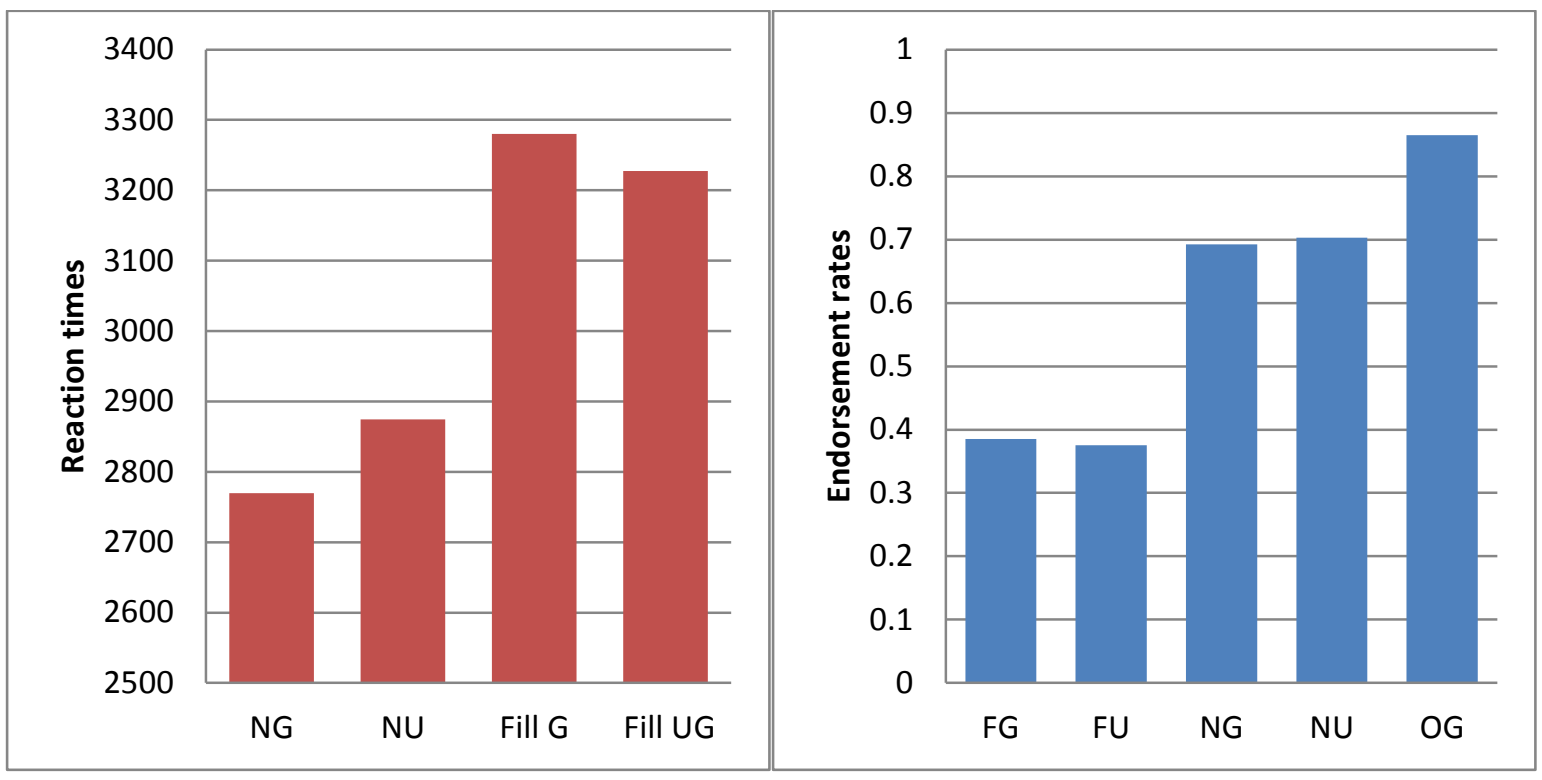

Figure 4.1 Unaware participants' a) mean reaction times, b) mean endorsement rates per condition. Note: $\mathrm{FG}=$ Grammatical Filler item, FU = Ungrammatical Filler item.

\subsubsection{Discussion}

Contrary to the initial predictions, participants' reaction times in the grammatical and ungrammatical conditions did not reveal evidence for semantic implicit knowledge, suggesting that it may not exert its influence automatically. A particularly surprising result emerged in the endorsement rates for the grammatical and ungrammatical conditions, showing no difference between them. This is in stark contrast to Experiment 2a in Chapter 3, 
where a significant effect emerged while the same test stimuli were used. What could be responsible for the discrepancy between these two experiments?

One possible explanation is that in the current pilot study participants may have been ignoring the primes. The task only pertains to the noun. It is possible to go through the test phase without even reading the primes.

An alternative explanation for the possible loss of the learning effect on endorsement could be due to this version being not faithful enough to Experiment 2a. Perhaps the inclusion of the 16 new filler words had a detrimental effect. Or perhaps the primes were presented too briefly to activate the relevant information. After all, Neely's (1977) demonstration of automaticity at a $250 \mathrm{~ms}$ SOA was for semantic priming from highly familiar words. Before drawing final conclusions, it is therefore worth analysing another pilot study, which is equivalent to this one in all aspects apart from the presence of the filler test items and the use of a longer prime exposure.

\subsection{Pilot 2}

Participants: 19 students from the University of Cambridge participated in the experiment.

\subsubsection{Materials}

Materials used in this study were identical to the Pilot 1 study above, with the exception of the exclusion of the filler items in the test.

\subsubsection{Procedure}

The study followed the same procedure as Pilot 1, but with primes presented for $750 \mathrm{~ms}$. Again, if implicitly learned semantic knowledge exerted its influence automatically, the unaware participants' reaction times to endorse the nouns in the NU trials should be slower than the reaction times for the nouns in the NG trials. It was also predicted that the same false-memory pattern of endorsement would occur as in Experiment 2a and the other 
experiments reported in Chapter 3, namely higher endorsement rates for the NG than the NU trials. Note that in this case, all nouns did appear in the test, so in principle all should be endorsed. NG and NU still refer to the new verb-noun mappings, where the noun follows a verb either in agreement with or in violation of its semantic preference pattern.

\subsubsection{Results}

In the final questionnaire 3 out of 19 participants made observations about certain words being appropriate for abstract/concrete nouns and were classified as the 'aware' participants. The remaining 16 remained 'unaware' of the regularity; many of them expressed surprise when informed about it.

There were too few aware people to carry out a separate analysis on them. However, the unaware group was the crucial one for the investigation of implicit learning. In this experiment, just like in Pilot 1, neither an ANOVA on reaction times in NG and NU $(F(1,12)$ $<1.0$ ), nor even on endorsement rates in NG and $\mathrm{NU}\left(\mathrm{F}(1,12)=1.87, \mathrm{p}=0.2, \eta^{2}=0.14\right)$ ) revealed a significant difference. Figure 4.2 depicts the unaware participants' a) reaction times, and b) endorsement rates.

Again, it appears that under the current speeded response conditions with a sequential presentation of verb-noun phrases, the false-memory effect from Chapter 3 disappears.

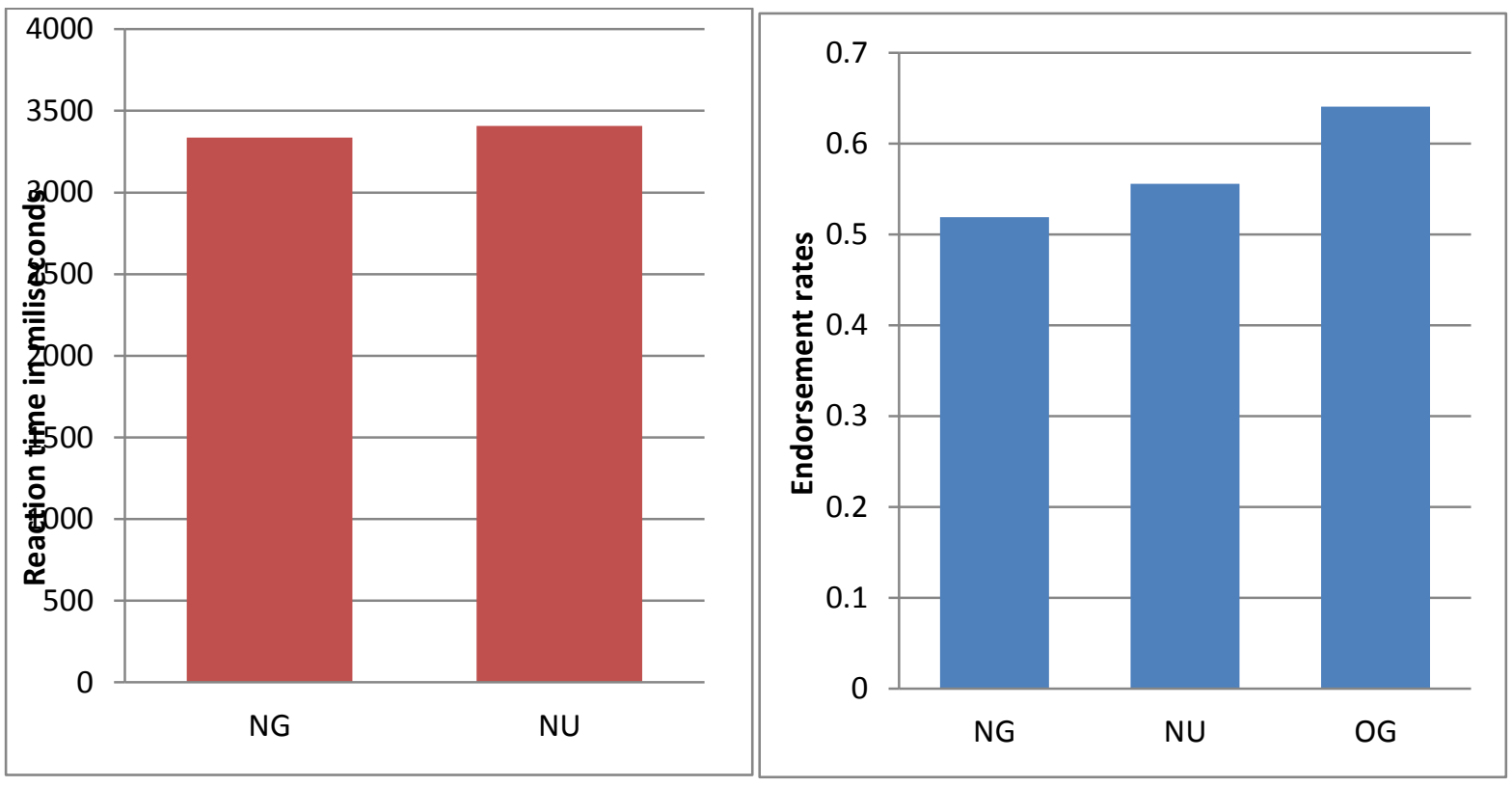

Figure 4.2. Unaware participants' a) endorsement reaction times, b) endorsement rates. 


\subsubsection{Discussion}

Neither in Pilot 1 nor Pilot 2 did the same false memory effect as in the Chapter 3 experiments emerge. There were also no differences in reaction times of endorsement in the NG and NU conditions.

A possible explanation for this discrepancy could be the mode of presentation. Seger (1998) presents a collection of priming and implicit learning studies demonstrating that the effects disappear with sequential presentation of stimuli, e.g. Goshen-Gottstein and Moscovitch (1995). She suggests that spatial and temporal contiguity plays a significant role in implicit learning. This in turn has to do with such factors as the principle of congruity (Schulman, 1974), transfer-appropriate processing or the encoding of specificity principle. Before considering those, however, it is worth analysing one additional factor.

A different explanation for the apparent discrepancy between these results and those reported in Chapter 3 could be insufficient depth of processing of the prime and target words. According to the Craik \& Lockhart (1972; Craik and Tulving 1975), processing based on perceptual features, such as orthography or phonemes, leads to a fragile memory trace prone to rapid decay. Deeper processing, such as semantic processing, results in a more durable memory trace. Although both Chapter 3's experiments and the current experiments involved the same training, shallower processing of test phrases in the current pilot studies may have hindered the effect. It is true that neither in the Chapter 3 experiments nor the ones here did the test task require deep semantic processing. Crucially, however, in Chapter 3 the test question was: "did you see these words next to each other?" while here it was "did you see the second word in part 1?" Separate presentation of the words not only makes any processing of the prime (verb) unnecessary, but may also hinder processing the two words as a single unit. It is the relationship between the two words that is of key importance in the experiment, therefore perhaps it would be optimal to process both at the same, deep semantic level. Furthermore, in the current study there is also time pressure for the response. Semantic features may take a different time course of activation (Yee et al., 2011), and it is not inconceivable for speed of response to interfere with the otherwise unhindered semantic processing taking place in standard, natural, unspeeded conditions, such as those in the Chapter 3 experiments.

In an attempt to increase the depth of processing of the otherwise meaningless verbs, it might be beneficial to introduce a semantic task to perform on them. In what follows, a task 
to indicate abstract/concrete is therefore introduced on the nouns, and an increase/decrease decision relating to the verbs. This does not undermine implicit, automatic processing, since, as mentioned above, participants can be aware of what they are processing, so long as they do not monitor it while performing the task.

A final modification to the procedure is the introduction of a continuous measure of the learning process so that participants do not even know when the test begins.

\subsection{Experiment 7}

Participants: 40 students of the University of Cambridge participated in the experiment. 17 were native speakers of English, other languages included: Chinese $(n=6)$, Dutch $(n=3)$, French $(n=1)$, German $(n=4)$, Hindi $(n=2)$, Lithuanian $(n=1)$, Korean $(n=2)$, Portuguese $(\mathrm{n}=1)$, Punjabi $(\mathrm{n}=2)$.

\subsubsection{Materials}

Materials were similar to those in Experiment $2 \mathrm{a}$ and $2 \mathrm{~b}$. The word fonell was changed to conell to avoid the similarity with funnel, which could have suggested an action involving some tangible substance. Long sentences were shortened in order to facilitate their accessibility in the word-by-word presentation procedure. Some sentences from Experiment 2a, especially those involving concrete nouns, were changed to new ones in order to allow the use of the definite article, which in this experiment appeared before all target nouns (to eliminate syntactic cues). All training and test items are included in Appendix E.

\subsubsection{Procedure}

The experiment was composed of two parts following an identical procedure. The training part contained 60 well-formed sentences, called 'grammatical' for the current purposes, where two verbs were always followed by abstract nouns, and the other two by concrete 
nouns. The testing part contained 76 sentences, in which 32 were critical, while the remainder simply provided further exposure to positive evidence. Some of the sentences from the training were repeated in the test in order to provide further exposure. They intervened between the critical, 'new grammatical'(NG) and 'new ungrammatical' (NU) test sentences. There were 8 critical sentences for each word, half of them presenting 'grammatical' contexts, the other half 'ungrammatical' ones.

Participants did not know that there were two separate stages in the experiment, although they did have a short break in between. The experimental task was explained in steps. First, it was demonstrated that the sentences were going to appear word by word. Then, the following instruction was presented:

In each sentence one of the verbs will be a novel word written in CAPITALS. For each sentence your task is to decide whether this novel word indicates roughly 'becoming less of' (-) or 'becoming more of' (+) in that sentence.

Participants were asked to use two edge buttons on a Cedrus ${ }^{\odot}$ response-pad to indicate their decisions and instructed to do so as quickly but as accurately as possible. The experiment was implemented in SuperLab Pro 4.0.

Participants were then informed that the sentences were going to appear in a slightly scrambled word order, with the verb always appearing at the beginning. However, the decision about whether it meant that something was increasing or decreasing was only required at the end of the sentence. The purpose of that decision was to induce deeper processing of the novel verb. There was no information provided about which words meant 'increase' and which 'decrease', nor whether the meanings were going to be stable. This was to assure that participants read the sentences carefully (especially the crucial nouns).

Participants were then informed that there was also going to be a task for them to perform during the sentence reading. One word was going to be presented in red and they were asked to indicate as quickly and as accurately as possible whether that word denoted something abstract or concrete. They were given no more than 3 seconds to perform that decision, in order to minimise any strategic thinking. If they made an error the target did not disappear and they had to correct their response. This measurement was taken on the noun, as it was the point at which violations of the system took place (a novel verb being matched with the wrong type of abstract/concrete noun). This is the earliest point at which it is 
possible to measure a potential effect of semantic expectations deriving from the novel verb. The abstract/concrete decision was followed by a recall prompt, where participants had to repeat to the microphone the phrase composed of the initial verb and the noun presented in red. Below, Figure 4.3 exemplifies the procedure on a 'grammatical' and 'ungrammatical' sentence, with the required tasks explained underneath each stage.

Note that in Experiment 2 in Chapter 3 the feature of "abstractness/concreteness" was not mentioned or required to perform the task and participants must have activated it as a byproduct in the course of doing the experiment. Here, this feature is explicitly mentioned in the instruction. This does not pose a challenge to the proposal that the experiment addresses automatic influence of unconscious knowledge. As argued in the Introduction to this chapter, the crucial factor is that the process runs without monitoring, by virtue of the target regularity not being a part of the task. Participants may be aware of what they are processing.

Here, it is the relationship between the novel verb and the type of noun which is the crucial target, and that is not mentioned upfront. Therefore, although, in Schmidt's (1993) terms, "noticing" of the relevant feature is aided by the task instruction, this does not readily reveal what needs to be "noticed at the level of understanding", or in Tzelgov's (1997) terms, the process can still run without being monitored. Real-time learning in this experiment is measured by the time taken to indicate whether a particular noun is abstract or concrete and the effect of the preceding novel verb on this response time. Participants are only required to focus on the noun in order to perform this decision. In that sense, the task may still be classified as automatic. 
Timeline:

Grammatical:

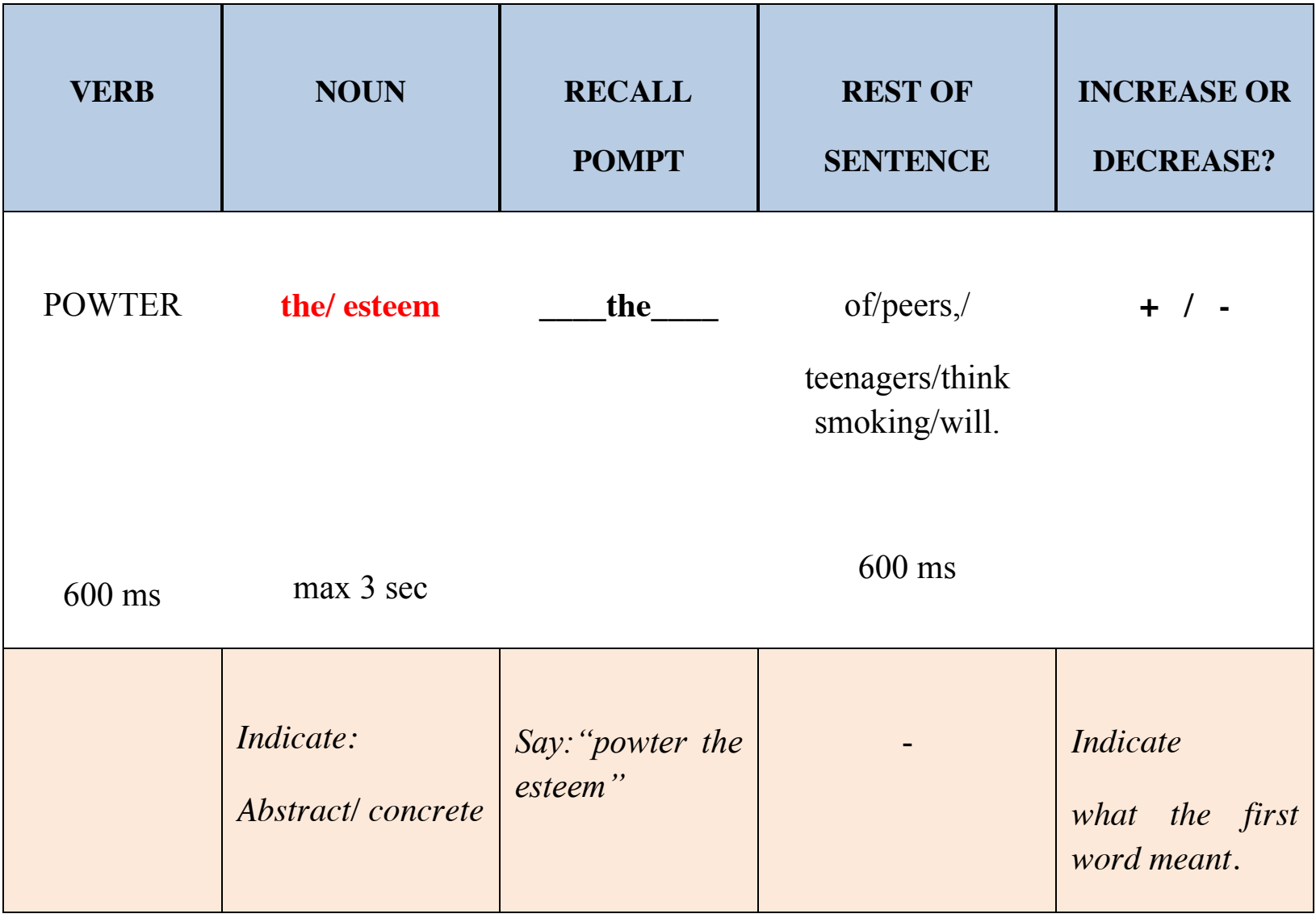

Ungrammatical:

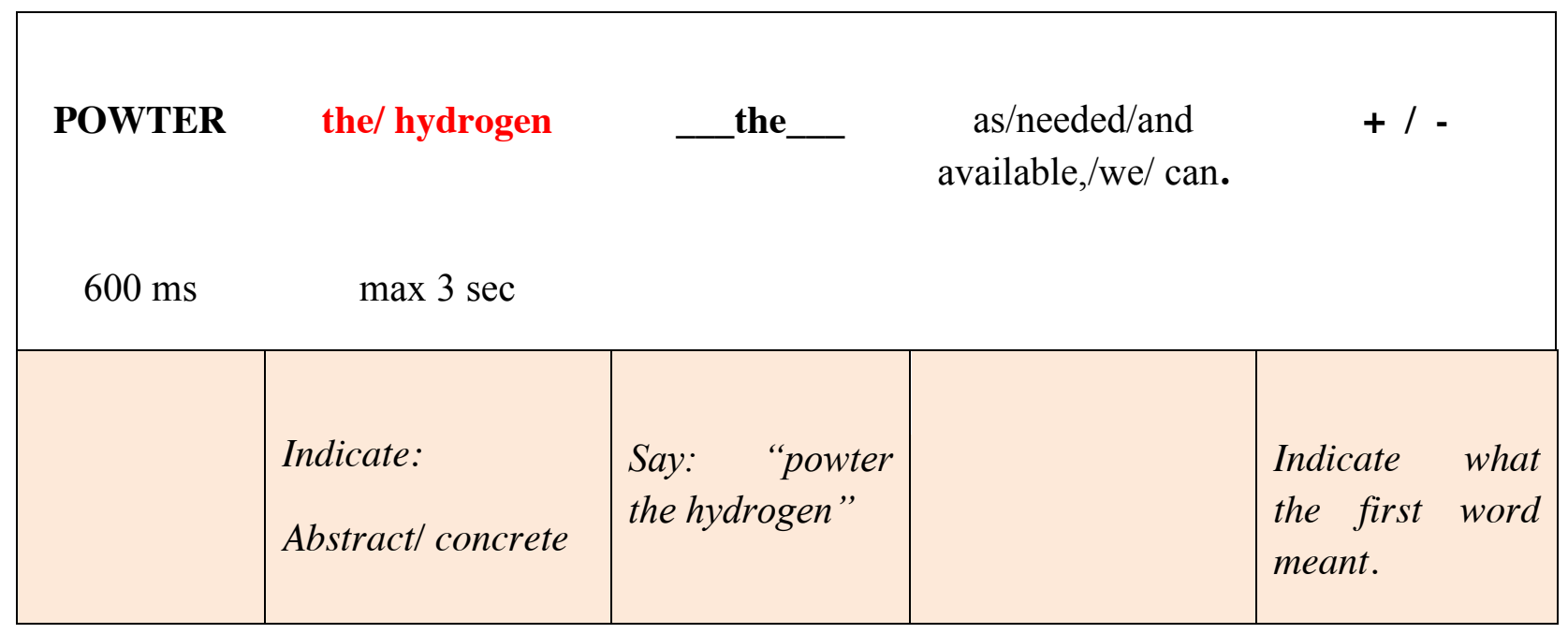

Figure 4.3. Experimental procedure with example sentences. 
Participants were divided into two groups, which involved rotations of grammatical and ungrammatical item between two versions.

To summarise the predictions: if semantic implicit learning of semantic preference patterns of novel verbs takes place automatically in real time during the course of processing the sentence, a slow-down of reaction times would be observed on the decision whether a noun is abstract or concrete when the pattern is violated.

A post-experiment questionnaire was carried out at the end, which was identical to that in Experiment 2a and other experiments, and which served as a means to elicit potential explicit knowledge.

\subsubsection{Results}

Out of 40 participants, 13 revealed at least fragmentary explicit knowledge in the postexperiment debriefing, and were classified as the 'aware' group. The remaining 27 participants were classified as the 'unaware' group. Two of this latter group were excluded from the analysis due to outlying performance (see below).

\section{Unaware participants}

Data from entire trials on which an error was made on the first decision (abstract/concrete) were removed. This was because participants were likely to have been distracted on the subsequent increase/decrease decision by just having had to correct themselves. Correct response times that were more than 2.5 standard deviations above the mean for the NG and NU conditions combined were classed as outliers. They were replaced with the next highest value (Winsorised). Each participant's mean response time over the critical trials was then plotted against their learning effect (reaction time difference between ungrammatical and grammatical trials) on a scatter plot in order to identify potential outlying participants. The plot is presented in Figure 4.5. It becomes clear that two people (points marked in red) are considerably removed from the homogenous cluster of other participants. Their mean reaction times are also slower than the others' by more than 2 standard deviations. These two participants' data were removed from the analysis as it was assumed that they used a different strategy while performing the task. Notably, reaction times longer than $3000 \mathrm{~ms}$ are not likely to reflect automatic performance. 


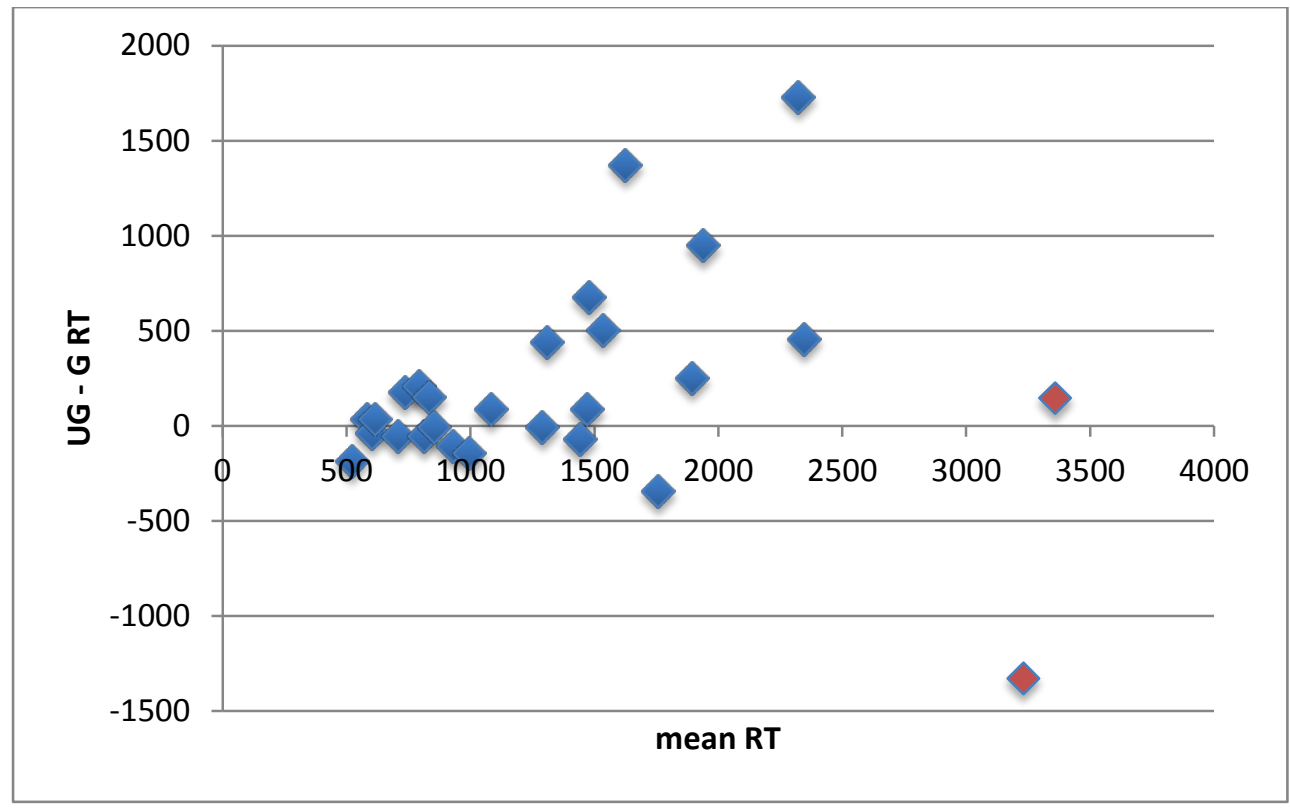

Figure 4.5. Relation between the mean reaction time and the learning effect (UG-G RT) among unaware participants, with outliers in red.

The results for the remaining participants are summarised in Table 4.1 below. An ANOVA was performed on the reaction times in two conditions ( $\mathrm{NG}$ and $\mathrm{NU}$ ), with the test version as a between-subjects factor. The 'unaware' group did not show any effects of grammaticality on the abstract/concrete decision on the noun, i.e. at the point of violation in the NU condition, $\mathrm{F}(1,20)<1$, thereby contradicting the prediction that the nonword would establish an expectancy for a noun of a certain semantic class. However, somewhat surprisingly, the same participants showed a significant learning effect on the increase/decrease decision that was made at the end of the sentences, $F(1,20)=5.11, p<0.05, \eta^{2}=0.20$. This finding suggests that semantic implicit learning exerts its influence on post-processing of the nonword-noun pair.

The mean error rate on the abstract/concrete decision for the NG items was $8.7 \%, S E$ $=0.25$, while for the NU items it was $7.7 \%, S E=0.23$. There was no significant difference between these rates. The mean error rate for the increase/decrease decision in the NG items was 25.2, $S E=0.23$, and the NU items $23.4, S E=0.26$ (note that these are the error rates over the trials that remain after exclusion of trials where an error was made on first decision). Again, there was no significant difference between the error rates in these two conditions. 
Such high error rates may have resulted from the fact that participants were not given any feedback on their answers, in order not to break the flow of the experiment. They also had no prior knowledge about which words meant 'increase' and which 'decrease'.

\section{Aware participants}

The 'aware' participants' data is also presented in Table 4.1. An ANOVA on this group's data did not reveal any significant differences in their reaction times between the two conditions, neither on the abstract/concrete decision $\mathrm{F}(1,9)<1$, nor on the second, increase/decrease decision $F(1,9)=1.70, p=0.23$. This lack of significance may possibly be due to the smaller size of this group than the 'unaware' group. Alternatively, taking time to work out the rule may have had a detrimental effect on performance.

The mean error rate on the abstract/concrete decision for the NG items was $6.7 \%, S E$ $=0.39$ and the NU items $7.8 \%, S E=0.37$, with no significant difference between these rates. The mean error rate for the increase/decrease decision for the NG items was 22.5, $S E=0.53$, and the NU items 23.9, $S E=0.56$. Again, there was no significant difference between the error rates in these two conditions.

\section{Native vs non-native speakers}

A post hoc analysis was carried out to check for potential differences in performance between the native English speaking participants and the non-native ones. An ANOVA revealed no interaction between grammaticality and native/non-native speaker status, $F<1.0$. For the native speakers the mean reaction times in the grammatical and ungrammatical conditions were $1394 \mathrm{~ms}(\mathrm{SE}=254)$ and $1704 \mathrm{~ms}(\mathrm{SE}=324)$ respectively, and for the non-natives 1283

$\mathrm{ms}(\mathrm{SE}=231)$ and $1558 \mathrm{~ms}(\mathrm{SE}=303)$ respectively.


Table 4.1. Results from Experiment 7. Mean reaction time (RT) in ms and standard error of the mean (SE)

\begin{tabular}{|c|c|c|c|c|c|c|c|c|c|}
\hline & & & \multicolumn{3}{|c|}{ Grammatical } & \multicolumn{3}{|c|}{ Ungrammatical } & \multirow[b]{2}{*}{ Effect size, $\eta^{2}$} \\
\hline & \multicolumn{2}{|l|}{$\mathrm{n}$} & Mean RT & SE of mean & \% Error & Mean RT & SE of mean & $\%$ Error & \\
\hline \multirow[t]{2}{*}{ Unaware } & 25 & Abs/Con & 977 & 47 & 8.7 & 996 & 49 & 7.7 & 0.02 \\
\hline & & Inc/Dec & 1101 & 81 & 25.2 & 1332 & 151 & 23.4 & $0.20 *$ \\
\hline \multirow[t]{2}{*}{ Aware } & 13 & Abs/Con & 1097 & 56 & 6.7 & 1093 & 70 & 7.8 & 0.00 \\
\hline & & Inc/Dec & 1478 & 204 & 22.5 & 1611 & 255 & 23.9 & 0.16 \\
\hline
\end{tabular}

$* p<0.05$ 


\subsection{Discussion}

Experiment 7 provides evidence for semantic implicit learning, detected through its influence on people's automatic behaviour. The fact that learning effects are apparent on the decisions involving purely the indication of whether a particular verb meant to "increase" or "decrease" is particularly compelling, if unexpected, as that meaning remained constant for each verb throughout the procedure (which, however, participants did not know upfront). It is surprising that the effect did not occur immediately at the point of rule violation (the appearance of noun), but with a delay. Before drawing conclusions it is important to ensure that the effect was not influenced by other words in the sentences or did not arise due to other factors than simply the relationship between verbs and noun-types.

Before proceeding to a more in-depth discussion of this finding another concern needs to be addressed. The effect in the experiment might potentially have been confounded by some other, unpredictable interaction between the non-words and other words in the sentences. It is therefore informative and useful to examine whether the same effect could be replicated without the sentence contexts, but simply in a situation where participants respond to phrases, such as gouble the significance or mouten the vitamins, giving the same two decisions as in the present experiment. The following study reports such an investigation and documents gradual methodological improvement towards ensuring that we are measuring true automaticity, where this is defined as an unmonitored process in which people's behaviour is unintentionally influenced, in real time, by factors unrelated to the task at hand.

\subsection{Pilot 3 - development of the "double decision priming" procedure}

This experiment was carried out as a preparation for an EEG study (reported in chapter 5) and aimed at establishing an optimal methodology for testing semantic implicit learning, which would also be compatible with EEG. For this reason, the procedure changed incrementally in stages.

Participants: 44 students of the University of Cambridge participated in the experiment. 25 of them were native speakers of English. The remaining group's mother tongues included: 
Afrikaans (1), Bulgarian (1), Cantonese (2), Czech (1), Danish (2), Dutch (1), German (3), Hindi (1), Macedonian (1), Malaysian (2), Polish (2), Russian (2).

\subsubsection{Materials}

The initial twelve participants were given version 1 of the experiment, which comprised the same verb-noun phrases which were used in Experiment 7 above, but stripped of sentential contexts. However, due to the exploratory nature of the studies presented here, it was deemed beneficial to test another set of nouns as well. The initial stimuli contained nouns which were highly similar to each other, often almost synonymous. Therefore it was of significant interest also to test a more heterogeneous noun-set (version 2). This offered an insight into the scope of the generalisation processes taking place in semantic implicit learning. The same motivation underpinned experiment 4 in Chapter 3. The stimuli used in version 2 are presented in Appendix F.

In both versions, there were two test lists. Everyone was tested on both lists, one immediately after the other with order counterbalanced. The nouns used to form ungrammatical items in list 1 were used in grammatical ones in list 2 and vice versa.

\subsubsection{Procedure}

Version $1 a$

The procedure was very similar to Experiment 7 with the difference that instead of being shown complete sentence stimuli, participants responded to phrases appearing word by word. In Experiment 7 sentence contexts helped participants determine whether a given non-word related to increasing or decreasing. This time, since the phrases were stripped of all surrounding words, participants were told in the instructions which novel verb indicated increasing and which decreasing.

The sequence of tasks was: 1) observe the novel verb on the screen, 2) on the subsequent appearance of the noun indicate as quickly and as accurately as possible whether it is abstract or concrete, no later than in 3 seconds, 3 ) on the "- / +" prompt indicate whether 
the initial novel verb was the one meaning to "increase" or to "decrease. Figure 4.6 below summarises the procedure.

Timeline:

\section{Grammatical:}

\begin{tabular}{|c|c|c|}
\hline VERB & NOUN & $\begin{array}{c}\text { INCREASE OR } \\
\text { DECREASE? }\end{array}$ \\
\hline POWTER & \multicolumn{2}{|l|}{ the/ esteem } \\
\hline $600 \mathrm{~ms}$ & \multicolumn{2}{|l|}{$\max 3 \mathrm{sec}$} \\
\hline & $\begin{array}{l}\text { Indicate: } \\
\text { Abstract/ concrete }\end{array}$ & $\begin{array}{l}\text { Indicate } \\
\text { what the first } \\
\text { word meant. }\end{array}$ \\
\hline
\end{tabular}

Figure 4.6. Procedure in Pilot 3: "Double Decision Priming".

\section{Version $1 b$}

After the initial 11 participants, an element of recall was added to the procedure to encourage deeper processing and possibly strengthen the effect. Every two stimuli, participants received prompts which required them to say into a microphone one of the phrases they have just seen. The prompt revealed either the first part of the phrase, for example "MOUTEN the or the second part: " the prestige", and participants were asked to pronounce the complete phrase. 
Version 2

Version 2 contained a new set of nouns. The motivation for this was to test whether the observed online effect relies on abstraction to rule or, rather, largely depends on the similarity of the test items to the training items. Because the second word set comprised much more heterogenous words than version 1, the similarity account predicted the effect might get weaker (just as it did in Experiment 4 compared to Experiment 3). In this version, all participants received recall prompts every 2 items. For the stimulus material see: Appendix F.

\section{Awareness measure}

Since the procedure was highly automatised, awareness was only determined in a postexperimental debriefing. Unlike in Experiment 7, participants were not shown any example sentences, but simply asked whether they noticed any pattern in the stimuli. The experimenter probed for any related knowledge, finally revealing the system and asking whether they became aware of it at any point. In cases where participants were not sure whether they did or not, the crucial question was whether they noticed that in the second half of the experiment phrases appeared which violated the pattern. If they did not notice this then they were classified as "unaware", as that indicates that, whatever knowledge they constructed during the task, they did not use it strategically.

\subsubsection{Results}

\section{Version $1 a$}

Among the 11 participants who performed version 1a, none reported awareness of the regularity in the final debriefing. The overall ANOVA with native/non-native as a betweensubjects factor did not reveal significant effects, neither on the first nor second decision. On the latter, $F(1,9)=2.30, p=0.16, \eta^{2}=0.20$. The mean learning effect (difference between $\mathrm{NU}$ and NG) was $47 \mathrm{~ms}$. At this stage it was hypothesised that adding recall prompts every two stimuli might increase this effect, and therefore provide better conditions for the development and testing of implicit learning. 
Version $1 b$

The following 6 people performed the experiment using the same word set with added recall every two stimuli. One of them became aware of the regularity, and was excluded from the current analysis. The group was too small to carry out a meaningful analysis. An initial check revealed that the introduction of recall slowed down the overall mean reaction times but at the same time considerably improved the learning effect. The mean difference between conditions among these 5 people was $94 \mathrm{~ms}$.

\section{Version $1 a \& b$}

A combined analysis of versions $1 \mathrm{a}$ and $\mathrm{b}$ revealed a close to significant learning effect on the second, increase/decrease decision with larger effect size than version $1 \mathrm{a}: \mathrm{F}(1,13)=4.64, \mathrm{p}=$ $0.051, \eta^{2}=0.26$.

Version 2

24 participants took part in this version, 15 of whom were native English speakers. 7 reported at least fragmentary awareness of the regularity and were classified as aware. Among the remaining 17 unaware participants, the learning effect on the second decision did not reach significance, with $\mathrm{F}(1,15)=1.50, \mathrm{p}=0.24, \eta^{2}=0.09$, and there was no interaction between grammaticality and the native/non-native factor. The effect size, albeit small, is comparable with Experiment 3 in Chapter 3, which also tested for broad generalisations. The learning effect in that experiment was significant, but the sample was also much larger, as it comprised 70 people, all of whom were English native speakers. It is possible therefore that this test version would also lead to significant results given a larger sample of people. This notion is corroborated with the overall analysis of the two versions.

\section{Overall analysis}

An overall analysis on the 33 unaware participants revealed a significant effect on the second decision $\mathrm{F}(1,30)=6.79, \mathrm{p}<0.05, \eta^{2}=0.19$. As in Experiment 5, there was no difference between conditions on the first decision: $F(1,30)<1$. The overall results are summarised in Table 4.2 below. 
Post-hoc native vs. non-native analysis

Although the overall analysis revealed no interactions between grammaticality and native/non-native speaker status on either decision, a post hoc analysis conducted separately for the two groups revealed that the effect on the increase/decrease decision was only significant among the native speakers: natives, $F(1,20)=5.32, p=0.03, \eta^{2}=0.21$, nonnatives, $F(1,10)=2.32, p=0.16, \eta^{2}=0.19$. It is difficult to draw conclusions at this stage, because the groups were of different sizes (21 vs. 11). An indication for the future is to keep the numbers balanced as nativeness may be a meaningful factor in semantic implicit learning of verb usage in context.

Table 4.2. Mean reaction times, ms (standard error in parenthesis)

\begin{tabular}{ccccccccc}
\hline & & & \multicolumn{2}{c}{ Grammatical } & & \multicolumn{2}{c}{ Ungrammatical } & \\
\cline { 3 - 4 } & & & Mean RT & SE of mean & & Mean RT & SE of mean & Effect size, $\eta^{2}$ \\
\cline { 2 - 6 } Overall & \multirow{2}{*}{32} & Abs/Con & 1068 & 57 & & 1049 & 57 & 0.02 \\
& & Inc/Dec & 558 & 47 & & 598 & 54 & $0.19^{*}$ \\
\hline
\end{tabular}

$* p<0.05$

Table 4.3. Mean reaction times (standard error in parenthesis) byspeaker type (native/non-native).

\begin{tabular}{|c|c|c|c|c|c|c|c|}
\hline & & & \multicolumn{2}{|c|}{ Grammatical } & \multicolumn{2}{|c|}{ Ungrammatical } & \multirow[b]{2}{*}{$\begin{array}{c}\text { Effect size } \\
\eta^{2}\end{array}$} \\
\hline & & & Mean RT & SE of mean & Mean RT & SE of mean & \\
\hline \multirow[t]{2}{*}{ Native } & 21 & Abs/Con & 1103 & 70 & 1087 & 72 & 0.02 \\
\hline & & Inc/Dec & 545 & 54 & 586 & 63 & $0.21 *$ \\
\hline \multirow{2}{*}{$\begin{array}{l}\text { Non- } \\
\text { native }\end{array}$} & 11 & Abs/Con & 1032 & 85 & 1011 & 78 & 0.03 \\
\hline & & Inc/Dec & 571 & 80 & 610 & 89 & 0.19 \\
\hline
\end{tabular}




\subsubsection{Discussion}

Pilot 3 ("double decision priming") replicates the findings from Experiment 7 and provides further evidence for semantic implicit learning, detected through its influence on people's automatic behaviour. The fact that learning effects are apparent on the decisions involving purely the indication of whether a particular verb meant to "increase" or "decrease" is particularly compelling, as that meaning remained constant for each verb throughout the procedure.

One objection which may be raised about both Experiment 7 and pilot 3 is the fact that people were explicitly provided with the critical semantic dimension - namely that nouns are to be distinguished with regards to whether they are abstract or concrete. This could be understood as limiting the extent of semantic learning taking place during the current procedure to mere sensitivity to the co-occurrences of a form (novel verb) and the following explicit concept (abstract/concrete). As mentioned above, the defence of this methodology is that the learning depends on the relationship between the novel verb and the following noun type, which is not mentioned upfront, and of which, as the post-experiment debriefing suggests, most people remained unaware. The recognition of this relationship is not part of the task at hand, and with the stimuli presented briefly and with the time pressure for response it is not surprising that people do not easily become aware of the regularity.

Still, the issue of interest especially to language pedagogues (particularly the advocates of the communicative approaches to teaching) is whether this sensitivity to semantic context only develops when the critical distinction is provided upfront or whether it can also be unconsciously induced. The series of false memory experiments in Chapter 3 suggests that indeed involuntary and unconscious activation of the key semantic distinctions can occur and that positive evidence from sentential contexts is enough to trigger it. Could that finding be corroborated using an automaticity-based procedure? It is also of interest to measure whether non-native speakers perform similarly to native speakers on such a task. Native speakers are expected to activate their concepts automatically and the activation of the key semantic distinctions is likely to occur. Whether it also occurs in non-native speakers is of particular pedagogical interest. The following experiment addresses these questions.

In terms of the automatic influence of implicit semantic knowledge on online processing, a further objection can be raised, namely that the target knowledge is not of negligible importance to the task. Should participants spontaneously decipher which verbs 
collocated with which type of nouns, it would considerably improve their performance on the test. However, that would only help them make abstract/concrete decisions. It doesn't undermine the findings though, as the slow-down in reaction time occurred on the increase/decrease decision. The following experiment modifies this procedure so that the target knowledge is irrelevant to the both components of the task.

\subsection{Experiment 8}

Participants: 49 students of the University of Cambridge participated in the experiment. Although none reported full awareness of the regularity, 3 of them made potentially helpful observations and were excluded from the subsequent analysis, which will focus on the unaware group. A further 3 participants were excluded due to problems with the task. Of the remaining group of 43 participants, 22 were native speakers of English. The other native languages included: Cypriot Greek (1), Czech (1), Urdu (1), German (3), Gujarati (1), Italian (1), Mandarin (1), Mongolian (1), Polish (6), Punjabi (1), Russian (2), Spanish (1), Thai (1).

\subsubsection{Materials}

Materials were identical to the second half of the double decision pilot above, Pilot 3 version 2 (Appendix F).

\subsubsection{Procedure}

The experiment followed a highly similar procedure to the double decision Pilot 3 above with the only difference being the type of decision required on the noun. While in Pilot 3 the first response participants were asked give was to indicate whether the noun was 'abstract' or 'concrete', here they were asked about the connotation they felt the noun had, either 'positive', 'neutral', or 'negative'. Examples of positive words were given such as 
'chocolate', 'holidays', and of negative such as 'horror'. Participants were informed that the choices were subjective.

The training, again, contained 60 well-formed phrases, where two verbs were always followed by abstract nouns, and the other two by concrete nouns. The testing part contained 76 phrases, in which 32 were critical, while the remainder provided further exposure to positive evidence. Half of the critical phrases were 'new grammatical' (NG) and half were 'new ungrammatical' (NU), the latter being those in which the verb was paired with a noun of the wrong abstract/concrete type. Two versions were created with the collocates rotated around versions such that nouns that were grammatical on one version were ungrammatical on the other, and vice versa.

Again, participants did not know that there were two separate stages in the experiment, although they did have a short break in between. Responses were collected using Cedrus ${ }^{\odot}$ response-pad and the experiment was programmed in SuperLab Pro 4.0.

\subsubsection{Results}

Outlying response times were identified and treated as in previous experiments. The data from the 43 unaware participants was first screened for outlying participants, both among the native and the non-native English speakers. Scatter plots of the relation between the mean reaction time and the learning effect (the difference between RTs for grammatical and ungrammatical trials) in these two groups is presented in Figure 4.7 below. People whose performance clearly deviates from the majority are marked in red. These participants (two from the native group) were removed from the subsequent analysis. 

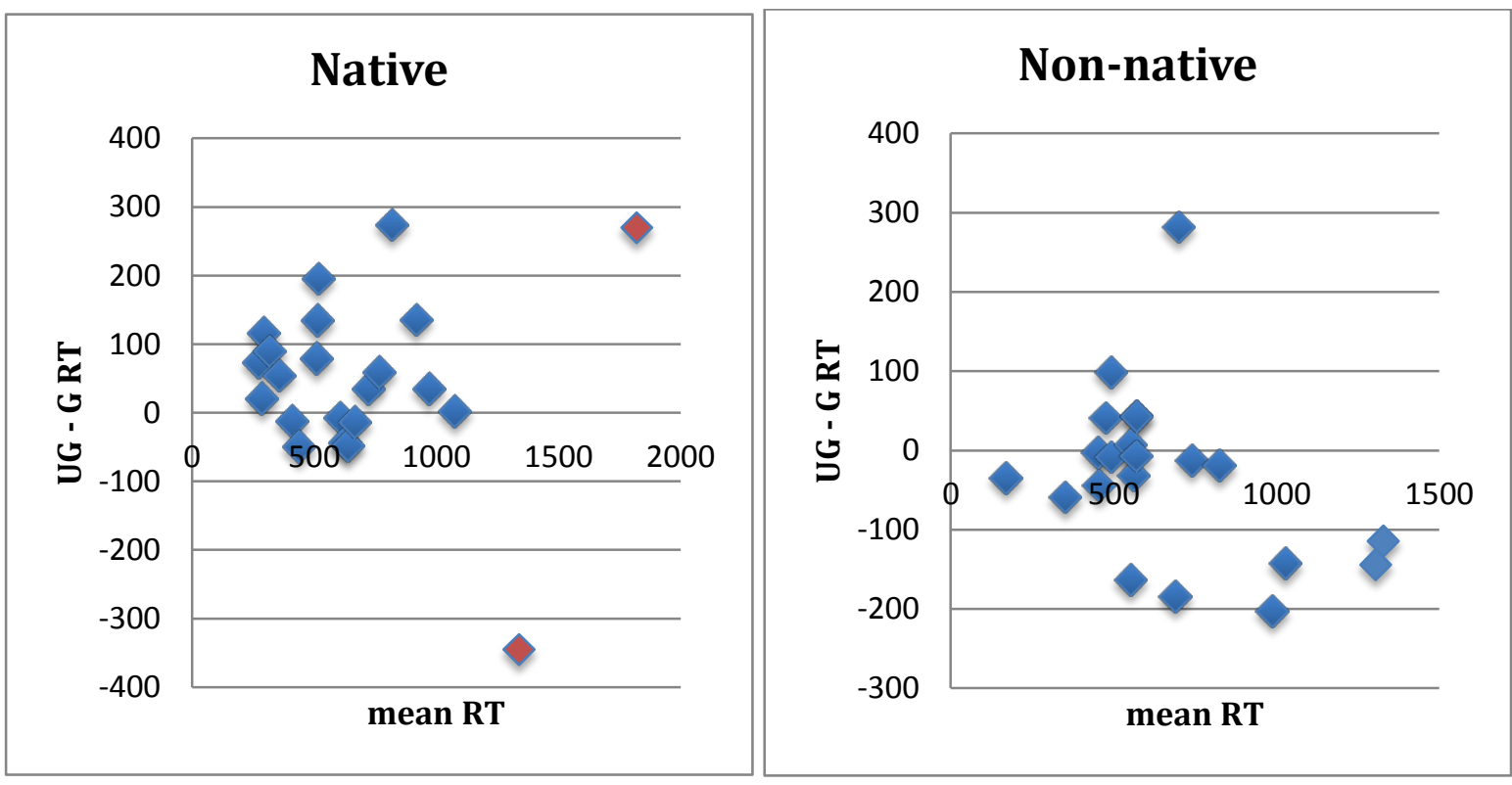

Figure 4.7. Relation between the mean reaction time and the learning effect (UG-G RT) among unaware participants, with outliers in red, for a) English native b) non-native English speakers.

The overall results are summarised in Table 4.4 below. An ANOVA was performed on the reaction times in two conditions (NG and NU), with list and 'native/non-native' as betweensubjects factors. Similarly to the previous studies, there was no effect on the first decision performed on the noun, $\mathrm{F}(1,37)<1$, and no interaction between grammaticality and the native/non-native group membership, $\mathrm{F}(1,37)<1$. An analysis of the second decision results revealed no overall effect $F(1,37)<1$, but a significant interaction between grammaticality and the native/non-native factor $\mathrm{F}(1,37)=37.0, \mathrm{p}<0.01, \eta^{2}=0.19$. The learning effect was only present among the native speaker group $\mathrm{F}(1,18)=9.13, \mathrm{p}<0.01, \eta^{2}=0.34$. The results of each group are presented in Table 4.5.

Errors were only counted for the second decision. The overall mean error rate in the NG condition was $6.1 \%$, while in the NU condition it was $6.7 \%$. There was no significant difference between the error rates in these two conditions. Error data for the native and nonnative group are presented in Table 4.5. There were no significant differences between conditions within the groups. 
Table 4.4. Mean reaction times (standard error in parenthesis).

\begin{tabular}{|c|c|c|c|c|c|c|c|c|c|}
\hline \multirow[b]{3}{*}{ Overall } & \multirow[b]{2}{*}{$\mathrm{n}$} & & \multicolumn{3}{|c|}{ Grammatical } & \multicolumn{3}{|c|}{ Ungrammatical } & \multirow[b]{2}{*}{ Effect size, $\eta^{2}$} \\
\hline & & & Mean RT & SE of mean & $\%$ Error & Mean RT & SE of mean & $\%$ Error & \\
\hline & 41 & Connotation & 1267 & 40 & - & 1267 & 38 & - & 0.00 \\
\hline & & Inc/Dec & 616 & 45 & 6.1 & 627 & 40 & 6.7 & 0.01 \\
\hline
\end{tabular}

Table 4.5. Mean reaction times (standard error in parenthesis) by native/non-native.

\begin{tabular}{|c|c|c|c|c|c|c|c|c|c|}
\hline & & & \multicolumn{3}{|c|}{ Grammatical } & \multicolumn{3}{|c|}{ Ungrammatical } & \multirow[b]{2}{*}{ Effect size, $\eta^{2}$} \\
\hline & \multicolumn{2}{|l|}{$\mathrm{n}$} & Mean RT & SE of mean & $\%$ Error & Mean RT & SE of mean & $\%$ Error & \\
\hline \multirow[t]{2}{*}{ Native } & 20 & Connotation & 1147 & 53 & - & 1140 & 54 & - & 0.005 \\
\hline & & Inc/Dec & 556 & 55 & 7.2 & 612 & 55 & 5.9 & $0.34 * *$ \\
\hline \multirow[t]{2}{*}{ Non-native } & 21 & Connotation & 1387 & 59 & - & 1393 & 53 & - & 0.002 \\
\hline & & Inc/Dec & 690 & 67 & 5.1 & 655 & 59 & 7.4 & 0.09 \\
\hline
\end{tabular}




\subsubsection{Discussion}

Experiment 8 provided the strongest evidence, among the studies reported in this chapter, for automatic influence exerted by implicit knowledge of semantic preferences on online processing. Since the participants' task was to indicate 1) whether the noun had a positive, neutral or negative connotation and 2) whether the verb indicated increasing or decreasing, whilst the learning was measured on reaction times to word pairs that were either matched or mismatched along the abstract/concrete distinction, the procedure can be said to tap automatic processing. Explicit knowledge of the relationship between verbs and abstract or concrete nouns is irrelevant to the task and would not facilitate participants' performance.

The results also revealed an interesting difference between native and non-native speakers' performance. The learning effect only reached significance among the native group. This suggests that only they could automatically activate the relevant features of the noun, which further led to implicit learning. For the non-native group, this activation may need to be stimulated explicitly, as in Pilot 3 (by making the relevant abstract/concrete decision), though this still needs to be confirmed. Pilot 3 involved too small a sample of nonnatives to determine this with confidence. Further research on the implicit online activation of semantic knowledge among the non-native speakers is of importance for language pedagogy.

\subsubsection{Overall discussion}

Overall, the experiments reported in this chapter point to the conclusion that implicit semantic knowledge does exert its influence automatically during online language processing. This finding confirms that the phenomenon is present in the natural use of language. The effect only emerged experimentally when participants were encouraged to process the stimuli deeply for meaning (Experiment 7, Pilot 3, Experiment 8), while the appearance of the visual forms and a recall task did not stimulate it. It seems therefore that in experimental conditions where there is time pressure on the response, the participants' attention and depth of processing have to be appropriately stimulated by the task. By comparison, in the experiments in Chapter 3, learning effects emerged after participants simply read sentences for meaning. It appears therefore that extra measures to encourage 
deeper processing are not necessary outside the lab, and a natural linguistic context is enough to stimulate implicit learning. Together, these observations point to the conclusion that the phenomenon of semantic implicit knowledge exerts its influence in the natural use of language both online and offline.

Across the experiments in which the automatic influence of implicit semantic knowledge was found, the effect consistently emerged at the second decision (increase/decrease), and not at the point where the violation took place (the appearance of a right/wrong noun type; first decision). This could be taken as a reflection of the fact that different semantic features are activated on different time-courses (Yee et al., 2011). Alternatively, the consistently delayed effect could indicate that semantic implicit knowledge exerts its influence only in post-processing, making the method of priming with two consecutive decisions a useful tool to detect it. Further replication studies with different semantic distinctions than the abstract/concrete one would be highly informative.

The native and non-native groups were only purposely contrasted in the final experiment, while in the previous ones the analysis of their potentially different performance was post hoc. Considering the marked difference in performance between them on Experiment 8, it would be of interest and importance in the field to determine whether this finding would be replicated using other semantic distinctions.

\subsection{Chapter summary}

Chapter 4 addressed the issue of whether semantic implicit knowledge exerts its influence in language processing online, in real time. In this way it complements the findings from Chapter 3 where it was shown that such knowledge can be shown to indirectly shape participants' active recall memory (as evidenced in their false memory patterns).

Chapter 4 began with a definition and a brief overview of literature on automaticity and its operationalisation both in SLA and psychology.

Pilots 1 and 2 tested automaticity in a priming paradigm, following the same training as in the Chapter 3 experiments. The test question for the target was: "did you see that word in the first 
part?" It was concluded that this methodology did not stimulate sufficient depth of processing of the prime words to produce an effect.

Experiment 7 provided participants with a smooth transition between training and test, with the same task throughout. Both training and test stimuli were in the form of full sentences, presented word by word in a scrambled order such that the verb was always at the beginning. Despite the fact that participants indicated their choices of the verb meaning only after the whole sentence was presented, that decision revealed a significant slow-down in reaction times on the ungrammatical trials. There was no difference on the first decision, whether the noun was abstract or concrete, even though that was made at the moment when the violation occurred.

Pilot 3 documents a gradual development of what can be called a "double decision priming" procedure, in which participants see phrases on the screen and make decisions regarding each word but in the reverse order. The aim was to devise a procedure which would be compatible with EEG (electroencephalography), and therefore sequential presentation was required. The challenge consisted of the fact that, as mentioned in the discussion following the two first pilot studies, sequential presentation is often detrimental to implicit learning. The results from the "double decision priming" replicated the pattern from Experiment 7, showing a delayed effect on the second, increase/decrease decision.

Experiment 8 provides the most solid evidence for the automatic influence of semantic implicit knowledge. Only in this experiment was the task which participants performed irrelevant and not beneficial for their learning target. Interestingly, automaticity was only revealed among the native speakers and not the non-natives. It is suggested that native speakers activate their concepts fully automatically, which allows them to show learning effects even in speeded conditions, which non-native speakers do not do. Further research on this phenomenon could be very useful from the pedagogical point of view. 


\section{Chapter 5}

\section{Real-time ERP response to semantic}

\section{implicit learning}

*The empirical material presented in this chapter is a result of the collaboration with Dr Nitin Williams, University of Reading (now at the University of Cambridge), who designed the new EMDbased method for ERP signal analysis applied in this study. The ERP data was analysed by Dr Nitin Williams, and is also included in his PhD thesis (N. Williams, 2011). The figures were reproduced with permission, and the Results section (marked with *) presents the findings included in Dr Nitin Williams' PhD thesis as well.

\subsection{Introduction}

ERP is a particularly good technique for investigating brain responses in language processing as well as in implicit learning research due to its high temporal resolution. Different components have already been identified as being related to different stages of sentence processing. Early syntactic parsing is assumed to be reflected in the LAN (Left-Anterior Negativity), which is prominent around the anterior electrodes. The LAN is followed by an N400 which, in a sentence processing context, reflects the cognitive cost of online integration of semantic information. An example sentence which would yield a large N400 on the final word, reflecting a high integration cost, is She drank her tea with a microphone. Then, a P600, also known as the Syntactic Positive Shift (SPS), most prominent over the posterior electrodes, is usually believed to reflect syntactic reanalysis or revision and is elicited by such syntactic phenomena as ungrammatical sentences (e.g. She drank her tea and sing), garden 
path sentences, complex sentences with a large number of thematic roles, or long distance dependencies. Thus, the identification of specific ERP components for different aspects of sentence processing imposes a neuro-biological constraint for models of language processing, which will need to account for the observed sequence of ERP effects (Brown \& Hagoort, 1993).

The method has already established itself in SLA research and has led to insights into the cognitive mechanisms of language processing which would not be possible otherwise. For example, the study by Morgan-Short et al. (2007) compared the ERP responses to syntactic processing among learners of an artificial language Brocanto2, trained under explicit and implicit conditions ${ }^{15}$. It was found that although the two groups performed similarly at both low and high proficiency, their ERP responses to syntactic violations differed. The group that underwent implicit training showed an N400 at low proficiency, and showed anterior negativity followed by P600 followed by late anterior negativity at high proficiency. The latter pattern is similar to native-like syntactic processing. The presence of the N400 suggests that at the early stages of learning, at least in this condition, people treat clauses as meaningful units, most likely relying on rote memory. On the other hand, the group in the explicit training condition showed no significant effects at low proficiency, and anterior positivity followed by a P600 at high proficiency, which is not reminiscent of native-like processing. Therefore, in all, only "implicit training" led to an electrophysiological signature characteristic of native speakers. The authors concluded that uninstructed, "implicit training" conditions yield superior results to the explicit ones in terms of native-like attainment, which in turn confirms the popular view that language learning through immersion is better than in a classroom setting.

Batterink \& Neville (2011) reported an interesting study of incidental word learning, in which participants were given both "implicit"16 and explicit tests. ERPs were recorded as participants read stories with pseudowords appearing at multiple points, embedded either in consistent meaningful contexts $(\mathrm{M}+)$ or inconsistent, meaningless ones $(\mathrm{M}-)$. They were subsequently tested on two tasks, targeting different types of responses: lexical decision ("implicit test"), and recall and recognition ("explicit test"). It turned out that M- words

\footnotetext{
${ }^{15}$ Implicit conditions in the study by Morgan-Short et al. (2010) are similar to the "incidental" conditions defined here in that they involve "non-instructed" behaviour. However, they do not presuppose lack of awareness of the rules; learners' awareness was not measured in the study.

16 "Implicit" as used by Batternk \& Neville (2011) is akin to how "automatic" is used in this dissertation; i.e. uncontrolled. However, no claims about awareness were made, and awareness was not measured.
} 
elicited a larger N400 than M+ words, indicating that the M+ words were easier to integrate. Interestingly, participants' performance on the lexical decision task showed neither a significant behavioural nor ERP difference. It is suggested that the explicit representations of word meanings can develop rapidly, whereas implicit representations may require more extensive exposure or more time to emerge.

A natural question arising here is: is it even possible to observe ERP responses to implicitly learned regularities at all? Note that the studies by Morgan-Short et al. (2007) and Batterink \& Neville (2011) are not indicative of this as they do not measure unconscious knowledge, but rather different modes (syntactic, semantic) of automatic processing. There is evidence in the literature for N400 effects in subliminal semantic priming (Kiefer, 2002). Also, P600 has been reported in an implicit SRT task (Christiansen, Conway, \& Onnis, 2012). There is also evidence of an N1 component in AG learning that is independent of the behavioural response (Schankin, et al., 2011). Overall there is surprisingly little research on implicit learning using ERP, and apparently nothing on semantic implicit learning. The study reported in this chapter addresses this lacuna, using a novel method for analysing the ERP signal, based on Empirical Mode Decomposition, allowing comparisons between single trials.

\subsection{Empirical Mode Decomposition (EMD)}

The problem with applying traditional ERP signal analysis methods to research on such phenomena as learning is the fact that it involves averaging over multiple repetitions of the same stimuli based on the assumption that the ERP signal is constant from trial to trial. This assumption does not always hold, and in fact between-trial variations in the signal could be linked to aspects of cognition. The standard voltage averaging method, therefore, might cancel out potentially informative variations in the data, which could be particularly informative for gradual processes such as learning, believed to induce mental changes over time. There are currently only a few methods for analysing the ERP signal based on individual trials, but none of the methods are completely data-driven and unsupervised while allowing for the ERP to be non-stationary between trials. A method for analysing the signal based on Empirical Mode Decomposition, which has these characteristics, was developed by Dr Nitin Williams, (ref thesis), and applied in a variant of the "double decision priming" paradigm developed in Chapter 4. Empirical Mode Decomposition (EMD) is a data-driven 
method which extracts oscillatory modes intrinsic to the data by identifying time scales characteristic of individual oscillatory modes. It decomposes a signal into a small number of Intrinsic Mode Functions (IMFs) which represent the oscillatory modes contained in the data. The counterpart of the IMF in Fourier analysis is the simple harmonic component. However, the IMF is much more general than the harmonic component as it can be modulated both in terms of amplitude and frequency, while the harmonic component is constant in both amplitude and frequency. The amplitude and frequency modulations are possible because the decomposition depends on the local characteristic time scale of the data. (Williams, 2011).

Applying an EMD-based ERP method to an implicit learning study has a particular benefit over the behavioural methods used to date. Most demonstrations of implicit learning (including the ones reported here), within such frameworks as AGL, contextual cueing, false memory paradigm, etc., usually tap into knowledge retrieval, and, are based on the assumption that if knowledge is influencing behaviour without awareness, then it has most likely also been acquired without awareness (Shanks, 2005; cf. Tzelgov, 1997 and Introduction to Chapter 4, for the distinction between unawareness and processing without conscious monitoring). This opens the procedure up to criticism that such demonstrations cannot be claimed in fact to address the implicit learning process, until that fundamental assumption is verified (cf. Carrón \& Bly, 2007). A single-trial based analysis of an ERP signal, such as the EMD-based method, would allow us to see changes over time while still applying relevant measures of awareness and assuring automaticity in the learning procedure, by tapping into the learning process itself. Below is a study reporting a replication of Variant 2 in Pilot 3 of Chapter 4, with the additional application of ERP measurement, and the application of the EMD-based method of signal analysis. Gradual changes in the signal, with the absence of awareness until the end of the experiment, would demonstrate that implicit learning is indeed a gradual process and constitute a first attempt to measure the process neurologically in real time.

It is also of interest to see what components emerge for the violation items. If semantic implicit learning is indeed a semantically-based phenomenon, as has been assumed in this dissertation, then it is expected that an N400 would occur. On the other hand, the violations in, e.g., powter the honey when powter normally occurs with abstract nouns, is not exactly the same kind of violation as that which occurs in She drank her tea with a microphone. While the latter introduces a challenge to a coherent interpretation in the light of general world knowledge, the former violates an agreement with regard to a particular 
semantic feature which is critical, and forms a rule. In that case, one might expect a P600, which is what Morgan-Short et al. (2010) reported for article-noun agreement violations, but only in highly proficient participants. The N400 was present at the less proficient level. Considering that participants in the current study encountered the novel target verbs for the first time (so could be considered to be of low proficiency) this would suggest a higher likelihood of an N400. ${ }^{17}$

\subsection{Experiment 9}

Participants: 18 native speakers of English, 16 right-handed and 2 left-handed, all above 18 years of age (between 19 and 46), with a mean of 27; all had no history of neurological or psychological disorders. 13 were male, 5 female.

Equipment: Data was collected using a 64-channel BrainVision EEG system from Brain Products $\mathrm{GmbH}$ in the electromagnetically shielded EEG lab at the Centre for Integrative Neuroscience and Neurodynamics (CINN), University of Reading, UK. Data was sampled at $1000 \mathrm{~Hz}$ and, for each participant, all electrode impedances were below $10 \mathrm{k}$.

\subsubsection{Materials}

Materials were the same as those in Version 2 of Pilot 3, reported in Chapter 4. The stimulus material is presented in Appendix F. As before, four target novel verbs were introduced: POWTER and MOUTEN meaning 'increase', GOUBLE and CONELL meaning 'decrease'.

\subsubsection{Procedure}

\footnotetext{
${ }^{17}$ It has been braught to the author's attention that the N200/N1 could be predicted as well, which will be addressed in the future analysis.
} 
The procedure was the same as Version 2 of Pilot 3, reported in Chapter 4. Each participant received each list in succession, with the order of lists counterbalanced. However, the order of trials within each list was the same for all participants.

Specifically, the experiment contained 3 blocks of phrases, each containing 78 phrases. In the first, training block MOUTEN and CONELL co-occurred only with concrete nouns while POWTER and GOUBLE co-occurred only with abstract nouns. In blocks 2 and 3 , there are 16 violations each of the collocational rule, each preceeding or following a critical grammatical item, both of which forming a pair - henceforth referred to as 'stage'. EPrime 2.0 was used for stimulus presentation. The phrases were visually presented on the screen, word-by-word, verb followed by a noun. He verb appeared for $600 \mathrm{~ms}$. On the immediately following noun, participants participants were instructed to press the '.' button if it was a concrete noun and ' $z$ ' if the noun was abstract. Participants had up to 3 seconds indicate their choice. After making this abstract/concrete decision, the screen changed and participants were presented with a question about the verb in the phrase. If the initial verb meant 'increase' they were asked to choose "+" on the screen, by pressing the '? Key, or 'decrease' bychoosing “_." and pressing the ' $z$ ' key. The next phrase was presented only after the participant answered this verb question. Every two trials participants were also prompted to recall aloud the last two phrases. This was done to make the participant to engage with the task. Further, participants were asked not to move or blink while answering questions about the nouns and verbs in the phrases.

\subsubsection{Results}

\section{Behavioural results}

A paired-sample t-test revealed no significant difference overall on either the first (abstract/concrete) decision on the noun, $\mathrm{t}(17)=0.62, \mathrm{p}=0.54,($ Mean $\mathrm{G}=1250,79, \mathrm{SD}=$ 350.96 , Mean $\mathrm{UG}=1265,47, \mathrm{SD}=311,29$ ) or on the second (increase/decrease) decision, $\mathrm{t}(17)=1.680, \mathrm{p}=0.11$ (Mean $\mathrm{G}=580,81, \mathrm{SD}=249.49$, Mean $\mathrm{UG}=60.98, \mathrm{SD}=258.73)$. After dividing each list into halves, the effect was significant on the second half of each list, $\mathrm{t}(17)=2.24, \mathrm{p}=0.04$ (Mean $\mathrm{G}=548.40, \mathrm{SD}=231.28$, Mean $\mathrm{UG}=581.47, \mathrm{SD}=263.64) . \mathrm{In}$ other words, it emerges over the $2^{\text {nd }}$ and $4^{\text {th }}$ quarters. Due to the design of the experiment, the same items always appeared in either the first or second half of a list. It seems therefore that 
the effect may be restricted to a subset of the items (which is understandable in light of the role similarity plays in implicit learning, see section 3.9 above). The effect occurs on the subset of items that appear in the second half, which are rotated around the G and UG conditions between the lists, but kept in the same position, so it is still a valid comparison. Overall, there is some evidence of an implicit learning effect.

*ERP results

\section{First decision}

\section{Conventional Grand average comparison}

On the first decision a right-lateralised low-frequency effect was found in the fronto-temporal region (F8, FT8, FC6). Subject 13 was not included in the analysis because of excessive contamination of the data with eye-blink artifacts. As shown in Figure 5.1, the response to the New Grammatical (normal) condition (-0.43) was more positive than the response to the New Ungrammatical (violation) condition (-1.66). An analysis of p-values revealed that the effect was strongest between $200 \mathrm{~ms}$ and $400 \mathrm{~ms}$. A paired-samples t-test showed that the mean amplitude for this time-window was different between conditions $(\mathrm{p}<0.05)$. FC6 was the electrode at which the effect was most prominent. The threshold frequency (ft) for EMD denoising was set at $7 \mathrm{~Hz}$.

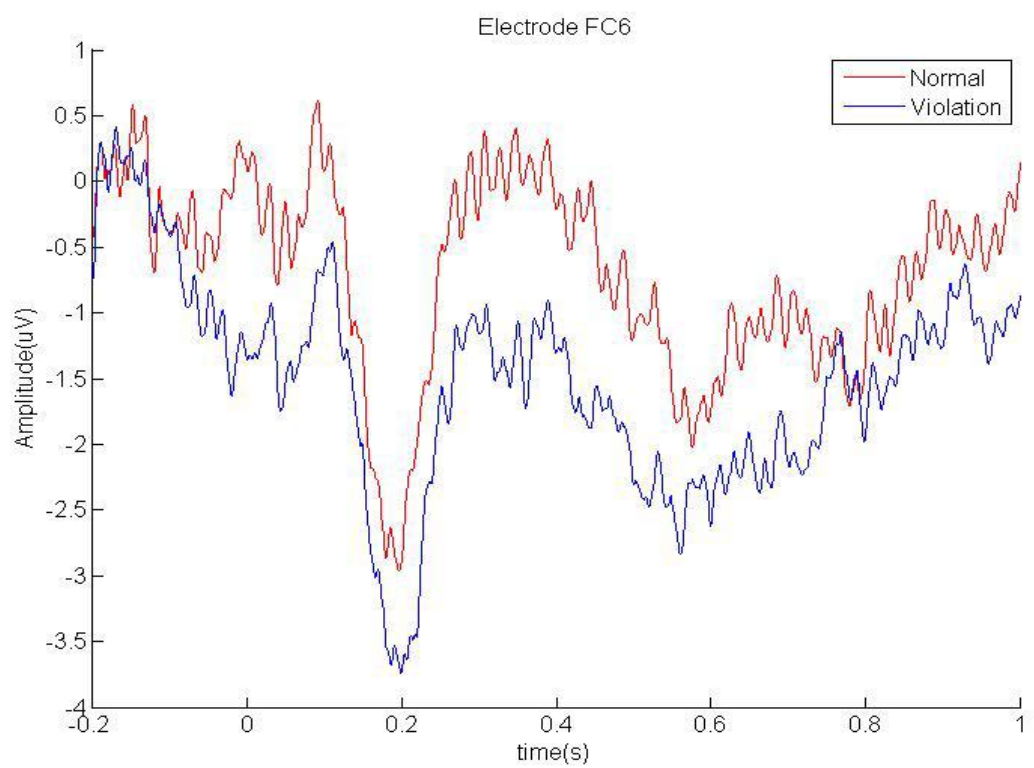

*Figure 5.1. First decision, Grand average comparison - "normal" and "violation" condition (electrode FC6) 
This finding might suggest that the ERP signal proved more sensitive than the reaction time method, and revealed an effect already on the first decision. Such a conclusion can only be drawn with caution, because according to the signal depiction in Figure 5.1, the lines for the two conditions diverge already at point 0 , although not significantly. The result therefore remains inconclusive.

Another possible interpretation of the discrepancy at point 0 is that participants already have an expectation as to whether the noun will be grammatical or ungrammatical before it actually appears. Actually, there was a regularity in the stimuli in that respect. The test phase followed an order such that every sixth item was ungrammatical (i.e., exposure, exposure, grammatical, exposure, exposure, ungrammatical, exposure, exposure, grammatical, exposure, exposure, ungrammatical, where "exposure" refers to grammatical filler trials). However, since the sequence does not predict the actual abstract/concrete response (just the grammaticality of the verb-noun combination) it is hard to see how learning of the sequence could be reflected in the ERP signal.

\section{Empirical Mode Decomposition}

EMD denoising (clearing the signal from artifacts) at $7 \mathrm{~Hz}$ was done for all trials from both conditions (based on the signal-to-noise criterion) ${ }^{18}$, on electrode FC6. Single-trial analysis was conducted to verify whether the learning could be tracked by effect size between responses to the two conditions (Grammatical and Ungrammatical) across 32 stages (pairings of Grammatical and Ungrammatical trials, which appeared one after the other). The slope of the regression line turned out to be near-significantly non-zero $(\mathrm{p}=0.06)$. Figure 5.2 shows the effect size values plotted for each stage, along with the linear regression line. Figure 5.3 shows an ERP image of the change in effect size across 32 stages, for each time point. The gradual decrease in effect size across stages is reflected by the gradual change (from top to bottom of the figure) in colour: from yellow and orange to green, blue and dark blue. Note that this change is visible between $200 \mathrm{~ms}$ and $400 \mathrm{~ms}$.

\footnotetext{
${ }^{18}$ Calculated by subtracting each post-stimulus value by mean of activity from $0 \mathrm{~ms}$ to around $200 \mathrm{~ms}$ prestimulus; carried out to control for potential effects resulting from pre-stimulus processing.
} 


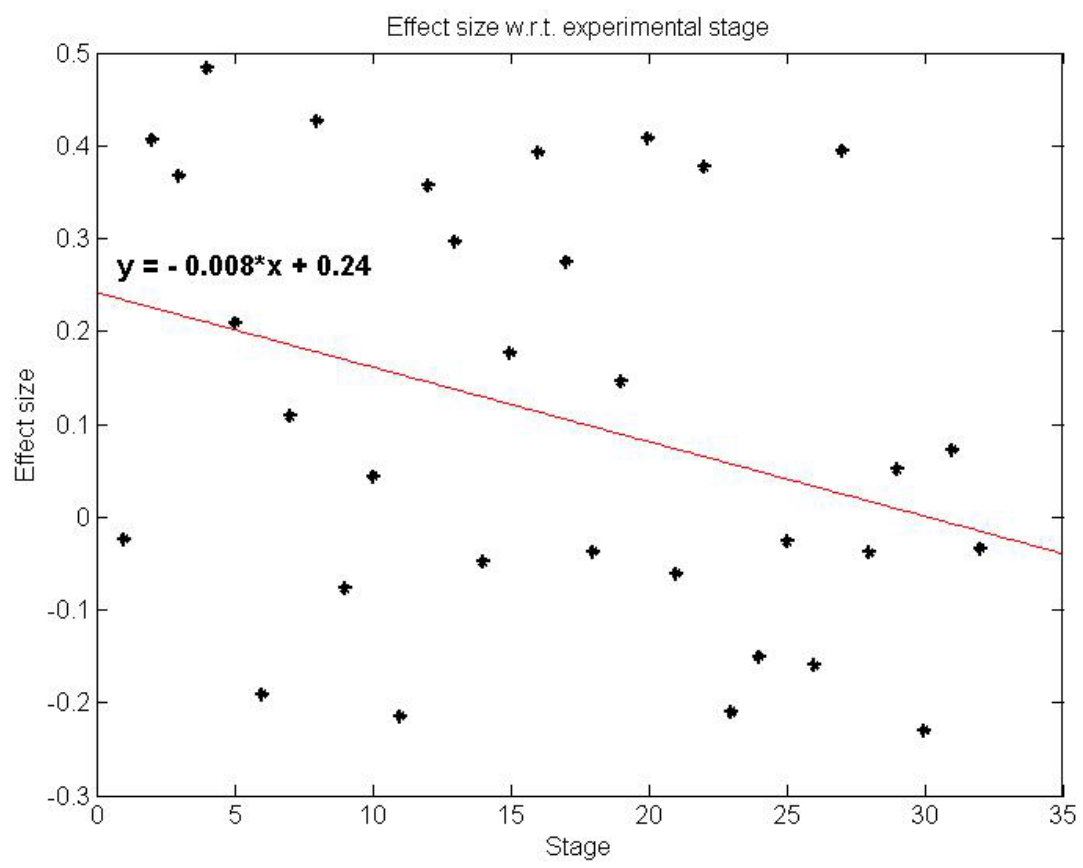

* Figure 5.2. First decision, effect size with respect to experimental stage (electrode FC6)

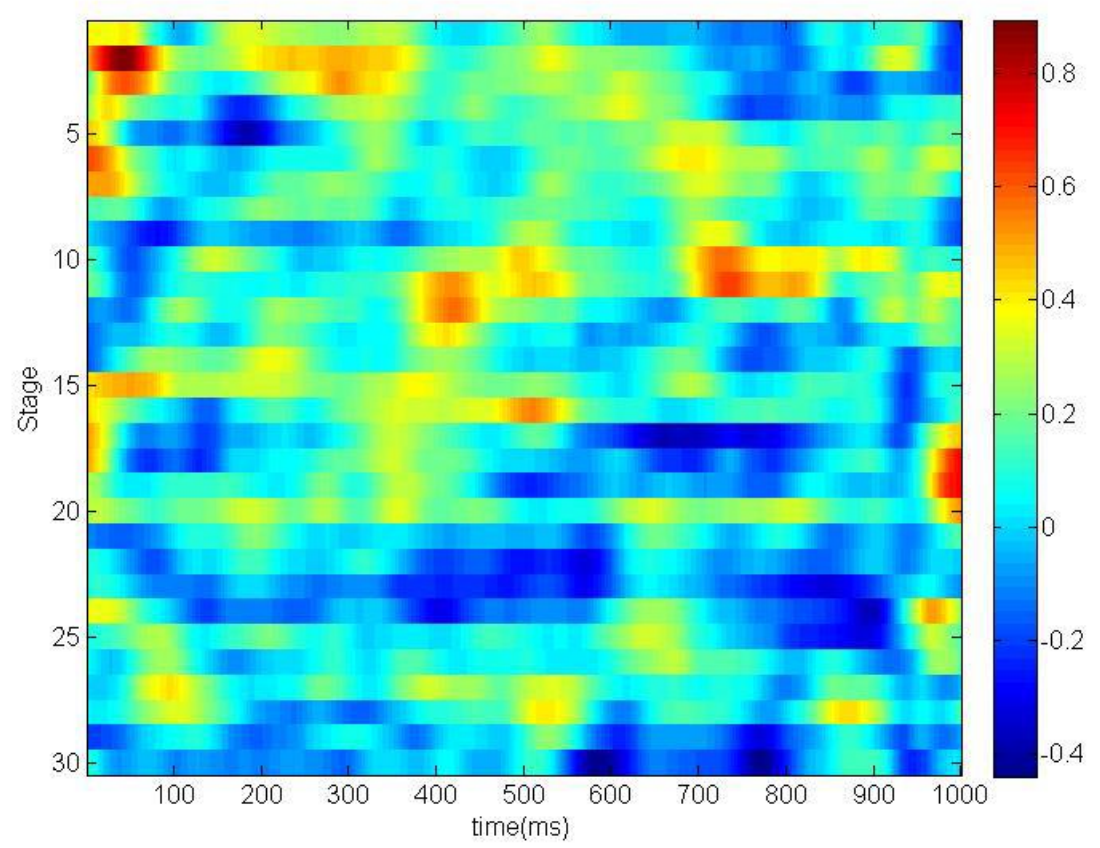

*Figure 5.3. ERP image of effect size for each sample for each Experimental Stage. Image was smoothed over Experimental Stages with window width of 3 stages. 


\section{Second decision}

\section{Conventional Grand average comparison}

Two effects emerged in response to the second decision. The first was a low-frequency effect in the pre-frontal and frontal regions (Fp1, Fpz, Fp2, AF4, AF8). Here participants 5, 13 and 15 were not included in the analysis because of excessive contamination of the data with eyeblink artifacts. As shown in Figure 5.4, the response to the New Ungrammatical (violation) condition (-4.03) was more positive than response to the New Grammatical (normal) condition (-1.31). Based on inspection of p-values, the effect was found to be particularly strong between $100 \mathrm{~ms}$ and $500 \mathrm{~ms}$. A paired-samples t-test confirmed that the mean amplitude for this time-window was different between conditions $(\mathrm{p}<0.05)$. Fpz was found to be the electrode at which the effect was most prominent.

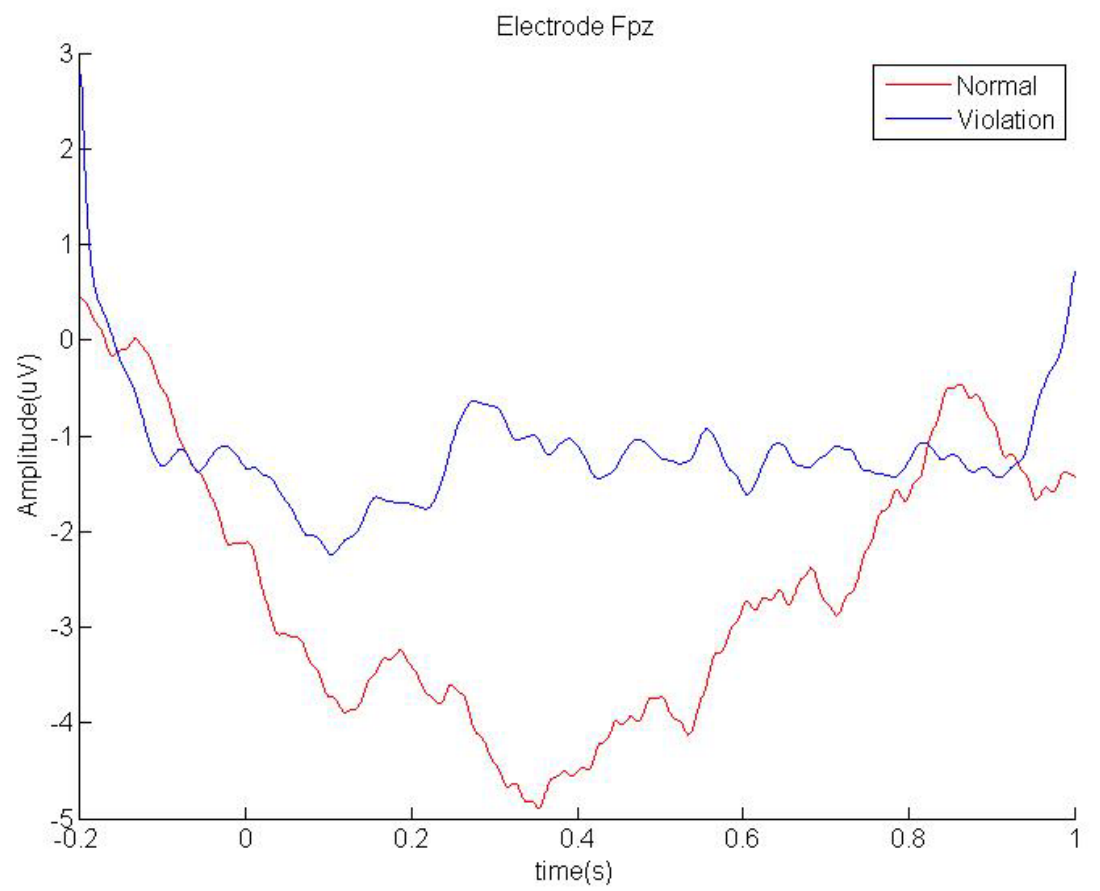

*Figure 5.4. Second decision, 1st effect, Grand average comparison - "normal" and "violation" condition (electrode Fpz). 
The other effect in response to the second decision was left-lateralised and localised in the central region $(\mathrm{Cz}, \mathrm{C} 1, \mathrm{C} 3, \mathrm{FC} 1, \mathrm{CPz})$. For this analysis, data from all 18 subjects was included. As shown in Figure 5.5, the response to the New Grammatical (normal) condition (0.73) was more positive than response to the New Ungrammatical (violation) condition $(-0.10)$. Based on the analysis of p-values, the effect was found to be particularly strong between $200 \mathrm{~ms}$ and $500 \mathrm{~ms}$ (most likely an N400). A paired-samples t-test revealed that the mean amplitude for this time-window was different between conditions $(\mathrm{p}<0.01)$. C3 turned out to be the electrode at which the effect was most prominent.

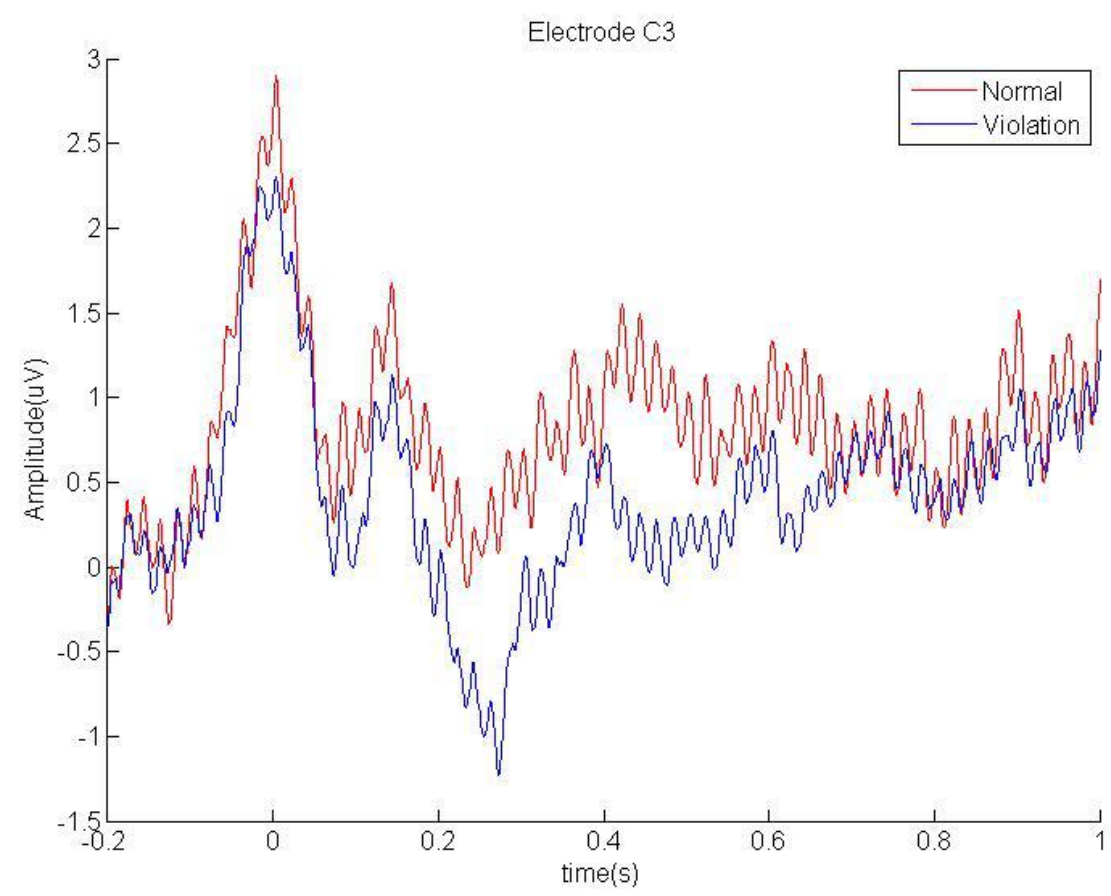

*Figure 5.6. Second decision, 2nd effect, Grand average comparison - "normal" and "violation" condition (electrode C3)

\section{Empirical Mode Decomposition}

Threshold frequency (ft) for EMD denoising was set at $1 \mathrm{~Hz}$ (based on the signal-to-noise criterion). On the first effect, single-trial analysis revealed that the variation in effect size between responses to the two conditions, across 32 stages, did not yield a linear regression line of significantly non-zero slope $(\mathrm{p}>0.05)$. This is illustrated in Figure 5.6 below. Nor was the regression slope significantly non-zero on the second effect (Figure 5.7). 


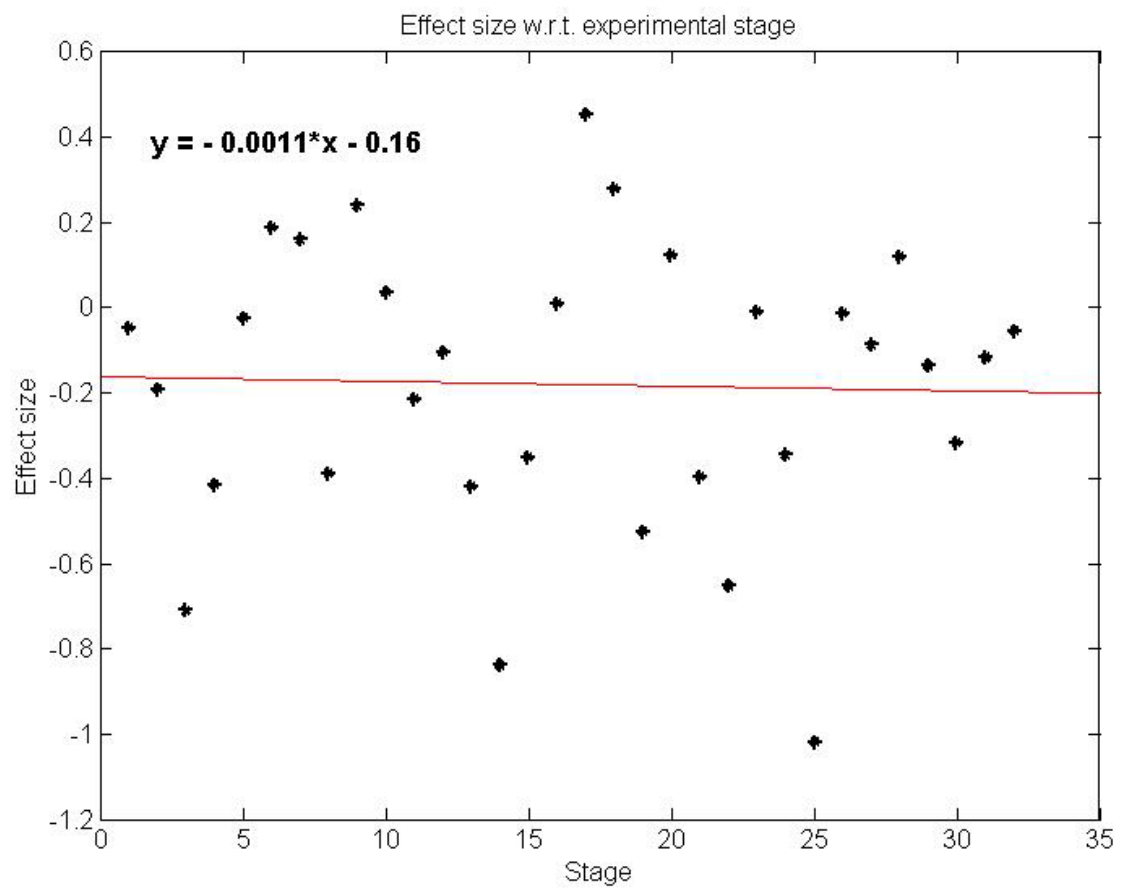

*Figure 5.5. Second decision, $1^{\text {st }}$ effect, effect size with respect to experimental stage (electrode Fpz).

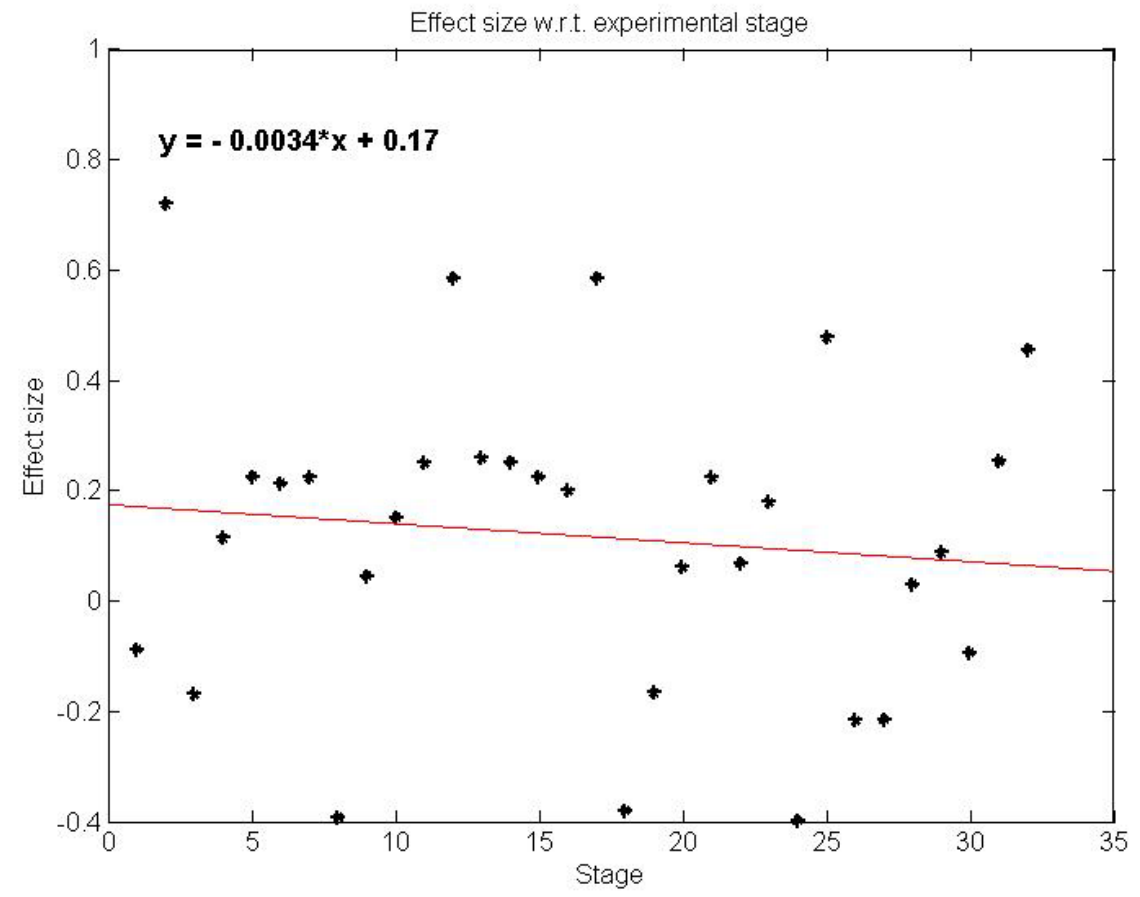

*Figure 5.7. Second decision, $2^{\text {nd }}$ effect, effect size with respect to experimental stage (electrode C3). 


\subsubsection{Discussion}

This study reported an investigation into the ERP brain response among people performing the "double decision priming" task targeting semantic implicit learning developed in Chapter 4. There was some evidence for the expected N400 responses on both decisions. This was least convincing on the first (abstract/concrete) decision due to the (non-significant) deviation in the waveforms prior to stimulus onset (see Figure 5.1), and its strongly right-lateralised prefrontal localisation. However, more convincing evidence was obtained for the second (increase/decrease) decision, where the effect was predominantly left-lateralised in the centro-parietal region and its latency was consistent with an N400 (see Figure 5.6). This latter result supports the assumption that the effects obtained with this paradigm are indeed semantically-based, and as such do not behave like syntactic or morphosyntactic violations, which typically elicit a P600.

There was some evidence that the ERP signal was sensitive to changes over time, but this was only obtained for the effect on the first decision, which unfortunately was the hardest to interpret. There was a marginally significant reduction in effect size (which reflects increasing amplitude in the ungrammatical condition by comparison with the grammatical condition). This may reflect an increasing sensitivity to a regularity in the trial sequence, although it is hard to see how this could affect the brain response in this case. There was no evidence for a similar decrease in effect size on the second (increase/decrease) decision, even though it was here that the traditional grand average analysis provided the most convincing evidence of a learning effect.

The results suggest that an ERP method may be more sensitive than a behavioural measurement, given that no overall behavioural effects were obtained in this study. There was, however, some evidence for an effect in reaction time on a subset of the items. The weakness of the behavioural effect is most likely caused by the noun set being relatively heterogeneous. It is therefore striking that differences between conditions were obtained in the ERP data.

Finally, it must be remembered that these ERP effects were obtained with participants who claimed to have no awareness of the selectional preference rule. They therefore represent the first evidence for unconscious ERP effects in the domain of semantic implicit learning. 


\subsection{Chapter summary}

The study presented in this chapter documents the first attempt to track implicit learning as it happens in real time. It began with an overview of currently identified ERP responses to specific types of linguistic processing (e.g., N400 for semantic integration). The overview was followed with a presentation of the innovative Empirical Mode Decomposition-based method for analysing an ERP signal allowing comparisons between individual trials. The study reported was done in collaboration with Dr Nitin Williams. Conventional grand average analyses revealed a significant left-lateralised N400 effect on the increase/decrease decision in the absence of awareness of the regularity, and in the absence of a behavioural effect. This suggests that the ERP method might be highly sensitive to implicit learning effects. The EMD analysis provided some evidence for a change in the learning effect over the course of the experiments, suggesting that this method has promise for the exploration of brain responses in implicit learning experiments. 


\section{Chapter 6}

\section{Overall discussion}

\subsection{Summary of findings}

The research documented in this dissertation addressed the issue of whether people implicitly form semantic generalisations over sets of nouns collocating with novel verbs (semantic preferences) and therefore become able to distinguish legitimate and non-legitimate use of those verbs in context. Results obtained through a series of 9 experiments ${ }^{19}$ using four different experimental methods provided support for the claim. The methods were based on automaticity and subjective measures of awareness and included a pen-and-paper study, false-memory experiments, "double decision priming" for detecting delayed priming effects, and a novel ERP-based technique. In order:

Chapter 2 provided the first empirical support that learners of English as a foreign language develop implicit lexical knowledge pertaining to the use of four, previously unknown words: diminish, deplete, mitigate and truncate. Learners were tested in the classroom, and the implicitness of learning was determined by questions about confidence and source judgements at each test question.

Chapter 3 provided further evidence for learning semantic generalisations, this time using a more controlled method based on semantic preference patterns arranged around a $2 \times 2$

\footnotetext{
${ }^{19} 14$ experiments including pilot studies and different variants of Experiments 2 and 6.
} 
factorial design. Learning was determined by the size of a false-memory effect and a postexperiment questionnaire. Participants who reported no knowledge of the semantic preference patterns at the end still showed a preference for the new verb-noun pairings which followed the correct pattern over those which did not. Applying a false-memory procedure to a familiarity task has benefits over the popularly used grammaticality judgements, as it prevents participants from constructing ad hoc hypotheses about test items. In the falsememory procedure, all the participants have to indicate is whether they remember the stimulus or not. Furthermore, the experiments reported in this chapter presented a novel, indirect measure of (functional) awareness, involving the analysis of correlations between the learning effect and confidence. Experiment $6 \mathrm{~b}$ in particular provided strong evidence for implicit learning. The method was verified in an explicit variant of the task (Experiment 5), in which participants who were not only aware but also able to use their knowledge strategically (as revealed by the post-test) were clearly distinguishable from those who did not perform perfectly well on the post-test. This confirmed that the method is sensitive to strategic knowledge use.

Chapter 4 examined whether semantic implicit knowledge exerts its influence automatically in real time language processing. While the effect in chapter 3 can already be considered as automatic, as learning the target regularity was not part of the task requirement (participants could still perform the task without knowing that there was a regularity between verbs and noun-type pairings at all), it still required participants to intentionally introspect their memory while doing it. Although unlikely, it is possible that they could still have had knowledge which they did not reveal in a verbal report or confidence measure. So, this series of five experiments presents the gradual development of the "double decision priming" method, and the application of a more common measure of automatic performance, namely reaction time. The procedure led to the detection of a learning effect, thereby providing further evidence for the implicit learning of semantic preference patterns. Interestingly, throughout this series of experiments, the effect consistently appeared on the second decision, suggesting that at least in this case, the effect arises after processing, exhibiting a delay from the moment the word is presented.

Chapter 5 provided early evidence that the development of semantic implicit learning may be traceable overtime using a novel single-trial analysis of an electroencephalographic (EEG) signal based on an empirical mode decomposition (EMD) denoising method developed by Dr 
Nitin Williams. Furthermore, the N400 component was detected at an earlier stage than the behavioural responses did, suggesting high sensitivity of ERP for measuring implicit knowledge. The presence of the N400 component adds more evidence to the claim that semantic implicit learning is indeed meaning-based, rather than form-based, or rule-based.

While implicit learning has usually been shown to occur at the perceptual form-level, for example in artificial grammars, or visual sequences, the research reported here has demonstrated that abstract, semantic level regularities are learnable implicitly as well.

\subsection{What do people learn?}

Traditional research on implicit learning has dismissed the idea that people may learn abstract rules governing the stimuli they encounter, and instead stresses the role of the consciously perceived similarity between the materials on which people are trained and tested (Shanks, 2005; Perruchet, 2008). The mainstream consensus seems to be that the implicit learning mechanism operates on chunks of which people might be aware. Therefore often an item with chunks which are consciously perceived as familiar is judged as grammatical, regardless of its actual grammaticality (as discussed in Chapter 1).

The story of implicit learning emerging from this dissertation, however, is more complex. To use an analogy to artificial grammar learning, participants may perceive test items as being chunked in a certain way, which leads them to accept the items, but in fact this can also arise when participants have never encountered those chunks and their impression is actually a consequence of the abstract structure of the grammar or a given system (cf. Knowlton \& Squire, 1996 - dissociating chunk strength and grammaticality). In the experiments reported here (Chapter 3) participants accept new word combinations claiming they had seen them, when in fact they had not.

It becomes apparent though that although people's knowledge in the current context is independent from perceptual forms, the similarity between training and test items still remains the key factor determining the learning effects. This time it is not perceptual, but rather semantic similarity. The account for the effects obtained could therefore be analogous to that coming from the form-level implicit learning research (e.g., Vokey \& Brooks, 1992) In the semantic implicit learning literature, Newell \& Bright (2002) observed that participants in their study encoded time in a rough manner and choose test items closer to the ones from 
training, regardless of whether they followed the 6-12 rule or not. The role of semantic similarity also emerged in the experiments reported here.

In Experiment 1 no learning was detected when new words were used in the "extended" contexts, which were grammatical but dissimilar from training. Also, in the false memory experiments in Chapter 3, Experiment 4 (generalisations), despite the large sample (61 unaware), revealed a relatively small effect size by comparison with the equivalent Experiment 3. This illustrates that the knowledge participants develop is not in the form of a rule: powter/gouble + ABSTRACT, conell/mouten + CONCRETE. Also, in Experiments 2 and 3 the participants' generalised notion of the noun collocates must consist of a more constrained set of possibilities than in Experiment 4. If their knowledge was in the form of a rule we should not observe a difference in effect sizes. In the reaction time experiments in Chapter 4 as well, the effect size diminished when the word set was changed to a more heterogeneous one (Pilot 3 versions $1 \mathrm{a}, 1 \mathrm{~b}$ combined $\eta^{2}=0.26$ versus version $2 \eta^{2} 0.09$ ).

This is reminiscent of other research on implicit leaning in language. Rebuschat \& Williams (2011) found evidence for learning word order regularities, but only in the form of templates based on the training items, rather than word placement rules. It was found that the group who had received exposure endorsed significantly more grammatical structures than the controls ( $71 \%$ versus $36 \%)$, but did not differ on the ungrammatical structures $(47 \%$ and $51 \%$ ). Thus, as much as there is evidence for learning of the syntactic patterns underlying the training sentences, the fact that the experimental group did not reliably reject ungrammatical items suggests their knowledge was not based on a rule, "assuming that rules enable a clear identification of what is ruled out, as well as what is ruled in" (Rebuschat \& Williams, p. 242). Similarly, Williams \& Kuribara (2008) found that participants implicitly learned statistical properties of a semi-artificial language based on Japanese to which they were exposed, but did not acquire the general rule of scrambling. In all, there is ample evidence that higher-level implicit learning, including semantic learning, is similarity-driven in much the same way as form-level implicit learning. The only difference is that the similarity itself is to be measured at a higher (e.g. semantic) level.

Interestingly though, it seems less likely that results are dependent upon similarity to a particular item, than similarity to the overall set. Distinguishing between these two explanations is important in the light of the debate between association and feature-based models of semantics. Although either account could explain the results obtained in Chapter 3, in the automaticity-based experiments in Chapter 4 participants still showed the semantic 
implicit learning effects despite the fact that they were unlikely to have had enough time to consciously recall a specific item. Therefore, possibly the only way to uphold an item similarity matching account is to claim that this occurs unconsciously.

\subsection{How do people learn?}

Learning taking place in the experiments presented here can be conceived as gradual abstraction over instances stored in memory. Consecutive activation of overlapping semantic features of words (here, nouns) and the context in which the given words appear (here, with which novel verb) results in the reinforcement of their relation without the learners' awareness (see section 3.10 above for further elaboration). It is plausible that such learning may be an instance of more general, cross-situational learning, which could even underlie the learning of content words' meanings (Smith \& Yu, 2008). Interestingly, it has been recently reported that infants at 6-9 months old already know the meanings of many common nouns (Bergelson \& Swingley, 2012). This is much earlier than was previously assumed. Predominantly, it has been claimed that meaning is learnt subsequently to speech segmentation, whereas (Bergelson \& Swingley, 2012) demonstrate that the two are likely to go hand in hand. Although the finding that infants know referential meaning of common nouns does not entail they learn them implicitly, it does demonstrate that learning vocabulary does not necessarily require elaborate explicit hypothesis testing in which second language learners are often believed to engage.

\subsection{Learning without noticing?}

As mentioned in Chapter 1, the seminal statement made by Schmidt $(1990,1995)$ is that "noticing", understood as attention and low level awareness, is the necessary and sufficient condition for learning to occur. Only the material which is noticed can be converted from input to intake.

Arguably, in Experiment 8 in Chapter 4, participants do not "notice" the crucial abstractness/concreteness distinction and are surprised to hear afterwards that this is what was tested. Of course the point of the automaticity-based procedure was to eliminate the potential 
effects of conscious knowledge of this regularity should it have arisen, but it is highly unlikely that it did, based on participants' reports. The learning effect reported in that experiment, as well as most experiments reported here, does emerge among the "unaware" participants, who denied having "noticed" the regularity in the final debriefing.

What does and what does not necessarily need to be noticed, then, for learning to take place? Based on the findings from Experiment 8, it is not necessary to be aware of the crucial aspect of the input items, but rather process the input in sufficient depth for it to be activated. The effect was not significant when participants were not forced to process the words for meaning (Pilot 1 and Pilot 2 here, Leung \& Williams 2012), but reached significance even when the meaning which was processed (positive/neutral/negative) was irrelevant to the learning target (abstract/concrete). However, Schmidt (1995) also proposed that stimuli outside focal attention and therefore outside awareness might only activate preexisting memory representations, and only do so subliminally. Perhaps such a level of activation occurred among the native speakers, and not non-native speakers in Experiment 8. This casts doubt that non-native speakers' performance on semantic implicit learning tasks of this kind could ever be similar to that of the native speakers, as the former do not have the same preexisting concepts to activate as the latter do.

\subsection{Comparison of methods}

Assuming that all the presented methods legitimately address implicit knowledge, comparing them brings the conclusion that the more artificial the test is (the more different from the natural language use), the more processing reinforcement is needed. The false memory experiments in Chapter 3 do not involve response time pressure, and the effect of semantic implicit knowledge emerges without the need even to suggest the importance of nouns following the target verbs. On the other hand, with timed presentation and response pressure, the learning procedure seems to require additional procedures to assure deep enough processing. 


\subsection{Implications for models of cognition}

In the light of the debate concerning whether implicit learning exists at all, the material collected in this dissertation points to the conclusion that it does. While Lovibond \& Shanks (2002) and others argued that even simple Pavlovian conditioning relies on conscious predictive relationships between events, it has to be said that their counterarguments are based on the research addressing form-level phenomena. Perhaps the lack of generalisation or abstraction beyond the given input in those experiments reflects the fact that the material is not linked to any higher-level representation, and therefore not naturally prone to be generalised from. In semantic implicit learning, participants acquire knowledge about relationships involving conceptual representations, which are naturally abstract. Perhaps therefore it is more promising to investigate the potential of implicit generalisations on naturally generalisable material, such as language, music, mathematics or motor learning.

\subsection{Indications for future research}

\section{Model of semantic knowledge}

As argued in Chapter 3 (section 3.8.5) this research could significantly contribute to one of the major debates in linguistics, between feature-based and association-based theories of semantic knowledge. The first and important step should be to dissociate the role of association strength and semantic similarity (based on people's ratings) in semantic implicit learning. Targeting unconscious rather than conscious knowledge should eliminate the effects of participants obscuring their natural reflexes by conscious strategies.

\section{Role of similarity}

A number of experiments reported here suggested that similarity matching is an important factor influencing implicit learning. The automaticity-based experiments in Chapter 4 suggest that this may not be similarity to a specific item, but rather similarity to the overall set. Furthermore, Chapter 5 suggests that this effect may be gradually increasing over time, again suggesting that it is not driven by similarity to a specific item (or at least not 
conscious similarity matching). Still, this conclusion is not definitive, and in the light of the fact that most of the false-memory literature would suggest that particular item similarity is key, further research on the matter would be highly beneficial for our understanding of the mechanisms underlying semantic implicit learning and the apparent emerging generalisations.

\section{Learning content words}

As mentioned above, the exact mechanism of implicit learning processes, including those underpinning the implicit learning of language, is yet to be fully understood. One of the issues of interest is whether the referential meaning of words can be learnt this way. The material presented in this dissertation addresses learning of semantic collocational behaviour, which can be argued only to involve 'fine-tuning' of representations. It has been argued that such knowledge belongs to the lexicon as opposed to vocabulary, and therefore its learning may proceed implicitly (Paradis, 2004). Referential word meaning, on the other hand, requires integration of information across different cognitive systems, which may crucially depend on intact declarative memory (Ellis, 1994, 2005), which in turn is believed to rely on the hippocampus. However, most empirically based arguments for such a position come from the studies reporting impaired vocabulary learning in amnesia (Gabrieli, Cohen and Corkin, 1998). Yet the fact that those patients have problems learning vocabulary does not necessarily entail that the process is always explicit. Rather, it may be that just an intact hippocampus is essential. Therefore, it might still be possible to demonstrate implicit learning of referential word meanings in the healthy population.

\section{Role of $L 1$}

Research on implicit learning in language is still a young discipline, and only relatively recently has evidence been reported that this kind of learning may interact with prior linguistic knowledge. In Experiment 8 of this dissertation, only native speakers of English showed learning, while non-natives did not. Considering that the same learning target regularity (novel verb with either abstract or concrete noun) was learnt by both native and non-native speakers in the false-memory experiments in Chapter 3, this finding suggests that the native language may provide rapid automatic activation of semantic information, which in non-native speakers may require some reinforcement, at least under speeded-response conditions. 
One way in which the first language could shape implicit learning is by providing a prior knowledge base. In light of other studies reported by Leung \& Williams $(2011,2012)$ it would be most useful to investigate the role which the first language plays in subsequent implicit learning (both for language and in other domains). Investigating the role prior knowledge plays in facilitating or constraining implicit learning could be illuminating in the light of one of the major debates in linguistics, namely that between generativism and emergentism. The methodology developed in this dissertation could be extended to investigate implicit learnability of various aspects of grammar, such as argument structure. Careful, typologically motivated predictions would need to be made to determine whether different L1s would have predictable impact on implicit learning. According to emergentist theories, any differences would find their explanation in domain-general cognition. White (2003) implies that from the UG perspective "acquisition of L2-like argument structure is not inevitable, depending on which language is L1 and which is the L2". Earlier she argues that "the failure to lose L1 argument structure arises in situations where the L1 grammar yields a superset of the possibilities permitted in the L2. That is, positive L2 input for restructuring the lexical entry is lacking" (White, 2003, p. 239). More empirical evidence for distinguishing the role of L1and the universal principles for second language learning is still needed.

\section{Role of the explicit "seed" in semantic implicit learning}

Apart from understanding what influence prior linguistic knowledge may have on second language learning, it is also interesting to investigate the role explicit knowledge may have or even have had in the experiments reported here. Is explicit knowledge a type of scaffolding, on which implicit learning can subsequently operate and fine-tune the existing representations? In other words, are the two systems, if separate, dependent upon each other? Or, on the other hand, is the fact that participants in the current research, as in many semantic implicit learning experiments to date, were provided with some explicit aspect of meaning, actually a crucial condition for implicit learning of this kind to take place? In the current experiments participants were at least given the increase/decrease dimension of the novel verbs' meaning; in Williams and Leung's (2012) studies they are told the new particles are like English determiners but indicate a near/far distinction; in Goschke (2007) experiment the effect emerges on a naming task, which also, at least in part, relies on explicit knowledge. 
The degree of dependence of implicit upon explicit learning could be addressed by adapting the methods presented in this dissertation. A possible way to test the issue would be to introduce variability in the explicit dimension of meaning of novel verbs. So for example, powter could mean 'to increase' in some training sentences, and 'to decrease' in other. Should similar learning effects be obtained to the ones reported here, it would seem that implicit learning does not require a fixed, explicit "seed" to subsequently fine-tune, but rather it is an independent process. In light of the findings from Goujon (2010) such a situation is not unlikely. There, semantic contextual cueing only emerged when learners had an opportunity to categorise the scene prior to the visual search, even if the scene they categorised was different from the one on which they performed their search. Whether these findings can be extended to language is an open question. Language could be different in that respect, as participants may have natural expectations that words have a constant meaning, and introducing inconsistencies could impair learning. Determining the role explicit knowledge plays in implicit learning clearly remains an issue waiting to be addressed, especially since it would be directly informative for language pedagogy.

\section{Consolidation and production}

The potential for further applications of the phenomenon of semantic implicit learning depend on how long-lasting the effects developed through training are. Initial promising. Comparison of different training techniques on consolidation and durability of effects would benefit the field.

Also, an inspection of the influence of semantic implicit learning tasks on free production would be useful in view of their potential pedagogical applicability, as well as their implications for the theory of second language acquisition. Production has been explored in an implicit learning context in an Elicited Oral Imitation test developed by Rod Ellis (2005). At the moment it is not clear how implicitly gained knowledge influences spontaneous language use,

\section{Neural plasticity}

The constant improvement and development of techniques for analysing brain activity has potential to provide new insights into the nature of learning, both implicit and explicit, at a level of detail previously unachievable. Chapter 5 of this dissertation showcases an initial 
application of a new ERP technique based on empirical mode decomposition (EMD), which by accessing the signal from individual trials offers a method for tracking gradual neural changes occurring over time. This, as well as other neuroimaging techniques, could address the issue of the impact of implicit learning on neural plasticity. Such information has the potential for developing further applications of this research in the area of cognitive therapy.

\section{Learning in impaired populations}

Addressing the question of the extent to which impaired populations are capable of implicit learning could prove very informative for neuroscience. For example, testing whether amnesic patients could develop semantic knowledge implicitly would be informative about the nature of hippocampal and cortical learning. The hippocampus is assumed to be responsible for storing specific memories which the cortex then uses to extract similarities and form generalisations. Impairment of the hippocampus is a common cause of memory loss and it is currently debated whether or not this damage prevents the development of semantic representations. Up to now, the studies which suggest no semantic learning in amnesia relied on procedures (e.g. word list learning) in which healthy individuals would use explicit strategies. It would be useful to re-address the issue using procedures which stimulate the development of implicit semantic knowledge, rather than explicit knowledge.

In addition, investigating semantic implicit learning among autistic people could shed light on which abilities are preserved and which are not in this disorder. There is evidence that implicit learning abilities are intact among the autistic population. This conclusion is based on their performance on such tasks as artificial grammar learning, serial reaction time, contextual cueing and probabilistic classification learning tasks (Brown Aczel, Jimenez, Kaufman, Grant, 2010). However, cognitive processing in autism has been characterised by a difficulty with the abstraction of information across multiple stimuli or situations (e.g. Minshew, Meyer, \& Goldstein, 2002; Ropar \& Peebles, 2007) and its subsequent generalisation to novel stimuli or situations (e.g. Ozonoff \& Miller, 1995; Swettenham, 1996). This makes individuals with autism seemingly unlikely to show semantic implicit learning effects. It has been assumed that prototype formation, a process of creating a mental summary representation of multiple experienced stimuli belonging to the same category, is also impaired in this group. However, in a recent study, Froehlich et al. (2012) challenge this assumption, arguing that although explicit categorisation is impaired, prototype formation is 
comparable to healthy controls. The tasks they used to demonstrate this difference arguably relied on explicit and implicit processing respectively. After exposure and classification of multiple dot displays, participants were asked to indicate whether they remembered seeing a given display or not (test for prototype formation), and if they responded in the affirmative, the second task was to indicate to which of the four categories the display belonged. It turned out that the participants with autism demonstrated intact prototype formation and did not differ significantly from the controls. One of the measures was false recognition of previously unseen prototypes. Participants with autism falsely recognized previously unseen prototypes to a reliably greater degree than they accurately recognized familiar training and repeatedtransfer stimuli, suggesting they are capable of generalising beyond the material they experience. However, on a more explicit task requiring them to categorise the displays, the autistic group performed significantly less accurately than the control group. Further research on the preservation of implicit learning skills among the autistic population, as well as the relationship between implicit and explicit knowledge (and specifically the activation of and access to implicit knowledge) could one day result in remedial strategies for the learning difficulties these individuals experience in typical educational and day-to-day settings.

\section{Therapy}

Implicit memory is known to be relatively more resistant to decay with age and not as affected by impairment as explicit memory. This fact has already been applied in therapy in the following methods:

Spaced retrieval - a technique by Landauer \& Bjork (1978) in which the information to be learned is retrieved repeatedly at gradually increasing delays. Camp et al. (2000) suggest that this relatively effortless method, in which retention intervals are increased only when success is achieved at a shorter interval, may rely on automatic, implicit memory.

Vanishing cues - by Glisky et al. (1986), a technique that involves gradually withdrawing or fading cue information until the target is produced in the absence of it (e.g., protect, protec_, pro , pr , $\mathrm{p}$

Errorless learning - by Baddeley \& Wilson (1994), consisting of eliminating negative evidence, so that the person can accumulate and consolidate only what is correct. 
Skills and expertise - applied by e.g., Zanetti et al., (2001), attempting to capitalise on retention and reactivation of formerly proceduralised skills, such as playing chess, typing, brushing teeth, and using the telephone.

The more we understand about the mechanism of implicit learning, and the conditions which are optimal for its occurrence as well as for the retrieval of the implicitly gained knowledge, the more successfully we can apply these findings in therapy and rehabilitation. ${ }^{20}$

The suggestions for therapy based on the research discussed in this dissertation are firstly that due attention should be paid to structuring training material appropriately. As mentioned in Chapter 1, DeKeyser (1995) failed to show implicit learning when he attempted to teach people multiple linguistic regularities, using input in which the evidence for these was spread out and intermixed. On the other hand, Williams (2005) presented his learners with material aiming for them just to learn one association, namely which particle co-occurs with animate and which with inanimate nouns. Arguably, participants could focus their attention more steadily on the target. Brain-training, although currently explored with relatively little accomplishment, might one day turn out to be more successful, especially when techniques based on implicit memory and learning are combined with linguistic material. It is known that implicit learning is related to language skills (see Chapter 1, section 1.1), and the possession of certain forms of linguistic knowledge (e.g. bilingualism) has been reported to help delay the onset of Alzheimer's Disease and reduce the severity of the symptoms (Craik, et al., 2010). Also, while in healthy aging it is noted that implicit memory is relatively preserved, Alzheimer's Disease affects both implicit and explicit memory. Exploring this fact could lead to the development of potential early diagnostics for Alzheimer's. This is an especially interesting avenue of research given that the aspect of knowledge which seems to be affected at the earliest stages of Alzheimer's is semantic knowledge, and specifically categorical knowledge based on generalisations rather than associative semantic knowledge. The potential of implicit memory and learning tests as a diagnostic will hopefully be explored when more is known about the individual differences in people's performance on the tasks.

\footnotetext{
${ }^{20}$ As Glisky \& Glisky (2002) suggest, the techniques which rely on implicit memory are primarily useful for individuals with severe impairments in explicit memory. For people with only mild memory deficits, such as those that occur in normal aging, using implicit processes may be less reliable than using residual explicit memory function.
} 
Little is known at the moment about the extent to which the procedures addressing implicit learning are sensitive and reliable enough to be a diagnostic tool. But this issue can be investigated, e.g., by looking at test - retest reliability or changing the task/rule so that it is a general semantic implicit learning that is tapped.

In all, implicit learning skills, language skills and memory condition all seem to be intricately related and the relationships between them still remain to be explored in detail. Investigating these relations and the potential applications of the implicit learning techniques might one day prove to be of considerable benefit to society.

\subsection{Conclusion}

Overall, the findings presented in this dissertation point to the conclusion that, contrary to the popular view of the mechanism underlying implicit learning, it is possible that it can lead to the formation of unconscious semantic regularities, not based on perceptual similarity to the training material. Researchers have only just begun to identify the factors which may influence implicit learning, including the role of the native language. Much more study is needed, but there is potential for the findings from this line of research to one day have important implications for psychological and linguistic theory, as well as practical applications both to pedagogy and therapy. 


\section{References}

Altmann, G. T. M., Dienes, Z., \& Goode, A. (1995). Modality Independence of Implicitly Learned Grammatical Knowledge. Journal Of Experimental Psychology. Learning Memory And Cognition. American Psychological Association.

Baars, B. J. (2002). The conscious access hypothesis: origins and recent evidence. Trends in cognitive sciences, 6(1), 47-52.

Baddeley, A. D., \& Wilson, B. A. (1994). When implicit learning fails: Amnesia and the problem of error elimination+ Neuropsychologia, 32, 53-68.

Bailey, T. M., \& Hahn, U. (2001). Determinants of wordlikeness: Phonotactics or lexical neighborhoods? Journal of Memory and Language, 44, 568-591.

Baese-Berk, M. (2010). An examination of the relationship between speech perception and production. Talk presented at Architectures and Mechanisms for Language Processing. York, UK.

Barsalou, L. W., Huttenlocher, J., \& Lamberts, K. (1998). Basing categorization on individuals and events. Cognitive psychology, 36(3), 203-72.

Batterink, L., \& Neville, H. (2011). Implicit and explicit mechanisms of word learning in a narrative context: an event-related potential study. Journal of Cognitive Neuroscience, 23, 3181-3196.

Berns, G. S., Cohen, J. D., \& Mintun, M. A. (1997). Brain regions responsive to novelty in the absence of awareness. Science, 276, 1272-1275.

Berns, M. S. (1984). Functional approaches to language and language teaching: Another look. Initiatives in communicative language teaching. A book of readings (pp. 3-21). Reading, MA: Addison-Wesley.

Berry, D. C., \& Broadbent, D. (1984). On the relationship between task performance and associated verbalisable knowledge. Quarterly Journal of Experimental Psychology, 36, 209-231.

Berry, D., \& Cock, J. (1998). Implicit Learning of Invariant Features? In M. A. Stadler \& P. A. Frensch (Eds.), Handbook of Implicit Learning (pp. 135-159). Thousand Oaks, California: SAGE Publications, Inc. 
Bickerton, D. (1999). Creole languages, the language bioprogram hypothesis, and language acquisition. In W. C. Ritchie \& T. K. Bhatia (Eds.), Handbook of child language acquisition. Academic Press.

Bjorklund, D. F., \& Jacobs, J. W. (1985). Associative and categorical processes in children's memory: The role of automaticity in the development of organization in free recall. Journal of Experimental Child Psychology, 39, 599-617.

Bolte, A., \& Goschke, T. (2008). Intuition in the context of object perception: intuitive gestalt judgments rest on the unconscious activation of semantic representations. Cognition, $108(3), 608-16$.

Brady, T. F., \& Oliva, A. (2008). Statistical learning using real-world scenes: extracting categorical regularities without conscious intent. Psychological science, 19(7), 678-85.

Braine, D. S., Brody, R. E., Brooks, P. J., Ross, A., Catalano, L., \& Fisch, S. M. (1990). Exploring Language Acquisition in Children with a Miniature Artificial Language : Effects of Item and Pattern Frequency, Arbitrary Subclasses, and Correction., 610, 591610.

Brainerd, C. J., Forrest, T. J., Karibian, D., \& Reyna, V. F. (2006). Development of the falsememory illusion. Developmental psychology, 42(5), 962-79.

Brainerd, C. J., \& Reyna, V. F. (2005). The science of false memory. New York: Oxford University Press.

Bright, J. E. H., \& Burton, A. M. (1994). Past midnight: semantic processing in an implicit learning task. The Quarterly journal of experimental psychology. A. Human experimental psychology, 47(1), 71-89.

Brockmole, J. R., Castelhano, M. S., \& Henderson, J. M. (2006). Contextual cueing in naturalistic scenes: Global and local contexts. Journal of experimental psychology. Learning, memory, and cognition, 32(4), 699-706.

Brockmole, J. R., Hambrick, D. Z., Windisch, D. J., \& Henderson, J. M. (2008). The role of meaning in contextual cueing: evidence from chess expertise. Quarterly journal of experimental psychology (2006), 61(12), 1886-96.

Brooks, L. R. (1978). Non-analytic concept formation and memory for instances. In E. Rosch \& B. Lloyd (Eds.), Cognition and concepts (pp. 169 - 211). Hillsdale, NJ: Erlbaum.

Brooks, P. J., Braine, M. D. S., Catalano, L., Brody, R. E., \& Sudhalter, V. (1993). Acquisition of Gender-like Noun Subclasses in an Artificial Language: The Contribution of Phonological Markers to Learning. Journal of Memory and Language, 32(1), 76-95.

Brown and P. Hagoort. (1993) The Processing Nature of the N400: Evidence from Masked Priming. Journal of Cognitive Neuroscience, 5(1). 
Brown, J., Aczel, B., Jimenez, L., Kaufman, S. B., \& Plaisted Grant, K. (2010). Intact Implicit Learning in Autism Spectrum Conditions. Quarterly Journal of Experimental Psychology, 69(3), 1789-1812.

Buchanan, L., Brown, N. R., Cabeza. R., \& Maitson, C. (1999). False memories and semantic lexicon arrangement. Brain and Language, 68(1-2), 172-177.

Camp, C. J., Bird, M. J., \& Cherry, K. E. (2000). Retrieval strategies as a rehabilitation aid for cognitive loss in pathological aging. In R. D. Hill, L. Bäckman, \& A. S. Neely (Eds.), Cognitive rehabilitation in old age (pp. 224-248). New York: Oxford University Press.

Carrión, R.E., Bly, B.M. (2007). Event-related potential markers of expectation violation in an artificial grammar learning task. Neuroreport 18, 191-195.

Chambers, K., Onishi, K., \& Fisher, C. (2003). Infants learn phonotactic regularities from brief auditory experience. Cognition, 56(3), 165-209.

Chang, F., Dell, G. S., \& Bock, K. (2006). Becoming Syntactic. Psychological Review, $113(2), 234-272$.

Chater, N., \& Christiansen, M. H. (2010). Language acquisition meets language evolution. Cognitive Science, 34, 1131-1157.

Chomsky, N. (1993). Language and Thought. London: Moyer Bell.

Craik F.I.M., Bialystok E., Freedman M. (2010). Delaying the onset of Alzheimer's disease: Bilingualism as a form of cognitive reserve. Neurology (75). 19. 1726-1729.

Christiansen, M. H., Conway, C. M., \& Onnis, L. (2012). Language and Cognitive Processes Similar neural correlates for language and sequential learning : Evidence from eventrelated brain potentials. Language and Cognitive Processes, 27(2), 231-256.

Christiansen, M. H., Louise Kelly, M., Shillcock, R. C., \& Greenfield, K. (2010). Impaired artificial grammar learning in agrammatism. Cognition, 116(3), 382-93.

Chun, M. (2000). Contextual cueing of visual attention. Trends in cognitive sciences, 4(5), $170-178$.

Chun, M. M. ., \& Jiang, Y. (1998). Contextual Cueing: Implicit Learning and Memory of Visual Context Guides Spatial Attention. Cognitive Psychology, 36(1), 28-71.

Chun, M. M., \& Jiang, Y. (1999). Top-Down Attentional Guidance Based on Implicit Learning of Visual Covariation. Psychological Science, 10(4), 360-365.

Cleary, A. M., \& Langley, M. M. (2007). Retention of the structure underlying sentences. Language and Cognitive Processes, 22(4), 614-628. 
Cleeremans, a, Destrebecqz, a, \& Boyer, M. (1998). Implicit learning: news from the front. Trends in cognitive sciences, 2(10), 406-16.

Cleeremans, A. Jiménez, L. (2002). Implicit learning and consciousness: A graded, dynamic perspective. In R.M. French, A. Cleeremans (Eds.), Implicit Learnind and Consciousness, Hove, UK: Psychology Press, pp. 1-40.

Cleeremans, A., \& Jimenéz, L. (1998). Implicit Sequence Learning. The Truth Is in the Details. In Stadler, Michael A. \& A. Frensch, Peter (Eds.), Handbook of Implicit Learning (pp. 323-364). Thousand Oaks, California: SAGE Publications, Inc.

Conway, C. M., Bauernschmidt, A., Huang, S. S., \& Pisoni, D. B. (2009). Implicit statistical learning in language processing: word predictability is the key. Cognition, 114(3), 35671.

Conway, C. M., \& Christiansen, M. H. (2006). Statistical learning within and between modalities: pitting abstract against stimulus-specific representations. Psychological science, 17(10), 905-12.

Conway, C. M., \& Pisoni, D. B. (2008). Neurocognitive basis of implicit learning of sequential structure and its relation to language processing. Annals of the New York Academy of Sciences, 1145, 113-31.

Craik, F. I. M. \& Lockhart R. S. (1972). "Levels of processing: A framework for memory research". Journal of Verbal Learning \& Verbal Behavior 11 (6): 671-84.

Craik, F.I.M., \& Tulving, E. (1975). Depth of processing and the retention of words in episodic memory. Journal of Experimental Psychology: General, 104, 268-294.

DeKeyser, R. M. (1995). Learning Second Language Grammar Rules. Studies in Second Language Acquisition, 17(03), 379-410.

Deese, J. (1959). On the prediction of occurrence of certain verbal intrusions in free recall. Journal of Experimental Psychology, 58, 17-22.

Dell, G. S., Reed, K. D., Adams, D. R., \& Meyer, a S. (2000). Speech errors, phonotactic constraints, and implicit learning: a study of the role of experience in language production. Journal of experimental psychology. Learning, memory, and cognition, 26(6), 1355-67.

Dienes, Z. (2008). Subjective measures of unconscious knowledge. Progress in Brain Research, 168, 49-64.

Dienes, Z., \& Altmann, G. (1997). Transfer of implicit knowledge across domains: How implicit and how abstract? In Dianne C. Berry (Ed.), How implicit is implicit learning? Oxford University Press.

Dienes, Z., \& Perner, J. (1999) A theory of implicit and explicit knowledge. Behavioural and Brain Sciences, 22, 735-755. 
Dienes, Z., \& Scott, R. (2005). Measuring unconscious knowledge: Distinguishing structural knowledge and judgment knowledge. Psychological Research, 69, 338 - 351.

Dominey, P. F., \& Ramus, F. (2000). Neural network processing of natural language: I. Sensitivity to serial, temporal and abstract structure of language in the infant. Language and Cognitive Processes, 15(87-127).

Eckman, F., \& Iverson, G. (in press). The Role of Native Language Phonology in the Production of L2 Contrasts. Studies in Second Language Acquisition. 35, 1-26.

Eich, J. (1982). A composite holographic associative recall model. Psychological Review, 89, $627-661$

Ellis, N. C. (1994). Vocabulary Acquisition: The Implicit Ins and Outs of Explicit Cognitive Mediation. In N. Ellis (Ed.), Implicit and explicit learning of languages. London: Academic Press.

Ellis, N. C. (2002). Frequency Effects in Language Processing: A Review with Implications for Theory of Implicit and Explicit Language Acquisition. Studies in Second Language Acquisition, 24, 143-188.

Ellis, N. C. (2003). Constructions, chunking, and connectionism: The emergence of second language structure. In C. Dougherty \& M. H. Long (Eds.), Handbook of second language acquisition (pp. 33-68). Oxford: Blackwell.

Ellis, N. C. (2005). At the interface: dynamic interactions of explicit and implicit language knowledge.Studies in Second Language Acquisition, 27(02), 305-352.

Ellis, N. C. (2001). Memory for language. In P. Robinson (Ed.), Cognition and second language instruction (pp. 33-68). Cambridge: Cambridge University Press.

Ellis, N. C. (2006). Language acquisition as rational contingency learning. Applied Linguistics, 27, 1-24.

Ellis, R. (2005). Measuring implicit and explicit knowledge of a second language: A psychometric study. Studies in Second Language Acquisition, 27, 141-172.

Evans, J. L., Saffran, J. R., \& Robe-Torres, K. (2009). Statistical learning in children with specific language impairment.. Journal of Speech, Language, and Hearing Research, $52,321-335$.

Feldman, N., Myers, E., White, K., Griffiths, T., \& Morgan, J. (2011). Learners use wordlevel statistics in phonetic category acquisition. Proceedings of the 35th Boston University Conference on Language Development.

Ferrer-Gil, E. (1994). La unidad de aprendizaje en procesamiento no consciente: Un Analisis experimental [The unit of learning in unconscious processing: An experimental analysis]. Psycologemas, 7, 195-217. 
Firth, J. R. (1957). Modes of Meaning. Papers in Linguistics 1934-1951 (pp. 190-215). Oxford: Oxford University Press.

Frensch, P. A. (1998). One Concept, Multiple Meanings: On How to Define the Concept of Implicit Learning. Handbook of Implicit Learning (pp. 47-104). Thousand Oaks, California: SAGE Publications, Inc.

Froehlich A.L., Anderson J.S, Bigler E.D., Miller J.S., Lange N.T., Dubray M.B., Cooperrider JR, Cariello A, Nielsen J.A, Lainhart J.E., (2012). Intact Prototype Formation but Impairred Generalization in Autism. Research in Autism Spectrum Disorders 6 . 921-930.

Gabrieli J.D.E, Cohen N.J, Corkin S. (1988). The impaired learning of semantic knowledge following bilateral medial temporal-lobe resection. Brain Cogn 7: 151-177.

Gladwell, M. (2008). Outliers: The Story of Success. New York: Little, Brown and Company.

Glisky E.L., \& Glisky, M.L. (2008). Memory rehabilitation in older adults. Cognitive Neurorehabilitaion, Second Edition: Evidence and Application, ed. Donald T. Suss, Gordon Winocur and Ian H. Robertson. Cambridge: Cambridge University Press.

Glisky E. L., Schacter D. L., \& Tulving E. (1986) Learning and retention of computerrelated vocabulary in memory-impaired patients: Method of vanishing cues. Journal of Clinical Experimental Neuropsychology: 8: 292-312.

Glosser, G., Friedman, R. B., Grugan, P. K., Lee, J. H., \& Grossman, M. 1998. Lexical semantic priming and associative priming in Alzheimer's disease. Neuropsychology, $12(2), 218-224$.

Gomez, R. L., Gerken, L., \& Schvaneveldt, R. W. (2000). The basis of transfer in artificial grammar learning. Memory \& Cognition, 28(2), 253-263.

Goschke, T., \& Bolte, A. (2007). Implicit learning of semantic category sequences: responseindependent acquisition of abstract sequential regularities. Journal of experimental psychology. Learning, memory, and cognition, 33(2), 394-406.

Goujon, A. (2011). Categorical implicit learning in real-world scenes: Evidence from contextual cueing. Quarterly journal of experimental psychology (2006), 64(5), 920-41. doi:10.1080/17470218.2010.526231

Goujon, A., Didierjean, A., \& Marmèche, E. (2007). Contextual cueing based on specific and categorical properties of the environment. Visual Cognition, 15(3), 257-275.

Guillelmon, D., \& Grosjean, F. (2001). The gender marking effect in spoken word recognition: The case of bilinguals. Memory \& Cognition, 29(3), 503-511.

Guo, X., Zheng, L., Zhu, L., Yang, Z., Chen, C., Zhang, L., Ma, W., \& Dienes, Z. (2011). Acquisition of conscious and unconscious knowledge of semantic prosody. Consciousness \& Cognition, 20, 417-425. 
Hama, M., \& Leow, R. P. (2010). Learning without awareness revisited. Studies in Second Language Acquisition, 32, 465-491.

Hay, J. F., \& Saffran, J. R. (2011). Rhythmic Grouping Biases Constrain Infant Statistical Learning. Infancy.

Hintzman, D. (1986). "Schema abstraction" in a multiple-trace memory model. Psychological Review, 93, 411-428.

Hoey, M. (2005). Lexical Priming: A New Theory of Words and Language. London: Routlege.

Howard, J. H., Howard, D. V., Japikse, K. C., \& Eden, G. F. (2006). Dyslexics are impaired on implicit higher-order sequence learning, but not on implicit spatial context learning. Neuropsychologia, 44(7), 1131-44.

Hudson Kam, C. L. (2009). More than words: Adults learn probabilities over categories and relationships between them. Language Learning and Development, 5(2), 115-145.

Hutchison, K.A., \& Balota, D.A. (2005). Decoupling semantic and associative information in false memories: Explorations with semantically ambiguous and unambiguous critical lures. Journal of Memory and Language, 52, 1-28.

Isemonger, I. M. (2007). Operational definitions of explicit and implicit knowledge: Response to R. Ellis (2005) and some recommendations for future research in this area. Studies in Second Language Acquisition, 29, 101-118.

Iwasaki, N. (2003). L2 Acquisition of Japanese: Knowledge and Use of Case Particles in SOV and OSV Sentences. Word Order and Scrambling (pp. 273 -300). Oxford: Blackwell.

Jimenez-Fernandez, Gracia Vaquero, J. M. M., Jimenez, L., \& Defior, S. (2011). Dyslexic Children Show Deficits in Implicit Sequence Learning, but Not in Explicit Sequence Learning or Contextual Cueing. Annals of Dyslexia, 61(1), 85-110.

John, C., \& McCarthy, D. (2000). Word Sense Disambiguation Using Automatically Acquired Verbal Preferences. Computers and the Humanities, 34(1/2), 109 - 114.

Johnstone, T., \& Shanks, D. R. (1999). Two mechanisms in implicit artificial grammar learning? Comment on Meulemans and Van der Linden (1997). Journal of Experimental Psychology: Learning, Memory, and Cognition., 25, 524-531.

Kaufman, S. B., Deyoung, C. G., Gray, J. R., Jiménez, L., Brown, J., \& Mackintosh, N. (2010). Implicit learning as an ability. Cognition, 116(3), 321-40.

Kiefer, M. (2002). The N400 is modulated by unconsciously perceived masked words: further evidence for an automatic spreading activation account of N400 priming effects. Brain research. Cognitive brain research, 13(1), 27-39. 
Kiyokawa, S., Dienes, Z., Tanaka, D., Yamada, A., \& Crowe, L. (2012). Cross cultural differences in unconscious knowledge. Cognition, 124(1), 16-24.

Knowlton, B J, \& Squire, L. R. (1996). Artificial grammar learning depends on implicit acquisition of both abstract and exemplar-specific information. Journal of experimental psychology. Learning, memory, and cognition, 22(1), 169-81.

Knowlton, Barbara J., Ramus, S. J., \& Squire, L. R. (1992). Intact Artificial Grammar Learning in Amnesia: Dissociation of Classification Learning and Explicit Memory for Specific Instances. Psychological Science, 3(3), 172 - 179.

Krashen, S. D. (1981). Second Language Acquisition and Second Language Learning. Oxford: Pergamon.

Lambert, A. J., \& Sumich, A. L. (1996). Spatial orienting controlled without awareness: a semantically based implicit learning effect. The Quarterly journal of experimental psychology. A, Human experimental psychology, 49(2), 490-518.

Lambert, A., Naikar, N., McLachlan, K., \& Aitken, V. (1999). A new component of visual orienting: Implicit effects of peripheral information and subthreshold cues on covert attention. Journal of Experimental Psychology: Human Perception and Performance, $25(2), 321-340$.

Lambert, T. (2003). Visual orienting, learning and conscious awareness. In L. Jiménez (Ed.), Attention and implicit learning (pp. 253 -275). Amsterdam: Benjamins.

Landauer, T. K., \& Bjork, R. A. (1978). Optimum rehearsal patterns and name learning. In M. M. Gruneberg, P. E. Morris, \& R. N. Sykes (Eds.), Practical aspects of memory(pp. 625-632). London: Academic Press.

Langdon, M. (1984). Noise words in Guaranı'. In Hinton, L., Nichols, J., \& Ohala, J. (Eds.), Sound symbolism (pp. 94-103). Cambridge, England: Cambridge University Press.

Lany, J., \& Saffran, J. R. (2011). Interactions between statistical and semantic information in infant language development. Developmental science, 14(5), 1207-19.

Leow, Ron. (2000). A study of the role of awareness in foreign language behavior: Aware vs. unaware learners. Studies in Second Language Acquisition, 22, 557-584.

Leow, Ronald. (1997). Attention, awareness, and foreign language behaviour. Language Learning, 47, 567 - 506.

Leung, J. H. C., \& Williams, J. N. (2011). The Implicit Learning of Mappings Between Forms and Contextually Derived Meanings. Studies in Second Language Acquisition, $33(01), 33-55$.

Leung, J. H. C., \& Williams, J. N. (2012). Constraints on Implicit Learning of Grammatical Form-Meaning Connections. Language Learning, 62(2), 634-662. 
Lewicki, P, Hill, T., \& Bizot, E. (1988). Acquisition of procedural knowledge about a pattern of stimuli that cannot be articulated. Cognitive psychology, 20(1), 24-37.

Lewicki, Pawel. (1986). Processing Information About Covariations That Cannot Be Articulated. Journal of Experimental Psychology: I .earning. Memory, and Cognition, 12(1), 135-146.

Lewicki, Pawel, Czyzewska, M., \& Hoffman, H. (1987). Unconscious acquisition of complex procedural knowledge. Journal of Experimental Psychology: Learning, Memory, and Cognition, 13(4), 523-530.

Lotz, A., \& Kinder, A. (2006). Transfer in artificial grammar learning: the role of repetition information. Journal of experimental psychology. Learning, memory, and cognition, 32(4), 707-15.

Lovibond, P. F., \& Shanks, D. R. (2002). The role of awareness in Pavlovian conditioning: Empirical evidence and theoretical implications. Journal of Experimental Psychology: Animal Behavior Processes, 28(1), 3-26.

Lund, K., \& Burgess, C. (1996). Producing high-dimensional semantic spaces from lexical cooccurrence. Behavior Research Methods, Instruments, \& Computers, 28, 203-208.

Manza, L., \& Reber, A. S. (1997). Representing artificial grammars: Transfer across stimulus forms and modalities. In Dianne C. Berry (Ed.), How implicit is implicit learning? Debates in psychology. (pp. 73 - 106). New York, NY, US: Oxford University Press.

Marcus, G. F., Vijayan, S., Bandi Rao, S., \& Vishton, P. M. (1999). Rule learning by sevenmonth-old infants. Science (New York, N.Y.), 283(5398), 77-80.

Mathews, R. C., Buss, R. R., Stanley, W. B., Blanchard-fields, F., \& Cho, J. R. (1989). Role of Implicit and Explicit Processes in Learning From Examples : A Synergistic Effect, 15(6), 1083-1100.

Maye, J., \& Gerken, L. (2000). Learning phonemes without minimal pairs. In S. C. Howell, S. A. Fish, \& T. Keith-Lucas (Eds.), Proceedings of the 24th Annual Boston University Conference on Language Development (pp. 522-533).

Maye, J., Werker, J. F., \& Gerken, L. (2002). Infant sensitivity to distributional information can affect phonetic discrimination. Cognition, 82(3), B101-11.

McEvoy, C. L., Nelson, D. L., \& Komatsu, T. (1999). What is the connection between true and false memories? The differential roles of iteritem associations in recall and recognition. Journal of Experimental Psychology:Learning, Memory, and Cognition, 25, 1177-1194.

McGeorge, P., \& Burton, A. M. (1990). Semantic processing in an incidental learning task. The Quarterly Journal of Experimental Psychology Section A, 42(3), 597-609. 
Minshew, N. J., Meyer, J., \& Goldstein, G. (2002). Abstract reasoning in autism: A dissociation between concept formation and concept identification. Neuropsychology, $16,327-334$.

Misyak, J. B., Christiansen, M. H., \& Tomblin, J. B. (2010). On-line individual differences in statistical learning predict language processing. Frontiers in psychology, 1, 31.

Misyak, J. B., Goldstein, M. H., \& Christiansen, M. H. (n.d.). Statistical-sequential learning in development. Statistical learning and language acquisition. Berlin: Mouton de Gruyter.

Monaghan, P., Christiansen, M. H., \& Fitneva, S. a. (2011). The arbitrariness of the sign: learning advantages from the structure of the vocabulary. Journal of experimental psychology. General, 140(3), 325-47.

Morgan, J. L., Meier, R. P., \& Newport, E. L. (1989). Facilitating the acquisition of syntax with cross-sentential cues to phrase structure. Journal of Memory and Language, (28), $67-85$.

Morgan-Short, K., Steinhauer, K., Sanz, C., \& Ullman, M. (2007). Explicit and implicit second language training differentially affect the achievement of native-like brain activation patterns. Journal of Cognitive Neuroscience., 24, 933-947.

Neely, J. (1977). Semantic Priming and Retrieval from Lexical Memory: Roles of Inhibitionless Spreading Activation and Limited-Capacity Attention. Journal of Experimental Psychology: General, 106(226-254).

Nelson, D. L., Bennet, D. J., \& Leibert, T. W. (1997). One step is not enough: Making better use of association norms to predict cued recall. Memory \& Cognition, 25, 785-796.

Newell, B. R., \& Bright, J. E. H. (2002). Well past midnight: Calling time on implicit invariant learning? European Journal of Cognitive Psychology, 14(2), 185-205.

Nygaard, L. C., Cook, A. E., \& Namy, L. L. (2009). Sound to meaning correspondences facilitate word learning. Cognition, 112(1), 181-6.

Ober B.A., Shenaut G.K., Reed B.R. (1995). Assessment of associative relations in Alzheimer's disease: evidence for preservation of semantic memory. Aging and Cognition, 2 (4): 254-67.

Onnis, L., Edelman, S., \& Waterfall, H. R. (2011). Local statistical learning under crosssituational uncertainty. In L. Carlson, C. Hölscher, \& T. Shipley (Eds.), Proceedings of the 33rd Annual Meeting of the Cognitive Science Society Conference.

Onnis, L., Waterfall, H. R., \& Edelman, S. (2008). Learn locally, act globally: learning language from variation set cues. Cognition, 109(3), 423-30.

Ozonoff, S., \& Miller, J. N. (1995). Teaching theory of mind: A new approach to social skills training for individuals with autism. Journal of Autism and Developmental Disorders, 


\section{$25,415-433$.}

Pacton, S., Perruchet, P., Fayol, M., \& Cleeremans, A. (2011). Implicit learning out of the lab the case of orthographic regularities. Journal Of Experimental Psychology. General., $130(3), 401-426$.

Paradis, M. (2004). A neurolinguistic theory of bilingualism. Amsterdam: John Benjamins.

Peperkamp, S., Le Calvez, R., Nadal, J.-P., \& Dupoux, E. (2006). The acquisition of allophonic rules: statistical learning with linguistic constraints. Cognition, 101(3), B3141 .

Perruchet, P. (1994). Learning from complex rule-governed environments: On the proper functions of nonconscious and conscious processes. In C. Umiltà \& Morris Moscovitch (Eds.), Attention and performance $X V$ : Conscious and nonconscious information processing (pp. 811-835). Cambridge, MA: MIT Press.

Perruchet, P. (2008). Implicit Learning. In J. Byrne (Ed.), Cognitive psychology of memory. Vol.2 of Learning and memory: A comprehensive reference (pp. 597-621). Oxford: Elsevier.

Perruchet, P., \& Pacteau, C. (1990). ynthetic grammar learning: Implicit rule abstraction or explicit fragmentary knowledge. Journal of Experimental Psychology: General, 119(3), $264-275$.

Perruchet, P., \& Pacton, S. (2006). Implicit learning and statistical learning: one phenomenon, two approaches. Trends in cognitive sciences, 10(5), 233-8.

Perruchet, P., \& Vinter, A. (1998). PARSER: A Model for Word Segmentation. Journal of Memory and Language, 39(2), 246-263.

Perruchet, Pierre, Gallego, J., \& Savy, I. (1990). A critical reappraisal of the evidence for unconscious abstraction of deterministic rules in complex experimental situations. Cognitive Psychology, 22(4), 493-516.

Plante, E., Gomez, R., \& Gerken, L. (2002). Sensitivity to word order cues by normal and language/learning disabled adults. Journal of Communication Disorders, 35(5), 453462.

Plaut, D., \& Shallice, T. (1993). Deep dyslexia: A case study of connecionist neuropsychology. Cognitive Neuropsychology, 10, 377-500.

Pothos, E. M. (2005). Expectations about stimulus structure in implicit learning. Memory \& cognition, 33(1), 171-81.

Pothos, E. M., \& Bailey, T. M. (2000). The role of similarity in artificial grammar learning. Journal of Experimental Psychology: Learning, Memory, and Cognition, 26(4), 847862. 
Rauch, S. L., Savage, C. R., Brown, H. D., Curran, T., Alpert, N. M., Kendrick, A., Fischman, A. J., et al. (1995). A PET investigation of implicit and explicit sequence learning. Human Brain Mapping, 3(4), 16.

Reber, A. S. (1967). Implicit learning of artificial grammars. Journal of Verbal Learning and Verbal Behavior, 6(6), 855-863.

Reber, A. S. (1969). Transfer of syntactic structure in synthetic languages. Journal of Experimental Psychology, 82, 115-119.

Reber, A. S., \& Allen, R. (1978). Analogic and abstraction strategies in synthetic grammar learning: A functionalist interpretation. Cognition, 6, 189-221.

Reber, A. S. (1989). Implicit learning and tacit knowledge. Journal of Experimental Psychology: General, 118(3).

Rebuschat, P., Hamrick, P., Sachs, R., Ziegler, N., \& Riestenberg, K. (in preparation). Awareness, reactivity and subjective measures: A contribution to the Williams-Leow debate.

Rebuschat, P., \& Williams, J. N. (2009). Implicit Learning of Word Order. In N. A. Taatgen \& H. Van Rijn (Eds.), Proceedings of the 31th Annual Conference of the Cognitive Science Society (pp. 425-430). Austin, TX: Cognitive Science Society.

Rebuschat, Patrick, \& Williams, J. N. (2011). Implicit and explicit knowledge in second language acquisition. Applied Psycholinguistics, FirstView, 2-28.

Resnick, P. (1997). Selectional preference and sense disambiguation. Proceedings of ACL Singlex Workshop on Tagging Text with Lexical Semantics, Why, What and How?

Roediger, H. L. III, Balota, D. A., \& Watson, J. M. (2001). Spreading activation and arousal of false memories. In H. L. Roediger III, J. S. Nairne, I. Neath, \& A. M. Surprenant (Eds.), The nature of remembering: Essays in honor of Robert G. Crowder (pp. 95 115). Washington, DC: American Psychological Association.

Roediger, H. L. I., Watson, J. M., McDermott, K. B., \&, \& Gallo, D. A. (2001). Factors that determine false recall: A multiple regression analysis. Psychonomic Bulletin and Review, 8, 385-407.

Roediger, H. L. III, McDermott, K. B. (1995). Creating false memories: Remembering words not presented on lists. Journal of Experimental Psychology: Learning, Memory, and Cognition, 21, 803-814.

Ropar, D., \& Peebles, D. (2007). Sorting preference in children with autism: The dominance of concrete features. Journal of Autism and Developmental Disorders, 37, 270-280.

Rosa, E., \& Leow, R. P. (2004). Awareness, different learning conditions, and second language development. Applies Psycholinguistics, 25, 269-292. 
Saffran, J. R. (2003). Statistical language learning: mechanisms and constraints. Current Directions in Psychological Science, 12(4), 110-114.

Saffran, J. R., Newport, E. L., \& Aslin, R. N. (1996-a). Statistical learning in 8-month-old infants. Science, 274, $1926-1928$.

Saffran, J. R., Newport, E. L., \& Aslin, R. N. (1996-b). Word segmentation: The role of distributional cues. Journal of Memory and Language, 35, 606-621.

Saffran, J. R., \& Wilson, D. P. (2003). From Syllables to Syntax: Multilevel Statistical Learning by 12-Month-Old Infants. Infancy, 4(2), 273-284.

Schankin, A., Hagemann D. \& Danner D., (2011). "Violations of implicit rules elicit an early negativity in the event-related potential." Neuroreport 22(13): 642-645.

Schmidt, R. W. (1995). Consciousness and foreign language learning: A tutorial on the role of attention and awareness in learning. In R.Schmidt (Ed.), Attention and awareness in foreign language.learning (pp. 1-63). Honolulu: University of Hawai'i Press.

Schmidt, R. W. (1990). The Role of Consciousness in Second Language Learning. Applied Linguistics, 11(2), 129-158. doi:10.1093/applin/11.2.129

Schmidt, Richard W, \& Frota, S. (1986). Developing basic conversational ability in a second language: a case study of an adult learner of Portugese. In R. R. Day (Ed.), Talking to learn: Conversation in second language acquisition (pp. 237-326). Rowley, MA: Newbury House.

Schmitt, N. (2010). Researching Vocabulary: A Vocabulary Research Manual. Palgrave Press.

Scott, R. B., \& Dienes, Z. (2008). The conscious, the unconscious, and familiarity. Journal of experimental psychology. Learning, memory, and cognition, 34(5), 1264-88.

Scott, R., \& Dienes, Z. (2010). Knowledge applied to new domains: the unconscious succeeds where the conscious fails. Consciousness and Cognition, 19, 391-398.

Seger, C. (1998). Multiple forms of implicit learning. Handbook of Implicit Learning (pp. 295-320). Thousand Oaks, California: SAGE Publications, Inc.

Servan-Schreiber, D Anderson, J. R. (1990). Learning artificial grammars with competitive chunking. Journal of Experimental Psychology: Learning, Memory, and Cognition, 16, 592-608.

Shanks, D.R., (2005). Implicit learning. In: Lamberts, K., Goldstone, R. (Eds.), Handbook of Cognition. Sage, London, pp. 202-220.

Shanks, D. R., \& St. John, M. F. (1994). Characteristics of dissociable human learning systems. Behavioral and Brain Sciences, 17, 367-395. 
Schulman A. I. (1974). Memory for words recently classified. Memory \& Cognition, 2.47-52.

Sinclair, J. (1996). The search for units of meaning. Textus: English Studies in Italy, 9, 75106.

Sinclair, J. M. (1991). Corpus, Concordance, Collocation. Oxford: Oxford University Press.

Sinclair, J. M. (2004). Trust the Text: Lexis, Corpus, Discourse. London: Routlege.

Siyanova-Chanturia, A., Conklin, K., \& Schmitt, N. (2011). Adding more fuel to the fire: An eye-tracking study of idiom processing by native and nonnative speakers. Second Language Research, 27, 251-272.

Smith, L. \& Yu, C. (2008). Infants rapidly learn word-referent mappings via crosssituational statistics. Cognition 106(3): 1558-1568.

Stadler, M. A. (1989). On learning complex procedural knowledge. Journal of Experimental Psychology: Learning, Memory and Cognition, 15, 1061 - 1069.

Stroop, J. R. (1935). Studies of interference in serial verbal reactions. Journal of Experimental Psychology, 18, 643-622.

Thompson, S. P., \& Newport, E. L. (2007). Statistical Learning of Syntax: The Role of Transitional Probability. Language Learning and Development, 3(1), 1-42.

Tunney, R J, \& Altmann, G. T. (2001). Two modes of transfer in artificial grammar learning. Journal of experimental psychology. Learning, memory, and cognition, 27(3), 614-39.

Tunney, Richard J, \& Shanks, D. R. (2003). Subjective measures of awareness and implicit cognition. Memory \& cognition, 31(7), 1060-71.

Tzelgov, J. (1997). Specifying the relations between automaticity and consciousness: A theoretical note. Consciousness and Cognition, 6, 441-451.

Van Opstal, F., de Lange, F. P., \& Dehaene, S. (2011). Rapid parallel semantic processing of numbers without awareness. Cognition, 120(1), 136-47.

Van Sommers,P. (1984). Drawing and Cognition.Cambridge, UK: Cambridge University Press.

Vinter, A., \& Perruchet, P. (2000). Implicit learning in children is not related to age: Evidence from drawing behavior. Child Development, 71(5), 1223-1240.

Vinter, A., \& Perruchet, P. (1999). Isolating unconscious influences: The neutral parameter procedure.Quarterly Journal of Experimental Psychology, 52A(4), 857-875.

Vokey, J. R., \& Brooks, L. R. (1992). Salience of item knowledge in learning artificial grammars. Journal of Experimental Psychology: Learning, Memory, and Cognition, $18(2), 328-344$. 
Warker, J. a, \& Dell, G. S. (2006). Speech errors reflect newly learned phonotactic constraints. Journal of experimental psychology. Learning, memory, and cognition, 32(2), 387-98.

White, L. (2003). Second language acquisition and universal grammar. Cambridge: Cambridge University Press.

Williams, J. (2012). Implicit adult language learning: Prospects and limitations. Talk given to the Department of Linguistics (Vol. Talk given). United Arab Emirates University.

Williams, J. N. (2003). Inducing abstract linguistic representations: human and connectionist learning of noun classes. In R. van Hout, A. Hulk, F. Kuiken, \& R. Towell (Eds.), The Lexicon-Syntax Interface in Second Language Acquisition (pp. 151-174). Amsterdam: John Benjamins.

Williams, J. N. (2005). Learning Without Awareness. Studies in Second Language Acquisition, 27(02), 269-304.

Williams, J. N., \& Kuribara, C. (2008). Comparing a nativist and emergentist approach to the initial stage of SLA: An investigation of Japanese scrambling. Lingua, 118(4), 522-553.

Williams, N. (2011). Single-trial analysis of Event-Related Potentials (ERPs). University of Reading.

Willingham, D. B., Nissen, M. J., \& Bullemer, P. (1989). On the development of procedural knowledge. Journal of Experimental Psychology: Learning, Memory, and Cognition, 15, $1047-1060$.

Wonnacott, E., Boyd, J. K., Thomson, J., \& Goldberg, A. E. (2012). Input effects on the acquisition of a novel phrasal construction in 5year olds. Journal of Memory and Language, 66(3), 458-478.

Wonnacott, E., Newport, E., \& Tanenhaus, M. (2008). Acquiring and processing verb argument structure: distributional learning in a miniature language. Cogn Psychol, 56, $165-209$.

Wright, R. L., \& Burton, A. M. (1995). Implicit learning of an invariant: Just say no. Quarterly Journal of Experimental Psychology, 48, 783-796.

Wright, Richard L., \& Whittlesea, B. W. A. (1998). Implicit learning of complex structures: active adaptation and selective processing in acquisition and application. Memory \& cognition, 26(2), 402-420.

Yee, E., Huffstetler, S., \& Thompson-Schill, S.L. (2011). Function follows form: Activation of shape and function features during object identification. Journal of Experimental Psychology:General,140(3), 348-363.

Zanetti O, Zanieri G, Di Giovanni G et al. (2001). Effectiveness of procedural memory 
stimulation in mild Alzheimer's disease patients: a controlled study. Neuropsychological Rehabilitation. 11:263-272. 


\section{APPENDIX A: Example questionnaires from Experiment 1}

\section{Example training questionnaire (Note: Answer scale only shown for the first item here)}

I am testing the relation between the domain of information and people's judgement of importance. You will see 50 extracts from newspapers about politics, health, environment and entertainment. Please mark how important the information is to you on a scale from 0 to 5; 0 for not important, 5 for very important. Thank you for your participation.

1. Research shows that having strong ties to co-workers can help mitigate job-related stress - a good reason to make some office buddies.

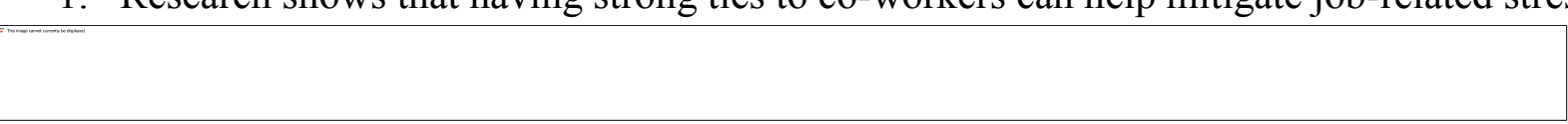

2. Supermodel Naomi Campbell has been held at Heathrow Airport on suspicion of assaulting a police officer.

3. A well known CNN journalist Alison Smith lost her job as a news presenter after she arbitrarily truncated the text which had been written for her and added her own improper commentary of the events.

4. The British have long tried to diminish the input of the two Polish mathematicians into the breaking of the code of Enigma, when in fact it was them who provided the crucial contribution.

5. A high number of fires have occurred in Hawaii over the last couple of years and had a very damaging effect on the environment. They not only devour the forests, but also deplete nitrogen from Hawaii's already nitrogen-poor soils.

6. Polish President Lech Kaczynski and club officials pledged to battle hooliganism after more than 200 fans rioted after Legia Warsaw won the Polish League title. "The events greatly damaged our reputation," Kaczynski said. "Such events are extremely dangerous, and we will fiercely fight them." 
7. So far, about 1,100 American children have lost a parent in Iraq, and hundreds of other kids have had parents killed in Afghanistan. We believe the U.S. government should pay college education costs for those children.

8. Al Gore remarked that suggestions that a global warming is a relatively temporary phenomenon, diminish the force of the alarming calls for a global action.

9. Writing a thesis is a laborious process which often surprises you even as you write it. You'll see your ideas changing and developing and so sometimes you'll want to truncate one section to make space to write more in another or even delete some parts altogether.

10. An Australian man jumped onto the back of a crocodile that was attacking his wife, and forced it to flee.

11. The body must first deplete carbohydrates before it can begin to burn fat.

12. Renamo says all its equipment - solar-powered radios and laptop computers - were paid for by a conservative Louisiana businessman.

13. It has been argued that faith, especially believing that the deceased go to a better place and one day we will all be reunited in heaven does indeed mitigate the grief caused by the death of a loved one.

14. Food today travels between 1,500 and 2,500 miles from farm to market.

15. What many internet users don't know is that you don't always need to send the long string of letters as a link to something you've found. You can truncate the website URL by using certain simple techniques.

16. A White House conference on global warming opens tomorrow in Washington. Representatives from 17 nations will participate in the two day meeting. 
17. Meaning of your Tarot Cards: "The Fool" represents the dreamer in you, the idealist, the mystic. If this card is accompanied by "The Devil", the latter may diminish the influence of the former.

18. How to stay healthy as a vegetarian? Losing meat and falling back on fatty, sugary snacks is a common mistake. You or other vegetarians in your family need a diet rich in veggies and fruits, beans, nuts and seeds.

19. The American vision of the Holocaust continues to insist that the victims have not died in vain, trying to depict hope and justice and the future as a victory that will mitigate despair.

20. According to our survey, nearly three quarters of families plan to cut their spending this year.

21. The loss of the Amazon rainforest would not deplete the oxygen on Earth, that's not the issue, rather it would increase the amount of carbon dioxide which traps the earth's heat like a glass in a greenhouse.

22. There must be a bug in the programme script: if you write a "twitter" command message that contains the word "as", it truncates the message at that point in the command preview. It doesn't offer any alternatives to allow you to treat "as" like a normal word.

23. Leo: Although your home life may be on your mind much of the day, it does not stop you from your work. You find yourself ambitious and inventive most of the day.

24. The fact that TV is still the most popular medium does not diminish the significance of the radio in shaping public opinion.

25. Sleepwalking becomes a problem when it disturbs your sleep so much that you're tired during the day or if it's so extreme that you become a threat to someone or to yourself. 
26. Older people's lives are frequently disrupted by loss and change; companion animals can mitigate the effects of loss and ease times of stressful transitions, such as retirement.

27. Environmental degradation poses a threat for the developing countries which depend on the environment for their economic growth. The situation is worst in the poorest countries where poverty and population pressure lead people to deplete the natural resources to satisfy their immediate needs.

28. The economic crisis has visibly hit the university graduates who are just entering the workforce. Boston_Consulting among many companies had to severely truncate the lists of accepted interns, leaving many hopeful youngsters jobless.

29. If you have to add some flavor to your vegetables, at least pick the right one. Top your salad with one tablespoon of heart-healthy olive oil plus as much red wine or balsamic vinegar as you want.

30. The spelling mistakes the protesters made on their banners like "kill the criminal PERSIDENT" or "we want PEACE. No more war" didn't diminish the prominence of the protest and even foreign newspapers reported the incident.

31. But if God will neither take away our suffering nor mitigate it, we pray that God will at least reveal the reasons for sending it.

32. In the newest version of the programme, the RichTextBox will truncate the line at the end. You can see the characters when you highlight the line, but not in normal mode.

33. The relationship between serotonin and depression was discovered 25 years ago when a drug called reserpine was observed to deplete serotonin, the chemical responsible for our mood, and cause depression in a large number of patients.

34. President Obama's faux pas about the mixed race dogs or disabled Olympics didn't in the slightest bit diminish his greatness in the eyes of Americans. If it had been president Bush who had said it, he would have been virtually lynched. 
35. 40\% of Americans approve of President Bush's job performance, according to the latest FOX News poll. And only 32 percent approve of the Democratic leadership.

36. Ian Halcrow's Macbeth looks great but never draws us close, and he and others swallow or truncate their sentences as if they cared very little for them.

37. When you suffer from one of those severe migraines use zinc to mitigate the pain as it usually works.

38. CFS is just one chemical that depletes the ozone layer, which is not something we can renew.

39. The presence in the Vatican of Polish Pope John-Paul II was an important influence on the Solidarity movement throughout the 1980s.

40. The public obviously has had enough of the prime minister trying to diminish the status of the president and vice versa.

41. Allergy UK is the country's leading national medical charity dealing with allergy and we are here to help you. We can provide you with up to date information on all aspects of allergy.

42. Your movie file is too large to fit on one disk? You'll need to truncate it then. You do this in the timeline view of your movie editor.

43. Nothing, not even family support can really mitigate the agony of a HIV sufferer who feels the cold breath of death on his shoulder day by day.

44. In Nepal, 120,000 trekkers a year deplete forests by always using wood in lodges or houses for baths and cooking. Many forest dwelling animals are facing extinction as a result. 
45. Get out, meet people, and be open to new people and new experiences. You'll meet someone. After all, dating is a process of elimination -you just haven't met the right guy yet.

46. Many new political philosophies diminish the role of the state in human development by saying it hardly plays a role at all.

47. Secondary school pupils are to be mentored by university students to encourage them to continue their education to degree level.

48. As Oprah Winfrey said: "if you want your life to be more rewarding, you have to change the way you think".

49. New research at the University of Toronto found that probiotics, "good bacteria" taken in pill or powder form, appear to mitigate depression and anxiety.

50. Could it be possible that millions of people are taking medications that unknowingly deplete essential nutrients from their bodies, which in turn creates more health problems? 


\section{Example test questionnaire from Experiment 1}

Note: Answer boxes only shown for the first item

This time I would like you to:

1) mark whether you think the distinguished WORD in each sentence is used correctly or not;

2) mark how confident you are on a 1-5 scale: $1=$ not confident at all, $5=100 \%$ confident;

3) tell me what you used in determining your choice; was it:

a guess = you could just as well flip a coin,

- intuition = you think $x$ is correct, but you could not tell why

- your own definition,

- memory of a similar sentence

- you knew what the word means before this experiment.

Thank you for your participation.

1. Posting departure times at bus stops should MITIGATE frustration at long intervals between buses, because people will know when the bus will arrive and find other ways to spend their time rather than simply wait for the bus.

\begin{tabular}{|l|l|}
\hline \multicolumn{2}{|c|}{ Is the word } \\
correct? \\
\hline YES & NO \\
\hline
\end{tabular}

\begin{tabular}{|l|}
\hline How did you mark your answer? \\
\hline - by guessing \\
- intuition \\
- I formulated a definition and used it \\
- I remembered a similar sentence \\
- I knew before what this word means \\
\hline
\end{tabular}


2. More trees are being planted in many tropical regions in an effort to TRUNCATE the dramatic effects of deforestation and to ensure the availability of forest resources for future generations.

3. A recent study led by UCSB earth scientists has uncovered single-celled organisms that DEPLETE the oil supply as they devour its sources underneath the sea floor.

4. The new domain will TRUNCATE all passwords to exactly 12 characters, so if yours was longer - now it will only consist of the first 12 symbols.

5. There are thousands of ways to MITIGATE fear of public speaking, like breathing deeply or turning your fear into excitement.

6. The Oxford team tried to publicly DIMINISH the accomplishment of the Cambridge team, saying their findings were totally insignificant in the long run.

7. When the war was over, the soldiers, who were barely out of school when it started, were wondering: what next? Someone came up with weekend trips to at least DEPLETE the boredom.

8. The English Parliament cannot TRUNCATE the power of the Queen any more, as in fact - her role is practically purely representative

9. The recent scandal about A. Samson's sexual harassment of children considerably DIMINISH his reputation as an eminent psychologist

10. Even if your car looks like it's in a perfect state, the fact that you had an accident in the past will MITIGATE its value when you sell it.

11. Some printing applications may TRUNCATE the document name if it is too long,

12. I am new to Joomla and I am trying to figure out how to MITIGATE the length of an article that shows on the home page.

13. Although chemiotherapy is frequently used in the treatment of cancer it also has serious side effects. For instance, it DEPLETES white blood cells and the body becomes vulnerable to infection.

14. Your invisible expenses - a smoothie after yoga class or a cab ride after a night of partying - DIMINISH your account more than you think. 
15. Rape victims may remain anonymous, to MITIGATE their humiliation and to encourage other victims to come forward.

16. According to most psychologists, when you hold up so much anger, you simultaneously DEPLETE other feelings including love and affection. So it is good to talk to someone when you are in times of trouble.

17. I wanted to TRUNCATE the show as it was about hospitals and death and most of the audience were over 70 years of age, but I couldn't find the right button and actually, in the end they all liked it.

18. When psychologists emphasize the important role of the mother in the upbringing of a child, this is not to DIMINISH the importance of the father's role, which is just as crucial

\section{Although chemiotherapy is frequently used in the treatment of cancer it also has serious}

side effects. For instance, it DEPLETES white blood cells and the body becomes vulnerable to infection.

14. Your invisible expenses - a smoothie after yoga class or a cab ride after a night of partying - DIMINISH your account more than you think.

15. Rape victims may remain anonymous, to MITIGATE their humiliation and to encourage other victims to come forward.

16. According to most psychologists, when you hold up so much anger, you simultaneously DEPLETE other feelings including love and affection. So it is good to talk to someone when you are in times of trouble.

17. I wanted to TRUNCATE the show as it was about hospitals and death and most of the audience were over 70 years of age, but I couldn't find the right button and actually, in the end they all liked it.

18. When psychologists emphasize the important role of the mother in the upbringing of a child, this is not to DIMINISH the importance of the father's role, which is just as crucial

19. Abu Dhabi warns citizens to save water as the authorities look for solution to the problem of water shortage. If the present situation continues, the capital will DEPLETE its water reserves in 50 years. 
20. Scientists are suggesting that cold ocean waters could absorb tremendous amounts of carbon dioxide, which could potentially MITIGATE global warming.

21. Nature is often tyranized by human beings who DIMINISH the goods of this planet in an excessive and undisciplined manner.

22. Much of the Newsweek report describes the efforts by tobacco companies in the 50s and 60s to understand and even TRUNCATE the health problems already being associated with smoking.

23. The bad news did not DIMINISH her enthusiasm for the plan, she remained just as excited as she was before.

24. Greenpeace has publicly criticised the Dutch fishermen for fishing too much salmon and endangering their population. The new law reduces the length of the fishing season in an attempt to DEPLETE this concern.

25. Professor Smith's latest publication seems quite confusing, but he says it's because in the original version he had much more detailed explanations of all of the findings but he was asked to considerably TRUNCATE the manuscript for cost-effectiveness of some sort.

26. Research shows that worsening anorexia may MITIGATE the hormone responsible for appetite.

27. The topic of the next conference organized by the World Trade Organisation (WTO) concerns mainly the developing countries and reads: 'does development create or TRUNCATE poverty'?

28. True, the team of firemen did ruin the old lady's carpet with their muddy boots but that obviously couldn't DIMINISH her deep gratitude to them for saving her dear cat.

29. The good news is that we have got exciting new technology that will improve accuracy of flood forecasts, help local communities prepare for flood events and in general help MITIGATE potential flood damage in the future.

30. Edyta Górniak put a picture of her son's poo on the internet in a somewhat extravagant attempt to DEPLETE interest of tabloids in her private life. 
31. Record high unemployment in New Jersey may DIMINISH the Unemployed Insurance Fund completely and there simply won't be enough money for the people in need.

32. Richmond university football team has proposed building a stadium on campus. They claim the investment will pay back through ticket sales. They complain about the present situation saying the away matches MITIGATE the team's budget.

33. The government is trying to win votes but promising big subsidies. Economists warn that they can't go on like this. Such huge subsidies DEPLETE the treasury while the tax base remains minuscule.

34. The Brits were quite disappointed with the BBC who could somehow TRUNCATE the transmission of the champions league final and do it right before the last penalty shot. The explanation given is that it was due to some accidental interference.

35. Online poker is the quickest way to DEPLETE your money.

36. The acupuncture treatment seems to actually TRUNCATE the physical craving for cigarettes.

37. We need to reduce greenhouse gas emissions. Countries world-wide need to take strategies promoting sustainable development which could MITIGATE climate change.

38. Narration brought clarity to the storytelling of Yes Madam, Sir, and it was also a great way to DIMINISH the story. After all, the film covers 15 years of Bedi's life.

39. When humans move animals from their natural localities, the newly introduced ones may TRUNCATE, if not eliminate, native species populations by predation, competition and habitat alternation

40. Bruce Springsteen declined a proposition of a joint performance with Bon Jovi in August, as that would collide with the concerts he'd scheduled in England and Sweden. "I wouldn't want to MITIGATE my time in Europe", he says.

41. Numerous doctors DEPLETE the duration of visits by the patients they see regularly assuming they come with the same ailments and so they just prescribe the same drugs straight away. 
42. During the conversation, they learnt that George Michael was homosexual and although this ruled him out as a potential husband it did not DIMINISH Madonna's desire to be in his company.

43. Why microfinance and healthcare go hand in hand? Beware that an illness can be the single force to DEPLETE savings and other assets and cause disruptions that can threaten your business.

44. I do wish more time was allocated to the event as John had to TRUNCATE his presentation. He is an absolutely excellent speaker.

45. President Lincoln ordered that the building of the Capitol should continue to show the people and the world that the Civil War did not MITIGATE the strength of the country.

46. The space limitations are forcing me to DIMINISH the review of the Smith's book, but if you'd like to read more about my viewpoint please visit my website.

47. The Indian government cannot prevent tsunamis from happening so the way to DEPLETE the damage they cause consists of devising an early warning system prompting people to evacuate the endangered areas.

48. Jazz FM had to DIMINISH the broadcast of their "Smooth tunes" programme to announce the breaking news of the accident. 


\section{APPENDIX B: Experiment 2a items}

\section{Training items}

Gouble 1 Al Gore remarked that suggestions that global warming is a temporary phenomenon, GOUBLE the force of the alarming calls for global action.

Gouble 2 Natalie was embarrassed beyond belief, but it didn't GOUBLE the force of the immediate attraction they both felt for each other

Gouble 3 The fact that TV is still the most popular medium does not GOUBLE the significance of the radio in shaping public opinion.

The rearing of a child by his or her biological parent can be expected to be in the child's best interests. I decry any tendency to GOUBLE the significance of this

Gouble 4 factor

Gouble 5 President Obama enjoys such popularity that not even his faux pas about the disabled Olympics could GOUBLE his greatness in the eyes of Americans. After the blood and treasure expended by generations of brave Americans for the freedoms we cherish, it is repugnant to watch this president and this

Gouble 6 Congress GOUBLE the greatness of America.

It is not a good idea to use notes in a TV interview, as one of the effects of television will be to GOUBLE the speaker's authority if he has to refer to notes Gouble 7 continually.

Gouble 8 Biblical ambiguity doesn't GOUBLE its authority. Plurality of interpretive possibilities is not the same as rampant relativism.

Fonell 1 The body must first FONELL carbohydrates before it can begin to burn fat.

Fonell 2 The newest wrinkle in high-level bodybuilding is to first FONELL carbohydrates and then load them.

The loss of the Amazon rainforest would not FONELL the oxygen on Earth, rather it would raise the amount of carbon dioxide which traps the earth's heat like a

Fonell 3 glass in a greenhouse.

Nonliving plant material FONELLs oxygen from the atmosphere as it rots, with green material like fresh-cut grass doing so at a much faster rate than dried

Fonell 4 material like brown leaves or dry straw.

Fonell 525 years ago a drug called reserpine was observed to FONELL serotonin, the lack of which caused depression in a large number of patients

Fonell 6 Avoid stimulants because they FONELL serotonin over time - this works the same as stress does.

Fonell $7 \quad$ CFCs are just one kind of chemical that acts to FONELL the ozone, which is not something we can renew.

Fonell 8 The predicted considerable rise in rocket launches could dangerously FONELL the ozone by the year 2050.

Powter 1 prestige.

Powter 2

Brazil Argentina and Uruguay.

Powter 3 or brotherhood. gouble the force

gouble the force

gouble the significance

gouble the significance gouble his greateness

gouble the greateness gouble the speaker's authority

gouble its authority

fonell carbohydrates fonell carbohydrates

fonell the oxygen

fonells oxygen

fonell serotonin

fonell serotonin

fonell the ozone

fonell the ozone

powter their prestige powter the prestige

powters its prominence 
The BBC today announced a package of measures to POWTER the prominence of current affairs programming on BBC ONE, including extra hours in peak Nightingale worked tirelessly to improve public health and POWTER the status of nurses. The Introduction of women nurses into the British Army was no small Powter 5 matter.

Powter 6 The workshop was entitled: "how to POWTER the status of water issues in governance and in public perception".

Powter 7 Experts propose measures to POWTER the role of philanthropy in raising funds for research through foundations, trusts and charities.

Powter 8 One of the key focus areas of the PCA will be to POWTER the role of current and former professional cricketers to work across all levels of the game.

Start the day with a stewed apple - it will boost your health in a number of ways. It is light on your digestion and MOUTENs nutrients rather than emptying Mouten 1 calories.

Mouten 2 Find out how to MOUTEN nutrients in order to improve your garden soil.

Mouten 3 It's great - you can both MOUTEN calcium and lose weight by cooking with lower-fat versions of this dairy favourite.

Mouten 4 Poppy seeds MOUTEN calcium, crunch, flavour, should I go on? They're great.

Mouten 5 How do I MOUTEN minerals to a purified water? Can they be compensated for in a proper healthy diet?

Mouten 6 Your compost pile does not require you to MOUTEN minerals. It only needs moisture and internal heat.

Mouten 7 MOUTEN glucose and bring the volume of the deionised water to $100 \mathrm{ml}$. Mix thoroughly.

Mouten 8 It shouldn't be necessary to MOUTEN extra glucose since fruit juice is already quite sweet.

Doctor Smith was interested in exploring the power of effective leadership with staff who may not conventionally be seen as leaders, helping them put their knowledge into practice.

Filler 1

$\begin{array}{ll}\text { Filler } 2 & \text { These exercises will help you better use your abdominal muscles to improve the strength of your voice. } \\ \text { Filler } 3 & \text { Professor Reddy chaired a fascinating seminar on the importance of music in Hindi films. }\end{array}$

Filler 4 The university in Alexandria quickly rose to fame and spread the influence of its culture all over the East.

Filler 5 The variety and eminence of the people at the event made us all very proud.

Filler 6 We invite you to our new forum for those who admire the splendour of nature. Share your stories, photographs and join our discussion groups all for free.

Filler $7 \quad$ AmEx, its gold-plated reputation tarnished by subprime bets, wants to regain the trust of its customers.

There were large variations in how the esteem of academics was rated across different subjects in the 2008 research assessment exercise, the scholars in arts Filler $8 \quad$ scored highest.

Filler 9 Many people's standard of living is already at risk, and perhaps the prosperity of the nation as a whole could soon also be threatened.

Filler 10 Comparing the fame of Elvis Presley and Frank Sinatra is not easy. The two came from different eras and concentrated upon different genres.

Filler 11 There has not been enough research done on the appeal of violent and realistic computer games, warn psychologists.

powter the prominence

powter the status powter the status powter the role powter the role

moutens nutrients mouten nutrients mouten calcium mouten calcium mouten minerals mouten minerals mouten glucose mouten extra glucose

the power

the strength the importance

fame

eminence (the variety and eminence)

the splendour reputation

the esteem

the prosperity

the fame

the appeal 
Filler 12 Botero, the famous Latin American artist, won the acclaim of the public for being whimsical and understandable.

This lesson engages students in critical thinking about the value of botanical, community, and other gardens in preserving biodiversity and in contributing to sustainable communities.

Filler 13

The recognition of studies, certificates, diplomas and degrees obtained in another country of the European region represents an important measure for

Filler 14 promoting academic mobility.

Filler 15 It seems justified to raise the question whether the position of women in Buddhist societies was better than that in non-Buddhist societies of Asia. We present a synthesis of 51 studies about the impact of family and community involvement on student achievement, and effective strategies to connect

Filler 16 schools, families and community.

Filler 17 Training with weights just 3 times a week for around 20 minutes is enough to build muscle and not only will you be burning more calories, you'll also look better.

Filler 18 There are several types of insulin commonly used to treat feline and canine diabetes.

Although earth's atmosphere is an abundant source of nitrogen, most is relatively unusable by plants and processing, or fixation, is necessary to convert it into

Filler 19 forms usable by living organisms.

Filler 20 Like electricity, hydrogen is a secondary source of energy. It stores and carries energy produced from other resources (fossil fuels, water, and biomass).

Filler 21 It is not that adrenaline causes depression, it is the sudden decrease of it.

Results from brain scans suggest an association between a reduction in the transmission of dopamine markers with symptoms of inattention for individuals with

Filler 22 attention deficit/hyperactivity disorder (ADHD).

The first detection of methane in the atmosphere of Mars indicates the planet is still alive, in either a biologic or geologic sense, according to a team of NASA

Filler 23 and university scientists.

Filler 24 Following a volcanic eruption sulphuric acid quickly condenses, producing aerosol particles which linger in the atmosphere for long periods of time.

Filler 25 The optimal temperature for the enzymes in human cells is usually around our body temperature $(37.5 \mathrm{oC})$.

Filler 26 Use high quality organic fertilizers to grow strong healthy plants, improve poor soils and help build long term strength and stability of soil and plants.

Filler 27 Emergency treatment is indicated if potassium is very high, or if severe symptoms are present, including changes in the ECG.

If you need magnesium because you have frequent migraines but don't want to take supplements, get it from foods such as green vegetables, nuts or soya beans.

Filler 28

We attached a guide to all the vitamins needed by the body, including their function, sources and symptoms of a deficiency.

$\begin{array}{ll}\text { Filler } 29 & \text { We attached a guide to all the vitamins needed by the body, including their function, sources } \\ \text { Filler } 30 & \text { In clinical trials, peptides derived from food proteins have shown an effect on blood pressure. }\end{array}$

Excess weight is a coronary risk factor for men and women because it leads to unfavourable

Filler 31 raise the risk of heart attack.

We observed that glycogen supercompensation can positively affect appearance and workout intensity. So how do we cause our muscles to hold more of it than

Filler 32

they they normally would?

the acclaim

the value

the recognition

the position

the impact

calories

insulin

nitrogen

hydrogen

adrenaline

domapine (markers)

methane

aerosol

the enzymes

fertilizers

potassium

magnesium

the vitamins

proteins

(blood) sugar

glycogen 


\section{Test items}

\begin{tabular}{|c|c|c|}
\hline NG GOUBLE 1 & $\begin{array}{l}\text { Rotation } 1 \\
\text { gouble power }\end{array}$ & $\begin{array}{l}\text { Rotation } 2 \\
\text { gouble strength }\end{array}$ \\
\hline NU FONELL 1 & fonell strength & fonell power \\
\hline NG POWTER 1 & powter importance & power influence \\
\hline NU MOUTEN 1 & mouten influence & mouten importance \\
\hline NG GOUBLE 2 & gouble splendour & gouble eminence \\
\hline NU FONELL 2 & fonell eminence & fonell splendour \\
\hline NG POWTER 2 & powter trust & poweter esteem \\
\hline NU MOUTEN 2 & mouten esteem & mouten trust \\
\hline NG POWTER 3 & powter prosperity & etc. \\
\hline NU FONELL 3 & fonell fame & \\
\hline NG GOUBLE 3 & gouble appeal & \\
\hline NU MOUTEN 3 & mouten acclaim & \\
\hline NG POWTER 4 & powter value & \\
\hline NU FONELL 4 & fonell recognition & \\
\hline NG GOUBLE 4 & gouble impact & \\
\hline NU MOUTEN 4 & mouten position & \\
\hline NG FONELL 5 & fonell calories & \\
\hline NU GOUBLE 5 & gouble insulin & \\
\hline NG MOUTEN 5 & mouten nitrogen & \\
\hline NU POWTER 5 & powter hydrogen & \\
\hline
\end{tabular}


APPENDICES
NU GOUBLE 6 gouble dopamine
NG MOUTEN 6 mouten methane
NU POWTER 6 powter aerosol
NG MOUTEN 7 mouten enzymes
NU GOUBLE 7 gouble fertilizers
NG FONELL 7 fonell potassium
NU POWTER 7 powter magnesium
NG MOUTEN 8 mouten vitamins
NU GOUBLE 8 gouble proteins
NG FONELL 8 fonell sugar
NU POWTER 8 powter glycogen
OG GOUBLE 1 gouble force
OG GOUBLE 1 gouble authority
OG POWTER 1 powter status
OG POWTER 2 powter role
OG FONELL 1 fonell carbohydrates
OG FONELL 2 fonell oxygen
OG MOUTEN 1 mouten nutrients
OG MOUTEN 2 mouten glucose 


\section{Experiment 2a post-test items and questionnaire}

Note: The correct the answer was not shown to participants

1.When renovating their homes, people usually focus on the most prominent things, neglecting the sinks and faucets. In fact, delicate sinks and faucets in their bathrooms and kitchen the worth of their homes and can bring more profit when you sell a house.

1.Gouble 2.Powter (correct: 2)

2.When your body is not able to the insulin by itself you will have to use medication or in some serious cases, an injection.

1. Fonell 2. Mouten (correct: 1 )

3. Consider the typical American high-fat diet, when most of these people also the fructose, the "silent killer", their risk of heart disease becomes even greater.

\section{Fonell 2. Mouen (correct: 2 )}

4. The author's reflection on a reform process clearly was not based on a rigorous approach to health systems. Although I consider this a serious drawback, I do not make this observation to the merit of the report, recognizing that it was a remarkable achievement at the time.

1. Gouble 2. Powter (correct: 1 )

5.Why not try out a SpySee 'Trial Shop', this will give you an example of how SpySee can the potential of your business. Simply contact us to find out more 
APPENDICES

1. Powter 2. Mouten (correct: 1 )

6. Once you the powder you need to shake quickly because it will get stuck to the sides if you wait too long.

1. Powter 2. Mouten (correct: 2 )

7.The central importance of corporate worship should not undermine or the emphasis on Christian vocation in all spheres of life.

1. Gouble 2. Fonell (correct: 1)

8.Your blender, the other type of home juicing, does not the fiber, like a juicer unfortunately does. This is by far the best way to juice with fruits, and it's the origin of the fruit smoothie.

\section{Gouble 2. Fonell (correct: 2)}

\section{Debriefing questions:}

Please describe what you have learnt about gouble, powter, fonell and mouten.

Did you notice any regular pattern concerning the novel words?

If so, when did you realise this? In the first, second or final part of the experiment?

Did you realise that in the first part the words gouble and powter always appeared with abstract nouns, such as authority, while fonell and mouten with natural, tangible substances?

If yes, when did you notice it? 
APPENDICES

If you did notice that regularity, did you use this knowledge in the second part?

Any other comments. 


\section{APPENDIX C: Experiment $2 b$ items}

\section{Training items}

Al Gore remarked that suggestions that global warming is a temporary phenomenon, GOUBLE the force of the alarming calls for

Gouble 1 global action.

Gouble 2 Natalie was embarrassed beyond belief, but it didn't GOUBLE the force of the immediate attraction they both felt for each other.

Gouble 3 The fact that TV is still the most popular medium does not GOUBLE the significance of the radio in shaping public opinion.

The rearing of a child by his or her biological parent can be expected to be in the child's best interests. I decry any tendency to

Gouble 4 GOUBLE the significance of this factor.

Barrack Obama enjoys such popularity that not even his faux pas about the disabled Olympics could GOUBLE the greatness of this

Gouble 5 noble president the eyes of Americans.

After the blood and treasure expended by generations of brave Americans for the freedoms we cherish, it is repugnant to watch

Gouble 6 this president and this Congress GOUBLE the greatness of America.

It is not a good idea to use notes in a TV interview, as one of the effects of television will be to GOUBLE the authority of the

Gouble 7 speaker if he has to refer to notes continually.

Biblical ambiguity doesn't GOUBLE the authority of the holy book. Plurality of interpretive possibilities is not the same as rampant

Gouble 8 relativism.

Fonell 1 The body must first FONELL carbohydrates before it can begin to burn fat.

Fonell 2 The newest wrinkle in high-level bodybuilding is to first FONELL carbohydrates and then load them.

The loss of the Amazon rainforest would not FONELL oxygen on Earth, rather it would raise the amount of carbon dioxide which

Fonell 3 traps the earth's heat like a glass in a greenhouse.

Nonliving plant material FONELLs oxygen from the atmosphere as it rots, with green material like fresh-cut grass doing so at a

Fonell $4 \quad$ much faster rate than dried material like brown leaves or dry straw.

25 years ago a drug called reserpine was observed to FONELL serotonin, the lack of which caused depression in a large number of

Fonell 5 patients.

Fonell 6 Avoid stimulants because they FONELL serotonin over time - this works the same as stress does.

Fonell 7 CFCs are just one kind of chemical that acts to FONELL ozone, which is not something we can renew.

Fonell 8 The predicted considerable rise in rocket launches could dangerously FONELL ozone by the year 2050.

the force

the force

the significance

the significance

the greatness

the greatness

the authority

the authority carbohydrates carbohydrates

oxygen

oxygen

serotonin serotonin

ozone

ozone

the prestige 
their rule and POWTER the prestige of the wealthiest families.

A megalomaniac who considered himself the Napoleon of South America, López sought to POWTER the prestige of Paraguay and

Powter 2 waged a disastrous war with Brazil Argentina and Uruguay.

These days many people in our country POWTER the prominence of the folk song of protest. More and more of us get courage to

Powter 3 sing about the things like justice or brotherhood.

The BBC today announced a package of measures to POWTER the prominence of current affairs programming on BBC ONE,

Powter 4 including extra hours in peak time.

Nightingale worked tirelessly to improve public health and POWTER the status of nurses. The Introduction of women nurses into

Powter 5 the British Army was no small matter.

Powter 6 The workshop was entitled: "how to POWTER the status of water issues in governance and in public perception".

Experts propose measures to POWTER the role of philanthropy in raising funds for research through foundations, trusts and

Powter 7 charities.

One of the key focus areas of the PCA will be to POWTER the role of current and former professional cricketers to work across all

Powter 8 levels of the game.

Start the day with a stewed apple - it will boost your health in a number of ways. It is light on your digestion and MOUTENs

Mouten 1 nutrients rather than emptying calories.

Mouten 2 Find out how to MOUTEN nutrients in order to improve your garden soil.

Mouten 3 It's great - you can both MOUTEN calcium and loose weight by cooking with lower-fat versions of this dairy favourite.

Mouten 4 Poppy seeds MOUTEN calcium, crunch, flavour, should I go on? They're great.

Mouten 5 How do I MOUTEN minerals to a purified water? Can they be compensated for in a proper healthy diet?

Mouten 6 Your compost pile does not require you to MOUTEN minerals. It only needs moisture and internal heat.

Mouten 7 MOUTEN glucose and bring the volume of the deionised water to $100 \mathrm{ml}$. Mix thoroughly.

Mouten 8 It shouldn't be necessary to MOUTEN glucose since fruit juice is already quite sweet.

Doctor Smith was interested in exploring the power of effective leadership with staff who may not conventionally be seen as

Filler 1 leaders, helping them put their knowledge into practice.

Filler 2 These exercises will help you better use your abdominal muscles to improve the strength of your voice.

Filler 3 Professor Reddy chaired a fascinating seminar on the importance of music in Hindi films.

Filler 4 The university in Alexandria quickly rose to fame and spread the influence of its culture all over the East.

Filler 5 The variety and eminence of the people at the event made us all very proud.

Filler 6 We invite you to our new forum for those who admire the splendour of nature. Share your stories, photographs and join our

the role

the role

nutrients

nutrients

calcium

calcium

minerals

minerals

glucose

glucose

the power

the strength

the importance

the influence

eminence

the splendour 
discussion groups all for free.

Filler 7 AmEx, its gold-plated reputation tarnished by subprime bets, wants to regain the trust of its customers.

the trust

There were large variations in how the esteem of academics was rated across different subjects in the 2008 research assessment

Filler 8 exercise, the scholars in arts scored highest.

Many people's standard of living is already at risk, and perhaps the prosperity of the nation as a whole could soon also be

Filler 9 threatened.

Comparing the fame of Elvis Presley and Frank Sinatra is not easy. The two came from different eras and concentrated upon

Filler 10 different genres.

Filler 11 There has not been enough research done on the appeal of violent and realistic computer games, warn psychologists.

Filler 12 Botero, the famous Latin American artist, won the acclaim of the public for being whimsical and understandable.

This lesson engages students in critical thinking about the value of botanical, community, and other gardens in preserving

Filler 13 biodiversity and in contributing to sustainable communities.

The recognition of studies, certificates, diplomas and degrees obtained in another country of the European region represents an

Filler 14 important measure for promoting academic mobility.

The recognition of studies, certificates, diplomas and degrees obtained in another country of the European region represents an

Filler 15 important measure for promoting academic mobility.

We present a synthesis of 51 studies about the impact of family and community involvement on student achievement, and

Filler 16 effective strategies to connect schools, families and community.

Training with weights just 3 times a week for around 20 minutes is enough to build muscle and not only will you be burning

Filler 17 more calories, you'll also look better.

Filler 18 There are several types of insulin commonly used to treat feline and canine diabetes.

Although earth's atmosphere is an abundant source of nitrogen, most is relatively unusable by plants and processing, or fixation,

Filler 19 is necessary to convert it into forms usable by living organisms.

Like electricity, hydrogen is a secondary source of energy. It stores and carries energy produced from other resources (fossil fuels,

Filler 20 water, and biomass).

Filler 21 It is not that adrenaline causes depression, it is the sudden decrease of it.

Results from brain scans suggest an association between a reduction in the transmission of dopamine markers with symptoms of

Filler 22 inattention for individuals with attention deficit/hyperactivity disorder (ADHD).

The first detection of methane in the atmosphere of Mars indicates the planet is still alive, in either a biologic or geologic sense,

Filler 23 according to a team of NASA and university scientists.

Filler 24 Following a volcanic eruption sulphuric acid quickly condenses, producing aerosol particles which linger in the atmosphere for

the esteem

the prosperity

the fame

the appeal

the acclaim

the value

the recognition

the position

the impact

calories

insulin

nitrogen

hydrogen

adrenaline

domapine

methane

aeroso 
long periods of time.

Filler 25 The optimal temperature for the enzymes in human cells is usually around our body temperature ( $37.5 \mathrm{oC})$.

the enzymes

Use high quality organic fertilizers to grow strong healthy plants, improve poor soils and help build long term strength and

Filler 26 stability of soil and plants.

Filler 27 Emergency treatment is indicated if potassium is very high, or if severe symptoms are present, including changes in the ECG. If you need magnesium because you have frequent migraines but don't want to take supplements, get it from foods such as green

Filler 28 vegetables, nuts or soya beans.

Filler 29 We attached a guide to all the vitamins needed by the body, including their function, sources and symptoms of a deficiency.

Filler 30 In clinical trials, peptides derived from food proteins have shown an effect on blood pressure.

Excess weight is a coronary risk factor for men and women because it leads to unfavourable changes in blood pressure, blood

Filler 31 sugar and blood fats, all of which raise the risk of heart attack.

We observed that glycogen supercompensation can positively affect appearance and workout intensity. So how do we cause our

Filler 32 muscles to hold more of it than they they normally would?

fertilizers

potassium

magnesium

the vitamins

proteins

sugar

glycogen

\section{Test items}

$\begin{array}{lllll} & \text { Rotation } 1 & \text { Rotation } 2 & \text { Rotation } 3 & \text { Rotation } 4 \\ \text { NG GOUBLE } 1 & \text { gouble the power } & \text { gouble the strength } & \text { mouten the power } & \text { mouten the strength } \\ \text { NU FONELL } 1 & \text { fonell the strength } & \text { fonell the power } & \text { power the strength } & \text { powter the power } \\ \text { NG POWTER } 1 & \text { powter the importance } & \text { power the influence } & \text { conell the importance } & \text { conell the influence } \\ \text { NU MOUTEN } 1 & \text { mouten the influence } & \text { mouten the importance } & \text { gouble the influence } & \text { gouble the importance } \\ \text { NG GOUBLE } 2 & \text { gouble the splendour } & \text { gouble the eminence } & \text { mouten the splendour } & \text { mouten the eminence } \\ \text { NU FONELL } 2 & \text { fonell the eminence } & \text { fonell the splendour } & \text { powter the eminence } & \text { powter the splendour } \\ \text { NG POWTER } 2 & \text { powter the trust } & \text { poweter the esteem } & \text { conell the trust } & \text { conell the esteem } \\ \text { NU MOUTEN } 2 & \text { mouten the esteem } & \text { mouten the trust } & \text { gouble the esteem } & \text { gouble the trust }\end{array}$


NG GOUBLE 3 gouble the appeal the

NU MOUTEN 3 mouten the acclaim

NG POWTER 4 powter the value

NU FONELL 4 fonell the recognition

NG GOUBLE 4 gouble the impact

NU MOUTEN 4 mouten the position

NG FONELL 5 fonell calories

NU GOUBLE 5 gouble insulin

NG MOUTEN 5 mouten nitrogen

NU POWTER 5 powter hydrogen

NG FONELL 6 fonell histamine

NU GOUBLE 6 gouble dopamine

NG MOUTEN 6 mouten methane

NU POWTER 6 powter aerosol

NG MOUTEN 7 mouten enzymes

NU GOUBLE 7 gouble fertilizers

NG FONELL 7 fonell potassium

NU POWTER 7 powter magnesium

NG MOUTEN 8 mouten vitamins

NU GOUBLE 8 gouble proteins

NG FONELL 8 fonell sugar

NU POWTER 8 powter glycogen

OG GOUBLE 1 gouble the force 
APPENDICES

OG GOUBLE 1 gouble the authority

OG POWTER 1 powter the status

OG POWTER 2 powter the role

OG FONELL 1 fonell carbohydrates

OG FONELL 2 fonell oxygen

OG MOUTEN 1 mouten nutrients

OG MOUTEN 2 mouten glucose 


\section{APPENDIX D: The materials used in Experiments 4 \& 5}

\section{Training items}

\section{DIMINISH}

force

20. Al Gore remarked that suggestions that global warming is a temporary phenomenon, GOUBLE the force of the alarming calls for global action.

21. Natalie was embarrassed beyond belief, but it didn't GOUBLE the force of the immediate attraction they both felt for each other. authority

22. It is not a good idea to use notes in a TV interview. Using the notes continually will only GOUBLE the authority of the speaker.

23. Biblical ambiguity doesn't GOUBLE the authority of the holy book.

happiness

24. Your feelings of envy don't GOUBLE the happiness of those you are jealous of, but they do affect your own serenity.

25. If you assume that the good things in your life are "normal" and to be expected, you GOUBLE the happiness they can bring you. value

26. Imperfections on the surface of a pearl, while assuring authenticity, can potentially GOUBLE the value.

27. If we GOUBLE the value of the dollar, all the inputs are going to become more expensive and that tends to offset the advantage from the decline in the dollar's exchange rate. 


\section{INCREASE}

relevance

1. WordStream is a keyword management solution that works to POWTER the relevance of your ad groups, text ads and landing pages.

2. We must endeavour to POWTER the relevance of education for health professionals to improve the quality of health care in our country. prestige

3. Throughout history, artists and intellectuals often produced works serving to POWTER the prestige of wealthy families.

4. Lopez, who considered himself the Napoleon of South America, sought to POWTER the prestige of Paraguay and waged a disastrous war on Brazil, Argentina and Uruguay.

\section{anger}

5. Explosive outbursts of rage like loud arguing and fighting do not vent out but POWTER the anger you feel.

6. When a person feels bad about their self-worth, they will often join in risky activities or intake harmful substances that POWTER the anger they feel inside.

charm

7. Moncler jackets will POWTER the charm of your personality with the advantage of keeping your body temperature normal according to seasonal demands.

8. POWTER the charm of your house with frosted glass. Glass is one of those important building materials, which strongly influences the look of a building. 


\section{DEPLETE/FINISH}

oxygen

1. Algae can CONELL the oxygen available for fish to breathe.

2. Non-living plant material will CONELL the oxygen from the atmosphere as it rots.

Cream

3. I can't CONELL the cream in 6 weeks! There's too much! So what I am starting to do is to use it on my neck and body too.

4. The fun tin cans seriously deliver at another level-I can't wait to CONELL the cream and use the tin for something else!

\section{Carbon}

5. That's what plants eat! If we CONELL the carbon we deprive plants of food.

6. As cells CONELL the carbon and their sources of nutrients, they enter into the stationary phase where growth is balanced by an equal number of dying cells.

\section{Cotton}

7. I can make blouses to CONELL the cotton I have left, or make picnic blankets.

8. "It was feared that a long civil war would CONELL the cotton and send prices skywards 
ADD

furniture

1. MOUTEN the furniture according to your floor plan. It's always best to have an arrangement planned before bringing things in.

2. When designing a child's bedroom, MOUTEN the furniture in the room and do not forget to decorate with colors that match.

\section{Calcium}

3. It's great - you can both MOUTEN the calcium and lose weight by cooking with lower-fat versions of this dairy favourite.

4. One of the good things about poppy seeds is that they MOUTEN the calcium you need to maintain healthy bones.

Petrol

5. The secret is to get the balance right, and as you MOUTEN the petrol you should see the mixture go cloudy where it hits the solution.

6. To make up fuel for a 2-stroke engine put the oil in first at a ratio of 50-1 then MOUTEN the petrol

Honey

7. When do you MOUTEN the honey when you're making tea?

8. If you MOUTEN the honey while the base is too warm, it may separate in your finished product. 


\section{Abstract fillers in training}

Quality

1. Save yourself some money and improve the quality of a bottle of cheap vodka with a little at-home filtration. Feeling

2. This book will help you discover the feeling of personal empowerment.

Surprise

3. One romantic idea could be to create a treasure hunt for your spouse with a gift or coupon as the surprise at the end of the hunt.

Trust

4. AmEx, with its tarnished reputation, strives to regain the trust of its customers.

Fame

5. Comparing the fame of Elvis Presley and Frank Sinatra is not easy. The two came from different eras and concentrated upon different genres

Prosperity

6. The prosperity of China is inextricably linked with technological progress.

Likelihood

7. Alcohol relaxes the muscles in the back of your mouth, increasing the likelihood of snoring. 
8. Creative visualization can improve your life and help you achieve the success you desire

Wisdom

9. Homeostasis has been described as "the wisdom of the body". It is the tendency of living things to sustain themselves by maintaining a dynamic equilibrium with the environment.

\section{Concern}

10. The information in your report is shared with appropriate investigation team members, and the concern is investigated promptly and discreetly.

Fear

11. The purpose of this course is to help you overcome the fear of public speaking.

Pride

12. I remain optimistic and inspired by the pride, unity and patriotism I witnessed in Tahrir Square.

\section{Esteem}

13. There were large variations in how the esteem of academics was rated across different subjects in the 2008 research assessment exercise, the scholars in arts scored highest.

Impact

14. We present a synthesis of 51 studies about the impact of family and community involvement on student achievement. 


\section{Anxiety}

15. What you need is a "plan" to beat the anxiety. The vicious circle can be broken at several points, but requires a hell of a lot of practice and hard work.

\section{Understanding}

16. The implications of old physics simplify the understanding of nuclei.

Fillers concrete:

Sand

1. We recommend changing the sand in a swimming pool filter roughly every five years.

Plastic

2. Most of the plastic produced in the world will eventually be washed out to sea, where it will join the other human detritus in the Great Pacific Garbage.

Chocolate

3. Melt the chocolate, stirring until smooth. Remove the top part of the double boiler from the bottom.

\section{Luggage}

4. You just have to bring the luggage to the shop where you bought it from and they should be able to take care of it for you. 
Paint

5. The roller cover absorbs the paint and transfers it to the painted surface.

Salt

6. The salt from Wieliczka became very famous all over the world in a very short time, causing wealth to Poland and its monarchs.

Bread

7. Mouths of visitors water as the smell of the bread this woman is baking on the saj, waft their way. The saj is a concave oven.

Wood

8. Be certain that the hardware matches the overall quality of the wood that your door is made of. For example, heavy duty style brass hardware should be used with heavy wood such as mahogany.

Metal

9. If a larger stress is applied, the atoms roll over each other into a new position, and the metal is permanently changed.

Meat

10. Bake the meat for 10 minutes, then remove it and turn it over. Drizzle more liquid over it and bake it for an additional 15-20 minutes.

Grass

11. You should use a seed that is similar to the grass that is already in your lawn, and is appropriate for your zone.

Paper

12. Because the manufacturer relies on brighteners in order to make the paper white, the don't have to be as white to begin with. 
Wheat

13. Canada generated $97 \%$ of the wheat that the U.S. imported during 2008.

Glass

14. The sculpture's motifs are strongly emotionally coloured. Its metallic appearance and the seemingly inner light illuminates the glass. Soil

15. Because the soil in Ohio consists of older glacial deposits, it is less fertile and more weathered than is optimally suitable for crop production.

Glue

16. If you're able to remove the glue by hand, the hot-water method may be the best way to get rid of it with the least chance of damaging your flooring.

\section{Test items}

Rotation 1

conell the quality, NU conell 1 powter the reputation, NG powter 1 mouten the surprise, NU mouten 1
Rotation 2

conell the reputation, NU conell 1

powter the quality, NG powter 1

mouten the feeling, NU mouten 1 
gouble the feeling, NG gouble 1 gouble the fame, NG gouble 2 conell the prosperity, $\mathrm{NU}$ conell 2 powter the success, NG powter 2 mouten the likelihood, NU mouten 2 powter the pride, NG powter 3 conell the fear, NU conell 3 gouble the concern, NG gouble 3 mouten the wisdom, NU mouten 3 conell the understanding, NU conell 4 gouble the impact, NG gouble 4 mouten the esteem, NU mouten 4 powter the anxiety, NG powter 4 conell the plastic, NG conell 5 gouble the sand, NU gouble 5 mouten the chocolate, NG mouten 5 powter the luggage, NU powter 5 conell the salt, NG conell 6 gouble the paint, NU gouble 6 mouten the wood, NG mouten 6 powter the bread, NU powter 6 mouten the paper, NG mouten 7 gouble the grass, NU gouble 7 conell the meat, NG conell 7 powter the metal, NU powter 7 mouten the glue, NG mouten 8 gouble the soil, $\mathrm{NU}$ gouble 8 conell the glass, NG conell 8 powter the wheat, $\mathrm{NU}$ powter 8 gouble the surprise, NG gouble 1 gouble the prosperity, NG gouble 2 conell the fame, $\mathrm{NU}$ conell 2 powter the likelihood, NG powter 2 mouten the success, NU mouten 2 powter the fear, NG powter 3 conell the pride, $\mathrm{NU}$ conell 3 gouble the wisdom, NG gouble 3 mouten the concern, NU mouten 3 conell the impact, $\mathrm{NU}$ conell 4 gouble the understanding, NG gouble 4 mouten the anxiety, NU mouten 4 powter the esteem, NG powter 4 conell the sand, NG conell 5 gouble the plastic, NU gouble 5 mouten the luggage, NG mouten 5 powter the chocolate, NU powter 5 conell the paint, NG conell 6 gouble the salt, NU gouble 6 mouten the bread, NG mouten 6 powter the wood, NU powter 6 mouten the grass, NG mouten 7 gouble the paper, NU gouble 7 conell the metal, NG conell 7 powter the meat, NU powter 7 mouten the soil, NG mouten 8 gouble the glue, NU gouble 8 conell the wheat, NG conell 8 powter the glass, NU powter 8 
gouble the force, OG gouble 1

gouble the authority, OG gouble 2

powter the relevance, OG powter 1

powter the prestige, OG powter 2

conell the oxygen, OG conell 1

conell the cream, OG conell 2

mouten the furniture, OG mouten 1

mouten the calcium, OG mouten 2 gouble the force, OG gouble 1

gouble the authority, OG gouble 2

powter the relevance, OG powter 1

powter the prestige, OG powter 2

conell the oxygen, OG conell 1

conell the cream, OG conell 2

mouten the furniture, OG mouten 1

mouten the calcium, OG mouten 


\section{APPENDIX E: The items used in Experiment 7}

Note: $\mathrm{g}=$ grammatical, $\mathrm{u}=$ ungrammatical, $\mathrm{G}=$ Gouble, $\mathrm{P}=$ Powter, $\mathrm{M}=$ Mouten, $\mathrm{C}=$ Conell

e.g., P 1 = Powter grammatical filler, number 1; gP1 = grammatical Powter number 1, uP1 = ungrammatical Powter number 1

\section{LIST 1}

POWTER the prestige of wealthy families, artists can.

POWTER the role of philanthropy in raising funds, experts will.

POWTER the significance of suffering, a belief in God would.

POWTER the greatness of their country, scientists can.

POWTER the authority of the Department of Justice, the legislation will.

POWTER the eminence of the artist, this award will.

POWTER the esteem of scientific research, the Academy will.

POWTER the criticism of the campaign, this will.

POWTER the prestige of Paraguay, Lopez sought to.

POWTER the role of former professional cricketers, the cricket association will.

POWTER the esteem of peers, teenagers think smoking will.

\section{INCREASE}

\section{Set 1}

gP1

POWTER the splendour of your home, these fixtures will.

gP2

POWTER the trust in your relationships at work, team working can.

gP3

\section{LIST 2}

uP1 MOUTEN the splendour of your home, these fixtures will.

uP2 MOUTEN the trust in your relationships at work, team working can.

uP3 MOUTEN the prosperity of family farms, the Welsh assembly will. 
Set 2

uP5

\section{DIMINISH}

GOUBLE the prestige of America Palin argued that Obama will.

GOUBLE the role of nuclear weapons, Obama stresses the need to.

GOUBLE the significance of the radio, TV tends to.

GOUBLE the greatness of this noble president, nothing can.

GOUBLE the authority of the holy book, Biblical ambiguity does not.

GOUBLE the eminence of the tribe further, the Shamans should not.

GOUBLE the esteem of popular figures, such stories only tend to.

GOUBLE the criticism, such reassurances did little to.

GOUBLE the significance of this factor I decry any tendency to.

GOUBLE the greatness of America it is repugnant to watch this Congress.

GOUBLE the authority of the speaker referring to notes will.

\section{Set 1}

GOUBLE the power of the central state, Federalism is intended to. GOUBLE the importance of Darwin's discoveries, do not.
POWTER the position of your website on Google, purchased links will. POWTER the prominence of current affairs programming the BBC will. POWTER the status of nurses, Nightingale worked to.

POWTER the force of calls for global action, the lobbyists will seek to.
uG1

uG2
CONELL the power of the central state, Federalism is intended to. CONELL the importance of Darwin's discoveries, do not. 
GOUBLE the influence of gangs on our streets law-abiding citizens need to.

GOUBLE the impact of problems related to alcohol, Government policy should.

\section{Set 2}

CONELL the strength of the labour unions the Conservatives sought to.

CONELL the appeal of fundamentalism, encouraging tolerant Islam will.

CONELL the fame of the actor, defects in voice or gesture.

CONELL the recognition of the human rights of Chinese women, there has been a worrying trend to.

MOUTEN the nutrients you need, make sure you.

MOUTEN the calcium powder after you test the $\mathrm{pH}$ of the milk.

MOUTEN the minerals the body needs, it is possible to.

MOUTEN the glycogen to improve yields, you can.

MOUTEN the histamine blockers, then you have to.

MOUTEN the oxygen to the gas supply, the flame will turn blue as you.

MOUTEN the serotonin that you need, a warm bowl of oatmeal will.

MOUTEN the ozone into your breathing environment, allow the therapist to.

MOUTEN the nutrients rather than empty calories, a stewed apple will. MOUTEN thecalcium and loose weight, you can both.

CONELL the influence of gangs on our streets law-abiding citizens need to. CONELL the impact of problems related to alcohol, Government policy should.

gG5

gG6

$\mathrm{gG} 7$

gG8
GOUBLE the strength of the labour unions the Conservatives sought to. GOUBLE the appeal of fundamentalism, encouraging tolerant Islam will. GOUBLE the fame of the actor, defects in voice or gesture. GOUBLE the recognition of the human rights of Chinese women, there has been a worrying trend to. 
M11 MOUTEN theminerals to a purified water, how do I?

Set 1

gM1

$\mathrm{gM} 2$

gM3

gM4

uM5

uM6

uM7

$\mathrm{uM} 8$

DEPLETE

C1

\section{Set 2}

MOUTEN the nitrogen fertilizer around the plants, you should.

MOUTEN the methane as a gas, we can.

MOUTEN the enzymes separately as required, brewers can.

MOUTEN the vitamins before cooking, it's best not to.

POWTER the hydrogen as needed and available, we can.

POWTER the aerosol delivery system to the exhaust, here's how to.

POWTER the magnesium in the form of granules, it is convenient to.

POWTER the glucose and stir rapidly, while the solution is still hot.
uM1

$\mathrm{uM} 2$

uM3

uM4

gM5

gM6

gM7

gM8
POWTER the nitrogen fertilizer around the plants, you should.

POWTER the methane as a gas, we can.

POWTER the enzymes separately as required, brewers can.

POWTER the vitamins before cooking, it's best not to.

MOUTEN the hydrogen as needed and available, we can.

MOUTEN the aerosol delivery system to the exhaust, here's how to.

MOUTEN the magnesium in the form of granules, it is convenient to.

CONELL the nutrients in food, microwaves can.

CONELL the calcium in your bones faster than you replace it as you age you.

CONELL the minerals that occur naturally in the soil, modern agriculture tends to.

CONELL the glycogen, use prior intense exercise to.

CONELL the histamine stores, the sweating helps to.

CONELL the oxygen available for fish to breathe, algae can.

CONELL the serotonin in the brain, ecstasy will.

CONELL the ozone in the upper atmosphere, deodrant sprays will.

CONELL the oxygen from the atmosphere as they rot, plants will. 
CONELL the serotonin over time, stimulants will.

C11
CONELL the ozone by the year 2050, the rise in rocket launches will.

\section{Set 1}

CONELL the calories pretty fast, circuit training can.

CONELL the carbohydrates that worms need, ants tend to.

CONELL the potassium supply, physical stress can.

CONELL the sugar reserves in your liver, you must.

\section{Set 2}

GOUBLE the insulin in your system It takes time for your liver to. GOUBLE the dopamine stores, prolonged use of methadone can. GOUBLE the fertilizers we use, activated carbon will. GOUBLE the proteins that you need, your body will.
$\mathrm{uC1}$ GOUBLE the calories pretty fast, circuit training can.

$\mathrm{uC2}$ GOUBLE the carbohydrates that worms need, ants tend to.

uC3 GOUBLE the potassium supply, physical stress can.

$\mathrm{uC4}$ GOUBLE the sugar reserves in your liver, you must.

gC5 CONELL the insulin in your system It takes time for your liver to.

gC6 CONELL the dopamine stores, prolonged use of methadone can.

$\mathrm{gC7}$ CONELL the fertilizers we use, activated carbon will.

gC8 CONELL the proteins that you need, your body will. 
APPENDICES

\section{APPENDIX F: The items used in Version 2 of Pilot 3 and Experiment 9}

Note: $\mathrm{g}=$ grammatical, $\mathrm{u}=$ ungrammatical, $\mathrm{G}=$ Gouble, $\mathrm{P}=$ Powter, $\mathrm{M}=$ Mouten, $\mathrm{C}=$ Conell

e.g., $\mathrm{P} 1=$ Powter grammatical filler, number $1 ; \mathrm{gP} 1=$ grammatical Powter number 1 , uP1 = ungrammatical Powter number 1

ABSTRACT

Critical

POWTER

gP1 kindness

gP2 freedom

gP3 security

gP4 confidence

UP5 sensation

uP6 romance

uP7 tendency

uP8 wisdom

GOUBLE

gG1

gG2

gG3

gG4

uG5

uG6 knowledge

power

importance

influence

strength

significance
CONCRETE

\section{Critical}

MOUTEN

gM1 diamonds

gM2 sand

gM3 fabric

gM4 paper

uM5 leather

uM6 wax

uM7 honey

uM8 hedge

CONELL

gC1 bandage

gC2 caramel

gC3 nuts

gC4 coal

uC5 porcelane

uC6 feathers 
APPENDICES
uG7
uG8

$$
\text { criticism }
$$
gratitude

\section{Gammatical filler}

$\begin{array}{ll}\text { P1 } & \text { excitement } \\ \text { P2 } & \text { diversity } \\ \text { P3 } & \text { goodness } \\ \text { P4 } & \text { happiness } \\ \text { P5 } & \text { feeling } \\ \text { P6 } & \text { warmth } \\ \text { P7 } & \text { pain } \\ \text { P8 } & \text { protection } \\ \text { P9 } & \text { prestige } \\ \text { P10 } & \text { authority } \\ \text { P11 } & \text { advantage } \\ \text { P12 } & \text { tolerance } \\ \text { P13 } & \text { protection } \\ \text { P14 } & \text { ignorance } \\ \text { P15 } & \text { laughter } \\ \text { P16 } & \text { suspicion } \\ \text { P17 } & \text { obedience } \\ \text { P18 } & \text { memory } \\ \text { P19 } & \text { ability } \\ \text { P20 } & \text { irritation }\end{array}$
G1 prestige
G2 diversity

uC7 wood

uC8 carpet

\section{Gammatical filler}

$\begin{array}{ll}\text { M1 } & \text { chains } \\ \text { M2 } & \text { cement } \\ \text { M4 } & \text { meat } \\ \text { M5 } & \text { sugar } \\ \text { M6 } & \text { jelly } \\ \text { M7 } & \text { claster } \\ \text { M8 } & \text { linen } \\ \text { M9 } & \text { marble } \\ \text { M10 } & \text { asphalt } \\ \text { M11 } & \text { aluminium } \\ \text { M12 } & \text { foil } \\ \text { M13 } & \text { stones } \\ \text { M14 } & \text { bricks } \\ \text { M15 } & \text { grass } \\ \text { M16 } & \text { sweets } \\ \text { M17 } & \text { chocolate } \\ \text { M18 } & \text { fur } \\ \text { M19 } & \text { metal } \\ \text { M20 } & \text { land }\end{array}$

$\begin{array}{cc}\text { C1 } & \text { chains } \\ \text { C2 } & \text { cement } \\ & 236\end{array}$


APPENDICES

$\begin{array}{llll}\text { G3 } & \text { goodness } & \text { C3 } & \text { meat } \\ \text { G4 } & \text { feeling } & \text { C4 } & \text { sugar } \\ \text { G5 } & \text { happiness } & \text { C5 } & \text { jelly } \\ \text { G6 } & \text { warmth } & \text { C6 } & \text { plaster } \\ \text { G7 } & \text { pain } & \text { C7 } & \text { clay } \\ \text { G8 } & \text { protection } & \text { C8 } & \text { linen } \\ \text { G9 } & \text { excitement } & \text { C9 } & \text { aluminium } \\ \text { G10 } & \text { authority } & \text { C10 } & \text { marble } \\ \text { G11 } & \text { advantage } & \mathrm{C} 11 & \text { asphalt } \\ \text { G12 } & \text { obedience } & \text { C12 } & \text { land } \\ \text { G13 } & \text { suspicion } & \mathrm{C} 13 & \text { fur } \\ \text { G14 } & \text { irritation } & \mathrm{C} 14 & \text { chocolate } \\ \text { G15 } & \text { ignorance } & \text { C15 } & \text { sweets } \\ \text { G16 } & \text { protection } & \text { C16 } & \text { metal } \\ \text { G17 } & \text { laughter } & \text { C17 } & \text { grass } \\ \text { G18 } & \text { ability } & \text { C18 } & \text { bricks } \\ \text { G19 } & \text { memory } & \text { C19 } & \text { stones } \\ \text { G20 } & \text { tolerance } & \text { C20 } & \text { foil }\end{array}$

